Historic, archived document

Do not assume content reflects current scientific knowledge, policies, or practices. 


\section{United States Department of Agriculture}

Forest

Service

North Central

Forest Experiment

Station

Resource

Bulletin NC-108

iins

\section{Indiana Forest Statistics, 1986}

W. Brad Smith and Mark F. Golitz

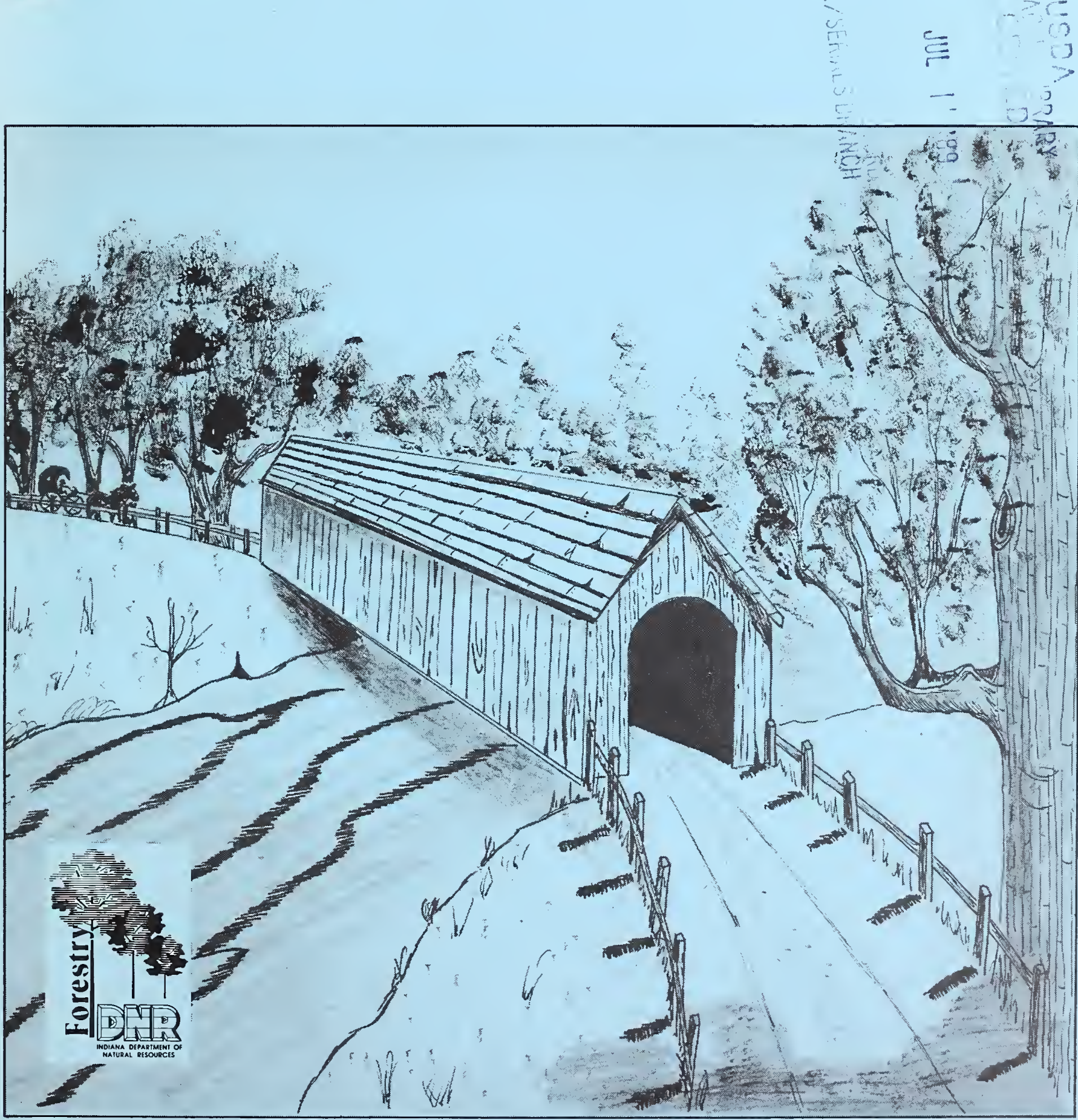


Forest Inventory and Analysis local artist series No. 1 "Lin Montgomery, Brownstown, Indiana" 
This report includes the most commonly used Forest Inventory and Analysis statistics. However, additional forest resource data can be provided to interested users. Persons requesting additional information that can be provided from the raw inventory data are expected to pay the retrieval costs. These costs range from less than $\$ 100$ for a relatively simple request to $\$ 2,000$ for a complex retrieval involving the services of a Forest Inventory and Analysis computer programmer. Requests will be filled so as to minimize the impact on the Forest Inventory and Analysis Work Unit.

Requests for unpublished information may be directed to:

Project Leader

Forest Inventory and Analysis Project

North Central Forest Experiment Station

1992 Folwell Avenue

St. Paul, Minnesota 55108

Phone: (612) 649-5140

Area served: Illinois, Indiana, Iowa, Kansas, Michigan, Minnesota, Missouri, Nebraska, North Dakota, South Dakota, Wisconsin.

Requests for unpublished information from the Indiana inventory may also be directed to:

State Forester

Indiana Department of Natural Resources

Division of Forestry

613 State Office Building

Indianapolis, Indiana 46204 


\section{FOREWORD}

Forest Inventory and Analysis (FIA) is a continuing endeavor as mandated by the Renewable Forest and Rangeland Resources Planning Act of 1974. Prior inventories were mandated by the McSweeney-McNary Forest Research Act of 1928. The objective of FIA is to periodically inventory the Nation's forest land to determine its extent, condition, and volume of timber, growth, and depletions. Up-to-date resource information is essential to frame intelligent forest policies and programs. USDA Forest Service regional experiment stations are responsible for conducting these inventories and publishing summary reports for individual States. The North Central Forest Experiment Station is responsible for forest resource evaluation in Illinois, Indiana, Iowa, Kansas, Michigan, Minnesota, Missouri, Nebraska, North Dakota, South Dakota, and Wisconsin.

Fieldwork for the Indiana Statewide forest inventory was begun in July 1985 and completed in December 1986. Reports on the two previous inventories of Indiana's timber resource are dated 1950 and 1967.

More accurate survey information was obtained during the 1986 survey than otherwise would have been feasible because of intensified field sampling. Such sampling was made possible by additional funding provided by the Indiana State Legislature through the Division of Forestry, Indiana Department of Natural Resources. The Department also surveyed primary wood-using plants in the State. Data from this survey were used to help estimate the quantity of timber products harvested in the State. Indiana Department of Natural Resources personnel have also assisted in training field personnel, analyzing information obtained from the survey, and preparing this report.

Aerial photos used in the Indiana Forest Inventory were furnished by the Hoosier National Forest and the USDA Agricultural Stabilization and Conservation Service. 


\section{CONTENTS}

Page

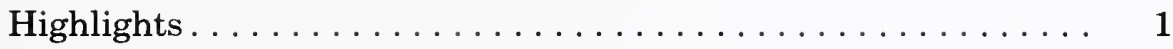

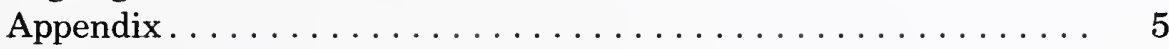

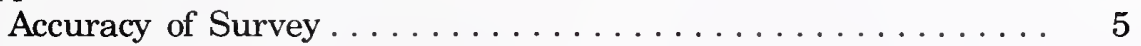

Survey Procedures ...................... 5

Comparing Indiana's Third Inventory

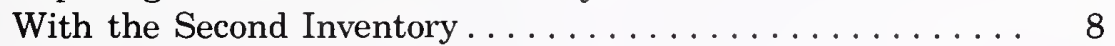

Log Grade......................... 9

Metric Equivalents of Units Used in This Report . . . . . . . . 13

Tree Species Groups in Indiana . . . . . . . . . . . . . . . . 14

Definition of Terms ...................... 15

Tables ... . . . . . . . . . . . . . . . . . 23 



\title{
INDIANA FOREST STATISTICS, 1986
}

\author{
W. Brad Smith, \\ and Mark F. Golitz
}

\section{HIGHLIGHTS}

\section{Forest Area}

- Forest land was 4.4 million acres (19.3 percent of the State's total land area) in 1986.

- Timberland (formerly called commercial forest land) increased 10 percent between 1967 and 1986, from $3,895,800$ to $4,295,800$ acres. The largest increase occurred in the Upland Flats Unit primarily due to reversion of wooded pasture and marginal farmland.

- Reserved timberland totaled 143,400 acres in 1986, 3.2 percent of the State's forest land. There were only 38,500 acres of reserved timberland in the State in 1967.

- Timberland represented 18.7 percent of the State's total land area (fig. 1).

- Perry County contained the largest area of timberland in 1986 (152,500 acres), as it did in 1967 (142,800 acres).

- Farmers and miscellaneous private individuals owned $3,335,400$ acres -78 percent of the timberland. A new definition of farm ownership shifted much of what was previously in the farmer category to the miscellaneous private category (fig. 2).

- The predominant forest type, oak-hickory, accounted for 33 percent $(1,436,700$ acres $)$ of the total timberland in 1986, down from 61 percent of the total in 1966.

The area of the oak-hickory type is declining for several reasons: Many mature oak-hickory stands where no cutting has taken place are succeeding into the maple-beech and cherry-ashpoplar types. In many oak-hickory stands where

W. BRAD SMITH is a Mensurationist with the North Central Forest Experiment Station, St. Paul, Minnesota; and MARK F. GOLITZ is a Forest Resource Planner, Indiana Department of Natural Resources, Division of Forestry, 613 State Office Building, Indianapolis, Indiana 46204. cutting has taken place, soft maple and yellowpoplar are the predominant species to regenerate, producing a different forest type.

- The area of sawtimber stands increased 36 percent between inventories, while the area of poletimber stands decreased 22 percent (fig. 3).

\section{Volume}

- The volume of growing stock on timberland was 5.2 billion cubic feet in 1986, up 43 percent from $1967^{1}$.

- Sawtimber volume on timberland totaled 19.2 billion board feet ${ }^{2}$ in 1986, up 54 percent from $1967^{3}$.

- Hardwoods accounted for more than 96 percent of both the growing-stock volume ( 5.0 billion cubic feet) and the sawtimber volume (18.6 billion board feet) on the State's timberland.

- The oak species account for 36 percent (6.9 billion board feet) of the total sawtimber volume.

- Growing-stock volume of the oaks and hickories increased 12 percent between 1967 and 1986, while all other species increased 77 percent over the same period. The harvesting pressure on oak and hickory is apparent, as these species' total share of inventory declined from 53 to 41 percent of growing stock between 1967 and 1986. This decrease in oak as a percentage of the total volume is a major factor in the decline in the area of the oakhickory forest type (fig. 4).

- Yellow-poplar growing-stock volume increased 130 percent, from 188 million cubic feet in 1967 to 433 million cubic feet in 1986. The growing-stock volume of yellow-poplar was 8 percent of the total in 1986, compared to 5 percent of the total in 1967.

\footnotetext{
${ }^{1}$ Because of changes in volume equations, 1967 growing-stock volumes have been adjusted from those published after the 1967 survey to conform to 1986 volumes.

${ }^{2}$ International 1/4-inch rule.

${ }^{3} 1967$ sawtimber volume figues have been adjusted from those published after the 1967 survey to conform to 1986 data because of changes in Survey procedures and volume equations.
} 


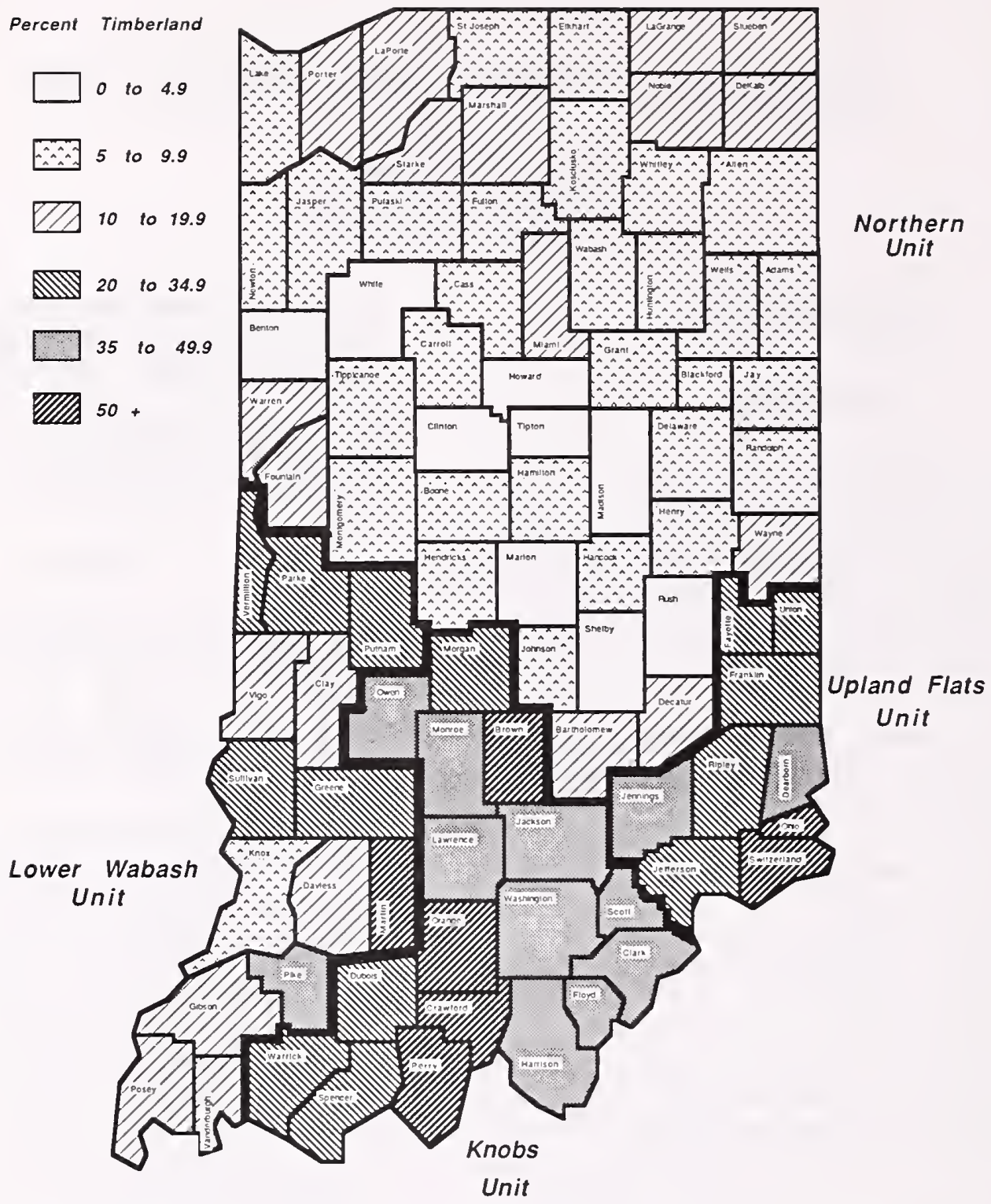

Figure 1.-Timberland as a percent of land area by county, Indiana, 1986.
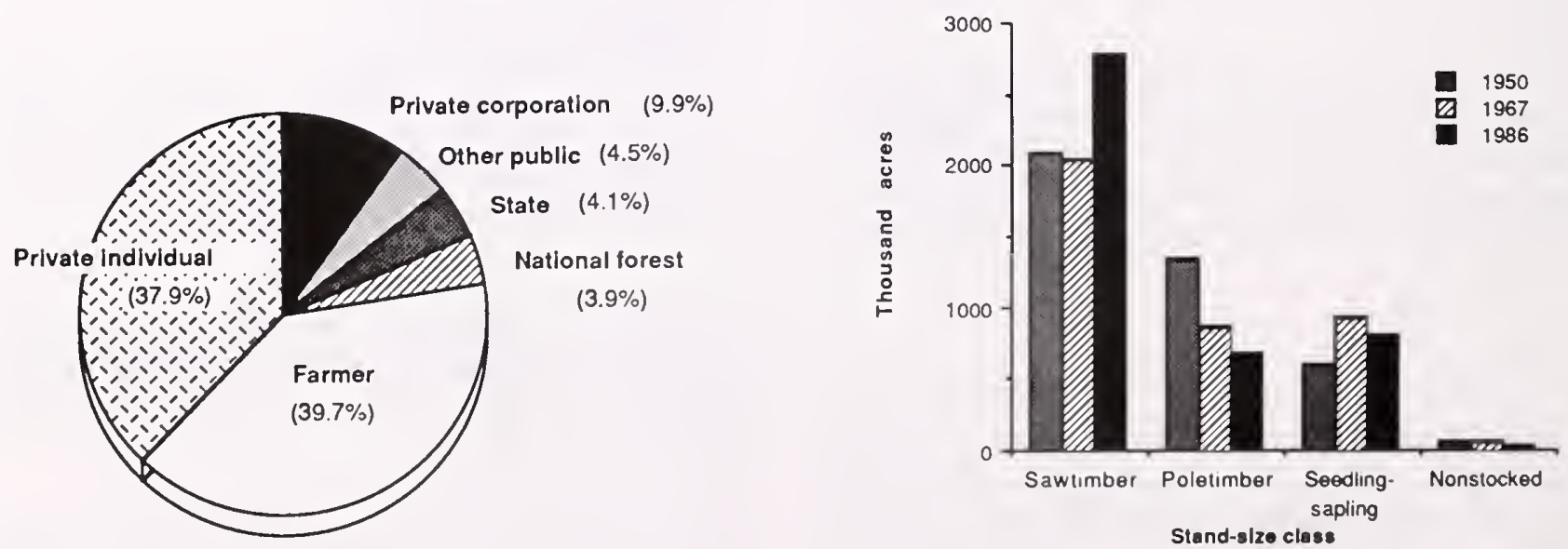

Figure 2.-Area of timberland by owner class, 1986.

Figure 3.-Area of timberland by stand-size class, 1950, 1967, and 1986. 


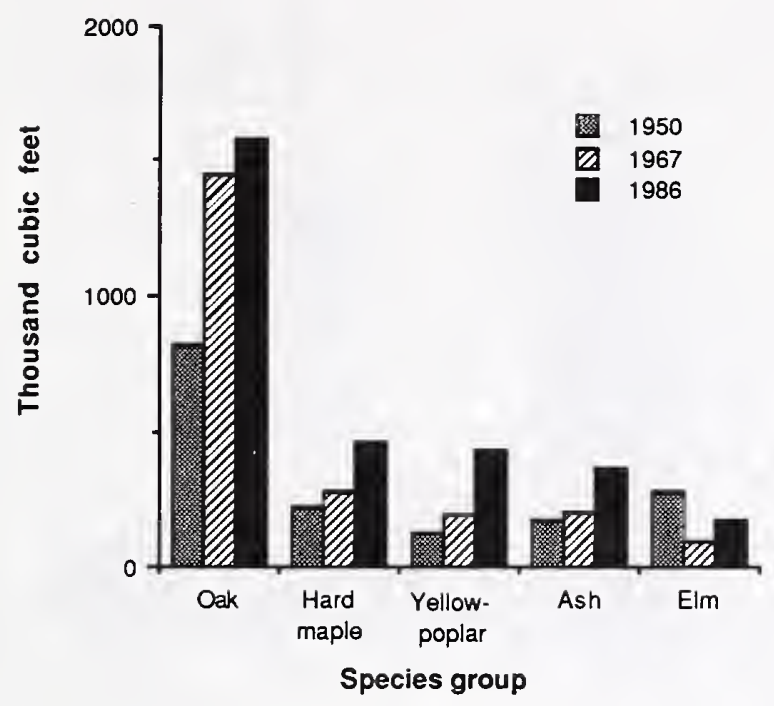

Figure 4.-Trend in growing-stock volume by selected species group.

- Growing-stock volume on timberland averaged 1,215 cubic feet per acre in 1986, compared to 938 cubic feet per acre in 1967, an increase of 30 percent.

- Sawtimber volume per acre increased 39 percent, from 3,212 board feet in 1967 to 4,475 board feet in 1986.

- The volume in live cull trees is 811 million cubic feet; salvable dead tree volume is 82 million cubic feet.

- Private parties (all non-public owners) own 85 percent ( 4.5 billion cubic feet) of the growing-stock volume.

- The oak-hickory forest type (which includes many non-oak species) contains 37 percent of the sawtimber volume ( 7.1 billion board feet).

\section{Stand Conditions}

- Net annual growth of growing-stock trees on timberland was 154 million cubic feet, 2.9 percent of inventory in 1985. In 1966, net annual growth of growing-stock trees was 104 million cubic feet, 2.8 percent of inventory.

- Net annual growth of growing-stock trees on timberland averaged 35.8 cubic feet per acre in 1985, up 35 percent from 1966 (26.6 cubic feet per acre) (fig. 5).

- Annual mortality of growing stock amounted to 37.5 million cubic feet, 0.7 percent of inventory in 1985.

- Net annual growth of sawtimber on timberland was 726 million board feet, 3.8 percent of inventory in 1985. In 1966, net annual growth of sawtimber was 270 million board feet, 2.2 percent of inventory.

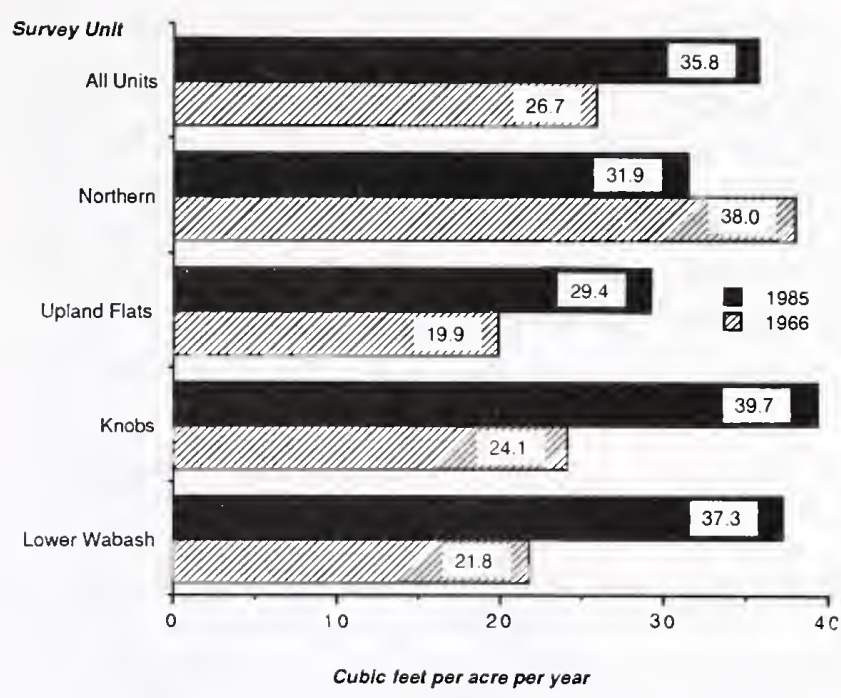

Figure 5.-Net growing-stock growth per acre, 1966 and 1985.

- Net annual growth of sawtimber trees on timberland averaged 169 board feet per acre in 1985 , nearly three times the rate in 1966 ( 69 board feet per acre). This dramatic increase in sawtimber growth was primarily due to ingrowth, the volume of trees that had not yet reached the merchantable diameter limit (9 inches for softwoods, 11 inches for hardwoods) in 1967 but had by 1986. This phenomenon is typical of a rapidly maturing second-growth forest.

- Annual mortality of sawtimber amounted to 101 million board feet, 0.5 percent of inventory in 1985 .

\section{Timber Use}

- Timber removals from growing stock totaled 93 million cubic feet in 1985 ( 1.8 percent of inventory and 65 percent of growth).

- Sixty percent of the growing-stock removals were for saw logs (fig. 6).

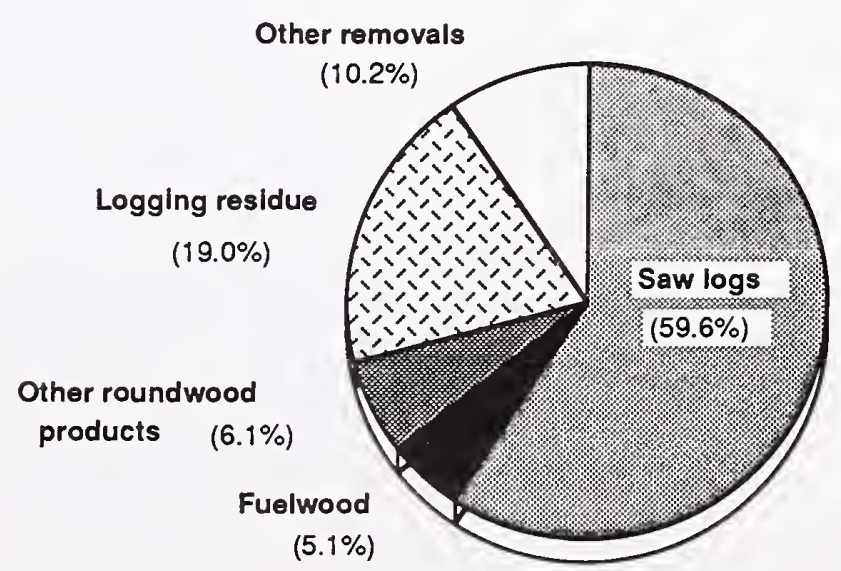

Figure 6.-Timber removals from growing stock, 1985. 
- Sawtimber removals totaled 462 million board feet in 1985 (2.4 percent of inventory and 63 percent of growth).

- Oak and hickory growing-stock removals (49 million cubic feet) slightly exceed growth (48 million cubic feet); but removals in all other species (44 million cubic feet) were less than half of growth (94 million cubic feet) (fig. 7).

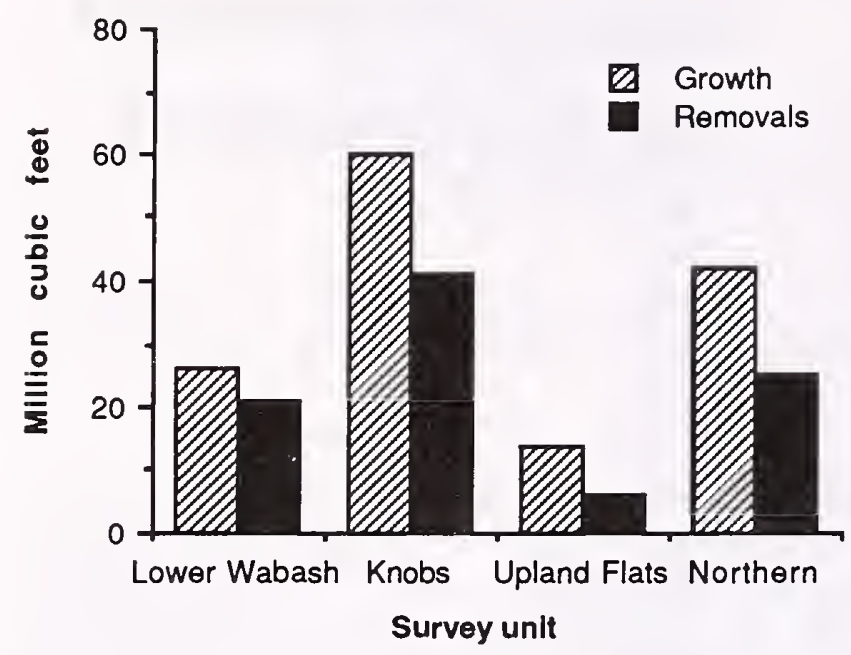

Figure 7.-Growth and removals on timberland, 1985.

- The oaks accounted for 46 percent (212 million board feet) of the sawtimber removals in 1985 (3.1 percent of oak inventory and 105 percent of oak growth).

- Fuelwood production topped 693 thousand cords in 1984. However, only 10 percent came from growing stock; the rest came from rough, rotten, and dead trees ( 22 percent), plant residues ( 27 percent), and other sources (41 percent), primarily tops and limbs of harvested trees and trees from nonforest land (fig. 8).

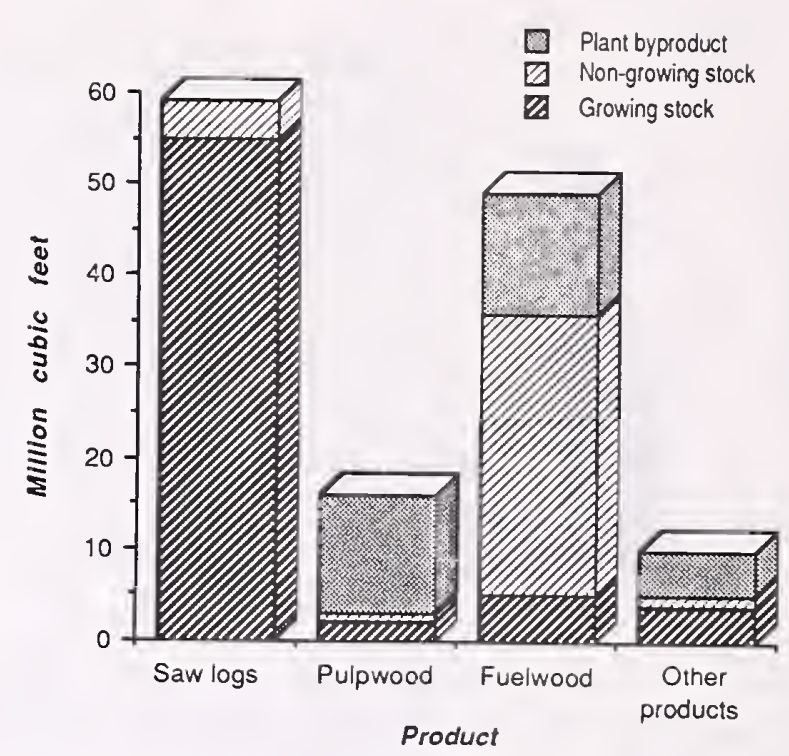

Figure 8.-Timber products output by source of material, 1984.

\section{Biomass}

- Live tree biomass (trees greater than 1 inch in d.b.h.) totaled 328 million green tons (an average of 76.3 green tons per acre) in 1986 .

- Seventy-three percent (241 million green tons) of all live tree biomass was in growing-stock trees 5 inches d.b.h. and larger (fig. 9).

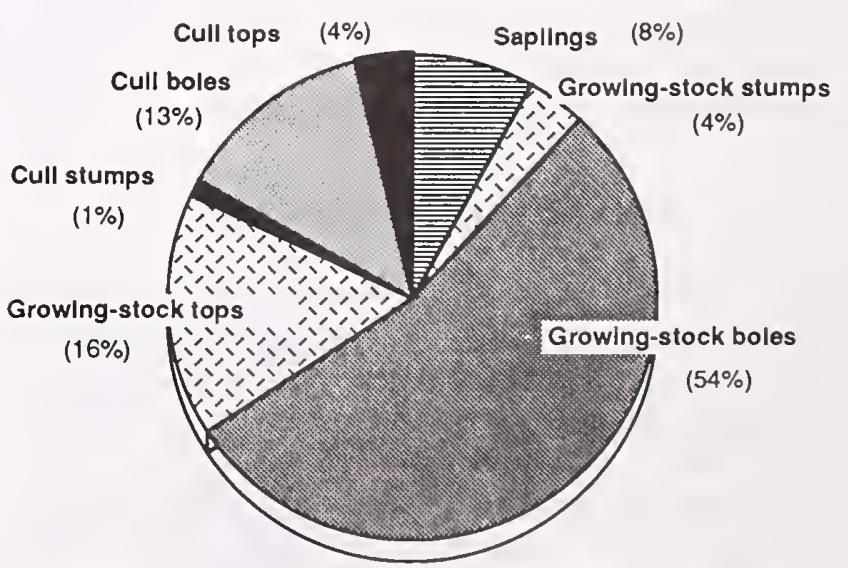

Figure 9.-Components of timberland biomass. 


\section{APPENDIX}

\section{ACCURACY OF SURVEY}

Forest Inventory and Analysis information is based on a sampling procedure designed to provide reliable statistics at the State and Survey Unit levels. Consequently, the reported figures are estimates only. A measure of reliability of these figures is given by sampling errors. These sampling errors mean that the chances are two out of three that if a 100-percent inventory had been taken, using the same methods, the results would have been within the limits indicated.

For example, the estimated growing-stock volume in the State in 1986, 5,217.9 million cubic feet, has a sampling error of +1.57 percent $(+81.9$ million cubic feet). The growing-stock volume from a 100-percent inventory would be expected to fall between 5,136.0 and 5,299.8 million cubic feet $(5,217.9$ +81.9 ), there being a one in three chance that this is not the case.

The following tabulation shows the sampling errors for the 1986 Indiana Forest Inventory:

$\begin{array}{lcc}\begin{array}{l}\text { Item } \\ \text { Growing stock }\end{array} & \begin{array}{c}\text { State totals } \\ \text { (Million cubic feet) }\end{array} & \begin{array}{c}\text { Sampling error } \\ \text { (Percent) }\end{array} \\ \quad \text { Volume (1986) } & 5,217.9 & 1.57 \\ \text { Growth (1985) } & 142.1 & 3.42 \\ \quad \begin{array}{c}\text { Average annual } \\ \text { removals (1966-1985) }\end{array} & 72.6 & 5.40 \\ \begin{array}{c}\text { Sawtimber } \\ \quad \text { Volume (1986) }\end{array} & \begin{array}{c}\text { (Million board feet) } \\ \text { Growth (1985) }\end{array} & 1.824 .2 \\ \quad \begin{array}{c}\text { Average annual } \\ \text { removals (1966-1985) }\end{array} & 729.1 & 5.47 \\ \text { Timberland } & 314.0 & \\ \quad \text { Area (1986) } & \text { (Thousand acres) } & 5.68 \\ \quad 4,295.8 & 1.00\end{array}$

As survey data are broken down into sections smaller than Survey Unit totals, the sampling error increases. For example, the sampling error for timberland area in a particular county is higher than that for total timberland area in the Unit. Table 89 shows the sampling errors for Unit and county totals. To use this table for data smaller than county totals use the following formula to compute error estimates:

$$
\mathrm{E}=\frac{(\mathrm{SE}) \sqrt{(\text { Unit total area or volume }}}{\sqrt{\text { (Volume or area smaller than Unit total) }}}
$$

where: $\mathrm{E}=$ sampling error in percent

$$
\mathrm{SE}=\text { Unit total error from table } 90 \text { for area }
$$
or volume.

For example, to compute the error on the area of oak hickory type in Jackson County, proceed as follows:

Area of oak-hickory type in Jackson County from table $12=50,300$ acres

Area of all timberland in the Knobs Unit from table $12=1,741,100$ acres

Unit total error for area in the Knobs Unit from table $90=0.83$ percent

Using the formula above:

$$
\begin{aligned}
\text { Error } & =\frac{(0.83) \sqrt{1,741,100}}{\sqrt{50,300}} \\
& =4.88 \text { percent }
\end{aligned}
$$

\section{SURVEY PROCEDURES}

Two-phase sampling using both new and remeasurement ground plots was used in this inventory.

The major steps in the Indiana Forest Inventory were as follows:

\section{Aerial photography (Phase 1)}

Aerial photographs of the entire area were obtained from the Hoosier National Forest and USDA Agricultural Stabilization and Conservation Service. Nine-inch square, black and white Panchromatic prints on a scale of 1:40,000 were used throughout the entire State. Approximate date of photography for each county is shown in table 1. For those counties with photos taken prior to 1980, National High Altitude Photography (NHAP) photos were used to verify change information. The NHAP photos were taken between 1981 and 1983 .

The locations of the plots used in the 1967 inventory were transferred to these new photographs. Photographs were assembled into township mosaics, and a systematic grid of 121 one-acre points (each point representing approximately 190.4 acres) was overlaid on each township mosaic. Each of these points (both the new systematic grid points and the 
Table 1..-Oates of aerial photography by county and Forest 5urvey Unit, Indiana

\begin{tabular}{|c|c|c|c|}
\hline Unit and County & Oa te & Unit and County & Date \\
\hline \multicolumn{2}{|l|}{ Lower Wabash Unit } & Northern Unit & \\
\hline Clay & $10-80$ & Adams & $4-83$ \\
\hline Oaviess & $9-74$ & Allen & $5-81$ \\
\hline Gibson & $10-74$ & Bartholomew & $11-80$ \\
\hline Greene & $10-80$ & Benton & $6-83$ \\
\hline knox & $4-78$ & Blackford & $9-80$ \\
\hline Martin & 9.74 & Boone & $5-72$ \\
\hline Parke & $6-71$ & Carroll & $11-81$ \\
\hline Pike & $9-74$ & Cass & $4-82$ \\
\hline Posey & $6-80$ & $\mathrm{Cl}$ inton & $5-72$ \\
\hline Putnam & $4-78$ & Decatur & $10-81$ \\
\hline Sullivan & $9-74$ & Dekalo & $6-72$ \\
\hline Vanderburgh & $6-80$ & Delaware & $6-80$ \\
\hline Vermillion & $9-72$ & El khart & $5-81$ \\
\hline \multirow[t]{5}{*}{ Vigo } & $9-74$ & Founta in & $6-83$ \\
\hline & & Fulton & $10-80$ \\
\hline & & Grant & $6-80$ \\
\hline & & Hamil ton & $5-82$ \\
\hline & & Hancock & $5-80$ \\
\hline Knobs Unit & & Hendricks & $4-78$ \\
\hline 8rown & $10-80$ & Henry & $6-80$ \\
\hline Clark & $10-81$ & Howard & $5-84$ \\
\hline Crawford & $11-79$ & Huntington & $4-82$ \\
\hline Oubois & $11-79$ & Jasper & $4-84$ \\
\hline Floyd & $6-80$ & Jay & $9-80$ \\
\hline Harrison & $6=80$ & Johns on & $9-72$ \\
\hline Jackson & $10-80$ & Kosciusko & $7-73$ \\
\hline Lawrence & $9-79$ & LaGrange & $10-74$ \\
\hline Monroe & $10-80$ & Lake & $9-73$ \\
\hline Morgan & $10-80$ & Laporte & $11-81$ \\
\hline Orange & $9-79$ & Madison & $6-80$ \\
\hline Owen & $10-80$ & Marion & $8-71$ \\
\hline Perry & $11-79$ & Marshall & $7-73$ \\
\hline $5 \cot t$ & $10-80$ & Miami & $10-80$ \\
\hline Spencer & $9-74$ & Montgomery & $7-71$ \\
\hline Warrick & $6-80$ & Newton & $6-82$ \\
\hline \multirow[t]{5}{*}{ Washington } & $10-80$ & Noble & 7.73 \\
\hline & & Porter & 6.82 \\
\hline & & Pulaski & $5-81$ \\
\hline & & Randol ph & 9.80 \\
\hline & & Rush & $10-81$ \\
\hline Upland Flats Unit & & 5t. Joseph & $10-80$ \\
\hline Oearborn & $7-80$ & Selby & $6-80$ \\
\hline Fayet te & $10-81$ & 5tarke & $5-81$ \\
\hline Frankl in & $10-71$ & 5tueben & $7-73$ \\
\hline Jefferson & $10-80$ & Tippecanoe & $6-71$ \\
\hline Jennings & $10-81$ & Tipton & $6-72$ \\
\hline Ohio & $7-80$ & Wabash & $6-72$ \\
\hline Ripley & $10-81$ & Warren & $6-83$ \\
\hline 5witzerland & $7-80$ & Wayne & $10-80$ \\
\hline \multirow[t]{3}{*}{ Union } & $10-81$ & Wells & $6-72$ \\
\hline & & White & $4-82$ \\
\hline & & Whitley & $6-72$ \\
\hline
\end{tabular}

old sample plots) was examined by aerial photogrammetrists and classified stereoscopically based on land use. If trees were present, forest type and stand sizedensity class were recorded. Then all the old sample locations and a sample of the new points were sent to the field for the field crew to verify the photo classification and to take further measurements. A total of 126,629 points (120,949 new and 5,680 old) was examined stereoscopically (table 2 ).

\begin{tabular}{|c|c|c|c|c|c|}
\hline \multirow[b]{2}{*}{ Photo land class } & \multirow[b]{2}{*}{$\begin{array}{l}\text { Ali } \\
\text { Units }\end{array}$} & \multicolumn{4}{|c|}{ Forest Survey Unit } \\
\hline & & $\begin{array}{r}\text { Lower } \\
\text { wabash }\end{array}$ & Knobs & $\begin{array}{r}\text { Upland } \\
\text { Fiats } \\
\end{array}$ & Northern \\
\hline $\begin{array}{l}\text { Timberland } \\
\text { Reserved timberland } \\
\text { Questionable } \\
\text { Nonforest with trees } \\
\text { Nonforest without trees } \\
\text { Water }\end{array}$ & $\begin{array}{r}24,462 \\
830 \\
645 \\
3,842 \\
95,572 \\
1,278 \\
\end{array}$ & $\begin{array}{r}4,794 \\
61 \\
90 \\
871 \\
13.931 \\
285 \\
\end{array}$ & $\begin{array}{r}9,665 \\
223 \\
307 \\
639 \\
11.834 \\
265 \\
\end{array}$ & $\begin{array}{r}2.840 \\
176 \\
73 \\
454 \\
5.423 \\
77 \\
\end{array}$ & $\begin{array}{r}7.163 \\
370 \\
175 \\
1.878 \\
64.384 \\
651 \\
\end{array}$ \\
\hline All classes & 126.629 & 20.032 & 22,933 & 9,043 & 74,621 \\
\hline
\end{tabular}

\section{Plot measurements (Phase 2)}

Each plot location was visited on the ground by a Forest Service field crew. They classified the plot based on its current land use and recorded various other descriptive information. Table 3 summarizes the results of this step of the inventory.
Table 3...-Number of ground plots by ground land use class. and forest Survey Unit, Indiana, 1986

\begin{tabular}{|c|c|c|c|}
\hline $\begin{array}{l}\text { Survey Unit and } \\
\text { ground land use class }\end{array}$ & $\begin{array}{l}\text { Old plots } \\
\text { remeasured }\end{array}$ & $\begin{array}{l}\text { New plots } \\
\text { established }\end{array}$ & $\begin{array}{l}\text { Total yround } \\
\text { plots taken }\end{array}$ \\
\hline \multicolumn{4}{|l|}{ All Units } \\
\hline Timberland & 1,059 & 939 & 1,998 \\
\hline Reserved timberland & & 115 & 142 \\
\hline Nonforest with trees & 230 & 228 & 458 \\
\hline Nonforest without trees & 4,308 & 4,416 & 8.724 \\
\hline Water & 61 & 57 & 118 \\
\hline Total & 5.685 & 5,755 & 11,440 \\
\hline \multicolumn{4}{|l|}{ Lower Wabash Unit } \\
\hline Timberl and & 205 & 208 & 413 \\
\hline Reserved timberland & 6 & 12 & 18 \\
\hline Nonforest with trees & 55 & 43 & 98 \\
\hline Nonforest without trees & 600 & 648 & 1,248 \\
\hline water & 14 & 12 & 26 \\
\hline Total & 880 & 923 & 1,803 \\
\hline \multicolumn{4}{|l|}{ Knobs Unit } \\
\hline Timberland & 460 & 409 & 869 \\
\hline Reserved timberland & 12 & 45 & 57 \\
\hline Nonforest with trees & 65 & 62 & 127 \\
\hline Nonforest without trees & 503 & 525 & 1.028 \\
\hline water & 16 & 16 & 32 \\
\hline Total & 1,056 & 1.057 & 2.113 \\
\hline \multicolumn{4}{|l|}{ Upland Flats Unit } \\
\hline Timberland & 127 & 121 & 248 \\
\hline Reserved timberland & 0 & & 37 \\
\hline Nonforest with trees & 27 & 36 & 63 \\
\hline Nonforest without trees & 219 & 226 & 445 \\
\hline water & 7 & 2 & 9 \\
\hline Total & 380 & 422 & 802 \\
\hline \multicolumn{4}{|l|}{ Northern Unit } \\
\hline Timberland & 267 & 201 & 468 \\
\hline Reserved timberland & 9 & 21 & 30 \\
\hline Nonforest with trees & 83 & 87 & 170 \\
\hline Nonforest without trees & 2,986 & 3,017 & $6 ; 003$ \\
\hline water & 24 & 27 & 51 \\
\hline Total & 3,369 & 3,353 & 6.722 \\
\hline
\end{tabular}

On those plots classified as timberland, wooded pasture, or windbreak (at least 120 feet wide), a ground plot was established or remeasured. The ground plot consisted of a 10-point cluster covering approximately 1 acre. At each point, trees 5.0 inches or more in d.b.h. were sampled on a 37.5 Basal Area Factor (BAF) variable-radius plot, and trees less than 5.0 inches d.b.h. were sampled on a $1 / 300$-acre fixedradius plot.

\section{Area estimates}

Area estimates outside the Hoosier National Forest were made using two-phase estimation methods.

In this type of estimation, a preliminary estimate of area by land use is made from the aerial photographs (phase 1) and corrected by the plot measurements (phase 2). A complete description of this estimation method is presented by Loetsch and Haller, 1964. Estimates of area for a particular county are based on the aerial photo points taken in that county, corrected using all the ground plots in the Survey Unit, regardless of the county in which they were taken. This was done because there were not enough ground plots in any one county to accurately correct the aerial photo interpretation in that county. Unit-wide correction rates should be accurate at

${ }^{4}$ Loetsch, F; Haller, K. E. 1964. Forest inventory. Volume 1: Statistics of forest inventory and information from aerial photographs. BLV Verlagsgesellschaft Munch Basle Vienna. 436 p. 
the county level because we have made every effort to ensure that the plot interpretation is consistent throughout each Survey Unit.

Area estimates within the Hoosier National Forest were obtained from compartment examination records maintained by the Forest Timber Management Staff. This is an intensive area inventory system in which, over a period of years, each stand in the Hoosier is mapped on aerial photographs and then classified by ground visits.

\section{Volume estimates}

Estimates of volume per acre are made from the trees measured on the 10-point plots. Estimates of volume per acre were multiplied by the area estimates to obtain estimates of total volume. Net cubic foot volumes are based on equations developed by Smith and Weist $1982^{5}$ for use in Indiana. Board foot International 1/4-inch, board foot Doyle (table 4 ), and cubic foot volume in saw $\log$ (table 5) estimates are developed based on factors derived from full tree

\begin{tabular}{|c|c|c|c|c|}
\hline \multirow{2}{*}{$\begin{array}{l}0.8 . H . \\
\text { (inches) }\end{array}$} & \multicolumn{2}{|c|}{ International $1 / 4$-inch rule } & \multicolumn{2}{|c|}{ Doyle rule } \\
\hline & Softwoods & Mardwoods & Softwoods & Hardwoods \\
\hline $9.0-10.9$ & 5.1587 & 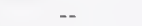 & 1.8300 & -- \\
\hline $11.0-12.9$ & 5.4688 & 4.9076 & 2.6526 & 2.1085 \\
\hline $13.0-14.9$ & 5.6519 & 5.1315 & 3.4080 & 2.6800 \\
\hline $15.0-16.9$ & 5.7234 & 5.2444 & 3.9749 & 3.1258 \\
\hline $17.0-18.9$ & 5.8094 & 5.2718 & 4.5314 & 3.5071 \\
\hline $19.0-20.9$ & 5.6641 & 5.1770 & 4.7697 & 3.7644 \\
\hline $21.0-22.9$ & 5.6942 & 5.1096 & 4.9565 & 4.0580 \\
\hline $23.0-24.9$ & 5.3753 & 4.8778 & 5.1949 & 4.2229 \\
\hline $25.0-26.9$ & 5.3753 & 4.8778 & 5.9153 & 4.3566 \\
\hline $27.0-28.9$ & 5.3753 & 4.8778 & 5.3148 & 4.4843 \\
\hline $29.0+$ & 5.3753 & 4.8778 & 5.8066 & 4.9115 \\
\hline
\end{tabular}

Table 5.--Factors to determine saw log volume as a proportion of growing-stock bole volume by diameter class and softwoods and hardwoods

\begin{tabular}{rcc}
\hline $\begin{array}{l}\text { D.B.H. } \\
\text { (inches) }\end{array}$ & Softwoods & Hardwoods \\
\hline $9.0-10.9$ & 0.8835 & -- \\
$11.0-12.9$ & .9533 & 0.7495 \\
$13.0-14.9$ & .9530 & .7905 \\
$15.0-16.9$ & .9488 & .8006 \\
$17.0-18.9$ & .9424 & .8048 \\
$19.0-20.9$ & .9016 & .7809 \\
$21.0-22.9$ & .8842 & .7691 \\
$23.0-24.9$ & .7918 & .7361 \\
$25.0-26.9$ & .7918 & .7361 \\
$27.0-28.9$ & .7918 & .7361 \\
$29.0+$ & .7918 & .7361 \\
\hline
\end{tabular}

${ }^{5}$ Smith, W. Brad; Weist, Carol A. 1982. A net volume equation for Indiana. Resour. Bull. NC-63. St. Paul, MN: U.S. Department of Agriculture, Forest Service, North Central Forest Experiment Station. 7 p. measurements taken throughout the Central States (Illinois, Indiana, Iowa, and Missouri) and an equation developed by Wiant and Castenaeda $1977^{6}$. Biomass estimates are based on equations developed by Smith 1985 ?

\section{Growth and mortality estimates}

On remeasurement plots, estimates of growth and mortality per acre come from the remeasured diameters of trees and from observation of trees that died between inventories. Growth is reported for 1985 , the last year before the inventory, and is based on an assumption of constant basal area growth over the remeasurement period. Mortality is reported for 1985 also, and is based on an assumption of constant volume mortality over the remeasurement period.

On new plots, where trees were not remeasured, estimates of growth and mortality were obtained by using the Central States Stand and Tree Evaluation and Modeling System (STEMS) ${ }^{8}, 9$ to project the growth and mortality of trees for 1 year. The STEMS growth model was adjusted to meet local conditions, using the data from the remeasured plots and a method developed by Smith $1983^{10}$. As with volume, total growth and mortality estimates were obtained by multiplying the per acre estimates by area estimates.

${ }^{6}$ Wiant, Harry V., Jr.; Castenaeda, Froylan. 1977. Mesavage and Girard's volume tables formulated. BLM4. Denver, CO: U.S. Department of the Interior, Bureau of Land Management, Denver Service Center: 1-4.

${ }^{7}$ Smith, W. Brad. 1985. Factors and equations to estimate forest biomass in the North Central Region. Res. Pap NC-268. St. Paul, MN: U.S. Department of Agriculture, Forest Service, North Central Forest Experiment Station. 6 p.

${ }^{8}$ Miner, Cynthia L; Walters, Nancy R. 1984. STEMS: a nontechnical description for foresters. Res. Pap NC-252. St. Paul, MN: U.S. Department of Agriculture, Forest Service, North Central Forest Experiment Station. 12 p.

${ }^{9}$ Shifley, S. F. 1987. A generalized system of models forecasting central states tree growth. Res. Pap NC-279. St. Paul, MN: U.S. Department of Agriculture, Forest Service, North Central Forest Experiment Station. 10 p.

${ }^{10}$ Smith, W. Brad. 1983. Adjusting the STEMS regional growth models to improve local predictions. Res. Note NC-297. St. Paul, MN: U.S. Department of Agriculture, Forest Service, North Central Forest Experiment Station. $5 \mathrm{p}$. 
6. Average annual removals estimates

Average annual growing-stock and sawtimber removals (1966 to 1985) were estimated only from the remeasurement plots. These estimates are obtained from trees measured in the last survey and cut or otherwise removed from the timberland base. New plots were not used to estimate removals. Because the remeasurement plots made up only half of the total ground plots, average annual removals estimates have greater sampling errors than volume and growth estimates.

7. Timber removals, utilization, and timber product output estimates

Statistics on timber product output during 1984 came from canvassing (with a formal questionnaire) all the known primary wood-using mills that consume Indiana logs and bolts. Indiana Department of Natural Resources (IDNR) foresters personally canvassed all the known Indiana primary mills (except one pulpmill). IDNR utilization and marketing specialists provided estimates based on prior knowledge and contacts for a few mills that did not furnish complete data.

The North Central Forest Experiment Station mailed a formal questionnaire to the only Indiana pulpmill and all known out-of-State mills using Indiana roundwood. Follow-up on nonrespondents was by mail and telephone.

A sample of Illinois households and fuelwood producers provided estimates of fuelwood and post production in Illinois during 1983. Fuelwood and fencepost output in Indiana for 1984 was estimated by extrapolating the study results in Illinois to Indiana.

Wood utilization factors for converting timber products output to removals from growing stock were obtained during a 1984-1985 utilization study in Illinois, a 1971-1972 utilization study in Missouri, a 1966 utilization study in Indiana, and a 1964-1965 utilization study in Michigan.

Because this was a 100-percent sample of all primary wood-using mills, there is no sampling error reported for 1985 removals and timber products. This is not to say that the estimates reported here are totally accurate. Sampling error is a measure of precision, not accuracy, and tells in what range we would expect to obtain an estimate were we to repeat the procedure on a new sample. Because we sampled all mills, we would expect the same results if we repeated the procedure.

\section{COMPARING INDIANA'S THIRD INVENTORY WITH THE SECOND INVENTORY}

Data from new forest inventories are often compared with data from earlier inventories to determine trends in forest resources. However, changes in procedures and definitions between surveys often make it necessary to adjust earlier survey data so that they are comparable to data from the new survey. A consistency check was made for the Indiana inventory to ensure that the changes observed between inventories reflect actual changes in the resource and not changes in definitions or procedures.

\section{Identifying and Correcting Procedural Changes}

Between the 1967 and 1986 inventories of Indiana, some procedural changes were made in the method of deriving annual growth and mortality estimates and determining forest type. Also, different volume equations and forest types were used for the two inventories.

New volume equations were developed for Indiana, and these equations were used to compute the 1986 volumes and also to recompute the 1967 volume. The recomputed 1967 growing-stock volume averaged 4.3 percent greater than that reported in the 1967 report. Volumes for 1967 shown in this report are the recomputed volumes based on new volume equations and observations from the 1967 inventory.

Mortality figures published in the 1967 inventory report were based on field estimates from nonremeasurement plots. Information gathered on remeasurement plots during the current inventory was used to adjust the 1967 mortality figures. This adjustment, together with the new volume equations, also changed the estimate of net growth for the 1967 inventory.

In the 1967 report, fewer forest types were identified than in this report; however, all the types used in this report are subsets of forest types used in the old report. Areas and volumes in the oak-hickory type in 1967 will be in either the oak-hickory, chestnutscarlet oak, or sassafras-persimmon type in 1986. Areas and volumes in the oak-gum-cypress type in 1967 will be in either the oak-gum or lowland oak type in 1986. Similarly, the 1967 elm-ash-cottonwood type is made up of the elm-ash-soft maple and cottonwood types in 1986; and the 1967 maple-beech- 
birch type is made up of the maple-beech and cherryash-yellow-poplar types in 1986 . We could not classify the 1967 area by the 1985 types, so all comparisons must be made by combining 1986 data to reflect the 1967 types.

\section{Checking for Consistency}

A test was made to ensure that it was possible to move from the adjusted 1967 resource statistics to the 1986 values by means of a computer program for updating and projecting timber volume, growth, and removals. Using the adjusted 1967 volume, growth rates, and removals rates for the period between the two surveys, the program projected the inventory from 1967 to 1986 . The program outputs volume, net growth, and removals of growing stock for every year in the period. Thus, inconsistencies in volume, growth, and removals were identified and resolved.

This program estimates how volume, growth, and removals could have logically changed over the inventory period to be consistent with the estimates of past and current volume, growth, and removals and average annual removals presented in this report. The growing-stock growth used for this program was increased by 6.1 million cubic feet per year in both 1967 and 1986 to account for nontimberland that converted to timberland. Between 1967 and 1986, we found that about 900,000 acres of nontimberland converted to timberland. The current growing-stock volume on this land is 514 million cubic feet. Removals did not require adjustment because they already include "other" removals (see Definition of Terms in Appendix), which includes the volume of timber on land that converted from timberland to nontimberland. Between 1967 and 1986, we found that about 500,000 acres of timberland converted to nontimberland. The growing-stock volume on this land was 400 million cubic feet in 1966.

\section{LOG GRADE}

In Indiana the butt log of every sawtimber sample tree was graded for quality. Additionally, all logs in a smaller sample of trees throughout the State were graded. The volume yield by log grade for each tree in the latter sample was used to distribute the volume of trees in the former sample into log-grade classes by species group. The resulting volumes by log-grade classes were expanded to provide an estimate for the entire State.

Logs were graded on the basis of external characteristics as indicators of quality. Hardwood species were graded according to "A guide to hardwood log grading" $1973^{11}$. The best 12 -foot section of the lowest 16 -foot hardwood log, or the best 12 -foot upper section if the butt log did not meet minimum log-grade standards, was graded as follows:

\footnotetext{
${ }^{11}$ Rast, Everette D.; Sonderman, David L.; Gammon, Glenn L. 1973. A guide to hardwood log grading Gen. Tech. Rep NE-1. Upper Darby, PA: U.S. Department of Agriculture, Forest Service, Northeastern Forest Experiment Station. $31 \mathrm{p}$.
} 


\section{Forest Service standard grades for hardwood factory saw logs}

\begin{tabular}{|c|c|c|c|c|c|c|c|c|c|}
\hline \multirow{2}{*}{\multicolumn{2}{|c|}{ Grading factors }} & \multicolumn{8}{|c|}{ Specifications } \\
\hline & & \multicolumn{3}{|c|}{ Log grade 1} & \multicolumn{4}{|c|}{ Log grade 2} & \multirow{2}{*}{\begin{tabular}{|c|} 
Log grade 3 \\
$\begin{array}{c}\text { Butts and } \\
\text { uppers }\end{array}$
\end{tabular}} \\
\hline \multicolumn{2}{|l|}{ Position in tree } & $\begin{array}{c}\text { Butts } \\
\text { only }\end{array}$ & \multicolumn{2}{|c|}{$\begin{array}{c}\text { Butts and } \\
\text { uppers }\end{array}$} & & \multicolumn{3}{|c|}{ Butts and uppers } & \\
\hline \multicolumn{2}{|c|}{ Scaling diameter, inches } & $113-15$ & $16-19$ & $20+$ & $211+$ & & $12+$ & & $8+$ \\
\hline \multicolumn{2}{|c|}{ Length without trim, feet } & & $10+$ & & $10+$ & $8-9$ & $10-11$ & $12+$ & $8+$ \\
\hline \multirow{3}{*}{$\begin{array}{l}\text { Required } \\
\text { clear cuttings }{ }^{3} \\
\text { of each of three } \\
\text { best faces }\end{array}$} & Min. length, feet & 7 & 5 & 3 & 3 & 3 & 3 & 3 & 2 \\
\hline & Max. number & 2 & 2 & 2 & 2 & 2 & 2 & 3 & No Limit \\
\hline & $\begin{array}{l}\text { Min. proportion } \\
\text { of log length } \\
\text { required in } \\
\text { clear cutting }\end{array}$ & $5 / 6$ & $5 / 6$ & $5 / 6$ & $2 / 3$ & $3 / 4$ & $2 / 3$ & $2 / 3$ & $1 / 2$ \\
\hline \multirow{2}{*}{$\begin{array}{l}\text { Maximum } \\
\text { sweep and crook } \\
\text { allowance }\end{array}$} & $\begin{array}{l}\text { For logs with less } \\
\text { than one-fourth } \\
\text { of end in sound } \\
\text { defects }\end{array}$ & \multicolumn{3}{|c|}{15 percent } & & \multicolumn{3}{|c|}{30 percent } & 50 percent \\
\hline & $\begin{array}{l}\text { For logs with more } \\
\text { than one-fourth } \\
\text { of end in sound } \\
\text { defects }\end{array}$ & \multicolumn{4}{|c|}{10 percent } & \multicolumn{3}{|c|}{20 percent } & 35 percent \\
\hline
\end{tabular}

Maximum scaling deduction

40 percent $^{5}$

50 percent $^{6}$

50 percent

'Ash and basswood butts can be 12 inches if they otherwise meet requirements for small \#1's.

'Ten-inch logs of all species can be \#2's if they otherwise meet requirements for small \#1's.

${ }^{3} \mathrm{~A}$ clear cutting is a portion of a face, extending the width of the face, that is free of defects.

${ }^{4} \mathrm{~A}$ face is one-fourth of the surface of the $\log$ as divided lengthwise.

${ }^{5}$ Otherwise \#1 logs with 41-60 percent deductions can be \#2.

${ }^{6} 0$ therwise \#2 logs with 51-60 percent deductions can be \#3 


\section{Forest Service standard specifications for hardwood construction logs (tie and timber logs) ${ }^{1}$}

\begin{tabular}{ll}
\hline Position in tree & Butt and upper \\
\hline Min. diameter, small end & 8 inches + \\
\hline Min. length, without trim & 8 feet \\
\hline Clear cuttings & No requirements. \\
\hline Sweep allowance, absolute & $\begin{array}{l}\text { One-fourth of the diameter at the small end for each } 8 \text { feet of } \\
\text { length. }\end{array}$
\end{tabular}

\section{Single knots}

Sound surface defects
Whorled knots

Any number, if no one knot has an average diameter above the callus in excess of one-third of the log diameter at point of occurrence.

Any number if sum of knot diameters above the callus does not exceed one-third of the log diameter at point of occurrence.

Holes

Any number provided none has a diameter over one-third of the log diameter at point of occurrence, and none extends more than 3 inches into included timber. ${ }^{2}$

Same requirements as for sound defects if they extend into included timber. ${ }^{2}$ No limit if they do not.

No requirements.

None allowed; log must be sound internally, but will admit one shake not to exceed one-fourth the scaling diameter and will admit a longitudinal split not extending more than 5 inches into the contained timber.

${ }^{1}$ These specifications are minimum for the class. If, from a group of logs, factory logs are selected first, thus leaving only nonfactory logs from which to select construction logs, then the quality range of the construction logs so selected is limited, and the class may be considered a grade. If selection for construction logs is given first priority, it may be necessary to subdivide the class into grades.

2Included timber is always square, and dimension is judged from small end.

Softwood species were graded according to the following specifications on the following page. 


\section{Log Grades for Eastern White Pine}

\begin{tabular}{|c|c|c|c|c|c|c|}
\hline $\begin{array}{l}\text { Log } \\
\text { grade }\end{array}$ & $\begin{array}{c}\text { Minimu } \\
\text { Diameter }\end{array}$ & $\begin{array}{l}\text { m size } \\
\text { Length' }\end{array}$ & $\begin{array}{l}\text { Sweep } \\
\text { or crook } \\
\text { allowance }\end{array}$ & $\begin{array}{l}\text { Total cull } \\
\text { allowance } \\
\text { including } \\
\text { sweep }\end{array}$ & $\begin{array}{l}\text { Maximum } \\
\text { weevil } \\
\text { injury }\end{array}$ & Allowable knot size (inches) ${ }^{2}$ on three best faces or minimum clearness on four faces \\
\hline \multirow{3}{*}{1} & (Inches) & (Feet) & \multicolumn{2}{|c|}{ (Percent) } & (Number) & (Inches) \\
\hline & $12 \& 13$ & $8-16$ & 20 & 50 & 0 & Four faces clear full length \\
\hline & $14+$ & $10-16$ & 20 & 50 & 0 & $\begin{array}{l}\text { Two faces clear full length, or four faces clear } 50 \text { percent length ( } 6 \text { feet min. } \\
\text { length) }\end{array}$ \\
\hline 2 & $6+$ & $8-16$ & 30 & 50 & 0 & $\begin{array}{l}\text { Sound knots } 1 . e^{4} \\
D / 6 \text { and less than } 3 \text { inches. } \\
\text { Unsound knots: } 1 . \text { e. } 1 \frac{1}{2} \text { inches and for: butt, logs } 1 . e . D / 12 \text {, upper logs } \\
\text { 1.e.D/10, or four faces clear } 50 \text { percent of length }\end{array}$ \\
\hline 3 & $6+$ & $8-16$ & 40 & 50 & $\begin{array}{l}\text { 8-foot logs: } \\
1 \text { weevil } \\
\text { 10-foot }+ \\
\text { logs: } \\
2 \text { weevils }\end{array}$ & $\begin{array}{l}\text { Sound knots } 1 . e . D / 3 \text { and less than } 5 \text { inches. } \\
\text { Unsound knots 1.e. D/6 and less than } 21 / 2 \text { inches. }\end{array}$ \\
\hline 4 & $6+$ & $8-16$ & 50 & 50 & No limit & No limit \\
\hline
\end{tabular}

'Plus trim.

2Disregard all knots less than $1 / 2$-inch diameter in all grades.

${ }^{3}$ The sum of the diameter of sound knots plus twice the sum of the diameter of unsound knots (in inches) is less than or equal to half of the diameter of the $\log$ (inches).

${ }^{4}$ I.e. means less than or equal to.

${ }^{5} \mathrm{D}$ means d.i.b. of $\log$ at location of knot.

\section{LOG GRADES FOR JACK PINE AND RED PINE}

Grade 1: $\operatorname{logs}$ with three or four clear faces. ${ }^{12}$

Grade 2: logs with one or two clear faces.

Grade 3: $\operatorname{logs}$ with no clear faces.

After the tentative log grade is established, the log will be degraded one grade for each of the following, except that no log can be degraded below grade 3 . Net scale after deduction for defect must be at least 50 percent of the gross contents of the log.

1. Sweep. Degrade any tentative 1 or 2 log one grade if sweep amounts to 3 or more inches and equals or exceeds one-third the diameter inside bark at small end.

2. Heart rot. Degrade any tentative 1 or $2 \log$ one grade if conk, massed hyphae, or other evidence of advanced heart rot is found anywhere in it.

\footnotetext{
${ }^{12}$ A face is one fourth of the circumference in width extending full length of the log. Clear faces are those free of: knots measuring more than 1/2-inch in diameter, overgrown knots of any size, holes more than 1/3-inch in diameter. Faces may be rotated to obtain the maximum number of clear ones.
}

\section{LOG GRADES FOR ALL O'THER SOFTWOOD LOGS}

\section{Grade 1}

1. Logs must be 16 inches in diameter or larger, 10 feet in length or longer, and have not more than 30 percent of gross scale deducted for defect.

2. Logs must be at least 75 percent clear on each of three faces.

3. All knots outside clear cutting must be sound and not more than $2 \frac{1}{2}$-inches in size.

\section{Grade 2}

1. Logs must be 12 inches in diameter or larger, 10 feet in length or longer, and have a net scale of at least 50 percent of the gross contents of the log after deduction for defect.

2. Logs must be at least 50 percent clear on each of three faces or 75 percent clear on two faces.

\section{Grade 3}

1. Logs must be 6 inches in diameter or larger, 8 feet in length or longer, and have a net scale of at least 50 percent of the gross contents of the log after deduction for defect. 
Note: (A) Diameters are diameter inside bark (d.i.b.) at small end of log.

(B) Percent clear refers to percent clear in one continuous section.

\section{METRIC EQUIVALENTS OF UNITS USED IN THIS REPORT}

1 acre $=4,046.86$ square meters or 0.405 hectare. 1,000 acres $=405$ hectares.

1 cubic foot $=0.0283$ cubic meter.

1 foot $=30.48$ centimeters or 0.3048 meter.

1 inch $=25.4$ millimeters, 2.54 centimeters, or 0.0254 meter.

1 pound $=0.454$ kilograms.

1 ton $=0.907$ metric tons

\section{TREE SPECIES GROUPS IN INDIANA $^{13}$}

\section{SOFTWOODS}

Jack pine . . . . . . . . . . . Pinus banksiana Red pine ................ Pinus resinosa White pine............... Pinus strobus Shortleaf pine ............. Pinus echinata Other yellow pine

Virginia pine .......... Pinus virginiana Tamarack . . . . . . . . . . . . . Larix laricina Baldcypress........... Taxodium distichum Eastern redcedar . . . . . . . . Juniperus virginiana Other softwoods

Scotch pine ............ Pinus sylvestris HARDWOODS

Select white oak

White oak ${ }^{14} \ldots \ldots \ldots \ldots \ldots$..... Quercus alba Swamp white oak ${ }^{14} \ldots \ldots$. Quercus bicolor Bur oak ${ }^{14} \ldots . . \ldots$.... Quercus macrocarpa Swamp chestnut oak $^{14} \ldots$. Quercus michauxii Chinkapin oak ${ }^{14} \ldots \ldots$ Quercus muehlenbergii Other white oak

Overcup oak ${ }^{14} \ldots \ldots \ldots \ldots$. . Quercus lyrata Chestnut oak ${ }^{14} \ldots \ldots \ldots \ldots$. . Quercus prinus Post oak $^{14} \ldots \ldots \ldots \ldots$. . Quercus stellata Select red oak

Cherrybark oak ${ }^{14} Q u e r c u s$ falcata var. pagodifolia Northern red oak ${ }^{14} \ldots . . . . .$. Quercus rubra Shumard oak $^{14} \ldots . . . \ldots$ Quercus shumardii

${ }^{13}$ The common and scientific names are based on: Little, Elbert L 1979. Checklist of native and naturalized trees of the United States. Agric Handb. 541. Washington, DC: U.S. Department of Agriculture, Forest Service $375 p$.

${ }^{14}$ This species is considered a hard hardwood, with an average specific gravity greater than or equal to 0.50 .
Other red oaks

Scarlet oaks ${ }^{14} \ldots \ldots \ldots \ldots$. . Quercus coccinea Northern pin oak ${ }^{14} \ldots$... Quercus ellipsoidalis Southern red oak ${ }^{14} \ldots \ldots$... Quercus falcata Shingle oak ${ }^{14} \ldots \ldots \ldots$. . Quercus imbricaria Black oak ${ }^{14} \ldots \ldots \ldots \ldots \ldots$. Quercus velutina Blackjack oak ${ }^{14} \ldots \ldots$. . Quercus marilandica Pin oak $^{14} \ldots \ldots \ldots \ldots \ldots$..... Quercus palustris Select hickory

Pecan $^{14} \ldots \ldots \ldots \ldots$. . . . . . Carya illinoensis Shellbark hickory ${ }^{14} \ldots \ldots \ldots$. Carya lacinosa Shagbark hickory ${ }^{14} \ldots \ldots \ldots \ldots$. . Carya ovata Mockernut hickory ${ }^{14} \ldots . .$. Carya tomentosa Other hickory

Bitternut hickory ${ }^{14} \ldots \ldots$. Carya cordiformis Pignut hickory ${ }^{14} \ldots \ldots \ldots \ldots$. . Carya glabra Birch

Yellow birch ${ }^{14} \ldots \ldots \ldots$ Betula alleghaniensis River birch $^{14} \ldots \ldots \ldots \ldots \ldots \ldots$. . . . . . . . nigra

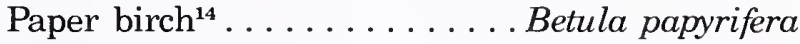
Hard maple

Black maple ${ }^{14} \ldots \ldots \ldots \ldots \ldots$. . Acer nigrum Sugar maple ${ }^{14} \ldots \ldots \ldots \ldots$. . Acer saccharum Soft maple

Red maple ${ }^{15} \ldots \ldots \ldots \ldots \ldots$. . . . Acer rubrum

Silver maple ${ }^{15} \ldots \ldots \ldots \ldots$. . Acer saccharinum Ash

Black ash ${ }^{15} \ldots \ldots \ldots \ldots \ldots$. . Fraxinus nigra Blue ash $^{14} \ldots \ldots \ldots$. . Fraxinus quadrangulata White $\operatorname{ash}^{14} \ldots \ldots \ldots \ldots$. . Fraxinus americana Green ash ${ }^{14} \ldots \ldots \ldots$. Fraxinus pennsylvanica Cottonwood $^{15} \ldots \ldots \ldots \ldots \ldots$. Populus deltoides Aspen

Bigtooth aspen ${ }^{15} \ldots \ldots$. . Populus grandidentata Quaking aspen ${ }^{15} \ldots \ldots \ldots$ Populus tremuloides Balsam poplar ${ }^{15}$. . . . . . . Populus balsamifera Basswood

American basswood ${ }^{15} \ldots . .$. . Tilia americana White basswood ${ }^{15} \ldots \ldots \ldots$ Tilia heterophylla Beech $^{14} \ldots \ldots \ldots \ldots \ldots$. . . . Fagus grandifolia Black walnut ${ }^{14} \ldots \ldots \ldots \ldots \ldots \ldots$ Juglans nigra Black cherry ${ }^{15} \ldots \ldots \ldots \ldots \ldots \ldots$ Prunus serotina Butternut ${ }^{15} \ldots \ldots \ldots \ldots \ldots \ldots$ Juglans cinerea Elm

Winged $\operatorname{elm}^{15} \ldots \ldots \ldots \ldots$. . . . . . alata American $\mathrm{elm}^{15} \ldots . . \ldots$. . Ulmus americana Siberian $\operatorname{elm}^{15} \ldots \ldots \ldots \ldots \ldots$. . . Ulmus pumila

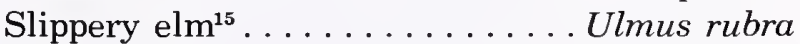
Rock $\operatorname{elm}^{14} \ldots \ldots \ldots \ldots$. . . Ulmus thomasii Hackberry ${ }^{15} \ldots \ldots \ldots \ldots \ldots$ Celtis occidentalis Sycamore $^{15} \ldots \ldots \ldots \ldots$. . . Platanus occidentalis Yellow-poplar ${ }^{15} \ldots \ldots \ldots$. . Liriodendron tulipifera

\footnotetext{
${ }^{15}$ This species is considered a soft hardwood, with an average specific gravity of 0.50 or less.
} 
Black willow ${ }^{15} \ldots \ldots \ldots \ldots$. . . Salix nigra

Sweetgum $^{15} \ldots \ldots \ldots \ldots$ Liquidambar styraciflua

Tupelo

Black tupelo ${ }^{15} \ldots$. . Nyssa sylvatica var. sylvatica

Swamp tupelo15. . . . Nyssa sylvatica var. biflora

Persimmon $^{14} \ldots \ldots \ldots \ldots$ Diospyros virginiana

Sassafras $^{15} \ldots \ldots \ldots$. . . . Sassafras albidum

Other hardwoods

Ohio buckeye ${ }^{15}$. . . . . . . . . Aesculus glabra

Boxelder $^{15}$............... Acer negundo

Kentucky coffeetree ${ }^{14}$. . . . Gymnocladus dioicus

Black locust ${ }^{14}$. . . . . . Robinia pseudoacacia

White mulberry ${ }^{15} \ldots \ldots \ldots \ldots$. . . . .

Red mulberry ${ }^{15} \ldots \ldots \ldots \ldots$................

Flowering dogwood ${ }^{14} \ldots \ldots \ldots$. Cornus florida

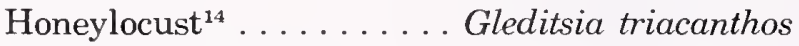

Northern catalpa ${ }^{15} \ldots \ldots$. . . Catalpa speciosa

European alder ${ }^{15} \ldots \ldots \ldots \ldots$. Alnus glutinosa

White $\operatorname{poplar}^{15} \ldots \ldots \ldots \ldots \ldots$. . . . . . .

Yellow buckeye ${ }^{15}$. . . . . . . Aesculus octandra

Noncommercial species ${ }^{16}$

Osage-orange........... Maclura pomifera

Eastern hophornbeam . . . . . . Ostrya virginiana

Apple ................. Malus spp.

American hornbeam .... Carpinus caroliniana

Wild plum ............... Prunus spp.

Eastern redbud.......... Cercis canadensis

Pawpaw . . . . . . . . . . Asimina triloba

Hawthorn ............. Crataegus spp.

Ailanthus............. Ailanthus altissima

Chokecherry........... Prunus virginiana

\section{DEFINITION OF TERMS}

\section{Average annual removals from growing stock -}

The average net growing-stock volume in growingstock trees removed annually for forest products (including roundwood products and logging residues) and for other uses (see other removals). Average annual removals of growing stock are reported for a period of several years (1966 to 1985 in this report) and are based on information obtained from remeasurement plots (see Survey Procedures).

Average annual removals from sawtimber.-The average net board foot sawtimber volume of live sawtimber trees removed annually for forest products (including roundwood products and other uses [see other removals]). Average annual removals of sawtimber are repoited for a period of several years

\footnotetext{
${ }^{16}$ Individual noncommercial species were not classified as soft or hard hardwoods since they are predominately hard hardwoods, the entire group was classed as hard.
}

(1966 to 1985 in this report) and are based on information obtained from remeasurement plots (see Survey Procedures).

Basal area.-The area in square feet of the cross section at breast height of a single tree. When the basal area of all trees in a stand are summed, the result is usually epressed as square feet of basal area per acre.

Biomass.-The above-ground volume of all live trees (including bark and foliage) reported in green tons. Biomass is made up of 7 components:

Growing-stock stumps.-Biomass of a growingstock tree 1-foot stump.

Growing-stock bole-Biomass of a growing-stock tree from a 1-foot stump to a variable 4-inch top.

Growingstock tops and limbs.-Biomass of a growing-stock tree from a 1 -foot stump minus the growing-stock bole.

Cull stumps.-Biomass of a cull tree 1-foot stump.

Cull bole.-Biomass of a cull tree from a 1-foot stump to a variable 4 -inch top.

Cull tops and limbs.-Biomass of a cull tree from a 1-foot stump minus the cull bole.

1- to 5-inch trees.-Biomass of all live trees from 1- to 5-inches in diameter at breast height.

Commercial species.-Tree species presently or prospectively suitable for industrial wood products. (Note: Excludes species of typically small size, poor form, or inferior quality such as hophornbeam, osage-orange and redbud.)

Commercial forest land.-(See timberland).

County and municipal land.-Land owned by counties and local public agencies or municipalities, or land leased to these governmental units for 50 years or more.

Cropland.-Land under cultivation within the past 24 months; including cropland harvested, crop failures, cultivated summer fallow, idle cropland used only for pasture, orchards, and land in soil improvement crops, but excluding land cultivated in developing improved pasture.

Cull--Portions of a tree that are unusable for industrial wood products because of rot, form, or other defect.

Diameter classes. - A classification of trees based on diameter outside bark, measured at breast height ( $4 \frac{1 / 2}{2}$ feet above the ground). (Note: D.b.h. is the common abbreviation for diameter at breast height. Two-inch diameter classes are commonly used in Forest Inventory and Analysis, with the even inch the approximate midpoint for a class. For example, the 6-inch class includes trees 5.0 through 6.9 inches d.b.h.)

Farm.-Any place from which $\$ 1,000$ or more of agricultural products were produced and sold during the year. 
Farmer-owned land.-Land owned by farm operators. (Note: Excludes land leased by farm operators from nonfarm owners, such as railroad companies and States.)

Forest land.-Land at least 16.7 percent stocked by forest trees of any size, or formerly having had such tree cover, and not currently developed for nonforest use. (Note: Stocking is measured by comparing specified standards with basal area and/or number of trees, age or size, and spacing.) The minimum area for classification of forest land is 1 acre. Roadside, streamside, and shelterbelt strips of timber must have a crown width of at least 120 feet to qualify as forest land. Unimproved roads and trails, streams, or other bodies of water or clearings in forest areas shall be classed as forest if less than 120 feet wide. Also see definitions for tree, land, timberland, reserved timberland, stocking, and water.

Forest industry land.-Land owned by companies or individuals operating primary wood-using plants.

Forest type.-A classification of forest land based on the species forming a plurality of live tree stocking. Major forest types in the State are:

Jack-red-white pine-Forests in which jack, red or white pine, singly or in combination, comprise a plurality of the stocking. (These species are generally found in plantations in Indiana.)

Shortleaf pine.-Forests in which shortleaf pine comprises a plurality of the stocking. (Primarily plantations in Indiana.)

Scotch-Virginia pine-Forests in which Scotch and Virginia pines and eastern redcedar, singly or in combination, comprise a plurality of the stocking. (Common associates include oak, yellowpoplar, red maple, sassafras, and white pine.)

Oak-pine.-Forests in which hardwoods (usually white, scarlet, chestnut, northern red or black oaks), singly or in combination, comprise a plurality of the stocking but where pines or eastern redcedar comprise 25 to 50 percent of the stocking. (Common associates include gum, hickory, sassafras, and yellow-poplar.)

Oak-hickory.-Forests in which upland oaks or hickories, singly or in combination, comprise a plurality of the stocking, and less than 25 percent of the stocking is in white, Scotch, and Virginia pines or eastern redcedar. (Common associates include yellow-poplar, elm, maple, black walnut, black locust, and sassafras.)

Chestnut-scarlet oak.-Forests in which chestnut oak or scarlet oak, singly or in combination, comprise a plurality of the stocking. (Common associates include eastern redcedar, black oak, white oak, and hickory.)
Sassafras-persimmon.-Forests in which sassafras and persimmon, singly or in combination, comprise at least 50 percent of the stocking. (Common associates include oak, yellow-poplar, elm, maple, and eastern redcedar.)

Oak-gum.-Bottomland forests in which tupelo, blackgum, sweetgum, oaks, or cypress, singly or in combination, comprise a plurality of the stocking. (Common associates include cottonwood, willow, ash, elm, hackberry, and maple.)

Lowland oak.-Bottomland forests in which wet site oaks such as swamp chestnut, cherrybark, and pin oak, singly or in combination, comprise a plurality of the stocking. (Common associates are swamp white oak, bur oak, soft maple, and sycamore.)

Elm-ash-soft maple--Forests in which lowland elm, ash, soft maple, and cottonwood, singly or in combination, comprise a plurality of the stocking. (Common associates include boxelder, willow, sycamore, and beech.)

Cottonwood.-Forests in which cottonwood comprises at least 50 percent of the stocking. (Associates include willow, elm, soft maple, and ash.)

Maple-beech.-Forests in which hard maple or beech, singly or in combination, comprise a plurality of the stocking. (Common associates include soft maple, elm, and basswood.)

Cherry-ash-yellow-poplar-Forests in which black cherry, white ash, and yellow-poplar, singly or in combination, comprise a plurality of the stocking. (Common associates include oak, maple, black walnut, beech, basswood, and sycamore.)

Gross area.-The entire area of land and water as determined by the Soil Conservation Service, 1980.

Growing-stock trees.-Live trees of commercial species that meet specified standards of size, quality, and merchantability. (Note: Excludes rough and rotten trees.)

Growing-stock volume.-Net volume in cubic feet of growing-stock trees 5 inches d.b.h. and over, from one foot above the ground to a minimum 4 inch top diameter outside bark of the central stem or to the point where the central stem breaks into limbs. Cubic feet can be converted to standard cords by dividing by 79 . One standard cord is 128 cubic feet of stacked wood, including bark and air.

Hard hardwoods.-Hardwood species with an average specific gravity greater than or equal to 0.50 such as oaks, hard maple, hickories, and ash.

Hardwoods.-Dicotyledonous trees, usually broadleaved and deciduous. See soft hardwoods and hard hardwoods.

Idle farmland.-Includes former cropland, orchards, improved pastures, and farm sites not tended 
within the past 2 years and presently less than 16.7 percent stocked with trees.

Improved pasture--Land currently improved for grazing by cultivating, seeding, irrigating, or clearing of trees or brush and less than 16.7 percent stocked with live trees.

Industrial wood.- All roundwood products, except fuelwood.

Land.-A. Bureau of the Census. Dry land and land temporarily or partly covered by water such as marshes, swamps, and river flood plains (omitting tidal flats below mean high tide); streams, sloughs, estuaries, and canals less than one-eighth of a statute mile wide; and lakes, reservoirs, and ponds less than 40 acres in area. This is the same definition that the Soil Conservation Service uses in the National Resource Inventory. Bureau of Census estimates of total land area where used in 1967; Soil Conservation Service estimates were used for 1986.

B. Forest Inventory and Analysis. The same as the Soil Conservation Service, except minimum width of streams, etc., is 120 feet and minimum size of lakes, etc., is 1 acre.

Live trees.-Growing-stock, rough, and rotten trees 1-inch d.b.h. and larger.

Log grades.-A classification of logs based on external characteristics as indicators of quality or value. (See Appendix for specific grading factors used.)

Logging residues.-The unused growing stock portions of trees cut or killed by logging.

Maintained road-Any road, hard-topped or other surfaces, that is plowed or graded at least once a year. Includes rights-of-way that are cut or treated to limit herbaceous growth.

Marsh.-Nonforest land that characteristically supports low, generally herbaceous or shrubby vegetation and that is intermittently covered with water.

Merchantable.-Refers to a pulpwood or saw log section that meets pulpwood or saw log specifications, respectively.

Miscellaneous federal land.-Federal land other than National Forest.

Miscellaneous private land.-Privately owned land other than forest-industry and farmer-owned land.

Mortality.-The volume of sound wood in growingstock and sawtimber trees that die annually.

National forest land.-Federal land that has been legally designated as National Forest or purchase units, and other land administered by the USDA Forest Service.

Net annual growth of growing stock.-The annual change in volume of sound wood in live sawtimber and poletimber trees and the total volume of trees entering these classes through ingrowth, less volume losses resulting from natural causes.
Net annual growth of sawtimber-The annual change in the volume of live sawtimber trees and the total volume of trees reaching sawtimber size, less volume losses resulting from natural causes.

Net volume.-Gross volume less deductions for rot, sweep, or other defect affecting use for timber products.

Noncommercial species.-Tree species of typically small size, poor form, or inferior quality that normally do not develop into trees suitable for industrial wood products.

Nonforest land.-Land that has never supported forests, and land formerly forested where use for timber management is precluded by development for other uses. (Note: Includes areas used for crops, improved pasture, residential areas, city parks, improved roads of any width and adjoining clearings, powerline clearings of any width, and 1- to 40-acre areas of water classified by the Bureau of the Census as land. If intermingled in forest areas, unimproved roads and nonforest strips must be more than 120 feet wide and more than 1 acre in area to qualify as nonforest land.)

a. Nonforest land without trees.-Nonforest land with no live trees present.

b. Nonforest land with trees.-Nonforest land with one or more trees per acre at least 5 inches d.b.h.

Nonstocked land.-Timberland less than 16.7 percent stocked with growing-stock trees.

Other removals.-Growing-stock trees removed but not utilized for products, or trees left standing but "removed" from the timberland classification by land use change. Examples are removals from cultural operations such as timber stand improvement work, land clearing, and changes in land use.

Ownership.-Property owned by one owner, regardless of the number of parcels in a specified area.

Ownership size class.-The amount of timberland owned by one owner, regardless of the number of parcels.

Owner tenure--The length of time a property has been held by the owner.

Pasture-Land presently used for grazing or under cultivation to develop grazing.

Pastured timberland.-Timberland for which the primary use is wood production, but is presently used for grazing.

Physiographic class.-A measure of soil and water conditions that affect tree growth on a site. The physiographic classes are:

Xeric sites.-Very dry soils where excessive drainage seriously limits both growth and species occurrence. Example: cedar barrens.

Xeromesic sites.-Moderately dry soils where excessive drainage limits growth and species occur- 
rence to some extent. Example: dry oak ridge.

Mesic sites-Deep, well-drained soils. Growth and species occurrence are limited only by climate.

Hydromesic sites.-Moderately wet soils where insufficient drainage or infrequent flooding limits growth and species occurrence to some extent. Example: better drained bottomland hardwood sites.

Hydric sites.-Very wet sites where excess water seriously limits both growth and species occurrence. Example: frequently flooded river bottoms and cypress swamps.

Plant byproducts.-Plant residues used for products such as mulch, pulp chips, and fuelwood.

Plant residues.-Wood and bark materials generated at manufacturing plants during production of other products.

Poletimber stands.-(See stand-size class.)

Poletimber trees.-Growing-stock trees of commercial species at least 5 inches d.b.h. but smaller than sawtimber size.

Reserved timberland.-Forest land sufficiently productive to qualify as timberland but withdrawn from timber utilization through statute, administrative regulation, designation, or exclusive use for Christmas tree production, as indicated by annual shearing. Formerly called productivereserved forest land.

Rotten trees.-Live trees of commercial species that do not contain at least one 12 -foot saw log or two saw logs 8 feet or longer, now or prospectively, and/or do not meet regional specifications for freedom from defect primarily because of rot; that is, when more than 50 percent of the cull volume in a tree is rotten.

Rough trees.-(a) Live trees of commercial species that do not contain at least one merchantable 12 -foot saw $\log$ or two saw $\operatorname{logs} 8$ feet or longer, now or prospectively, and/or do not meet regional specifications for freedom from defect primarily because of roughness or poor form, and (b) all live trees of noncommercial species.

Roundwood products.-Logs, bolts, or other round sections (including chips from roundwood) cut from trees for industrial or consumer uses. (Note: Includes saw logs, veneer logs, and bolts; cooperage logs and bolts; pulpwood; fuelwood; piling; poles; posts; hewn ties; mine timbers; and various other round, split, or hewn products.)

Salvable dead trees.-Standing or down dead trees considered merchantable by regional standards. Saplings.-Live trees 1 to 5 inches d.b.h.

Sapling-seedling stands.-(See stand-size class.)

Saw log.-A log meeting minimum standards of diameter, length, and defect, including logs at least 8 feet long, sound and straight and with a minimum diameter outside bark (d.o.b.) for softwoods of 7 inches ( 9 inches for hardwoods) or other combinations of size and defect specified by regional standards.

Saw log portion.-That part of the bole of sawtimber trees between the stump and the saw log top.

Saw log top.-The point on the bole of sawtimber trees above which a saw log cannot be produced. The minimum saw log top is 7 inches d.o.b. for softwoods and 9 inches d.o.b. for hardwoods.

Sawtimber stands.-(See stand-size class.)

Sawtimber trees.-Growing-stock trees of commercial species containing at least a 12 -foot saw log or two noncontiguous saw logs 8 feet or longer, and meeting regional specifications for freedom from defect. Softwoods must be at least 9 inches d.b.h. Hardwoods must be at least 11 inches d.b.h.

Sawtimber volume--Net volume of the saw log portion of live sawtimber in board feet, International $1 / 2$-inch rule (unless specified otherwise) from stump to a minimum 7 inches top diameter outside bark (d.o.b.) for softwoods and a minimum 9 inches top d.o.b. for hardwoods.

Seedlings.-Live trees less than 1 inch d.b.h. that are expected to survive. Only softwood seedlings more than 6 inches tall and hardwood seedlings more than 1 foot tall are counted.

Short-log (rough tree).-Sawtimber-size trees of commercial species that contain at least one merchantable 8- to 11 -foot saw log but not a 12 -foot saw log.

Site class.-A classification of forest land in terms of inherent capacity to grow crops of industrial wood based on fully stocked natural stands.

Site index.-An expression of forest site quality based on the height of a free-growing dominant or codominant tree of a representative species in the forest type at age 50 .

Soft hardwoods.-Hardwood species with an average specific gravity less than 0.50 such as gum, yellow-poplar, cottonwood, red maple, basswood, and willow.

Softwoods.-Coniferous trees, usually evergreen, having needles or scale-like leaves.

Stand.-A group of trees on a minimum of 1 acre of forest land that is stocked by forest trees of any size.

Stand-age class.-Age of the main stand. Main stand refers to trees of the dominant forest type and stand-size class.

Stand-area class.-The extent of a continuous forested area of the same forest type, stand-size class, and stand-density class.

Stand-size class.-A classification of stocked (see stocking) forest land based on the size class of live trees on the area; that is, sawtimber, poletimber, or seedlings and saplings. 
a. Sawtimber stands.-Stands with half or more of live stocking in sawtimber or poletimber trees, and with sawtimber stocking at least equal to poletimber stocking.

b. Poletimber stands.-Stands with half or more live stocking in poletimber and/or sawtimber trees, and with poletimber stocking exceeding that of sawtimber.

c. Sapling-seedling stands.-Stands with more than half of the live stocking in saplings and/or seedlings.

State land.-Land owned either by States or leased to them, for 50 years or more.

Stocking.-The degree of occupancy of land by trees, measured by basal area and/or the number of trees in a stand by size or age and spacing, compared to the basal area and/or number of trees required to fully utilize the growth potential of the land; that is, the stocking standard.

A stocking percent of 100 indicates full utilization of the site and is equivalent to 80 square feet of basal area per acre in trees 5 inches d.b.h. and larger. In a stand of trees less than 5 inches d.b.h., a stocking percent of 100 would indicate that the present number of trees is sufficient to produce 80 square feet of basal area per acre when the trees reach 5 inches d.b.h.

Stands are grouped into the following stocking classes:

Overstocked stands.-Stands in which stocking of trees is 130.0 percent or more.

Fully stocked stands.-Stands in which stocking of trees is from 100.0 to 129.9 percent.

Medium stocked stands.-Stands in which stocking of trees is from 60.0 to 99.9 percent.

Poorly stocked stands.-Stands in which stocking of trees is from 16.7 to 59.9 percent.

Nonstocked areas.-Commercial forest land on which stocking of trees is less than 16.7 percent.

Timberland.-Forest land producing or capable of producing crops of industrial wood and not withdrawn from timber utilization. (Note: Areas qualifying as timberland are capable of producing more than 20 cubic feet per acre per year of annual growth when managed. Currently inaccessible and inoperable areas are included except when the areas involved are small and unlikely to become suitable for producing industrial wood in the foreseeable future.) Formerly called commercial forest land. Also see definition of pastured timberland.

Timber removals from growing stock.-The net volume of growing stock in growing-stock trees removed for forest products (including roundwood products and logging residues) and for other uses (see other removals). Timber removals from grow- ing stock are reported for a single year (1985 in this report) and are based on information obtained from a survey of primary wood-using mills (see Survey Procedures).

Timber removals from sawtimber--The net boardfoot volume of live sawtimber trees removed for forest products (including roundwood products and logging residues) and for other uses (see other removals). Timber removals from sawtimber are reported for a single year (1985 in this report) and are based on information obtained from a survey of primary wood-using mills (see Survey Procedures).

Timber products output.-All timber products cut from roundwood and byproducts of wood manufacturing plants. Roundwood products include logs, bolts, or other round sections cut from growingstock trees, cull trees, salvable dead trees, trees on nonforest land, noncommercial species, sapling-size trees, and limbwood. Byproducts from primary manufacturing plants include slabs, edging, trimmings, miscuts, sawdust, shavings, veneer cores and clippings, and screenings of pulpmills that are used as pulpwood chips or other products.

Trees.-Woody plant having a well-developed stem and usually more than 12 feet tall at maturity.

Tree biomass.-The total aboveground weight (including the bark) of all trees from 1 to 5 inches in d.b.h., and the total aboveground weight (including the bark) from a 1-foot stump for trees more than 5 inches in diameter.

Tree size class.-A classification of trees based on diameter at breast height, including sawtimber trees, poletimber trees, saplings, and seedlings.

Upper stem portion.-That part of the bole of sawtimber trees above the saw $\log$ top to a minimum top diameter of 4 inches outside bark or to the point where the central stem breaks into limbs.

Urban and other areas.-Areas within the legal boundaries of cities and towns; suburban areas developed for residential, industrial, or recreational purposes; schoolyards; cemeteries; roads; railroads; airports; beaches; powerlines and other rights-ofway; or other nonforest land not included in any other specified land use class.

Urban forest land.-Land that would otherwise meet the criteria for timberland, but is in an urban-suburban area surrounded by commercial, industrial, or residential development.

Water-(a) Bureau of the Census.-Permanent inland water surfaces, such as lakes, reservoirs, and ponds at least 40 acres in area; and streams, sloughs, estuaries, and canals at least one-eighth of a statute mile wide. This is the same definition that the Soil Conservation Service uses in the National 
Resource Inventory. Bureau of Census estimates of total water area were used in 1967; Soil Conservation Service estimates were used for 1986.

(b) Noncensus.-Permanent inland water surfaces, such as lakes, reservoirs, and ponds from 1 to 39.9 acres in area; and streams, sloughs, estuaries, and canals from 120 feet to one-eighth of a statute mile wide.

Windbreaks.-A group of trees whose primary use is to protect buildings currently in use.

Wooded pasture.-Improved pasture with more than 16.7 percent stocking in live trees but less than 25 percent stocking in growing-stock trees. Area is currently improved for grazing or there is other evidence of grazing.

Wooded strip.-An acre or more of natural continuous forest land that would otherwise meet survey standards for timberland except that it is less than 120 feet wide.

Woodland.-Forest land incapable of producing 20 cubic feet per acre of annual growth or of yielding crops of industrial wood under natural conditions because of adverse site conditions. (Note: Adverse conditions include shallow soil, dry climate, poor drainage, high elevation, steepness, and rockiness.) Formerly called unproductive forest land.

\section{TABLES}

\section{Text Tables}

Table 1.-Dates of aerial photography by county and Forest Survey Unit

Table 2.-Aerial photo points classified by photo land class and Forest Survey Unit

Table 3.-Number of ground plots by ground land use class and Forest Survey Unit

Table 4.-Factors to convert net cubic foot volume in growing-stock trees to board feet by diameter class and softwoods and hardwoods.

Table 5.-Factors to determine saw log volume as a proportion of growing-stock bole volume by diameter class and softwoods and hardwoods

\section{Area}

Table 6.-Area of land by land class and Forest Survey Unit, 1950, 1967, 1986

*Indicates core table common to all eastern FIA statistical reports.
Table 7.-Area of timberland by county, 1950, 1967, and 1986

Table 8.-Area of timberland by stand-size class, 1950, 1967, and 1986

Table 9.-Area of land by land use class and Forest Survey Unit

*Table 10.-Area of land by county and major land use class

*Table 11.-Area of timberland by county and ownership class

*Table 12.-Area of timberland by county and forest type

*Table 13.-Area of timberland by county and standsize class

*Table 14.-Area of timberland by county and site class

*Table 15.-Area of timberland by county and stocking class of growing-stock trees

*Table 16.-Area of timberland by forest type, ownership class, and Forest Survey Unit

Table 17.-Area of timberland by ownership class, site class, and Forest Survey Unit

Table 18.-Area of privately owned timberland by ownership class, owner tenure, and size of holding

*Table 19.-Area of timberland by ownership class, stocking class of growing-stock trees, and Forest Survey Unit

*Table 20.-Area of timberland by forest type, standsize class, and Forest Survey Unit

Table 21.-Area of timberland by ownership class, stand-volume class, and Forest Survey Unit

Table 22.-Area of timberland by forest type, standsize class, and ownership class

Table 23.-Area of timberland by forest type, standsize class, and site class

Table 24.-Area of timberland by forest type, standage class, and Forest Survey Unit

Table 25.-Area of timberland by forest type, siteindex class, and Forest Survey Unit

Table 26.-Area of timberland by forest type, standsize class, and basal-area class

Table 27.-Area of timberland by stocking class based on selected stand components and Forest Survey Unit

Table 28.-Area of timberland in plantations by forest type, stand-age class, and Forest Survey Unit

Table 29.-Area of reserved timberland by ownership class and Forest Survey Unit

Table 30.-Area of reserved timberland by forest type and Forest Survey Unit

Table 31.-Area of nonforest land with trees by forest type, land use, and Forest Survey Unit 


\section{Number of Trees}

*Table 32.-Number of all live trees on timberland by species group and diameter class

*Table 33.-Number of growing-stock trees on timberland by species group and diameter class

\section{Volume}

Table 34.-Net volume of growing stock on timberland by species and Forest Survey Unit, 1967 and 1986

Table 35.-Net volume of sawtimber on timberland by species and Forest Survey Unit, 1967 and 1986

Table 36.-Net volume of all live trees on timberland by species group and diameter class

*Table 37.-Net volume of timber on timberland by class of timber and major species group

*Table 38.-Net volume of growing stock in the saw log portion of sawtimber trees on timberland by species group and diameter class

Table 39.-Net volume of growing-stock, sawtimber, short-log, and rough and rotten trees on timberland by individual species (commercial species only)

Table 40.-Net volume of noncommercial species on timberland by individual species

*Table 41.-Net volume of growing stock on timberland by species group and diameter class

*Table 42.-Net volume of sawtimber on timberland by species group and diameter class

*Table 43.-Net volume of live trees and growing stock on timberland by ownership class and major species group

Table 44.-Net volume of growing stock on timberland by species group and forest type

Table 45.-Net volume of sawtimber on timberland by species group and forest type

Table 46.-Net volume of growing stock on timberland by species group and ownership class

Table 47.-Net volume of sawtimber on timberland by species group and ownership class

Table 48.-Net volume of growing stock on timberland by forest type, stand-age class, and Forest Survey Unit

Table 49.-Net volume of sawtimber on timberland by forest type, stand-age class, and Forest Survey Unit
Table 50.-Net volume of growing stock on timberland by forest type, stand-size class, and basal-area class

Table 51.-Net volume of sawtimber on timberland by forest type, stand-size class, and basalarea class

*Table 52.-Net volume of growing stock and sawtimber on timberland by county and major species group

*Table 53.-Net volume of sawtimber on timberland by species group and tree grade

Table 54.-Net volume of sawtimber on timberland, by species group, log grade, and diameter class

Table 55.-Net volume of short-log trees on timberland by species group and diameter class (cubic feet)

Table 56.-Net volume of short-log trees on timberland by species group and diameter class (board feet)

\section{Growth}

Table 57.-Net annual growth of growing stock and sawtimber on timberland by softwoods and hardwoods, 1966 and 1985

Table 58.-Net annual growth of growing stock on timberland by species group and ownership class

Table 59.-Net annual growth of sawtimber on timberland by species group and ownership class

Table 60.-Net annual growth of growing stock on timberland by species group and forest type

Table 61.-Net annual growth of sawtimber on timberland by species group and forest type

Table 62.-Net annual growth of growing stock on timberland by forest type, stand-age class, and Forest Survey Unit

Table 63.-Net annual growth of sawtimber on timberland by forest type, stand-age class, and Forest Survey Unit

Table 64.-Net annual growth of growing stock on timberland by forest type, stand-size class, and basal area class

Table 65.-Net annual growth of sawtimber on timberland by forest type, stand-size class, and basal area class

*Table 66.-Net annual growth of growing stock and sawtimber on timberland by county and major species group 


\section{Removals}

Table 67.-Timber removals from growing stock and sawtimber on timberland by species group, 1966 and 1985

*Table 68.-Average annual removals of growing stock and sawtimber on timberland by county and major species group, 1966-1985

Table 69.-Average annual removals of growing stock on timberland by species group and Forest Survey Unit, 1966-1985

Table 70.-Average annual removals of sawtimber on timberland by species group and Forest Survey Unit, 1966-1985

Table 71.-Timber removals from growing stock and sawtimber on timberland by item and species category

*Table 72.-Net annual growth and removals of growing stock on timberland by species group and Forest Survey Unit

*Table 73.-Net annual growth and removals from sawtimber on timberland by species group and Forest Survey Unit

*Table 74.-Net annual growth and removals of growing stock on timberland by ownership class and major species group

*Table 75.-Net annual growth and removals of sawtimber on timberland by ownership class and major species group

\section{Mortality}

Table 76.-Annual mortality of growing stock on timberland by softwoods and hardwoods, 1966 and 1985

*Table 77.-Annual mortality of growing stock and sawtimber on timberland by species group

Table 78.-Annual mortality of growing stock on timberland by species group and cause of death

Table 79.-Annual mortality of sawtimber on timberland by species group and cause of death

Table 80.-Annual mortality of growing stock and sawtimber on timberland by ownership class and softwoods and hardwoods

\section{Utilization}

Table 81.-Output of timber products by product, softwoods and hardwoods, and source of material

Table 82.-Output of roundwood products by product, softwoods and hardwoods, and source of material

Table 83.-Timber products from roundwood by species group and product

Table 84.- Volume of primary plant residue by use and type of residue

\section{Biomass}

Table 85.-All live tree biomass yields on timberland by species group and forest type

Table 86.-All live tree biomass on timberland by species group and forest type

Table 87.-All live tree biomass on timberland by species group and tree biomass component (green tons)

Table 88.-All live tree biomass on timberland by species group and tree biomass component (cubic feet)

Table 89.-All live shrub biomass yields on timberland by shrub species group and forest type

\section{Sampling Error}

Table 90.-Sampling errors for Survey Unit and county totals of volume, net growth, average annual removals, and area of timberland

\section{Special Use}

Table 91.-Net volume of sawtimber on timberland by species group and diameter class (Doyle rule) 


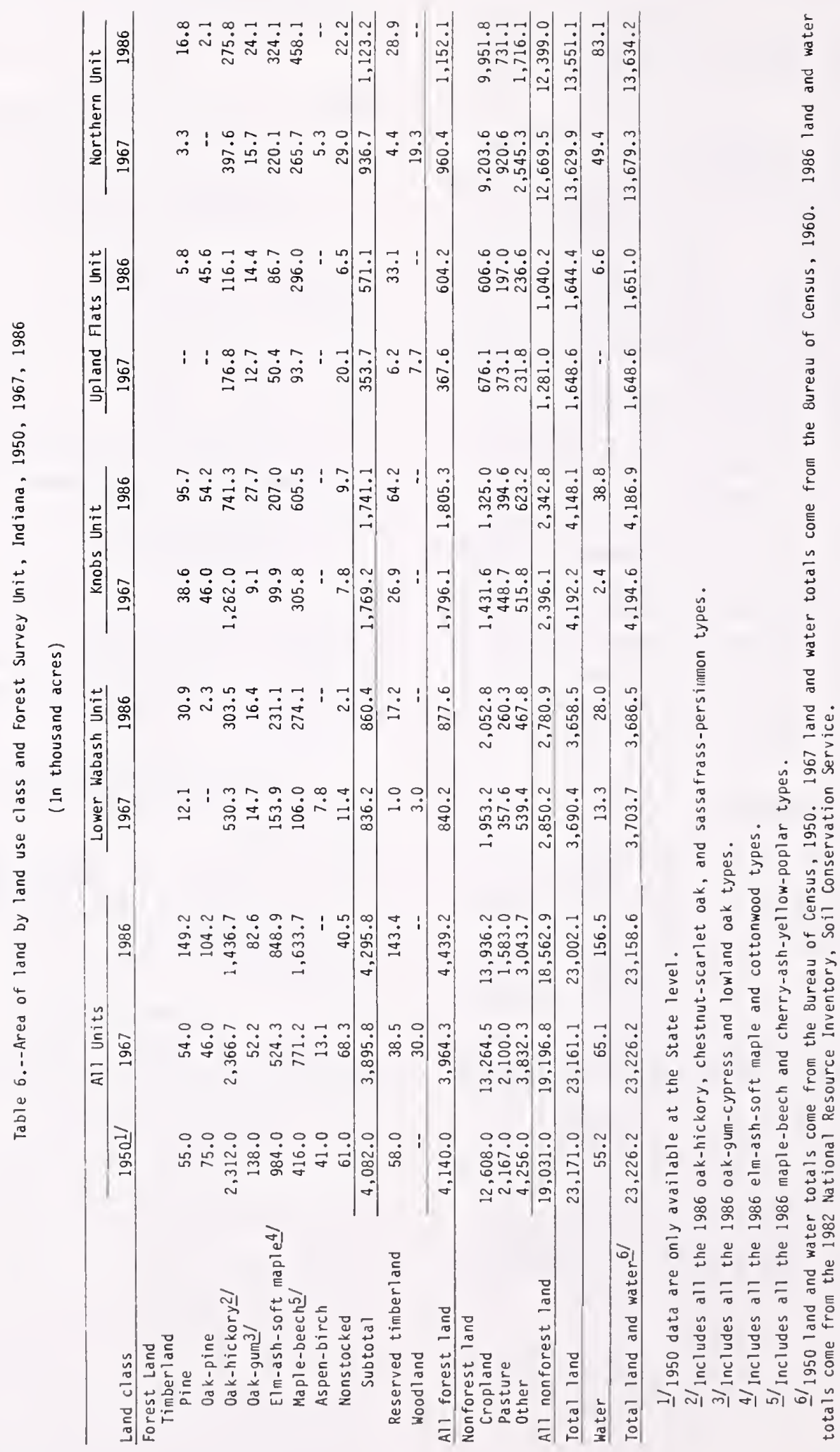


Table 7.--Area of timberland by county, Indiana, 1950,1967 , and 1986

(In thousand acres)

\begin{tabular}{|c|c|c|c|}
\hline Unit and county & 1950 & 1967 & 1986 \\
\hline $\begin{array}{l}\text { Lower Wabash Unit } \\
\text { Clay } \\
\text { Daviess } \\
\text { Gibson } \\
\text { Greene } \\
\text { Knox } \\
\text { Martin } \\
\text { Parke } \\
\text { Pike } \\
\text { Posey } \\
\text { Putnam } \\
\text { Sullivari } \\
\text { Vanderburgh } \\
\text { Vermillion } \\
\text { Vigo }\end{array}$ & $\begin{array}{r}54.0 \\
41.0 \\
43.0 \\
101.0 \\
34.0 \\
126.0 \\
97.0 \\
43.0 \\
38.0 \\
74.0 \\
49.0 \\
19.0 \\
31.0 \\
45.0 \\
\end{array}$ & $\begin{array}{r}53.0 \\
41.9 \\
48.9 \\
99.5 \\
39.4 \\
111.1 \\
90.0 \\
79.8 \\
42.1 \\
71.5 \\
53.8 \\
20.0 \\
35.0 \\
50.2 \\
\end{array}$ & $\begin{array}{r}44.3 \\
41.6 \\
42.0 \\
105.3 \\
30.8 \\
128.4 \\
87.6 \\
83.9 \\
45.1 \\
76.5 \\
64.7 \\
24.7 \\
34.7 \\
50.8 \\
\end{array}$ \\
\hline Total & 795.0 & 836.2 & 860.4 \\
\hline $\begin{array}{l}\text { Knobs Unit } \\
\text { Brown } \\
\text { Clark } \\
\text { Crawford } \\
\text { Dubois } \\
\text { Floyd } \\
\text { Harrison } \\
\text { Jackson } \\
\text { Lawrence } \\
\text { Monroe } \\
\text { Morgan } \\
\text { Orange } \\
\text { Owen } \\
\text { Perry } \\
\text { Scott } \\
\text { Spencer } \\
\text { Warrick } \\
\text { Washington }\end{array}$ & $\begin{array}{r}134.0 \\
82.0 \\
102.0 \\
86.0 \\
36.0 \\
122.0 \\
121.0 \\
125.0 \\
132.0 \\
84.0 \\
123.0 \\
114.0 \\
161.0 \\
43.0 \\
45.0 \\
49.0 \\
118.0\end{array}$ & $\begin{array}{r}133.3 \\
92.1 \\
112.8 \\
96.7 \\
36.6 \\
130.9 \\
121.9 \\
125.5 \\
133.8 \\
91.9 \\
118.4 \\
113.8 \\
142.8 \\
45.1 \\
67.8 \\
75.5 \\
130.3\end{array}$ & $\begin{array}{r}131.6 \\
84.9 \\
119.3 \\
93.0 \\
34.5 \\
132.1 \\
120.6 \\
125.3 \\
117.5 \\
86.7 \\
129.1 \\
107.5 \\
152.5 \\
43.0 \\
62.5 \\
81.4 \\
119.6 \\
\end{array}$ \\
\hline Total & $1,677.0$ & $1,769.2$ & $1,741.1$ \\
\hline $\begin{array}{l}\text { Upland Flats Unit } \\
\text { Dearborn } \\
\text { Fayette } \\
\text { Frankl in } \\
\text { Jefferson } \\
\text { Jennings } \\
\text { Ohio } \\
\text { Ripley } \\
\text { Switzerland } \\
\text { Union }\end{array}$ & $\begin{array}{l}61.0 \\
19.0 \\
70.0 \\
79.0 \\
74.0 \\
20.0 \\
61.0 \\
45.0 \\
14.0 \\
\end{array}$ & $\begin{array}{l}39.0 \\
15.8 \\
56.6 \\
60.7 \\
67.5 \\
13.2 \\
49.8 \\
38.8 \\
12.3\end{array}$ & $\begin{array}{l}91.1 \\
33.5 \\
80.4 \\
78.3 \\
87.6 \\
27.9 \\
75.7 \\
75.2 \\
21.4\end{array}$ \\
\hline Total & 443.0 & 353.7 & 571.1 \\
\hline
\end{tabular}


(Table 7 continued)

\begin{tabular}{|c|c|c|c|}
\hline Unit and county & 1950 & 1967 & 1986 \\
\hline $\begin{array}{l}\text { Northern Unit } \\
\text { Adams } \\
\text { Allen } \\
\text { Bartholomew } \\
\text { 8enton } \\
\text { 8lackford } \\
\text { Boone } \\
\text { Carroll } \\
\text { Cass } \\
\text { Clinton } \\
\text { Oecatur } \\
\text { Oe Kalb } \\
\text { Oelaware } \\
\text { Elkhart } \\
\text { Fountain } \\
\text { Fulton } \\
\text { Grant } \\
\text { Hamilton } \\
\text { Hancock } \\
\text { Hendricks } \\
\text { Henry } \\
\text { Howard } \\
\text { Huntington } \\
\text { Jasper } \\
\text { Jay } \\
\text { Johnson } \\
\text { Kosciusko } \\
\text { La Grange } \\
\text { Lake } \\
\text { La Porte } \\
\text { Madison } \\
\text { Marion } \\
\text { Marshall } \\
\text { Miami } \\
\text { Montgomery } \\
\text { Newton } \\
\text { Noble } \\
\text { Porter } \\
\text { Pulaski } \\
\text { Randol ph } \\
\text { Rush } \\
\text { St. Joseph } \\
\text { Shelby } \\
\text { Starke } \\
\text { Steuben } \\
\text { Tippecanoe } \\
\text { Tipton } \\
\text { Wabash } \\
\text { Warren } \\
\text { Wayne } \\
\text { Wells } \\
\text { White } \\
\text { Whitley }\end{array}$ & $\begin{array}{r}20.0 \\
43.0 \\
35.0 \\
2.0 \\
8.0 \\
15.0 \\
16.0 \\
29.0 \\
11.0 \\
22.0 \\
29.0 \\
17.0 \\
28.0 \\
28.0 \\
17.0 \\
18.0 \\
21.0 \\
15.0 \\
17.0 \\
19.0 \\
11.0 \\
23.0 \\
48.0 \\
17.0 \\
17.0 \\
39.0 \\
27.0 \\
30.0 \\
35.0 \\
15.0 \\
22.0 \\
28.0 \\
24.0 \\
23.0 \\
27.0 \\
28.0 \\
31.0 \\
29.0 \\
20.0 \\
11.0 \\
28.0 \\
16.0 \\
34.0 \\
20.0 \\
21.0 \\
10.0 \\
25.0 \\
19.0 \\
23.0 \\
16.0 \\
16.0 \\
28.0\end{array}$ & $\begin{array}{r}14.0 \\
35.3 \\
35.2 \\
3.0 \\
6.9 \\
11.1 \\
16.0 \\
20.2 \\
9.5 \\
23.0 \\
21.7 \\
11.2 \\
25.4 \\
26.5 \\
15.8 \\
14.0 \\
13.6 \\
8.9 \\
15.3 \\
14.2 \\
6.6 \\
20.3 \\
24.4 \\
18.6 \\
12.2 \\
27.5 \\
19.0 \\
9.5 \\
28.0 \\
11.7 \\
13.4 \\
25.1 \\
20.2 \\
21.8 \\
18.4 \\
23.2 \\
25.6 \\
24.2 \\
14.1 \\
12.1 \\
12.3 \\
21.1 \\
20.4 \\
13.0 \\
24.4 \\
22.6 \\
18.8\end{array}$ & $\begin{array}{r}14.1 \\
29.8 \\
44.8 \\
1.5 \\
9.4 \\
15.2 \\
18.4 \\
24.2 \\
9.2 \\
24.1 \\
27.3 \\
15.8 \\
29.1 \\
36.6 \\
20.4 \\
18.2 \\
20.0 \\
11.8 \\
17.7 \\
20.6 \\
8.6 \\
22.9 \\
27.0 \\
24.0 \\
20.1 \\
33.0 \\
35.9 \\
17.8 \\
41.4 \\
13.0 \\
0.9 \\
31.0 \\
25.4 \\
24.0 \\
17.9 \\
32.3 \\
30.6 \\
27.8 \\
31.9 \\
16.2 \\
13.0 \\
20.5 \\
19.7 \\
12.4 \\
22.5 \\
12.5 \\
26.9 \\
31.5 \\
22.6\end{array}$ \\
\hline Total & $1,171.0$ & 936.7 & $1,123.2$ \\
\hline All counties & $4,086.0$ & $3,895.8$ & $4,295.8$ \\
\hline
\end{tabular}


Table 8.--Area of timberland by stand-size class and Forest Survey Unit, Indiana, 1950, 1967, and 1986

( In thousand acres)

\begin{tabular}{|c|c|c|c|}
\hline Unit and stand-size class & $1950^{1 /}$ & 1967 & 1986 \\
\hline $\begin{array}{l}\text { All Units } \\
\text { Sawtimber } \\
\text { Poletimber } \\
\text { Seedling-sapling } \\
\text { Nonstocked }\end{array}$ & $\begin{array}{r}2,084.0 \\
1,337.0 \\
600.0 \\
61.0 \\
\end{array}$ & $\begin{array}{r}2,036.5 \\
865.7 \\
925.3 \\
68.3 \\
\end{array}$ & $\begin{array}{r}2,770.0 \\
673.5 \\
811.8 \\
40.5\end{array}$ \\
\hline Total & $4,082.0$ & $3,895.8$ & $4,295.8$ \\
\hline $\begin{array}{l}\text { Lower Wabash Unit } \\
\text { Sawtimber } \\
\text { Poletimber } \\
\text { Seedling-sapling } \\
\text { Nonstocked }\end{array}$ & $\begin{array}{l}-- \\
-- \\
-- \\
--\end{array}$ & $\begin{array}{r}432.2 \\
177.6 \\
215.0 \\
11.4\end{array}$ & $\begin{array}{r}582.5 \\
112.0 \\
163.8 \\
2.1\end{array}$ \\
\hline Total & -- & 836.2 & 860.4 \\
\hline $\begin{array}{l}\text { Knobs Unit } \\
\text { Sawtimber } \\
\text { Poletimber } \\
\text { Seedling-sapling } \\
\text { Nonstocked }\end{array}$ & $\begin{array}{l}-- \\
-- \\
-- \\
-\end{array}$ & $\begin{array}{r}900.8 \\
425.1 \\
435.5 \\
7.8 \\
\end{array}$ & $\begin{array}{r}1,148.7 \\
254.0 \\
328.7 \\
9.7 \\
\end{array}$ \\
\hline Total & -- & $1,769.2$ & $1,741.1$ \\
\hline $\begin{array}{l}\text { Upland Flats Unit } \\
\text { Sawtimber } \\
\text { Poletimber } \\
\text { Seedling-sapling } \\
\text { Nonstocked }\end{array}$ & $\begin{array}{l}-- \\
-- \\
-- \\
\end{array}$ & $\begin{array}{r}172.4 \\
75.8 \\
85.4 \\
20.1 \\
\end{array}$ & $\begin{array}{r}285.4 \\
113.9 \\
165.3 \\
6.5 \\
\end{array}$ \\
\hline Total & -- & 353.7 & 571.1 \\
\hline $\begin{array}{l}\text { Northern Unit } \\
\text { Sawtimber } \\
\text { Poletimber } \\
\text { Seedling-sapling } \\
\text { Nonstocked }\end{array}$ & $\begin{array}{l}-- \\
-- \\
-- \\
--\end{array}$ & $\begin{array}{r}b 31.1 \\
187.2 \\
189.4 \\
29.0\end{array}$ & $\begin{array}{r}753.4 \\
193.6 \\
154.0 \\
22.2 \\
\end{array}$ \\
\hline Total & - & 936.7 & $1,123.2$ \\
\hline
\end{tabular}

1/1950 data are only available at the State level.

Table 9.--Area of land by land use class and Forest Survey Unit, Indiana, 1986

(In thousand acres)

\begin{tabular}{|c|c|c|c|c|c|}
\hline \multirow[b]{2}{*}{ Land use class } & \multirow[b]{2}{*}{$\begin{array}{c}\text { Ali } \\
\text { Units }\end{array}$} & \multicolumn{4}{|c|}{ Forest Survey Unit } \\
\hline & & $\begin{array}{l}\text { Lower } \\
\text { Wabash }\end{array}$ & Knobs & $\begin{array}{l}\text { Upland } \\
\text { Flats }\end{array}$ & Northern \\
\hline $\begin{array}{l}\text { Forest land } \\
\text { Timberland } \\
\text { Reserved timberland }\end{array}$ & $\begin{array}{r}4,295.8 \\
143.4 \\
\end{array}$ & $\begin{array}{r}860.4 \\
17.2 \\
\end{array}$ & $\begin{array}{r}1,741.1 \\
64.2 \\
\end{array}$ & $\begin{array}{r}571.1 \\
33.1 \\
\end{array}$ & $\begin{array}{r}1,123.2 \\
28.9 \\
\end{array}$ \\
\hline Total & $4,439.2$ & 877.6 & $1,805.3$ & 604.2 & $1,152.1$ \\
\hline $\begin{array}{l}\text { Nonforest land } \\
\text { Nonforest with trees } \\
\text { Cropland with trees } \\
\text { Improved pasture with trees } \\
\text { Wooded strips } \\
\text { Idle farmland with trees } \\
\text { Marsh with trees } \\
\text { Urban timberland } \\
\text { Urban and other with trees } \\
\text { Windbreaks } \\
\text { Wooded pasture }\end{array}$ & $\begin{array}{r}78.1 \\
149.9 \\
111.7 \\
24.4 \\
12.1 \\
117.0 \\
274.6 \\
40.2 \\
120.0 \\
\end{array}$ & $\begin{array}{r}22.9 \\
33.3 \\
31.3 \\
4.1 \\
-- \\
6.5 \\
47.0 \\
12.1 \\
24.4 \\
\end{array}$ & $\begin{array}{r}19.6 \\
52.2 \\
28.0 \\
13.8 \\
1.9 \\
16.2 \\
61.4 \\
15.2 \\
35.0 \\
\end{array}$ & $\begin{array}{r}10.1 \\
28.8 \\
14.3 \\
2.1 \\
-- \\
4.9 \\
27.0 \\
1.7 \\
29.4 \\
\end{array}$ & $\begin{array}{r}25.5 \\
35.6 \\
38.1 \\
4.4 \\
10.2 \\
89.4 \\
139.2 \\
11.2 \\
31.2\end{array}$ \\
\hline Subtotal & 928.0 & 181.6 & 243.3 & 118.3 & 384.8 \\
\hline $\begin{array}{l}\text { Nonforest without trees } \\
\text { Cropland without trees } \\
\text { lmproved pasture without trees } \\
\text { Idle farm without trees } \\
\text { Marsh without trees } \\
\text { Other farm-farmstead } \\
\text { Urban and other } \\
\text { Noncensus water }\end{array}$ & $\begin{array}{r}13,858.1 \\
1,313.1 \\
213.0 \\
47.7 \\
382.3 \\
1,720.0 \\
100.7 \\
\end{array}$ & $\begin{array}{r}2,029.9 \\
202.6 \\
31.4 \\
.2 .1 \\
42.1 \\
260.1 \\
31.1 \\
\end{array}$ & $\begin{array}{r}1,305.4 \\
307.4 \\
91.9 \\
-- \\
62.3 \\
306.9 \\
2 b .6\end{array}$ & $\begin{array}{r}596.5 \\
138.8 \\
35.7 \\
2.1 \\
31.8 \\
112.0 \\
2.0 \\
\end{array}$ & $\begin{array}{r}9,926.3 \\
664.3 \\
54.0 \\
43.5 \\
246.1 \\
1,041.0 \\
39.0 \\
\end{array}$ \\
\hline Subtotal & $17,634.9$ & $2,599.3$ & $2,099.5$ & 921.9 & $12,014.2$ \\
\hline Total & $18,562.9$ & $2,780.9$ & $2,342.8$ & $1,040.2$ & $12,399.0$ \\
\hline Total land & $23,002.1$ & $3,658.5$ & $4,148.1$ & $1,644.4$ & $13,551.1$ \\
\hline Water-1/ & 156.5 & 28.0 & 38.8 & 6.6 & 83.1 \\
\hline Iotal land and water & $23,158.6$ & $3,686.5$ & $4,186.9$ & $1,651.0$ & $13,634.2$ \\
\hline
\end{tabular}

1/1982 National Resource Inventory, Soil Conservation Service, USDA. 
Table 10.--Area of land by county and major land use class, Indiana, 1986

\begin{tabular}{|c|c|c|c|c|c|c|c|c|}
\hline \multirow[b]{2}{*}{ Unit and county } & \multirow[b]{2}{*}{$\begin{array}{l}\text { Land } \\
\text { area }\end{array}$} & \multicolumn{4}{|c|}{ Forest land } & \multirow[b]{2}{*}{$\begin{array}{l}\text { Non forest } \\
\text { land } \\
\text { with trees }\end{array}$} & \multirow{2}{*}{$\begin{array}{l}\text { Nonforest } \\
\text { land as a } \\
\text { percent of } \\
\text { land area }\end{array}$} & \multirow[b]{2}{*}{$\begin{array}{l}\text { Sampling } \\
\text { error for } \\
\text { timberland }\end{array}$} \\
\hline & & $\begin{array}{l}\text { All } \\
\text { forest } \\
\text { land }\end{array}$ & $\begin{array}{l}\text { Reserved } \\
\text { timberland }\end{array}$ & Timberl and & $\begin{array}{l}\text { Timberland as } \\
\text { a percent of } \\
\text { land area }\end{array}$ & & & \\
\hline Lower Wabash Unit & --- & - - Thou & 5 and acres - & $---\cdot-$ & Percent & Thousand acres & Percent & Percent \\
\hline $\begin{array}{l}\text { Clay } \\
\text { Daviess } \\
\text { Gibson } \\
\text { Greene } \\
\text { Knox } \\
\text { Martin } \\
\text { Parke } \\
\text { Pike } \\
\text { Posey } \\
\text { Putnam } \\
\text { Sullivan } \\
\text { Vanderburgh } \\
\text { Vermillion } \\
\text { Vigo }\end{array}$ & $\begin{array}{l}230.5 \\
276.6 \\
313.6 \\
348.6 \\
332.6 \\
216.8 \\
284.2 \\
218.0 \\
261.8 \\
308.4 \\
287.8 \\
151.1 \\
166.4 \\
262.1 \\
\end{array}$ & $\begin{array}{r}44.7 \\
42.1 \\
42.4 \\
106.2 \\
31.0 \\
131.9 \\
91.0 \\
84.4 \\
49.9 \\
76.7 \\
65.5 \\
25.7 \\
35.0 \\
51.1 \\
\end{array}$ & $\begin{array}{l}0.4 \\
0.5 \\
0.4 \\
0.9 \\
0.2 \\
3.5 \\
3.4 \\
0.5 \\
4.8 \\
0.2 \\
0.8 \\
1.0 \\
0.3 \\
0.3 \\
\end{array}$ & $\begin{array}{r}44.3 \\
41.6 \\
42.0 \\
105.3 \\
30.8 \\
128.4 \\
87.6 \\
83.9 \\
45.1 \\
76.5 \\
64.7 \\
24.7 \\
34.7 \\
50.8 \\
\end{array}$ & $\begin{array}{r}19.2 \\
15.0 \\
13.4 \\
30.2 \\
9.3 \\
59.2 \\
30.8 \\
38.5 \\
17.2 \\
24.8 \\
22.5 \\
16.3 \\
20.9 \\
19.4 \\
\end{array}$ & $\begin{array}{r}15.7 \\
9.9 \\
14.3 \\
20.9 \\
17.3 \\
6.4 \\
12.8 \\
8.6 \\
9.5 \\
16.0 \\
9.9 \\
18.7 \\
6.2 \\
15.4 \\
\end{array}$ & $\begin{array}{r}6.8 \\
3.6 \\
4.6 \\
6.0 \\
5.2 \\
3.0 \\
4.5 \\
3.9 \\
3.6 \\
5.2 \\
3.4 \\
12.4 \\
3.7 \\
5.9 \\
\end{array}$ & $\begin{array}{r}11.11 \\
11.46 \\
11.41 \\
7.20 \\
13.32 \\
6.52 \\
7.90 \\
8.07 \\
11.01 \\
8.45 \\
9.19 \\
14.87 \\
12.55 \\
10.37 \\
\end{array}$ \\
\hline Total & $3,658.5$ & 877.6 & 17.2 & 860.4 & 23.5 & 181.6 & 5.0 & 2.52 \\
\hline $\begin{array}{l}\text { Knobs Unit } \\
\text { Brown } \\
\text { Clark } \\
\text { Crawford } \\
\text { Dubois } \\
\text { Floyd } \\
\text { Harrison } \\
\text { Jackson } \\
\text { Lawrence } \\
\text { Monroe } \\
\text { Morgan } \\
\text { Orange } \\
\text { Owen } \\
\text { Perry } \\
\text { Scott } \\
\text { Spencer } \\
\text { Warrick } \\
\text { Washington }\end{array}$ & $\begin{array}{r}199.2 \\
240.8 \\
196.1 \\
274.8 \\
95.8 \\
311.0 \\
327.8 \\
289.3 \\
246.4 \\
261.6 \\
254.7 \\
247.1 \\
244.2 \\
122.5 \\
256.2 \\
250.4 \\
330.2 \\
\end{array}$ & $\begin{array}{r}150.0 \\
85.9 \\
120.8 \\
93.8 \\
34.9 \\
135.2 \\
125.2 \\
126.8 \\
130.8 \\
88.2 \\
131.5 \\
110.3 \\
153.8 \\
45.6 \\
65.0 \\
84.0 \\
123.5 \\
\end{array}$ & $\begin{array}{r}18.4 \\
1.0 \\
1.5 \\
0.8 \\
0.4 \\
3.1 \\
4.6 \\
1.5 \\
13.3 \\
1.5 \\
2.4 \\
2.8 \\
1.3 \\
2.6 \\
2.5 \\
2.6 \\
3.9 \\
\end{array}$ & $\begin{array}{r}131.6 \\
84.9 \\
119.3 \\
93.0 \\
34.5 \\
132.1 \\
120.6 \\
125.3 \\
117.5 \\
86.7 \\
129.1 \\
107.5 \\
152.5 \\
43.0 \\
62.5 \\
81.4 \\
119.6 \\
\end{array}$ & $\begin{array}{l}66.1 \\
35.3 \\
60.8 \\
33.8 \\
36.0 \\
42.5 \\
36.8 \\
43.3 \\
47.7 \\
33.1 \\
50.7 \\
43.5 \\
62.4 \\
35.1 \\
24.4 \\
32.5 \\
36.2 \\
\end{array}$ & $\begin{array}{r}10.4 \\
14.2 \\
7.8 \\
15.2 \\
14.2 \\
17.1 \\
17.2 \\
15.4 \\
13.3 \\
18.3 \\
13.3 \\
15.7 \\
9.7 \\
6.9 \\
14.8 \\
16.8 \\
23.0 \\
\end{array}$ & $\begin{array}{r}5.2 \\
5.9 \\
4.0 \\
5.5 \\
14.8 \\
5.5 \\
5.2 \\
5.3 \\
5.4 \\
7.0 \\
5.2 \\
6.4 \\
4.0 \\
5.6 \\
5.8 \\
6.7 \\
7.0 \\
\end{array}$ & $\begin{array}{l}3.02 \\
3.76 \\
3.17 \\
3.59 \\
5.90 \\
3.01 \\
3.15 \\
3.09 \\
3.20 \\
3.72 \\
3.05 \\
3.34 \\
2.80 \\
5.28 \\
4.38 \\
3.84 \\
3.17 \\
\end{array}$ \\
\hline Total & $4,148.1$ & $1,805.3$ & 64.2 & $1,741,1$ & 42.0 & 243.3 & 5.9 & .83 \\
\hline $\begin{array}{l}\text { Upland Flats Unit } \\
\text { Dearborn } \\
\text { Fayette } \\
\text { Franklin } \\
\text { Jefferson } \\
\text { Jennings } \\
\text { Ohio } \\
\text { Ripley } \\
\text { Switzerland } \\
\text { Union }\end{array}$ & $\begin{array}{r}196.7 \\
137.8 \\
246.9 \\
232.0 \\
241.8 \\
55.8 \\
286.3 \\
143.1 \\
104.0 \\
\end{array}$ & $\begin{array}{l}91.1 \\
33.6 \\
82.2 \\
87.3 \\
95.7 \\
27.9 \\
88.8 \\
75.7 \\
21.9 \\
\end{array}$ & $\begin{array}{r}-- \\
0.1 \\
1.8 \\
9.0 \\
8.1 \\
-- \\
13.1 \\
0.5 \\
0.5 \\
\end{array}$ & $\begin{array}{l}91.1 \\
33.5 \\
80.4 \\
78.3 \\
87.6 \\
27.9 \\
75.7 \\
75.2 \\
21.4 \\
\end{array}$ & $\begin{array}{l}46.3 \\
24.3 \\
32.6 \\
33.8 \\
36.2 \\
50.0 \\
26.4 \\
52.6 \\
20.6 \\
\end{array}$ & $\begin{array}{r}12.6 \\
8.5 \\
17.4 \\
19.7 \\
15.0 \\
3.6 \\
25.9 \\
8.9 \\
6.7 \\
\end{array}$ & $\begin{array}{l}6.4 \\
6.2 \\
7.0 \\
8.5 \\
6.2 \\
6.5 \\
9.0 \\
6.2 \\
6.4 \\
\end{array}$ & $\begin{array}{r}8.86 \\
14.62 \\
9.43 \\
9.56 \\
9.04 \\
16.02 \\
9.72 \\
9.76 \\
18.29 \\
\end{array}$ \\
\hline Total & $1,644.4$ & 604.2 & 33.1 & 571.1 & 34.7 & 118.3 & 7.2 & 3.54 \\
\hline
\end{tabular}


(Table 10 continued)

\begin{tabular}{|c|c|c|c|c|c|c|c|c|}
\hline \multirow[b]{2}{*}{ Unit and county } & \multirow[b]{2}{*}{$\begin{array}{l}\text { Land } \\
\text { area }\end{array}$} & \multicolumn{4}{|c|}{ Forest land } & \multirow[b]{2}{*}{$\begin{array}{l}\text { Non forest } \\
\text { land } \\
\text { with trees }\end{array}$} & \multirow{2}{*}{$\begin{array}{l}\text { Nonforest } \\
\text { land as a } \\
\text { percent of } \\
\text { land area }\end{array}$} & \multirow[b]{2}{*}{$\begin{array}{l}\text { Sampling } \\
\text { error for } \\
\text { timberland }\end{array}$} \\
\hline & & $\begin{array}{l}\text { All } \\
\text { forest } \\
\text { land }\end{array}$ & $\begin{array}{l}\text { Reserved } \\
\text { timberl and }\end{array}$ & Timberl and & $\begin{array}{l}\text { Timberland as } \\
\text { a percent of } \\
\text { land area }\end{array}$ & & & \\
\hline & -- & - - Thou & and acres & $--\cdot-$ & Percent & Thousand acres & Percent & Percent \\
\hline \multicolumn{9}{|l|}{ Northern Unit } \\
\hline Adams & 217.5 & 14.2 & 0.1 & 14.1 & 6.5 & 2.1 & 1.0 & 21.78 \\
\hline Allen & 421.7 & 30.5 & 0.7 & 29.8 & 7.1 & 23.8 & 5.6 & 14.98 \\
\hline Barthol omew & 261.5 & 47.1 & 2.3 & 44.8 & 17.1 & 6.9 & 2.6 & 12.22 \\
\hline Benton & 260.2 & 1.5 & -- & 1.5 & 0.6 & 0.4 & 0.2 & 66.77 \\
\hline Blackford & 106.0 & 9.4 & - & 9.4 & 8.9 & 1.3 & 1.2 & 26.67 \\
\hline Boone & 271.0 & 15.3 & 0.1 & 15.2 & 5.6 & 4.1 & 1.5 & 20.97 \\
\hline Carroll & 238.1 & 18.5 & 0.1 & 18.4 & 7.7 & 6.1 & 2.6 & 19.06 \\
\hline Cass & 265.1 & 24.3 & 0.1 & 24.2 & 9.1 & 5.8 & 2.2 & 16.62 \\
\hline $\mathrm{Cl}$ inton & 259.3 & 9.3 & 0.1 & 9.2 & 3.5 & 2.4 & 0.9 & 26.96 \\
\hline Decatur & 238.6 & 24.3 & 0.2 & 24.1 & 10.1 & 3.4 & 1.4 & 16.66 \\
\hline De Kalb & 232.4 & 27.4 & 0.1 & 27.3 & 11.7 & 5.9 & 2.5 & 15.65 \\
\hline Del aware & 250.8 & 15.9 & 0.1 & 15.8 & 6.3 & 4.6 & 1.8 & 20.57 \\
\hline Elkhart & 298.4 & 29.4 & 0.3 & 29.1 & 9.7 & 8.4 & 2.8 & 15.16 \\
\hline Fountain & 254.6 & 37.4 & 0.8 & 36.6 & 14.4 & 8.4 & 3.3 & 13.52 \\
\hline Fulton & 236.3 & 20.5 & 0.1 & 20.4 & 8.6 & 5.1 & 2.2 & 18.11 \\
\hline Grant & 265.5 & 18.3 & 0.1 & 18.2 & 6.9 & 4.6 & 1.7 & 19.17 \\
\hline Hamilton & 255.0 & 20.4 & 0.4 & 20.0 & 7.8 & 5.3 & 2.1 & 18.29 \\
\hline Hancock & 196.5 & 11.9 & 0.1 & 11.8 & 6.0 & 4.9 & 2.5 & 23.81 \\
\hline Hendricks & 261.7 & 17.8 & 0.1 & 17.7 & 6.8 & 5.1 & 1.9 & 19.44 \\
\hline Henry & 251.8 & 20.7 & 0.1 & 20.6 & 8.2 & 8.4 & 3.3 & 18.02 \\
\hline Howard & 187.6 & 8.7 & 0.1 & 8.6 & 4.6 & 7.4 & 3.9 & 27.88 \\
\hline Huntington & 234.4 & 23.1 & 0.2 & 22.9 & 9.8 & 5.3 & 2.3 & 17.09 \\
\hline Jasper & 359.0 & 27.9 & 0.9 & 27.0 & 7.5 & 6.6 & 1.8 & 15.74 \\
\hline Jay & 245.8 & 24.1 & 0.1 & 24.0 & 9.8 & 3.0 & 1.2 & 16.69 \\
\hline Johnson & 205.8 & 20.2 & 0.1 & 20.1 & 9.8 & 5.7 & 2.8 & 18.24 \\
\hline Kosciusko & 345.2 & 33.1 & 0.1 & 33.0 & 9.6 & 5.8 & 1.7 & 14.24 \\
\hline La Grange & 243.2 & 36.0 & 0.1 & 35.9 & 14.8 & 10.6 & 4.4 & 13.65 \\
\hline Lake & 320.5 & 18.5 & 0.7 & 17.8 & 5.6 & 11.5 & 3.6 & 19.38 \\
\hline La Porte & 384.2 & 42.2 & 0.8 & 41.4 & 10.8 & 11.2 & 2.9 & 12.71 \\
\hline Madison & 289.8 & 13.1 & 0.1 & 13.0 & 4.5 & 4.4 & 1.5 & 22.68 \\
\hline Marion & 252.9 & 1.9 & 1.0 & 0.9 & 0.4 & 53.3 & 21.1 & 86.20 \\
\hline Marshall & 284.3 & 31.1 & 0.1 & 31.0 & 10.9 & 6.5 & 2.3 & 14.69 \\
\hline Miami & 240.9 & 25.5 & 0.1 & 25.4 & 10.5 & 4.1 & 1.7 & 16.23 \\
\hline Montgomery & 323.1 & 25.7 & 1.7 & 24.0 & 7.4 & 7.2 & 2.2 & 16.69 \\
\hline Newton & 256.6 & 19.5 & 1.6 & 17.9 & 7.0 & 2.1 & 0.8 & 19.33 \\
\hline Noble & 264.0 & 33.6 & 1.3 & 32.3 & 12.2 & 7.9 & 3.0 & 14.39 \\
\hline Porter & 267.7 & 37.9 & 7.3 & 30.6 & 11.4 & 13.6 & 5.1 & 14.78 \\
\hline Pulaski & 278.1 & 29.3 & 2.3 & 27.0 & 9.7 & 5.7 & 2.0 & 15.74 \\
\hline Randolph & 290.2 & 19.8 & 0.1 & 19.7 & 6.8 & 4.2 & 1.4 & 18.42 \\
\hline Rush & 261.2 & 12.6 & 0.2 & 12.4 & 4.7 & 7.4 & 2.8 & 23.22 \\
\hline St. Joseph & 293.9 & 23.3 & 0.8 & 22.5 & 7.7 & 10.3 & 3.5 & 17.24 \\
\hline Shelby & 264.0 & 12.6 & 0.1 & 12.5 & 4.7 & 2.1 & 0.8 & 23.13 \\
\hline Starke & 198.0 & 27.1 & 0.2 & 26.9 & 13.6 & 6.9 & 3.5 & 15.77 \\
\hline Steuben & 196.8 & 32.5 & 1.0 & 31.5 & 16.0 & 7.9 & 4.0 & 14.57 \\
\hline Tippecanoe & 321.1 & 22.8 & 0.2 & 22.6 & 7.0 & 7.8 & 2.4 & 17.20 \\
\hline Tipton & 166.6 & 4.8 & 0.1 & 4.7 & 2.8 & 4.2 & 2.5 & 37.72 \\
\hline Wabash & 265.6 & 24.2 & 0.2 & 24.0 & 9.0 & 8.9 & 3.4 & 16.69 \\
\hline Warren & 234.5 & 23.9 & 0.1 & 23.8 & 10.1 & 5.8 & 2.5 & 16.76 \\
\hline Wayne & 258.3 & 32.0 & 0.1 & 31.9 & 12.3 & 17.1 & 6.6 & 14.48 \\
\hline Wells & 236.9 & 17.3 & 1.1 & 16.2 & 6.8 & 3.4 & 1.4 & 20.32 \\
\hline White & 324.0 & 13.1 & 0.1 & 13.0 & 4.0 & 4.9 & 1.5 & 22.68 \\
\hline Whitley & 214.9 & 20.6 & 0.1 & 20.5 & 9.5 & 5.0 & 2.3 & 18.06 \\
\hline Total & $13,551.1$ & $1,152.1$ & 28.9 & $1,123.2$ & 8.3 & 384.8 & 2.8 & 2.44 \\
\hline All counties & $23,002.1$ & $4,439.2$ & 143.4 & $4,295.8$ & 18.7 & 928.0 & 4.0 & 1.00 \\
\hline
\end{tabular}


Table 11.--Area of timberland by county and ownership class, Indiana, 1986

(In thousand acres)

\begin{tabular}{|c|c|c|c|c|c|c|c|c|c|}
\hline \multirow[b]{2}{*}{ Unit and county } & \multirow[b]{2}{*}{$\begin{array}{c}\text { All } \\
\text { owners }\end{array}$} & \multirow[b]{2}{*}{$\begin{array}{l}\text { National } \\
\text { forest }\end{array}$} & \multicolumn{7}{|c|}{ Ownership class } \\
\hline & & & \multirow[t]{2}{*}{$\begin{array}{c}\text { Misc. } \\
\text { federal }\end{array}$} & State & $\begin{array}{l}\text { County \& } \\
\text { municipal }\end{array}$ & $\begin{array}{c}\text { Forest } \\
\text { industry }\end{array}$ & Farmer & $\begin{array}{l}\text { Misc. } \\
\text { private- } \\
\text { corp. }\end{array}$ & $\begin{array}{l}\text { Misc. } \\
\text { private- } \\
\text { indiv. }\end{array}$ \\
\hline Lower Wabash Unit & & & & & & & & & \\
\hline Clay & 44.3 & -- & 2.9 & -- & 0.6 & -- & 17.4 & 6.8 & 16.6 \\
\hline Oaviess & 41.6 & -- & 2.6 & 3.6 & 0.4 & 0.2 & 13.8 & 6.7 & 14.3 \\
\hline Gibson & 42.0 & -- & 1.9 & 0.2 & 0.5 & -- & 17.5 & 6.4 & 15.5 \\
\hline Greene & 105.3 & -- & 7.1 & 4.5 & 0.9 & -- & 37.1 & 18.4 & 37.3 \\
\hline knox & 30.8 & -- & 2.0 & 0.4 & 0.4 & -- & 13.0 & 1.9 & 13.1 \\
\hline Martin & 128.4 & 9.0 & 12.7 & 7.6 & 1.0 & 1.1 & 40.5 & 15.2 & $41 \cdot 3$ \\
\hline Parke & 87.6 & -- & 3.5 & 0.3 & 0.9 & 0.7 & 33.2 & 15.3 & 33.7 \\
\hline Pike & 83.9 & -- & 5.0 & 4.0 & 0.7 & -- & 28.5 & 15.7 & 30.0 \\
\hline Posey & 45.1 & -- & 3.1 & 1.7 & 0.5 & -- & 17.1 & 5.9 & 16.8 \\
\hline Putnam & 76.5 & -. & 6.7 & 2.1 & 1.0 & 0.1 & 29.2 & 3.7 & 33.7 \\
\hline Sullivan & 64.7 & -- & 4.3 & 4.9 & 0.5 & - & 20.6 & 11.6 & 22.8 \\
\hline Vanderburgh & 24.7 & - - & 1.3 & - & 0.3 & -- & 10.2 & 2.3 & 10.6 \\
\hline Vermillion & 34.7 & -- & 3.1 & -- & 0.2 & -- & 14.1 & 4.1 & 13.2 \\
\hline Vigo & 50.8 & -- & 3.0 & 0.2 & 0.4 & -- & 20.3 & 8.9 & 18.0 \\
\hline Total & 860.4 & 9.0 & 59.2 & 29.5 & 8.3 & 2.1 & 312.5 & 122.9 & 316.9 \\
\hline \multicolumn{10}{|l|}{ Knobs Unit } \\
\hline Brown & 131.6 & 14.4 & 10.3 & 23.3 & 0.3 & 2.1 & 29.8 & 9.3 & 42.1 \\
\hline Clark & 84.9 & -- & 4.1 & 15.9 & 0.3 & - & 23.7 & 7.7 & 33.2 \\
\hline Crawford & 119.3 & 19.5 & 8.3 & 12.6 & 0.3 & 2.4 & 27.2 & 8.2 & 40.8 \\
\hline Oubois & 93.0 & 0.3 & 6.2 & 4.5 & 0.3 & 0.1 & 30.8 & 9.8 & 41.0 \\
\hline Floyd & 34.5 & - & -. & -- & 0.1 & 0.2 & 14.5 & 4.3 & 15.4 \\
\hline Harrison & 132.1 & -- & - & 13.9 & 0.5 & 1.0 & 41.4 & 11.7 & 63.6 \\
\hline Jackson & 120.6 & 20.0 & 4.1 & 6.9 & 0.5 & 0.3 & 32.7 & 9.2 & 46.9 \\
\hline Lawrence & 125.3 & 14.9 & 2.1 & -- & 0.3 & 0.6 & 39.3 & 12.5 & 55.6 \\
\hline Monroe & 117.5 & 8.0 & 8.3 & 18.9 & 0.3 & 0.6 & 30.6 & 9.2 & 41.6 \\
\hline Morgan & 86.7 & -- & -- & 5.2 & 0.6 & 1.1 & 28.5 & 8.3 & 43.0 \\
\hline Orange & 129.1 & 26.0 & 12.4 & 1.3 & 0.4 & 0.7 & 31.5 & 8.9 & 47.9 \\
\hline Owen & 107.5 & -- & 6.2 & 5.5 & 0.2 & 0.4 & 40.8 & 11.9 & 42.5 \\
\hline Perry & 152.5 & 53.9 & -- & 3.4 & 0.3 & 2.6 & 32.6 & 10.3 & 49.4 \\
\hline$s \cot t$ & 43.0 & -- & - & 5.3 & 0.3 & - & 13.4 & 4.1 & 19.9 \\
\hline Spencer & 62.5 & -- & - & -- & 0.4 & 0.2 & 22.4 & 6.7 & 32.8 \\
\hline Warrick & 81.4 & -- & -- & -- & 0.5 & 0.5 & 30.2 & 8.9 & 41.3 \\
\hline Washington & 119.6 & -- & -- & 12.6 & 0.3 & 0.7 & 45.1 & 13.6 & 47.3 \\
\hline Total & $1,741.1$ & 157.0 & 62.0 & 129.3 & 5.9 & 13.5 & 514.5 & 154.6 & 704.3 \\
\hline \multicolumn{10}{|l|}{ Upland Fiats Unit } \\
\hline Dearborn & 91.1 & - & 2.2 & 0.3 & 0.5 & -- & 45.6 & 5.2 & 36.3 \\
\hline Fayet te & 33.5 & -- & 0.8 & 0.1 & 0.4 & -- & 16.7 & 1.3 & 14.2 \\
\hline Franklin & 80.4 & - & 1.8 & 0.2 & 0.6 & -- & 38.6 & 3.6 & 35.6 \\
\hline Jefferson & 78.3 & -- & 2.0 & 0.1 & 0.1 & -- & 36.3 & 2.8 & 37.0 \\
\hline Jennings & 87.6 & $\cdots$ & 1.6 & 6.9 & 0.6 & -- & 38.1 & 5.0 & 35.4 \\
\hline Ohio & 27.9 & -- & 0.7 & -- & 0.2 & -- & 14.3 & 1.5 & 11.2 \\
\hline Ripley & 75.7 & -- & 2.0 & -- & 0.1 & -- & 33.1 & 2.5 & 38.0 \\
\hline Switzerland & 75.2 &.- & 1.8 & -- & 0.1 & -- & 37.6 & 3.6 & 32.1 \\
\hline Union & 21.4 & -- & 0.6 & -- & 0.1 & -- & 10.0 & 1.0 & 9.7 \\
\hline Total & 571.1 & $\cdots$ & 13.5 & 7.6 & 2.7 & $\cdots$ & 271.3 & 26.5 & 249.5 \\
\hline
\end{tabular}


(Table 11 continued)

\begin{tabular}{|c|c|c|c|c|c|c|c|c|c|}
\hline \multirow[b]{2}{*}{ Unit and county } & \multirow[b]{2}{*}{$\begin{array}{c}\text { All } \\
\text { owners }\end{array}$} & \multicolumn{7}{|c|}{ Ownership class } & \multirow[b]{2}{*}{$\begin{array}{l}\text { Misc. } \\
\text { private- } \\
\text { indiv. }\end{array}$} \\
\hline & & $\begin{array}{c}\text { National } \\
\text { forest }\end{array}$ & $\begin{array}{c}\text { Misc. } \\
\text { federal }\end{array}$ & State & $\begin{array}{l}\text { County \& } \\
\text { municipal }\end{array}$ & $\begin{array}{c}\text { Forest } \\
\text { industry }\end{array}$ & Farmer & $\begin{array}{l}\text { Misc. } \\
\text { private- } \\
\text { corp. }\end{array}$ & \\
\hline \multicolumn{10}{|l|}{ Northern Unit } \\
\hline Adams & 14.1 & -- & 0.3 & -- & 0.1 & 0.1 & 7.9 & 1.3 & 4.4 \\
\hline Allen & 29.8 & -- & 0.4 & -- & 0.3 & 0.2 & 15.0 & 3.1 & 10.8 \\
\hline Bar tholomew & 44.8 & -- & 1.0 & 0.1 & 0.4 & 0.1 & 24.8 & 4.5 & 13.9 \\
\hline Benton & 1.5 & -- & 0.0 & -- & -- & -- & 0.8 & 0.2 & 0.5 \\
\hline Blackford & 9.4 & -- & 0.2 & -- & 0.1 & -- & 5.3 & 0.9 & 2.9 \\
\hline Boone & 15.2 & -. & 0.4 & -- & 0.1 & -- & 8.1 & 1.6 & 5.0 \\
\hline Carroll & 18.4 & -- & 0.3 & 0.1 & 0.2 & -. & 10.0 & 1.7 & 6.1 \\
\hline Cass & 24.2 & -- & 0.5 & - & 0.3 & 0.2 & 13.6 & 2.0 & 7.6 \\
\hline $\mathrm{Cl}$ inton & 9.2 & -- & 0.2 & -- & 0.1 & -- & 5.0 & 0.9 & 3.0 \\
\hline Decatur & 24.1 & -- & 0.5 & -- & 0.2 & -- & 13.5 & 2.3 & 7.6 \\
\hline Oe Kalb & 27.3 & -- & 0.9 & -- & 0.3 & 0.3 & 15.3 & 2.2 & 8.3 \\
\hline Oelaware & 15.8 & -- & 0.4 & -- & 0.2 & -- & 8.3 & 1.6 & 5.3 \\
\hline Elkhart & 29.1 & -- & 0.6 & -- & 0.3 & $\ldots$ & 16.4 & 2.4 & 9.4 \\
\hline Fountain & 36.6 & -- & 1.0 & -- & 0.4 & -- & 21.2 & 2.9 & 11.1 \\
\hline Fulton & 20.4 & -. & 0.5 & 0.1 & 0.2 & 0.3 & 11.1 & 1.8 & 6.4 \\
\hline Grant & 18.2 & -- & 0.4 & -- & 0.2 & -. & 10.3 & 1.6 & 5.7 \\
\hline Hamilton & 20.0 & -- & 0.5 & -- & 0.3 & -- & 11.0 & 1.7 & 6.5 \\
\hline Hancock & 11.8 & -- & 0.3 & -- & 0.2 & -- & 6.6 & 0.9 & 3.8 \\
\hline Hendricks & 17.7 & -- & 0.4 & -- & 0.2 & -- & 9.5 & 1.8 & 5.8 \\
\hline Henry & 20.6 & - & 0.6 & 0.3 & 0.3 & 0.1 & 10.6 & 1.9 & 6.8 \\
\hline Howard & 8.6 & -- & 0.1 & -- & 0.1 & -- & 4.0 & 1.1 & 3.3 \\
\hline Huntington & 22.9 & -- & 0.5 & 0.1 & 0.2 & -- & 13.0 & 2.0 & 7.1 \\
\hline Jasper & 27.0 & -- & 0.6 & 1.4 & 0.3 & -- & 13.5 & 2.5 & 8.7 \\
\hline Jay & 24.0 & -- & 0.5 & - & 0.1 & - & 13.8 & 2.3 & 7.3 \\
\hline Johnson & 20.1 & -- & 0.4 & 0.5 & 0.2 & -- & 10.4 & 2.0 & 6.6 \\
\hline Kosciusko & 33.0 & -- & 0.6 & 0.6 & 0.2 & 0.4 & 17.9 & 3.2 & 10.1 \\
\hline La Grange & 35.9 & -- & 1.9 & 0.7 & 0.5 & - & 18.1 & 3.5 & 11.2 \\
\hline Lake & 17.8 & -- & 0.5 & 0.1 & 0.2 & - & 9.8 & 1.6 & 5.6 \\
\hline La Porte & 41.4 & -- & 1.1 & 1.0 & 0.4 & 0.1 & 21.9 & 3.8 & 13.1 \\
\hline Madison & 13.0 & -- & 0.2 & $\ldots$ & 0.1 & - & 7.4 & 1.2 & 4.1 \\
\hline Marion & 0.9 & -- & 0.0 & -- & - & -- & 0.3 & 0.1 & 0.5 \\
\hline Marshall & 31.0 & -- & 0.9 & 0.1 & 0.2 & 0.2 & 17.2 & 2.8 & 9.6 \\
\hline Miami & 25.4 & -- & 0.5 & 0.1 & 0.2 & 0.2 & 14.2 & 2.4 & 7.8 \\
\hline Montgomery & 24.0 & -. & 0.5 & -. & 0.3 & -- & 13.0 & 2.1 & 8.1 \\
\hline Newton & 17.9 & -- & 0.4 & 2.0 & 0.2 & -- & 8.3 & 1.6 & 5.4 \\
\hline Noble & 32.3 & -- & 0.9 & 0.3 & 0.3 & 0.1 & 17.2 & 3.0 & 10.5 \\
\hline Porter & 30.6 & -- & 1.0 & 0.1 & 0.5 & - & 16.1 & 2.8 & 10.1 \\
\hline Pulaski & 27.0 & -- & 0.6 & 1.2 & 0.3 & 0.1 & 13.9 & 2.3 & 8.6 \\
\hline Randolph & 19.7 & -- & 0.4 & $\ldots$ & 0.1 & 0.2 & 10.8 & 1.8 & 6.4 \\
\hline Rush & 12.4 & -- & 0.2 & -- & 0.2 & - & 6.1 & 1.3 & 4.6 \\
\hline St. Joseph & 22.5 & -- & 0.6 & -- & 0.3 & -- & 12.5 & 1.9 & 7.2 \\
\hline Shelby & 12.5 & -. & 0.3 & -- & 0.1 & -- & 7.0 & 1.2 & 3.9 \\
\hline Starke & 26.9 & -- & 0.5 & 1.0 & 0.3 & -- & 14.2 & 2.2 & 8.7 \\
\hline Steuben & 31.5 & -- & 0.9 & 0.9 & 0.4 & 0.2 & 15.8 & 3.0 & 10.3 \\
\hline Tippecanoe & 22.6 & -- & 0.5 & -- & 0.3 & - & 12.3 & 1.9 & 7.6 \\
\hline Tipton & 4.7 & -- & 0.1 & -- & 0.1 & -- & 2.1 & 0.5 & 1.9 \\
\hline Wabash & 24.0 & -. & 0.8 & 0.3 & 0.3 &.- & 12.3 & 2.3 & 8.0 \\
\hline Warren & 23.8 & -- & 0.5 & -- & 0.2 & -- & 13.2 & 2.2 & 7.7 \\
\hline Wayne & 31.9 & -- & 1.0 & -- & 0.4 & -- & 17.7 & 2.8 & 10.0 \\
\hline Wells & 16.2 & -. & 0.4 & -- & 0.2 & -- & 8.9 & 1.5 & 5.2 \\
\hline White & 13.0 & -- & 0.5 & -- & 0.2 & -- & 6.8 & 1.2 & 4.3 \\
\hline Whitley & 20.5 & -- & 0.6 & -- & 0.2 & -- & 11.6 & 1.7 & 6.4 \\
\hline Total & $1,123.2$ & -- & 27.9 & 11.0 & 12.0 & 2.8 & 605.6 & 103.1 & 360.8 \\
\hline All counties & $4,295.8$ & 166.0 & 162.6 & 177.4 & 28.9 & 18.4 & $1,703.9$ & 407.1 & $1,631.5$ \\
\hline
\end{tabular}




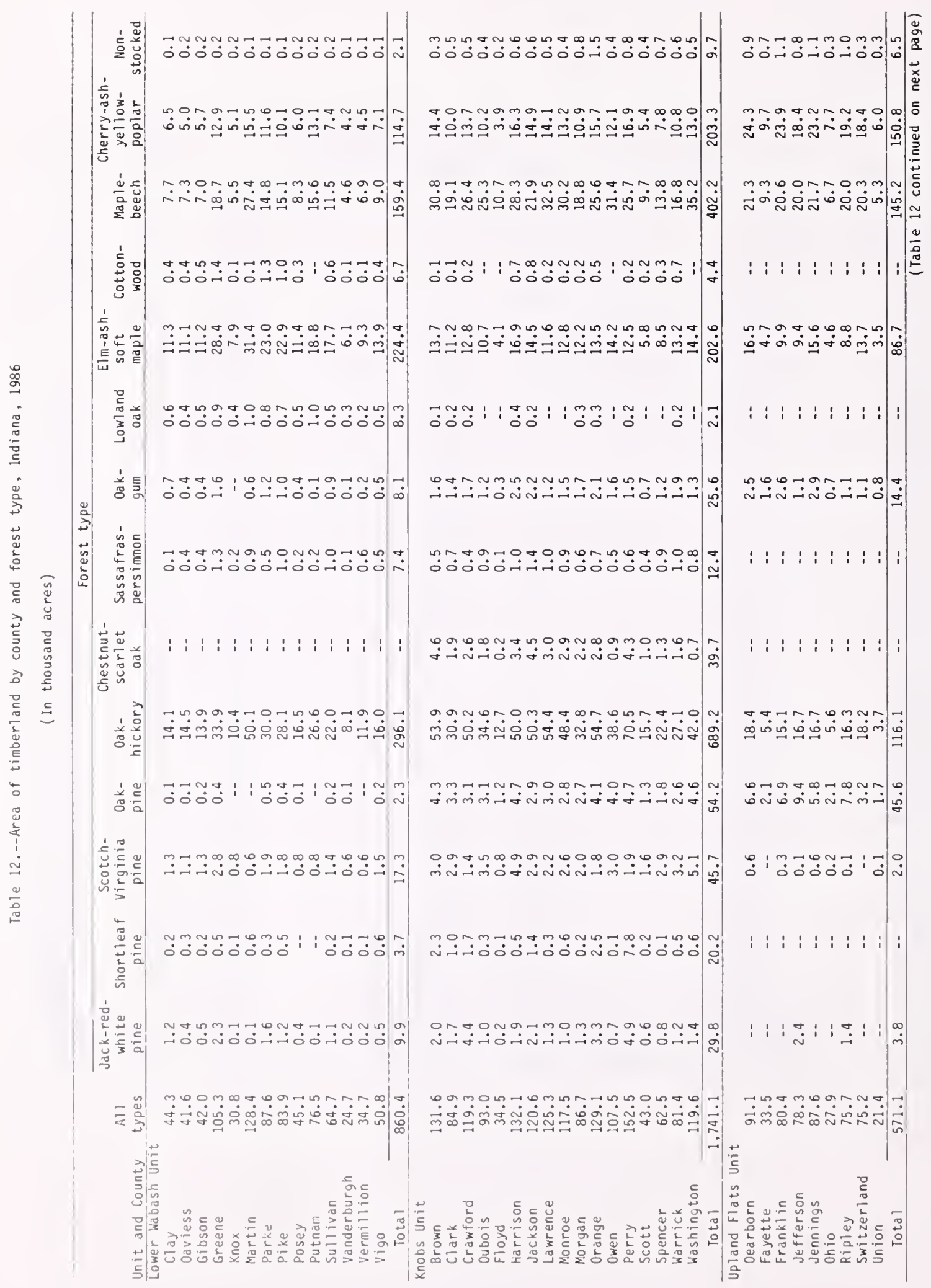




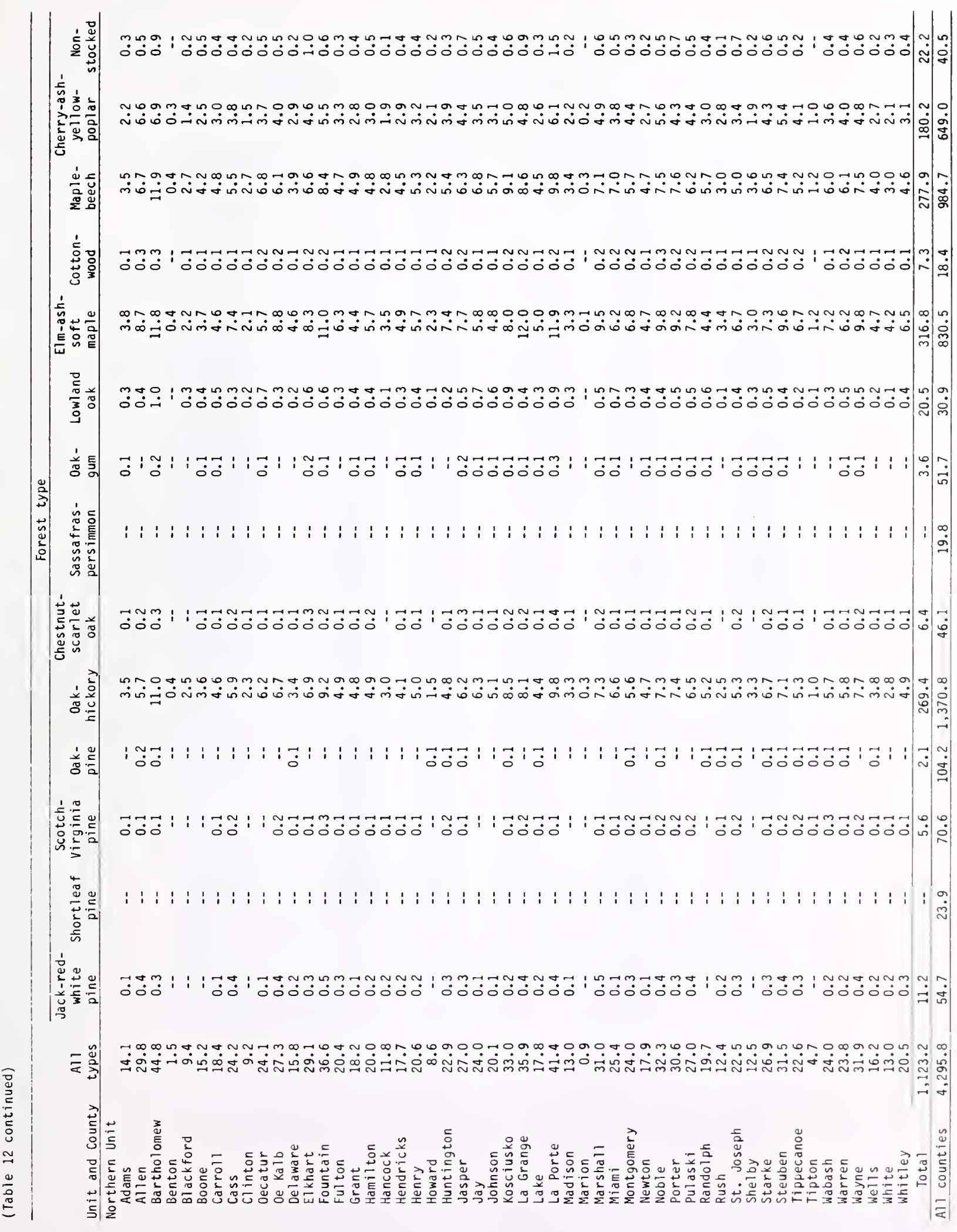


Table 13.--Area of timberland by county and stand-size class, Indian, 1986

(In thousand acres)

\begin{tabular}{|c|c|c|c|c|c|}
\hline \multirow[b]{2}{*}{ Unit and county } & \multirow[b]{2}{*}{$\begin{array}{c}\text { All } \\
\text { stands }\end{array}$} & \multicolumn{3}{|c|}{ Stand-size class } & \multirow[b]{2}{*}{ Nonstocked } \\
\hline & & Sawtimber & Poletimber & $\begin{array}{l}\text { Sapling \& } \\
\text { seedling }\end{array}$ & \\
\hline $\begin{array}{l}\text { Lower Wabash Unit } \\
\text { Clay } \\
\text { Daviess } \\
\text { Gibson } \\
\text { Greene } \\
\text { Knox } \\
\text { Martin } \\
\text { Parke } \\
\text { Pike } \\
\text { Posey } \\
\text { Putnam } \\
\text { Sullivan } \\
\text { Vanderburgh } \\
\text { Vermillion } \\
\text { Vigo }\end{array}$ & $\begin{array}{r}44.3 \\
41.6 \\
42.0 \\
105.3 \\
30.8 \\
128.4 \\
87.6 \\
83.9 \\
45.1 \\
76.5 \\
64.7 \\
24.7 \\
34.7 \\
50.8 \\
\end{array}$ & $\begin{array}{l}30.1 \\
27.2 \\
26.1 \\
65.5 \\
19.2 \\
98.3 \\
61.0 \\
54.8 \\
33.1 \\
56.6 \\
42.0 \\
16.2 \\
21.7 \\
30.7\end{array}$ & $\begin{array}{r}6.0 \\
5.8 \\
6.2 \\
17.0 \\
3.3 \\
12.2 \\
12.0 \\
13.2 \\
4.5 \\
6.2 \\
9.2 \\
3.0 \\
4.6 \\
8.8 \\
\end{array}$ & $\begin{array}{r}8.1 \\
8.4 \\
9.5 \\
22.6 \\
8.1 \\
17.8 \\
14.5 \\
15.8 \\
7.3 \\
13.5 \\
13.3 \\
5.4 \\
8.3 \\
11.2 \\
\end{array}$ & $\begin{array}{l}0.1 \\
0.2 \\
0.2 \\
0.2 \\
0.2 \\
0.1 \\
0.1 \\
0.1 \\
0.2 \\
0.2 \\
0.2 \\
0.1 \\
0.1 \\
0.1 \\
\end{array}$ \\
\hline Total & 860.4 & 582.5 & 112.0 & 163.8 & 2.1 \\
\hline $\begin{array}{l}\text { Knobs Unit } \\
\text { Brown } \\
\text { Clark } \\
\text { Crawford } \\
\text { Dubois } \\
\text { Floyd } \\
\text { Harrison } \\
\text { Jackson } \\
\text { Lawrence } \\
\text { Monroe } \\
\text { Morgan } \\
\text { Orange } \\
\text { Owen } \\
\text { Perry } \\
\text { Scott } \\
\text { Spencer } \\
\text { Warrick } \\
\text { Washington }\end{array}$ & $\begin{array}{r}131.6 \\
84.9 \\
119.3 \\
93.0 \\
34.5 \\
132.1 \\
120.6 \\
125.3 \\
117.5 \\
86.7 \\
129.1 \\
107.5 \\
152.5 \\
43.0 \\
62.5 \\
81.4 \\
119.6 \\
\end{array}$ & $\begin{array}{r}93.6 \\
56.3 \\
76.2 \\
61.6 \\
24.7 \\
84.7 \\
75.3 \\
89.4 \\
79.6 \\
54.4 \\
86.3 \\
71.9 \\
101.7 \\
27.2 \\
38.5 \\
44.9 \\
82.4 \\
\end{array}$ & $\begin{array}{r}18.6 \\
11.9 \\
17.6 \\
11.8 \\
3.8 \\
22.3 \\
21.4 \\
15.7 \\
18.4 \\
12.3 \\
19.2 \\
13.4 \\
22.9 \\
6.4 \\
8.9 \\
14.5 \\
14.9 \\
\end{array}$ & $\begin{array}{r}19.0 \\
16.3 \\
24.9 \\
19.1 \\
5.9 \\
24.4 \\
23.2 \\
19.7 \\
19.2 \\
19.3 \\
22.0 \\
21.8 \\
27.0 \\
9.1 \\
14.6 \\
21.3 \\
21.9 \\
\end{array}$ & $\begin{array}{l}0.4 \\
0.4 \\
0.6 \\
0.5 \\
0.1 \\
0.7 \\
0.7 \\
0.5 \\
0.3 \\
0.7 \\
1.6 \\
0.4 \\
0.9 \\
0.3 \\
0.5 \\
0.7 \\
0.4 \\
\end{array}$ \\
\hline Total & $1,741.1$ & $1,148.7$ & 254.0 & 328.7 & 9.7 \\
\hline $\begin{array}{l}\text { Upland Flats Unit } \\
\text { Dearborn } \\
\text { Fayette } \\
\text { Franklin } \\
\text { Jefferson } \\
\text { Jennings } \\
\text { Ohio } \\
\text { Ripley } \\
\text { Switzerland } \\
\text { Union }\end{array}$ & $\begin{array}{l}91.1 \\
33.5 \\
80.4 \\
78.3 \\
87.6 \\
27.9 \\
75.7 \\
75.2 \\
21.4\end{array}$ & $\begin{array}{r}43.9 \\
12.8 \\
31.7 \\
45.9 \\
42.2 \\
12.8 \\
41.0 \\
47.2 \\
7.9 \\
\end{array}$ & $\begin{array}{r}20.7 \\
8.2 \\
18.6 \\
12.9 \\
20.0 \\
6.2 \\
11.9 \\
10.4 \\
5.0 \\
\end{array}$ & $\begin{array}{r}25.6 \\
11.9 \\
29.0 \\
18.7 \\
24.3 \\
8.6 \\
21.8 \\
17.3 \\
8.1 \\
\end{array}$ & $\begin{array}{l}0.9 \\
0.6 \\
1.1 \\
0.8 \\
1.1 \\
0.3 \\
1.0 \\
0.3 \\
0.4 \\
\end{array}$ \\
\hline Total & 571.1 & 285.4 & 113.9 & 165.3 & 6.5 \\
\hline
\end{tabular}

(Table 13 continued on next page) 
(Table 13 continued)

\begin{tabular}{|c|c|c|c|c|c|}
\hline \multirow[b]{2}{*}{ Unit and county } & \multirow[b]{2}{*}{$\begin{array}{c}\text { All } \\
\text { stands }\end{array}$} & \multicolumn{4}{|c|}{ Stand-size class } \\
\hline & & Sawt imber & Poletimber & $\begin{array}{l}\text { Sapling \& } \\
\text { seedling }\end{array}$ & Nonstocked \\
\hline \multicolumn{6}{|l|}{ Northern Unit } \\
\hline Adams & 14.1 & 9.8 & 2.3 & 1.6 & 0.4 \\
\hline Allen & 29.8 & 17.6 & 6.0 & 5.7 & 0.5 \\
\hline 8ar tholomew & 44.8 & 32.3 & 6.5 & 5.1 & 0.9 \\
\hline 8enton & 1.5 & 1.1 & 0.3 & 0.1 & -- \\
\hline 81 ackford & 9.4 & 7.2 & 1.2 & 0.8 & 0.2 \\
\hline 8oone & 15.2 & 10.6 & 2.1 & 2.1 & 0.4 \\
\hline Carroll & 18.4 & 12.7 & 3.0 & 2.3 & 0.4 \\
\hline Cass & 24.2 & 15.8 & 4.8 & 3.2 & 0.4 \\
\hline Clinton & 9.2 & 6.8 & 1.2 & 1.0 & 0.2 \\
\hline Decatur & 24.1 & 18.3 & 3.0 & 2.3 & 0.5 \\
\hline De Kalb & 27.3 & 17.6 & 5.3 & 3.9 & 0.5 \\
\hline Delaware & 15.8 & 10.3 & 2.9 & 2.4 & 0.2 \\
\hline El khart & 29.1 & 18.1 & $\begin{array}{l}2.9 \\
5.4\end{array}$ & $\begin{array}{l}2.4 \\
4.6\end{array}$ & 1.0 \\
\hline Founta in & 36.6 & 24.9 & 6.8 & 4.3 & 0.6 \\
\hline Fulton & 20.4 & 13.7 & 3.7 & 2.7 & 0.3 \\
\hline Grant & 18.2 & 13.6 & 2.7 & 1.6 & 0.3 \\
\hline Hamil ton & 20.0 & 12.9 & 3.7 & 2.9 & 0.5 \\
\hline Hancock & 11.8 & 7.8 & 2.3 & 1.5 & 0.2 \\
\hline Hendricks & 17.7 & 11.7 & 2.9 & 2.7 & 0.4 \\
\hline Henry & 20.6 & 13.6 & 3.6 & 3.0 & 0.4 \\
\hline Howard & 8.6 & 5.3 & 1.5 & 1.7 & 0.1 \\
\hline Huntington & 22.9 & 14.3 & 4.3 & 4.0 & 0.3 \\
\hline Jasper & 27.0 & 17.3 & 4.9 & 4.1 & 0.7 \\
\hline Jay & 24.0 & 18.4 & 3.0 & 2.1 & 0.5 \\
\hline Johnson & 20.1 & 14.8 & 2.7 & 2.2 & 0.4 \\
\hline Kosciusko & 33.0 & 25.1 & 4.3 & 2.9 & 0.7 \\
\hline La Grange & 35.9 & 21.5 & 6.2 & 7.3 & 0.9 \\
\hline Lake & 17.8 & 12.2 & 3.1 & 2.2 & 0.3 \\
\hline La Porte & 41.4 & 26.4 & 7.1 & 6.5 & 1.4 \\
\hline Madison & 13.0 & 9.5 & 2.0 & 1.3 & 0.2 \\
\hline Marion & 0.9 & 0.3 & 0.3 & 0.3 & 1.2 \\
\hline Marshall & 31.0 & 20.3 & 5.6 & 4.5 & 0.6 \\
\hline Miami & 25.4 & 19.4 & 3.4 & 2.1 & 0.5 \\
\hline Montgomery & 24.0 & 15.9 & 4.7 & $\begin{array}{l}2.1 \\
3.2\end{array}$ & 0.3 \\
\hline Newton & $\begin{array}{l}24.0 \\
17.9\end{array}$ & $\begin{array}{l}13.9 \\
13.4\end{array}$ & $\begin{array}{l}4.1 \\
2.9\end{array}$ & $\begin{array}{l}3.2 \\
1.4\end{array}$ & $\begin{array}{l}0.2 \\
0.2\end{array}$ \\
\hline Noble & 32.3 & 20.7 & 6.1 & 5.0 & 0.5 \\
\hline Porter & 30.6 & 19.1 & 5.7 & 5.1 & 0.7 \\
\hline Pulaski & 27.0 & 17.9 & 5.0 & 3.6 & $\begin{array}{l}0.7 \\
0.5\end{array}$ \\
\hline Randolph & 19.7 & 15.0 & 2.5 & 1.8 & 0.4 \\
\hline Rush & 12.4 & 7.4 & 2.5 & $\begin{array}{l}1.0 \\
2.3\end{array}$ & $\begin{array}{l}0.4 \\
0.2\end{array}$ \\
\hline St. Joseph & 22.5 & 13.8 & 4.3 & 3.7 & 0.7 \\
\hline Shelby & 12.5 & 9.5 & 1.7 & 1.0 & 0.3 \\
\hline Starke & 26.9 & 18.2 & 4.7 & 3.4 & 0.6 \\
\hline Steuben & 31.5 & 20.1 & 5.8 & 5.1 & 0.5 \\
\hline Tippecanoe & 22.6 & 14.6 & 4.6 & 3.2 & 0.2 \\
\hline Tipton & 4.7 & 2.8 & 1.0 & 0.9 & -- \\
\hline Wabash & 24.0 & 15.0 & 4.6 & 4.0 & 0.4 \\
\hline Warren & 23.8 & 16.6 & 3.9 & 2.9 & 0.4 \\
\hline Wayne & 31.9 & 20.6 & 5.9 & 4.8 & 0.6 \\
\hline Wells & 16.2 & 10.9 & 3.0 & 2.1 & 0.2 \\
\hline White & 13.0 & 7.7 & 2.6 & 2.4 & 0.3 \\
\hline Whitley & 20.5 & 13.0 & 4.0 & 3.1 & 0.4 \\
\hline Total & $1,123.2$ & 753.4 & 193.6 & 154.0 & 22.2 \\
\hline All counties & $4,295.8$ & $2,770.0$ & 673.5 & 811.8 & 40.5 \\
\hline
\end{tabular}


Table 14.--Area of timberland by county and site class, Indiana, 1986

(In thousand acres)

\begin{tabular}{|c|c|c|c|c|c|c|}
\hline \multirow[b]{2}{*}{ Unit and county } & \multirow{2}{*}{$\begin{array}{c}\text { All } \\
\text { classes }\end{array}$} & \multicolumn{2}{|c|}{ Site class (cubic } & \multirow{2}{*}{$\frac{\text { feet of growt }}{85-119}$} & \multirow{2}{*}{$\frac{\text { per acre }}{50-84}$} & \multirow{2}{*}{$\frac{r \text { year }}{20-49}$} \\
\hline & & $165+$ & $120-164$ & & & \\
\hline \multicolumn{7}{|l|}{ Lower Wabash Unit } \\
\hline Clay & 44.3 & -- & 8.6 & 13.4 & 15.6 & 6.7 \\
\hline Daviess & 41.6 & -- & 6.2 & 14.1 & 15.6 & 5.7 \\
\hline Gibson & 42.0 & -- & 6.8 & 13.7 & 15.2 & 6.3 \\
\hline Greene & 105.3 & - & 18.1 & 36.2 & 36.1 & 14.9 \\
\hline Knox & 30.8 & -- & 5.1 & 8.7 & 11.9 & 5.1 \\
\hline Martin & 128.4 & -- & 17.0 & 44.8 & 53.0 & 13.6 \\
\hline Parke & 87.6 & -- & 15.7 & 29.7 & 31.2 & 11.0 \\
\hline Pike & 83.9 & -- & 13.6 & 30.2 & 29.6 & 10.5 \\
\hline Posey & 45.1 & -- & 7.2 & 14.7 & 17.4 & 5.8 \\
\hline Putnam & 76.5 & -- & 14.1 & 22.3 & 29.4 & 10.7 \\
\hline Sullivan & 64.7 & -- & 10.0 & 23.0 & 23.1 & 8.6 \\
\hline Vanderburgh & 24.7 & -- & 4.8 & 7.3 & 9.0 & 3.6 \\
\hline Vermillion & 34.7 & -- & 5.4 & 11.8 & 12.7 & 4.8 \\
\hline Vigo & 50.8 & -- & 8.4 & 17.3 & 17.8 & 7.3 \\
\hline Total & 860.4 & -- & 141.0 & 287.2 & 317.6 & 114.6 \\
\hline \multicolumn{7}{|l|}{ Knobs Unit } \\
\hline Brown & 131.6 & -- & 21.7 & 43.3 & 46.2 & 20.4 \\
\hline Clark & 84.9 & -- & 15.4 & 26.7 & 29.4 & 13.4 \\
\hline Crawford & 119.3 & -- & 20.7 & 36.7 & 39.4 & 22.5 \\
\hline Dubois & 93.0 & -- & 16.2 & 32.3 & 31.1 & 13.4 \\
\hline Floyd & 34.5 & -- & 6.4 & 14.0 & 10.5 & 3.6 \\
\hline Harrison & 132.1 & -- & 21.3 & 41.8 & 45.7 & 23.3 \\
\hline Jackson & 120.6 & -- & 17.2 & 36.2 & 44.5 & 22.7 \\
\hline Lawrence & 125.3 & -- & 20.6 & 41.7 & 43.1 & 19.9 \\
\hline Monroe & 117.5 & -- & 18.8 & 40.1 & 39.7 & 18.9 \\
\hline Morgan & 86.7 & -- & 13.5 & 26.2 & 30.9 & 16.1 \\
\hline Or ange & 129.1 & -- & 20.2 & 39.8 & 44.8 & 24.3 \\
\hline Owen & 107.5 & -- & 18.2 & 40.8 & 35.9 & 12.6 \\
\hline Perry & 152.5 & -- & 24.0 & 47.5 & 52.4 & 28.6 \\
\hline$S \cot t$ & 43.0 & - & 7.2 & 13.3 & 15.0 & 7.5 \\
\hline Spencer & 62.5 & -- & 9.8 & 18.5 & 23.3 & 10.9 \\
\hline Warrick & 81.4 & -- & 13.1 & 24.8 & 28.8 & 14.7 \\
\hline Washington & 119.6 & -- & 22.3 & 48.2 & 37.1 & 12.0 \\
\hline Iotal & $1,741.1$ & -- & 286.6 & 571.9 & 597.8 & 284.8 \\
\hline \multicolumn{7}{|l|}{ Upland Flats Unit } \\
\hline Dearborn & 91.1 & -- & 15.0 & 31.1 & 23.6 & 21.4 \\
\hline Fayet te & 33.5 & -- & 5.6 & 11.9 & 8.3 & 7.7 \\
\hline Franklin & 80.4 & -- & 12.0 & 28.8 & 21.0 & 18.6 \\
\hline Jefferson & 78.3 & -- & 18.2 & 29.6 & 18.7 & 11.8 \\
\hline Jennings & 87.6 & -- & 15.1 & 31.0 & 21.3 & 20.2 \\
\hline Onio & 27.9 & -- & 4.5 & 9.6 & 7.3 & 6.5 \\
\hline Ripley & 75.7 & -- & 16.5 & 27.8 & 19.6 & 11.8 \\
\hline Switzerland & 75.2 & -- & 16.4 & 27.3 & 18.5 & 13.0 \\
\hline Union & 21.4 & $=$ & 3.2 & 7.0 & 5.9 & 5.3 \\
\hline Total & 571.1 & - & 106.5 & 204.1 & 144.2 & 116.3 \\
\hline
\end{tabular}


(Table 14 continued)

\begin{tabular}{|c|c|c|c|c|c|c|}
\hline \multirow[b]{2}{*}{ Unit and county } & \multirow{2}{*}{$\begin{array}{c}\text { All } \\
\text { classes }\end{array}$} & \multicolumn{5}{|c|}{ Site class (cubic feet of growth per acre per year) } \\
\hline & & $165+$ & $120-164$ & $85-119$ & $50-84$ & $20-49$ \\
\hline \multicolumn{7}{|l|}{ Northern Unit } \\
\hline Adams & 14.1 & -- & 2.1 & 5.4 & 4.3 & 2.3 \\
\hline Allen & 29.8 & -- & 3.9 & 12.5 & 8.2 & 5.2 \\
\hline Barthol omew & 44.8 & -- & 6.6 & 17.2 & 13.9 & 7.1 \\
\hline Benton & 1.5 & - & 0.3 & 0.5 & 0.5 & 0.2 \\
\hline Blackford & 9.4 & - & 1.5 & 3.4 & 3.1 & 1.4 \\
\hline Boone & 15.2 & -- & 2.3 & 5.6 & 4.8 & 2.5 \\
\hline Carroll & 18.4 & -- & 2.8 & 6.9 & 5.7 & 3.0 \\
\hline Cass & 24.2 & $=$ & 3.3 & 9.9 & 7.0 & 4.0 \\
\hline Clinton & 9.2 & - & 1.4 & 3.4 & 3.0 & 1.4 \\
\hline Decatur & 24.1 & -- & 3.9 & 8.9 & 7.6 & 3.7 \\
\hline De Kalb & 27.3 & -- & 3.8 & 10.6 & 8.1 & 4.8 \\
\hline Delaware & 15.8 & -- & 2.3 & 6.2 & 4.6 & 2.7 \\
\hline Elkhart & 29.1 & -- & 3.8 & 11.3 & 8.7 & 5.3 \\
\hline Fountain & 36.6 & -- & 5.3 & 14.5 & 10.8 & 6.0 \\
\hline Fulton & 20.4 & $=-$ & 2.9 & 8.1 & 6.0 & 3.4 \\
\hline Grant & 18.2 & -- & 3.1 & 6.8 & 5.6 & 2.7 \\
\hline Hamilton & 20.0 & -. & 2.9 & 7.6 & 6.0 & 3.5 \\
\hline Hancock & 11.8 & -- & 1.8 & 4.6 & 3.5 & 1.9 \\
\hline Hendricks & 17.7 & -- & 2.4 & 6.9 & 5.4 & 3.0 \\
\hline Henry & 20.6 & -- & 2.9 & 7.8 & 6.5 & 3.4 \\
\hline Howard & 8.6 & -- & 1.3 & 3.4 & 2.4 & 1.5 \\
\hline Hunt ington & 22.9 & -- & 3.1 & 9.3 & 6.7 & 3.8 \\
\hline Jasper & 27.0 & - & 3.6 & 10.7 & 8.0 & 4.7 \\
\hline Jay & 24.0 & -- & 3.7 & 8.9 & 7.7 & 3.7 \\
\hline Johnson & 20.1 & -- & 3.0 & 7.5 & 6.5 & 3.1 \\
\hline Kosciusko & 33.0 & -- & 5.2 & 12.4 & 10.4 & 5.0 \\
\hline La Grange & 35.9 & $-\rightarrow$ & 4.2 & 12.9 & 11.8 & 7.0 \\
\hline Lake & 17.8 & -- & 2.7 & 6.8 & 5.4 & 2.9 \\
\hline La Porte & 41.4 & -- & 5.5 & 15.6 & 12.8 & 7.5 \\
\hline Madison & 13.0 & $=$ & 2.0 & 5.1 & 3.9 & 2.0 \\
\hline Marion & 0.9 & -- & 0.1 & 0.3 & 0.4 & 0.1 \\
\hline Marshall & 31.0 & $\ldots$ & 4.0 & 12.4 & 9.3 & 5.3 \\
\hline Miami & 25.4 & -- & 4.2 & 9.5 & 7.9 & 3.8 \\
\hline Mont gomery & 24.0 & -- & 3.8 & 9.5 & 6.8 & 3.9 \\
\hline Newt on & 17.9 & $\rightarrow$ & 3.1 & 6.8 & 5.3 & 2.7 \\
\hline Noble & 32.3 & -- & 4.5 & 12.8 & 9.4 & 5.6 \\
\hline Porter & 30.6 & -- & 4.2 & 11.4 & 9.7 & 5.3 \\
\hline Pulaski & 27.0 & $\ldots$ & 3.9 & 10.7 & 7.8 & 4.6 \\
\hline Randolph & 19.7 & -- & 3.1 & 7.2 & 6.4 & 3.0 \\
\hline Rush & 12.4 & -- & 1.7 & 5.1 & 3.5 & 2.1 \\
\hline St. Joseph & 22.5 & -- & 3.0 & 8.7 & 6.7 & 4.1 \\
\hline Shelby & 12.5 & $\ldots$ & 2.1 & 4.6 & 3.9 & $\begin{array}{l}4.1 \\
1.9\end{array}$ \\
\hline Starke & 26.9 & -- & 3.9 & 10.5 & 8.1 & 4.4 \\
\hline Steuben & 31.5 & -- & 4.4 & 12.1 & 9.4 & 5.6 \\
\hline Tippecanoe & 22.6 & -- & 3.4 & 9.1 & 6.4 & 3.7 \\
\hline Tipt on & 4.7 & -- & 0.7 & 1.8 & 1.4 & 0.8 \\
\hline Wabash & 24.0 & -- & 3.3 & 9.0 & 7.6 & 4.1 \\
\hline Warren & 23.8 & -- & 3.6 & 9.1 & 7.2 & 3.9 \\
\hline Wayne & 31.9 & -- & 4.1 & 12.6 & 9.8 & 5.4 \\
\hline Wells & 16.2 & -- & 2.6 & 6.3 & 4.6 & 2.7 \\
\hline White & 13.0 & -- & 1.7 & 4.9 & 4.0 & 2.4 \\
\hline Whitley & 20.5 & - & 2.6 & 8.2 & 6.2 & 3.5 \\
\hline Total & $1,123.2$ & -. & 161.6 & 433.3 & 340.7 & 187.6 \\
\hline Al1 counties & $4,295.8$ & -- & 695.7 & $1,496.5$ & $1,400.3$ & 703.3 \\
\hline
\end{tabular}


Table 15.--Area of timberland by county and stocking class of growing-stock trees, Indiana, 1986

(In thousand acres)

\begin{tabular}{|c|c|c|c|c|c|c|}
\hline \multirow[b]{2}{*}{ Unit and county } & \multirow[b]{2}{*}{$\begin{array}{c}\text { All } \\
\text { classes }\end{array}$} & \multicolumn{5}{|c|}{ Stocking percent of growing-stock trees } \\
\hline & & $\begin{array}{c}\text { Less than } \\
16.7\end{array}$ & $\begin{array}{l}16.7- \\
59.9\end{array}$ & $\begin{array}{l}60.0- \\
99.9\end{array}$ & $\begin{array}{l}100.0- \\
129.9\end{array}$ & $130.0+$ \\
\hline $\begin{array}{l}\text { Lower Wabash Unit } \\
\text { Clay } \\
\text { Oaviess } \\
\text { Gibson } \\
\text { Greene } \\
\text { Knox } \\
\text { Martin } \\
\text { Parke } \\
\text { Pike } \\
\text { Posey } \\
\text { Putnam } \\
\text { Sullivan } \\
\text { Vanderburgh } \\
\text { Vermillion } \\
\text { Vigo }\end{array}$ & $\begin{array}{r}44.3 \\
41.6 \\
42.0 \\
105.3 \\
30.8 \\
128.4 \\
87.6 \\
83.9 \\
45.1 \\
76.5 \\
64.7 \\
24.7 \\
34.7 \\
50.8 \\
\end{array}$ & $\begin{array}{l}0.1 \\
0.2 \\
0.2 \\
0.2 \\
0.2 \\
0.1 \\
0.1 \\
0.1 \\
0.2 \\
0.2 \\
0.2 \\
0.1 \\
0.1 \\
0.1 \\
\end{array}$ & $\begin{array}{l}4.2 \\
3.3 \\
3.5 \\
8.2 \\
3.4 \\
9.0 \\
5.3 \\
4.9 \\
3.4 \\
7.1 \\
4.1 \\
2.0 \\
2.6 \\
3.8 \\
\end{array}$ & $\begin{array}{l}23.2 \\
20.3 \\
21.5 \\
54.3 \\
16.2 \\
59.1 \\
44.5 \\
42.0 \\
21.9 \\
39.8 \\
32.3 \\
13.6 \\
17.9 \\
26.3 \\
\end{array}$ & $\begin{array}{r}15.2 \\
15.3 \\
14.6 \\
37.2 \\
9.5 \\
53.9 \\
34.0 \\
32.8 \\
17.3 \\
27.4 \\
24.6 \\
8.0 \\
12.1 \\
18.0 \\
\end{array}$ & $\begin{array}{l}1.6 \\
2.5 \\
2.2 \\
5.4 \\
1.5 \\
6.3 \\
3.7 \\
4.1 \\
2.3 \\
2.0 \\
3.5 \\
1.0 \\
2.0 \\
2.6 \\
\end{array}$ \\
\hline Total & 860.4 & 2.1 & 64.8 & 432.9 & 319.9 & 40.7 \\
\hline $\begin{array}{l}\text { Knobs Unit } \\
\text { Brown } \\
\text { Clark } \\
\text { Crawford } \\
\text { Oubois } \\
\text { Floyd } \\
\text { Harrison } \\
\text { Jackson } \\
\text { Lawrence } \\
\text { Monroe } \\
\text { Morgan } \\
\text { Orange } \\
\text { Owen } \\
\text { Perry } \\
\text { Scott } \\
\text { Spencer } \\
\text { Warrick } \\
\text { Washington }\end{array}$ & $\begin{array}{r}131.6 \\
84.9 \\
119.3 \\
93.0 \\
34.5 \\
132.1 \\
120.6 \\
125.3 \\
117.5 \\
86.7 \\
129.1 \\
107.5 \\
152.5 \\
43.0 \\
62.5 \\
81.4 \\
119.6\end{array}$ & $\begin{array}{l}0.3 \\
0.4 \\
0.6 \\
0.5 \\
0.2 \\
0.6 \\
0.7 \\
0.5 \\
0.4 \\
0.8 \\
1.6 \\
0.3 \\
0.9 \\
0.3 \\
0.5 \\
0.7 \\
0.4\end{array}$ & $\begin{array}{l}6.1 \\
5.7 \\
6.0 \\
6.2 \\
2.9 \\
8.4 \\
7.6 \\
5.7 \\
6.5 \\
6.3 \\
6.5 \\
9.4 \\
6.0 \\
2.9 \\
4.6 \\
7.1 \\
10.8\end{array}$ & $\begin{array}{l}53.4 \\
33.7 \\
50.2 \\
37.9 \\
13.0 \\
54.6 \\
50.5 \\
52.6 \\
49.8 \\
36.2 \\
52.8 \\
40.6 \\
58.3 \\
17.8 \\
25.7 \\
33.4 \\
43.5\end{array}$ & $\begin{array}{l}61.4 \\
37.7 \\
52.9 \\
41.5 \\
16.0 \\
60.1 \\
54.6 \\
57.2 \\
53.2 \\
38.1 \\
59.2 \\
50.1 \\
72.4 \\
19.0 \\
27.7 \\
35.0 \\
55.5\end{array}$ & $\begin{array}{r}10.4 \\
7.4 \\
9.6 \\
6.9 \\
2.4 \\
8.4 \\
7.2 \\
9.3 \\
7.6 \\
5.3 \\
9.0 \\
7.1 \\
14.9 \\
3.0 \\
4.0 \\
5.2 \\
9.4\end{array}$ \\
\hline Total & $1,741.1$ & 9.7 & 108.7 & 704.0 & 791.6 & 127.1 \\
\hline $\begin{array}{l}\text { Upland Flats Unit } \\
\text { Oearborn } \\
\text { Fayette } \\
\text { Franklin } \\
\text { Jefferson } \\
\text { Jennings } \\
\text { Ohio } \\
\text { Ripley } \\
\text { Switzerland } \\
\text { Union }\end{array}$ & $\begin{array}{l}91.1 \\
33.5 \\
80.4 \\
78.3 \\
87.6 \\
27.9 \\
75.7 \\
75.2 \\
21.4\end{array}$ & $\begin{array}{l}0.9 \\
0.6 \\
1.2 \\
0.8 \\
1.1 \\
0.3 \\
0.9 \\
0.3 \\
0.4\end{array}$ & $\begin{array}{r}19.6 \\
8.7 \\
19.5 \\
13.7 \\
19.3 \\
6.2 \\
14.0 \\
11.3 \\
5.3\end{array}$ & $\begin{array}{r}42.3 \\
14.3 \\
36.4 \\
36.9 \\
38.9 \\
12.8 \\
36.7 \\
36.1 \\
9.7\end{array}$ & $\begin{array}{r}23.4 \\
8.0 \\
18.8 \\
23.6 \\
23.3 \\
7.0 \\
21.0 \\
23.2 \\
4.9\end{array}$ & $\begin{array}{l}4.9 \\
1.9 \\
4.5 \\
3.3 \\
5.0 \\
1.6 \\
3.1 \\
4.3 \\
1.1\end{array}$ \\
\hline Total & 571.1 & 6.5 & 117.6 & 264.1 & 153.2 & 29.7 \\
\hline
\end{tabular}


(Table 15 continued)

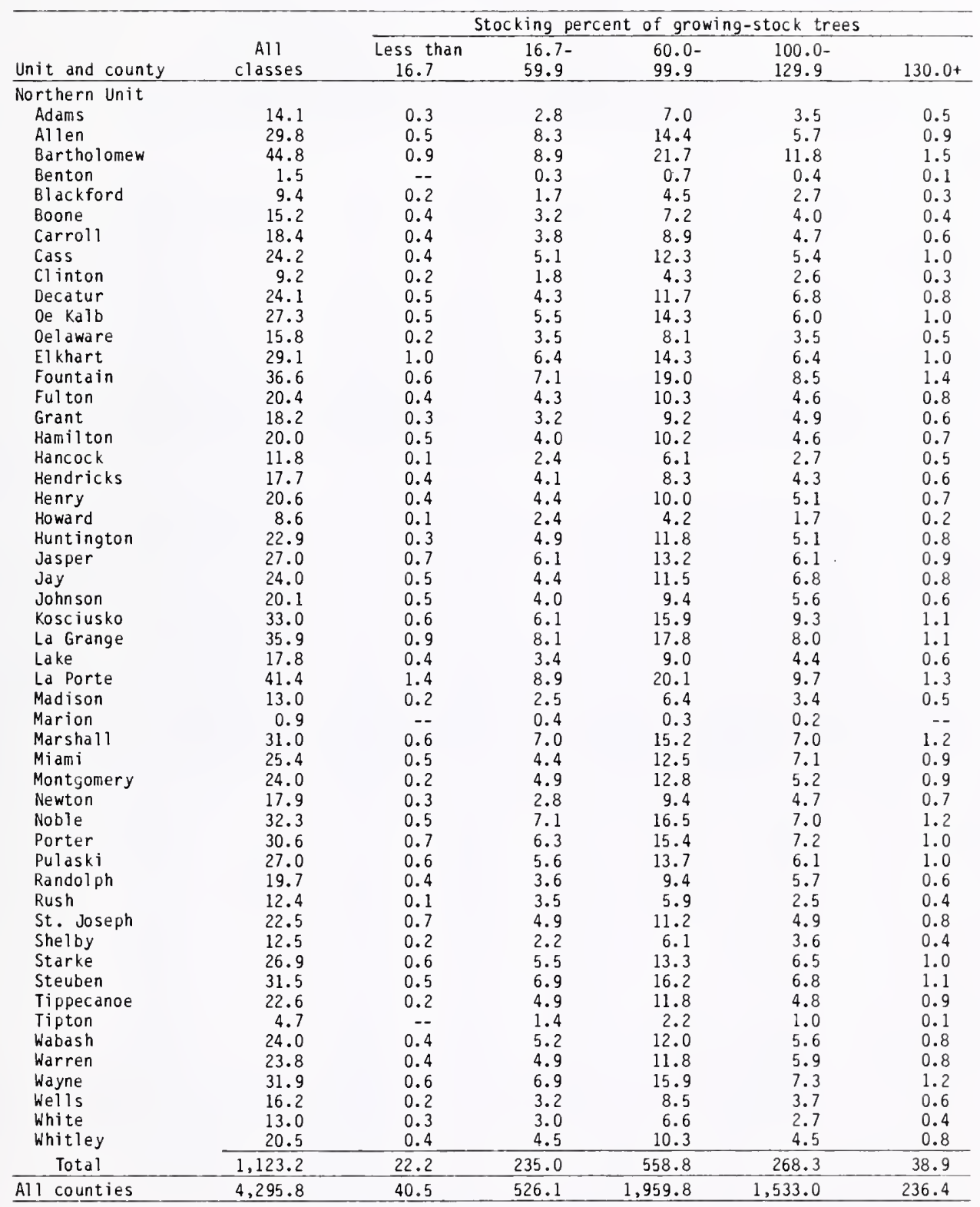


Table 16.--Area of timberland by forest type, ownership class and Forest Survey Unit, Indiana, 1986

(In thousand acres)

\begin{tabular}{|c|c|c|c|c|c|c|c|c|c|}
\hline \multirow[b]{2}{*}{ Forest type } & \multirow[b]{2}{*}{$\begin{array}{c}\text { All } \\
\text { Owners }\end{array}$} & \multicolumn{8}{|c|}{ Ownership class } \\
\hline & & $\begin{array}{c}\text { National } \\
\text { forest }\end{array}$ & $\begin{array}{c}\text { Misc. } \\
\text { federal }\end{array}$ & State & $\begin{array}{l}\text { County \& } \\
\text { municipal }\end{array}$ & $\begin{array}{c}\text { Forest } \\
\text { industry }\end{array}$ & Farmer & $\begin{array}{l}\text { Misc. } \\
\text { priv.- } \\
\text { corp. }\end{array}$ & $\begin{array}{l}\text { Misc. } \\
\text { priv.- } \\
\text { indiv. }\end{array}$ \\
\hline \multicolumn{10}{|l|}{ A1l Units } \\
\hline Jack-red-white pine & 54.7 & 11.5 & -- & 4.5 & -- & -- & 6.4 & 14.0 & 18.3 \\
\hline Shortleaf pine & 23.9 & 14.8 & -- & 3.5 & -- & -- & 1.9 & 1.6 & 2.1 \\
\hline Scotch-Virginia pine & 70.6 & -- & -- & 4.0 & -- & -- & 37.0 & 9.4 & 20.2 \\
\hline Oak-pine & 104.2 & 3.4 & 2.5 & 1.9 & -- & -- & 50.4 & 6.3 & 39.7 \\
\hline Oak-hickory & $1,370.8$ & 99.7 & 70.0 & 92.6 & 8.3 & 12.5 & 451.4 & 111.4 & 524.9 \\
\hline Chestnut-scarlet oak & 46.1 & 6.2 & -- & 13.9 & - & -- & 1.2 & -- & 24.8 \\
\hline Sassafras-persimmon & 19.8 & -- & -- & -- & -- & -- & 6.7 & 2.1 & 11.0 \\
\hline 0ak-gum & 51.7 & -- & -- & 6.6 & -- & -- & 23.0 & 6.2 & 15.9 \\
\hline Lowl and oak & 30.9 & -- & -- & -- & -- & -- & 16.2 & 4.4 & 10.3 \\
\hline Elm-ash-soft maple & 830.5 & 2.0 & 28.4 & 17.8 & 11.5 & -- & 374.7 & 103.1 & 293.0 \\
\hline Cottonwood & 18.4 & -- & $\cdots$ & 2.1 & 2.3 & -- & 2.8 & 8.8 & 2.4 \\
\hline Maple-beech & 984.7 & 10.7 & 42.1 & 13.1 & 4.8 & 3.8 & 422.1 & 88.9 & 399.2 \\
\hline Cherry-ash-yel low-poplar & 649.0 & 16.0 & 16.9 & 17.4 & 2.0 & -- & 295.2 & 48.5 & 253.0 \\
\hline Nonstocked & 40.5 & 1.7 & 2.7 & -- & -- & 2.1 & 14.9 & 2.4 & 16.7 \\
\hline All types. & $4,295.8$ & 166.0 & 162.6 & 177.4 & 28.9 & 18.4 & $1,703.9$ & 407.1 & $1,631.5$ \\
\hline \multicolumn{10}{|l|}{ Lower Wabash } \\
\hline Jack-red-white pine & 9.9 & -- & -- & -- & -- & -- & -- & 9.9 & -- \\
\hline Shortleaf pine & 3.7 & 0.5 & -- & 1.6 & -- & -- & -- & 1.6 & -- \\
\hline Scotch-Virginia pine & 17.3 & -- & -- & -- & -- & -- & 8.6 & 5.4 & 3.3 \\
\hline oak-pine & 2.3 & -- & -- & -- & -- & -- & -- & 2.3 & -- \\
\hline Oak-hickory & 296.1 & 4.5 & 40.4 & 2.0 & -- & -- & 101.7 & 25.5 & 122.0 \\
\hline Chestnut-scarlet oak & -- & -- & -- & -- & -- & -- & -- & -- & -- \\
\hline Sassafras-persimmon & 7.4 & -- & -- & -- & -- & -- & 4.8 & -- & 2.6 \\
\hline Oa k-gum & 8.1 & -- & -- & 4.3 & -- & -- & -- & 1.5 & 2.3 \\
\hline Lowl and oak & 8.3 & -- & -- & -- & -- & -- & 2.0 & 2.0 & 4.3 \\
\hline Elm-ash-soft maple & 224.4 & 0.8 & 4.6 & 11.2 & 4.0 & -- & 92.2 & 36.8 & 74.8 \\
\hline Cottonwood & 6.7 & -- & -- & 2.1 & 2.3 & -- & -- & 2.3 & -- \\
\hline Maple-beech & 159.4 & 2.2 & 8.6 & 2.0 & -- & -- & 52.8 & 24.3 & 69.5 \\
\hline Cherry-ash-yel low-poplar & 114.7 & 1.0 & 5.6 & 6.3 & 2.0 & -- & 50.4 & 11.3 & 38.1 \\
\hline Nonstocked & 2.1 & -- & -- & - & -- & 2.1 & -- & -. & -- \\
\hline Al1 types & 860.4 & 9.0 & 59.2 & 29.5 & 8.3 & 2.1 & 312.5 & 122.9 & 316.9 \\
\hline \multicolumn{10}{|l|}{ Knobs } \\
\hline Jack-red-white pine & 29.8 & 11.5 & -- & 4.5 & -- & -- & 3.6 & 4.1 & 6.1 \\
\hline Shortleaf pine & 20.2 & 14.3 & -- & 1.9 & -- & -- & 1.9 & -- & 2.1 \\
\hline Scotch-Virginia pine & 45.7 & -- & -- & 4.0 & -- & -- & 20.8 & 4.0 & 16.9 \\
\hline Oak-pine & 54.2 & 3.4 & 2.5 & 1.9 & -- & - & 25.7 & 1.9 & 18.8 \\
\hline Oak-hickory & 689.2 & 95.2 & 20.1 & 80.6 & 3.8 & 9.7 & 177.9 & 50.9 & 251.0 \\
\hline Chestnut-scarlet oak & 39.7 & 6.2 & -- & 13.9 & -- & -- & - & -- & 19.6 \\
\hline Sassafras-persimnion & 12.4 & -- & -- & -- & -. & -- & 1.9 & 2.1 & 8.4 \\
\hline Oak-эum & 25.6 & -- & -- & 2.3 & -- & -- & 13.1 & 2.0 & 8.2 \\
\hline Lowl and oak & 2.1 & -- & -- & -- & -- & -- & 2.1 & -- & -- \\
\hline Elm-ash-soft maple & 202.6 & 1.2 & 10.7 & 4.2 & -- & -- & 66.5 & 31.7 & 88.3 \\
\hline Cottonwood & 4.4 & -- & -- & -- & -- & -- & -- & 4.4 & -- \\
\hline Maple-beech & 402.2 & 8.5 & 22.6 & 10.1 & 2.1 & 3.8 & 127.7 & 38.7 & 188.7 \\
\hline Cherry-ash-yel low-poplar & 203.3 & 15.0 & 6.1 & 5.9 & -. & -. & 73.3 & 14.8 & 88.2 \\
\hline Nonstocked & 9.7 & 1.7 & -- & -- & -- & -- & -. & -- & 8.0 \\
\hline All types & $1,741.1$ & 157.0 & 62.0 & 129.3 & 5.9 & 13.5 & 514.5 & 154.6 & 704.3 \\
\hline
\end{tabular}


(Table 16 continued)

\begin{tabular}{|c|c|c|c|c|c|c|c|c|c|}
\hline \multirow[b]{2}{*}{ Unit and forest type } & \multirow[b]{2}{*}{$\begin{array}{c}\text { All } \\
\text { owners }\end{array}$} & \multicolumn{8}{|c|}{ Ownership class } \\
\hline & & $\begin{array}{c}\text { National } \\
\text { forest }\end{array}$ & $\begin{array}{l}\text { Misc. } \\
\text { federal }\end{array}$ & State & $\begin{array}{l}\text { County \& } \\
\text { municipal }\end{array}$ & $\begin{array}{l}\text { Forest } \\
\text { industry }\end{array}$ & Farmer & $\begin{array}{l}\text { Misc. } \\
\text { oriv.- } \\
\text { corp. }\end{array}$ & $\begin{array}{l}\text { Misc. } \\
\text { priv.- } \\
\text { indiv. }\end{array}$ \\
\hline \multicolumn{10}{|l|}{ Upland Flats Unit } \\
\hline Jack-red-white pine & 3.8 & - - & -- & -- & -- & $=$ & -- & -- & 3.8 \\
\hline Shortleaf pine & - & -- & -- & -- & -. & -. & - & -- & -- \\
\hline Scotch-Virginia pine & 2.0 & -- & -- & -- & -- & -. & 2.0 & -- & -. \\
\hline Oak-pine & 45.6 & -- & -- & -- & -. & -- & 22.6 & 2.1 & 20.9 \\
\hline Chestnut-scarlet oak & - & -- & -- & - & -- & - & -- & - & -- \\
\hline Sassafras-persimmon & -- & -- & $\ldots$ & -- & $\ldots$ & -- & - & - - & -- \\
\hline Oak-gum & 14.4 & - & -- & -- & -- & -- & 7.5 & 2.7 & 4.2 \\
\hline Lowland oak & 19 & -. & $\ldots$ & - & - & - & - & -- & -- \\
\hline Elm-ash-soft maple & 86.7 & -. & 2.1 & -- & - - & -- & 45.8 & 2.1 & 36.7 \\
\hline Cottonwood & -- & -- & - & -- & -- & - & -- & -- & -- \\
\hline Maple-beech & 145.2 & -- & 4.0 & -- & 2.7 & -- & 67.3 & 4.1 & 67.1 \\
\hline Cherry-ash-yel low-poplar & 150.8 & -- & -- & 2.8 & -- & -- & 76.7 & 6.6 & 64.7 \\
\hline Nonstocked & 6.5 & $\cdots$ & 2.7 & -- & -. & $\cdots$ & 1.7 & -- & 2.1 \\
\hline \multicolumn{10}{|l|}{ Northern Unit } \\
\hline Jack-red-white pine & 11.2 & - & -- & - & -- & -. & 2.8 & -- & 8.4 \\
\hline Shortleaf pine & 12 & - & -- & -- & -- & -. & -- & -- & -- \\
\hline Scotch-Virginia pine & 5.6 & - & - & -- & - & -- & 5.6 & -- & -- \\
\hline Oak-pine & 2.1 & - & -- & -- & -- & -- & 2.1 & - & -- \\
\hline Oak-hickory & 269.4 & - & 4.8 & 5.2 & 4.5 & 2.8 & 124.1 & 26.1 & 101.9 \\
\hline Chestnut-scarlet oak & 6.4 & -- & -- & -- & - & - & 1.2 & -- & 5.2 \\
\hline Sassafras-persimmon & -- & -- & -- & -- & -- & -- & - & -- & -- \\
\hline Oak-gum & 3.6 & -- & -- & -- & -- & -- & 2.4 & -- & 1.2 \\
\hline Lowland oak & 20.5 & -- & -- & -- & -- & -- & 12.1 & 2.4 & 6.0 \\
\hline Elm-ash-soft maple & 316.8 & - & 11.0 & 2.4 & 7.5 & - & 170.2 & 32.5 & 93.2 \\
\hline Cottonwood & 7.3 & -- & - & - . & -- & -- & 2.8 & 2.1 & 2.4 \\
\hline Maple-beech & 277.9 & -- & 6.9 & 1.0 & -- & -- & 174.3 & 21.8 & 73.9 \\
\hline Cherry-ash-yellow-poplar & 180.2 & -- & 5.2 & 2.4 & -- & -- & 94.8 & 15.8 & 62.0 \\
\hline Nonstocked & 22.2 & -- & -- & -- & - & -- & 13.2 & 2.4 & 6.6 \\
\hline All types & $1,123.2$ & -. & 27.9 & 11.0 & 12.0 & 2.8 & 605.6 & 103.1 & 360.8 \\
\hline
\end{tabular}


Table 17.--Area of timberland by ownership class and site class, and Forest Survey Unit, Indiana, 1986

(In thousand acres)

\begin{tabular}{|c|c|c|c|c|c|c|}
\hline \multirow[b]{2}{*}{ Unit and ownership class } & \multirow{2}{*}{$\begin{array}{c}\text { All } \\
\text { classes }\end{array}$} & \multicolumn{5}{|c|}{ Site class (cubic feet of growth per acre per year) } \\
\hline & & $16 b+$ & $120-164$ & $85-119$ & $50-84$ & $20-49$ \\
\hline $\begin{array}{l}\text { All Units } \\
\text { National forest } \\
\text { Miscellaneous federal } \\
\text { State } \\
\text { County and municipal } \\
\text { Forest industry } \\
\text { Farmer } \\
\text { Misc. private-corporation } \\
\text { Misc. private-individual }\end{array}$ & $\begin{array}{r}166.0 \\
162.6 \\
177.4 \\
28.9 \\
18.4 \\
1,703.9 \\
407.1 \\
1,631.5 \\
\end{array}$ & $\begin{array}{l}-- \\
-- \\
-- \\
-- \\
-- \\
-- \\
--\end{array}$ & $\begin{array}{r}21.7 \\
17.2 \\
27.5 \\
-- \\
3.8 \\
294.1 \\
70.0 \\
261.4\end{array}$ & $\begin{array}{r}48.1 \\
45.8 \\
44.3 \\
16.0 \\
1.9 \\
605.3 \\
139.1 \\
596.0\end{array}$ & $\begin{array}{r}58.1 \\
77.6 \\
58.3 \\
10.1 \\
5.9 \\
531.3 \\
138.6 \\
520.4 \\
\end{array}$ & $\begin{array}{r}38.1 \\
22.0 \\
47.3 \\
2.8 \\
6.8 \\
273.2 \\
59.4 \\
253.7 \\
\end{array}$ \\
\hline All owners & $4,295.8$ & -- & 695.7 & $1,496.5$ & $1,400.3$ & 703.3 \\
\hline $\begin{array}{l}\text { Lower Wabash Unit } \\
\text { National forest } \\
\text { Miscellaneous federal } \\
\text { State } \\
\text { County and municipal } \\
\text { Forest industry } \\
\text { Farmer } \\
\text { Misc. private-corporation } \\
\text { Misc. private-individual }\end{array}$ & $\begin{array}{r}9.0 \\
59.2 \\
29.5 \\
8.3 \\
2.1 \\
312.5 \\
122.9 \\
316.9 \\
\end{array}$ & $\begin{array}{l}-- \\
-- \\
-- \\
-- \\
-- \\
-- \\
--\end{array}$ & $\begin{array}{r}4.0 \\
7.0 \\
-- \\
39.9 \\
32.1 \\
57.1\end{array}$ & $\begin{array}{r}2.7 \\
16.8 \\
8.1 \\
6.0 \\
--. \\
104.5 \\
37.7 \\
111.4 \\
\end{array}$ & $\begin{array}{r}6.3 \\
30.4 \\
6.3 \\
2.3 \\
2.1 \\
126.6 \\
34.3 \\
109.3 \\
\end{array}$ & $\begin{array}{r}--. \\
8.0 \\
7.2 \\
-- \\
-- \\
41.5 \\
18.8 \\
39.1 \\
\end{array}$ \\
\hline All owners & 860.4 & -- & 141.0 & 287.2 & 317.6 & 114.6 \\
\hline $\begin{array}{l}\text { Knobs Unit } \\
\text { National forest } \\
\text { Miscellaneous federal } \\
\text { State } \\
\text { County and municipal } \\
\text { Forest industry } \\
\text { Farmer } \\
\text { Misc. private-corporation } \\
\text { Misc. private-individual }\end{array}$ & $\begin{array}{r}157.0 \\
62.0 \\
129.3 \\
5.9 \\
13.5 \\
514.5 \\
154.6 \\
704.3 \\
\end{array}$ & $\begin{array}{l}-- \\
-- \\
-- \\
-- \\
-- \\
-- \\
--\end{array}$ & $\begin{array}{r}21.7 \\
8.3 \\
14.4 \\
-- \\
3.8 \\
100.3 \\
27.0 \\
111.1\end{array}$ & $\begin{array}{r}45.4 \\
17.8 \\
30.4 \\
2.1 \\
1.9 \\
170.4 \\
48.5 \\
255.4 \\
\end{array}$ & $\begin{array}{r}51.8 \\
29.2 \\
47.2 \\
3.8 \\
3.8 \\
170.3 \\
60.0 \\
231.7\end{array}$ & $\begin{array}{r}38.1 \\
6.7 \\
37.3 \\
-- \\
4.0 \\
73.5 \\
19.1 \\
106.1 \\
\end{array}$ \\
\hline All owners & $1,741.1$ & -- & 286.6 & 571.9 & 597.8 & 284.8 \\
\hline $\begin{array}{l}\text { Upland Flats Unit } \\
\text { National forest } \\
\text { Miscellaneous federal } \\
\text { State } \\
\text { County and municipal } \\
\text { Forest industry } \\
\text { Farmer } \\
\text { Misc. private-corporation } \\
\text { Misc. private-individual }\end{array}$ & $\begin{array}{r}13.5 \\
7.6 \\
2.7 \\
-- \\
271.3 \\
26.5 \\
249.5 \\
\end{array}$ & $\begin{array}{l}-- \\
-- \\
-- \\
-- \\
-- \\
--\end{array}$ & $\begin{array}{r}-- \\
2.8 \\
-- \\
-- \\
60.4 \\
2.1 \\
41.2 \\
\end{array}$ & $\begin{array}{r}-- \\
4.0 \\
4.8 \\
2.7 \\
-.- \\
99.0 \\
9.6 \\
84.0 \\
\end{array}$ & $\begin{array}{r}-- \\
6.7 \\
-- \\
-- \\
-- \\
61.8 \\
6.1 \\
69.6\end{array}$ & $\begin{array}{r}-- \\
2.8 \\
-- \\
-- \\
-- \\
50.1 \\
8.7 \\
54.7\end{array}$ \\
\hline All owners & 571.1 & - & 106.5 & 204.1 & 144.2 & 116.3 \\
\hline $\begin{array}{l}\text { Northern Unit } \\
\text { National forest } \\
\text { Miscellaneous federal } \\
\text { State } \\
\text { County and municipal } \\
\text { Forest industry } \\
\text { Farmer } \\
\text { Misc. private-corporation } \\
\text { Misc. private-individual }\end{array}$ & $\begin{array}{r}27.9 \\
11.0 \\
12.0 \\
2.8 \\
605.6 \\
103.1 \\
360.8\end{array}$ & $\begin{array}{l}-- \\
-- \\
-- \\
-- \\
-- \\
-- \\
-- \\
--\end{array}$ & $\begin{array}{r}-- \\
4.9 \\
2.4 \\
-- \\
-- \\
93.5 \\
8.8 \\
52.0\end{array}$ & $\begin{array}{r}7 .-2 \\
1.0 \\
5.2 \\
-.- \\
231.4 \\
43.3 \\
145.2\end{array}$ & $\begin{array}{r}-- \\
11.3 \\
4.8 \\
4.0 \\
-- \\
172.6 \\
38.2 \\
109.8\end{array}$ & $\begin{array}{r}4.5 \\
2.8 \\
2.8 \\
2.8 \\
108.1 \\
12.8 \\
53.8\end{array}$ \\
\hline All owners & $1,123.2$ & -- & 161.6 & 433.3 & 340.7 & 187.6 \\
\hline
\end{tabular}


Table 18.--Area of privately owned timberland by ownership class, owner tenure, and size of holding, Indiana, 1986

(In thousand acres)

\begin{tabular}{|c|c|c|c|c|c|c|c|c|c|c|}
\hline \multirow[b]{2}{*}{$\begin{array}{l}\text { Ownership class } \\
\text { and owner tenure class }\end{array}$} & \multirow[b]{2}{*}{$\begin{array}{c}\text { All } \\
\text { sizes }\end{array}$} & \multicolumn{8}{|c|}{ Size of holding (acres) } & \multirow[b]{2}{*}{$5001+$} \\
\hline & & $1-4$ & $5-10$ & $11-20$ & $21-50$ & $51-100$ & $\begin{array}{l}101- \\
500\end{array}$ & $\begin{array}{r}501- \\
2,500 \\
\end{array}$ & $\begin{array}{l}2,501- \\
5,000\end{array}$ & \\
\hline \multicolumn{11}{|l|}{ Forest industry } \\
\hline $1-4$ years & 7.6 & -- & $\cdots$ & -- & -- & -- & 5.7 & -- & -- & 1.9 \\
\hline $5-9$ years & - & -- & -- & -- & -- & -- & -- & -- & -- & - \\
\hline $10-19$ years & 4.0 & -- & 2.1 & -- & -- & -- & -- & -- & -- & 1.9 \\
\hline $20+$ years & 6.8 & - & - & -- & - & -- & 4.7 & -- & -- & 2.1 \\
\hline All classes & 18.4 & $\ldots$ & 2.1 & -- & -- & -- & 10.4 & $\ldots$ & -- & 5.9 \\
\hline \multicolumn{11}{|l|}{ Farmer } \\
\hline 1.4 years & 173.7 & 18.2 & 3.8 & 18.4 & 33.7 & 48.5 & 46.7 & 4.4 & - & -- \\
\hline $5-9$ years & 327.7 & 14.4 & 9.9 & 65.5 & 83.2 & 69.3 & 80.6 & 4.8 & - & -. \\
\hline $10-19$ years & 529.4 & 10.5 & 36.3 & 72.1 & 181.4 & 142.8 & 82.0 & 4.3 & -- & -- \\
\hline $20+$ years & 673.1 & 26.7 & 36.4 & 95.6 & 183.0 & 174.4 & 150.4 & 6.6 & -- & -- \\
\hline All classes & $1,703.9$ & 69.8 & 86.4 & 251.6 & 481.3 & 435.0 & 359.7 & 20.1 & - & $\ldots$ \\
\hline \multicolumn{11}{|l|}{ Misc. priv.-corporation } \\
\hline $1-4$ years & 71.9 & 2.1 & -- & 2.8 & 8.5 & 8.9 & 30.3 & 11.2 & 3.5 & 4.6 \\
\hline $5-9$ years & 97.6 & 6.2 & 2.4 & 6.3 & 9.5 & 11.2 & 31.4 & 14.8 & 7.2 & 8.6 \\
\hline $10-19$ years & 118.6 & 2.4 & - & 7.1 & 8.1 & 14.0 & 24.4 & 33.4 & 3.7 & 25.5 \\
\hline $20+$ years & 119.0 & 4.3 & 4.5 & 2.4 & 14.7 & 15.1 & 21.9 & 25.8 & 7.7 & 22.6 \\
\hline All classes & 407.1 & 15.0 & 6.9 & 18.6 & 40.8 & 49.2 & 108.0 & 85.2 & 22.1 & 61.3 \\
\hline \multicolumn{11}{|l|}{ Misc. priv. -individual } \\
\hline 1.4 years & 330.9 & 29.1 & 38.1 & 37.3 & 76.8 & 68.7 & 63.7 & 8.5 & -- & 8.7 \\
\hline $5-9$ years & 368.1 & 35.6 & 18.0 & 65.9 & 125.6 & 60.3 & 61.5 & 1.2 & -- & -- \\
\hline $10-19$ years & 493.9 & 50.0 & 34.4 & 67.9 & 115.1 & 126.5 & 89.9 & 5.9 & 4.2 & - \\
\hline $20+$ years & 438.6 & 27.7 & 27.0 & 58.8 & 117.4 & 105.6 & 93.6 & 6.2 & - & 2.3 \\
\hline All classes & $1,631.5$ & 142.4 & 117.5 & 229.9 & 434.9 & 361.1 & 308.7 & 21.8 & 4.2 & 11.0 \\
\hline \multicolumn{11}{|l|}{ All private owners } \\
\hline $1-4$ years & 584.1 & 49.4 & 41.9 & 58.5 & 119.0 & 126.1 & 146.4 & 24.1 & 3.5 & 15.2 \\
\hline $5-9$ years & 793.4 & 56.2 & 30.3 & 137.7 & 218.3 & 140.8 & 173.5 & 20.8 & 7.2 & 8.6 \\
\hline 10-19 years & $1,145.9$ & 62.9 & 72.8 & 147.1 & 304.6 & 283.3 & 196.3 & 43.6 & 7.9 & 27.4 \\
\hline $20+$ years & $1,237.5$ & 58.7 & 67.9 & 156.8 & 315.1 & 295.1 & 270.6 & 38.6 & 7.7 & 27.0 \\
\hline All classes & $3,760.9$ & 227.2 & 212.9 & 500.1 & 957.0 & 845.3 & 786.8 & 127.1 & 26.3 & 78.2 \\
\hline
\end{tabular}


Table 19.--Area of timberland by ownership class and stocking class of growing-stock trees, and Forest Survey Unit, 1986

(In thousand acres)

\begin{tabular}{|c|c|c|c|c|c|c|}
\hline \multirow[b]{2}{*}{ Unit and ownership class } & \multirow[b]{2}{*}{$\begin{array}{c}\text { All } \\
\text { classes } \\
\end{array}$} & \multicolumn{5}{|c|}{ Stocking percent of growing stock trees } \\
\hline & & $\begin{array}{c}\text { Less than } \\
16.7\end{array}$ & $\begin{array}{l}16.7- \\
59.9\end{array}$ & $\begin{array}{l}60.0- \\
99.9\end{array}$ & $\begin{array}{l}100.0- \\
129.9\end{array}$ & $130.0+$ \\
\hline $\begin{array}{l}\text { All Units } \\
\text { National forest } \\
\text { Miscellaneous federal } \\
\text { State } \\
\text { County and municipal } \\
\text { Forest industry } \\
\text { Farmer } \\
\text { Misc. private-corporation } \\
\text { Misc. private-individual }\end{array}$ & $\begin{array}{r}166.0 \\
162.6 \\
177.4 \\
28.9 \\
18.4 \\
1,703.9 \\
407.1 \\
1,631.5 \\
\end{array}$ & $\begin{array}{r}1.7 \\
2.7 \\
-- \\
-- \\
2.1 \\
14.9 \\
2.4 \\
16.7\end{array}$ & $\begin{array}{r}2.0 \\
6.3 \\
6.6 \\
11.5 \\
-.- \\
266.3 \\
48.2 \\
185.2 \\
\end{array}$ & $\begin{array}{r}58.5 \\
66.2 \\
60.3 \\
1.7 \\
6.8 \\
811.0 \\
176.8 \\
778.5 \\
\end{array}$ & $\begin{array}{r}82.0 \\
76.9 \\
94.6 \\
15.7 \\
9.5 \\
526.7 \\
157.1 \\
570.5 \\
\end{array}$ & $\begin{array}{r}21.8 \\
10.5 \\
15.9 \\
-- \\
-- \\
85.0 \\
22.6 \\
80.6 \\
\end{array}$ \\
\hline A11 owners & $4,295.8$ & 40.5 & 526.1 & $1,959.8$ & $1,533.0$ & 236.4 \\
\hline $\begin{array}{l}\text { Lower Wabash Unit } \\
\text { National forest } \\
\text { Miscellaneous federal } \\
\text { State } \\
\text { County and municipal } \\
\text { Forest industry } \\
\text { Farmer } \\
\text { Misc. private-corporation } \\
\text { Misc. private-individual }\end{array}$ & $\begin{array}{r}9.0 \\
59.2 \\
29.5 \\
8.3 \\
2.1 \\
312.5 \\
122.9 \\
316.9\end{array}$ & $\begin{array}{c}-- \\
-- \\
-- \\
-- \\
2.1 \\
-- \\
-- \\
--\end{array}$ & $\begin{array}{r}1.0 \\
-. \\
4.2 \\
2.0 \\
-. \\
36.7 \\
4.3 \\
16.6\end{array}$ & $\begin{array}{r}3.0 \\
17.0 \\
15.0 \\
-. \\
-- \\
169.8 \\
58.4 \\
169.7\end{array}$ & $\begin{array}{r}5.0 \\
40.2 \\
10.3 \\
6.3 \\
-. \\
88.5 \\
54.3 \\
115.3\end{array}$ & $\begin{array}{r}-- \\
2.0 \\
-- \\
-- \\
-- \\
17.5 \\
5.9 \\
15.3\end{array}$ \\
\hline A11 owners & 860.4 & 2.1 & 64.8 & 432.9 & 319.9 & 40.7 \\
\hline $\begin{array}{l}\text { Knobs Unit } \\
\text { National forest } \\
\text { Miscellaneous federal } \\
\text { State } \\
\text { County and municipal } \\
\text { Forest industry } \\
\text { Farmer } \\
\text { Misc. private-corporation } \\
\text { Misc. private-individual }\end{array}$ & $\begin{array}{r}157.0 \\
62.0 \\
129.3 \\
5.9 \\
13.5 \\
514.5 \\
154.6 \\
704.3\end{array}$ & $\begin{array}{l}1.7 \\
-- \\
-- \\
-- \\
-- \\
-- \\
-- \\
8.0\end{array}$ & $\begin{array}{r}1.0 \\
2.5 \\
-- \\
1.7 \\
-. \\
48.8 \\
18.7 \\
36.0\end{array}$ & $\begin{array}{r}55.5 \\
33.7 \\
38.4 \\
-- \\
4.0 \\
213.0 \\
62.1 \\
297.3\end{array}$ & $\begin{array}{r}77.0 \\
20.1 \\
80.5 \\
4.2 \\
9.5 \\
221.3 \\
59.8 \\
319.2 \\
\end{array}$ & $\begin{array}{r}21.8 \\
5.7 \\
10.4 \\
-- \\
-- \\
31.4 \\
14.0 \\
43.8\end{array}$ \\
\hline A11 owners & $1,741.1$ & 9.7 & 108.7 & 704.0 & 791.6 & 127.1 \\
\hline $\begin{array}{l}\text { Upland Flats Unit } \\
\text { National forest } \\
\text { Miscellaneous federal } \\
\text { State } \\
\text { County and municipal } \\
\text { Forest industry } \\
\text { Farmer } \\
\text { Misc. private-corporation } \\
\text { Misc. private-individual }\end{array}$ & $\begin{array}{r}-. \\
13.5 \\
7.6 \\
2.7 \\
-- \\
271.3 \\
26.5 \\
249.5\end{array}$ & $\begin{array}{r}-- \\
2.7 \\
-- \\
-- \\
-- \\
1.7 \\
-- \\
2.1\end{array}$ & $\begin{array}{r}-- \\
2.1 \\
-- \\
2.7 \\
-- \\
56.3 \\
6.2 \\
50.3\end{array}$ & $\begin{array}{r}3.8 \\
2.1 \\
-- \\
-- \\
110.9 \\
10.7 \\
136.6\end{array}$ & $\begin{array}{r}-- \\
4.9 \\
-- \\
-- \\
-- \\
91.6 \\
6.9 \\
49.8\end{array}$ & $\begin{array}{r}-- \\
-- \\
5.5 \\
-. \\
-- \\
10.8 \\
2.7 \\
10.7\end{array}$ \\
\hline Al1 owners & 571.1 & 6.5 & 117.6 & 264.1 & 153.2 & 29.7 \\
\hline $\begin{array}{l}\text { Northern Unit } \\
\text { National forest } \\
\text { Miscellaneous federal } \\
\text { State } \\
\text { County and municipal } \\
\text { Forest industry } \\
\text { Farmer } \\
\text { Misc. private-corporation } \\
\text { Misc. private-individual }\end{array}$ & $\begin{array}{r}27.9 \\
11.0 \\
12.0 \\
2.8 \\
605.6 \\
103.1 \\
360.8\end{array}$ & $\begin{array}{r}-- \\
-- \\
- \\
-- \\
-- \\
13.2 \\
2.4 \\
6.6\end{array}$ & $\begin{array}{r}1.7 \\
2.4 \\
5.1 \\
--. \\
124.5 \\
19.0 \\
82.3\end{array}$ & $\begin{array}{r}11 . \overline{7} \\
4.8 \\
1.7 \\
2.8 \\
317.3 \\
45.6 \\
174.9\end{array}$ & $\begin{array}{r}11 . \overline{-} \\
3.8 \\
5.2 \\
-- \\
125.3 \\
36.1 \\
86.2\end{array}$ & $\begin{array}{r}-- \\
2.8 \\
-- \\
-- \\
-- \\
25.3 \\
-- \\
10.8\end{array}$ \\
\hline All owners & $1,123.2$ & 22.2 & 235.0 & 558.8 & 268.3 & 38.9 \\
\hline
\end{tabular}


Table 20.--Area of timberland by forest type and stand-size class, and Forest Survey Unit, 1986

(In thousand acres)

\begin{tabular}{|c|c|c|c|c|c|}
\hline \multirow[b]{2}{*}{ Unit and forest type } & \multirow[b]{2}{*}{$\begin{array}{c}\text { All } \\
\text { stands }\end{array}$} & \multicolumn{4}{|c|}{ Stand-size class } \\
\hline & & Sawtimber & Poletimber & $\begin{array}{l}\text { Sapling \& } \\
\text { seedling }\end{array}$ & Nonstocked \\
\hline $\begin{array}{l}\text { All Units } \\
\text { Jack-red-white pine } \\
\text { Shortleaf pine } \\
\text { Scotch-Virginia pine } \\
\text { Oak-pine } \\
\text { Oak-hickory } \\
\text { Chestnut-scarlet oak } \\
\text { Sassafras-persimmon } \\
\text { Oak-gum } \\
\text { Lowland oak } \\
\text { Elm-ash-soft maple } \\
\text { Cottonwood } \\
\text { Maple-beech } \\
\text { Cherry-ash-yellow-poplar } \\
\text { Nonstocked }\end{array}$ & $\begin{array}{r}54.7 \\
23.9 \\
70.6 \\
104.2 \\
1,370.8 \\
46.1 \\
19.8 \\
51.7 \\
30.9 \\
830.5 \\
18.4 \\
984.7 \\
649.0 \\
40.5 \\
\end{array}$ & $\begin{array}{r}22.3 \\
7.6 \\
24.4 \\
44.6 \\
975.9 \\
46.1 \\
--. \\
29.7 \\
25.3 \\
495.4 \\
11.7 \\
692.3 \\
394.7 \\
--\end{array}$ & $\begin{array}{r}18.3 \\
14.5 \\
14.1 \\
25.8 \\
189.8 \\
-- \\
4.2 \\
4.6 \\
3.6 \\
169.1 \\
6.7 \\
110.5 \\
112.3 \\
-- \\
\end{array}$ & $\begin{array}{r}14.1 \\
1.8 \\
32.1 \\
33.8 \\
205.1 \\
-- \\
15.6 \\
17.4 \\
2.0 \\
166.0 \\
-- \\
181.9 \\
142.0 \\
-- \\
\end{array}$ & $\begin{array}{l}-- \\
-- \\
-- \\
-- \\
-- \\
-- \\
-- \\
-- \\
-- \\
-- \\
-- \\
-- \\
-- \\
40.5 \\
\end{array}$ \\
\hline All types & $4,295.8$ & $2,770.0$ & 673.5 & 811.8 & 40.5 \\
\hline $\begin{array}{l}\text { Lower wabash Unit } \\
\text { Jack-red-white pine } \\
\text { Shortleaf pine } \\
\text { Scotch-Virginia pine } \\
\text { Oak-pine } \\
\text { Oak-hickory } \\
\text { Chestnut-scarlet oak } \\
\text { Sassafras-persimmon } \\
\text { Oak-gum } \\
\text { Lowland oak } \\
\text { Elm-ash-soft maple } \\
\text { Cottonwood } \\
\text { Maple-beech } \\
\text { Cherry-ash-yellow-poplar } \\
\text { Nonstocked }\end{array}$ & $\begin{array}{r}9.9 \\
3.7 \\
17.3 \\
2.3 \\
296.1 \\
-- \\
7.4 \\
8.1 \\
8.3 \\
224.4 \\
6.7 \\
159.4 \\
114.7 \\
2.1 \\
\end{array}$ & $\begin{array}{r}7.6 \\
-- \\
7.4 \\
2.3 \\
218.6 \\
-- \\
-- \\
5.8 \\
6.3 \\
132.5 \\
2.3 \\
113.1 \\
86.6 \\
-- \\
\end{array}$ & $\begin{array}{r}2.3 \\
3.7 \\
3.1 \\
-- \\
27.4 \\
-- \\
-- \\
2.3 \\
-- \\
36.1 \\
4.4 \\
20.5 \\
12.2 \\
-- \\
\end{array}$ & $\begin{array}{r}-- \\
-- \\
6.8 \\
-- \\
50.1 \\
-- \\
7.4 \\
-- \\
2.0 \\
55.8 \\
-- \\
25.8 \\
15.9 \\
--\end{array}$ & $\begin{array}{l}-- \\
-- \\
-- \\
-- \\
-- \\
-- \\
-- \\
-- \\
-- \\
-- \\
-- \\
-- \\
-- \\
2.1 \\
\end{array}$ \\
\hline A11 types & 860.4 & 582.5 & 112.0 & 163.8 & 2.1 \\
\hline $\begin{array}{l}\text { Knobs Unit } \\
\text { Jack-red-white pine } \\
\text { Shortleaf pine } \\
\text { Scotich-Virginia pine } \\
\text { Oak-pine } \\
\text { Oak-hickory } \\
\text { Chestnut-scarlet oak } \\
\text { Sassafras-persimmon } \\
\text { Oak-gum } \\
\text { Lowland oak } \\
\text { Elm-ash-soft maple } \\
\text { Cottonwood } \\
\text { Maple-beech } \\
\text { Cherry-ash-yellow-poplar } \\
\text { Nonstocked }\end{array}$ & $\begin{array}{r}29.8 \\
20.2 \\
45.7 \\
54.2 \\
689.2 \\
39.7 \\
12.4 \\
25.6 \\
2.1 \\
202.6 \\
4.4 \\
402.2 \\
203.3 \\
9.7 \\
\end{array}$ & $\begin{array}{r}8.1 \\
7.6 \\
14.2 \\
25.3 \\
502.5 \\
39.7 \\
-.- \\
16.7 \\
2.1 \\
105.1 \\
2.1 \\
295.9 \\
129.4 \\
-.-\end{array}$ & $\begin{array}{r}7.6 \\
10.8 \\
8.2 \\
13.3 \\
86.8 \\
-- \\
4.2 \\
2.3 \\
-- \\
48.3 \\
2.3 \\
38.6 \\
31.6 \\
--. \\
\end{array}$ & $\begin{array}{r}14.1 \\
1.8 \\
23.3 \\
15.6 \\
99.9 \\
-- \\
8.2 \\
6.6 \\
-- \\
49.2 \\
-- \\
67.7 \\
42.3 \\
-.-\end{array}$ & $\begin{array}{l}-- \\
-- \\
-- \\
-- \\
-- \\
-- \\
-- \\
-- \\
-- \\
-- \\
-- \\
-- \\
-- \\
9.7 \\
\end{array}$ \\
\hline All types & $1,741.1$ & $1,148.7$ & 254.0 & 328.7 & 9.7 \\
\hline
\end{tabular}


(Table 20 continued)

\begin{tabular}{|c|c|c|c|c|c|}
\hline \multirow[b]{2}{*}{ Unit and forest type } & \multirow[b]{2}{*}{$\begin{array}{l}\text { All } \\
\text { stands }\end{array}$} & \multicolumn{4}{|c|}{ Stand-size class } \\
\hline & & Sawtimber & Poletimber & $\begin{array}{l}\text { Sapling \& } \\
\text { seedling }\end{array}$ & Nonstocked \\
\hline $\begin{array}{l}\text { Upland Flats Unit } \\
\text { Jack-red-white pine } \\
\text { Shortleaf pine } \\
\text { Scotch-Virginia pine } \\
\text { Oak-pine } \\
\text { Oak-hickory } \\
\text { Chestnut-scarlet oak } \\
\text { Sassafras-persimmon } \\
\text { Oak-gum } \\
\text { Lowland oak } \\
\text { Elm-ash-soft maple } \\
\text { Cottonwood } \\
\text { Maple-beech } \\
\text { Cherry-ash-yellow-poplar } \\
\text { Nonstocked }\end{array}$ & $\begin{array}{r}3.8 \\
-- \\
2.0 \\
45.6 \\
116.1 \\
-- \\
-- \\
14.4 \\
-- \\
86.7 \\
-- \\
145.2 \\
150.8 \\
6.5 \\
\end{array}$ & $\begin{array}{r}3.8 \\
-- \\
-- \\
17.0 \\
70.9 \\
-- \\
-- \\
4.8 \\
-- \\
44.4 \\
-- \\
83.1 \\
61.4 \\
-- \\
\end{array}$ & $\begin{array}{r}-- \\
-- \\
-- \\
12.5 \\
17.4 \\
\ldots \\
-- \\
-- \\
-- \\
21.8 \\
-- \\
16.9 \\
45.3 \\
--\end{array}$ & $\begin{array}{r}-. \\
-. \\
2.0 \\
16.1 \\
27.8 \\
-- \\
-- \\
9.6 \\
-- \\
20.5 \\
-- \\
45.2 \\
44.1 \\
--\end{array}$ & $\begin{array}{l}-- \\
-- \\
-- \\
-- \\
-- \\
- \\
-- \\
-- \\
- \\
-- \\
-- \\
-- \\
-- \\
6.5\end{array}$ \\
\hline All types & 571.1 & 285.4 & 113.9 & 165.3 & 6.5 \\
\hline $\begin{array}{l}\text { Northern Unit } \\
\text { Jack-red-white pine } \\
\text { Shortleaf pine } \\
\text { Scotch-Virginia pine } \\
\text { Oak-pine } \\
\text { Oak-hickory } \\
\text { Chestnut-scarlet oak } \\
\text { Sassafras-persimmon } \\
\text { Oak-gum } \\
\text { Lowland oak } \\
\text { Elm-ash-soft maple } \\
\text { Cottonwood } \\
\text { Maple-beech } \\
\text { Cherry-ash-yellow-poplar } \\
\text { Nonstocked }\end{array}$ & $\begin{array}{r}11.2 \\
-.- \\
5.6 \\
2.1 \\
269.4 \\
6.4 \\
-. \\
3.6 \\
20.5 \\
316.8 \\
7.3 \\
277.9 \\
180.2 \\
22.2\end{array}$ & $\begin{array}{r}2.8 \\
-. \\
2.8 \\
-. \\
183.9 \\
6.4 \\
-- \\
2.4 \\
16.9 \\
213.4 \\
7.3 \\
200.2 \\
117.3 \\
.-\end{array}$ & $\begin{array}{r}8.4 \\
-. \\
2.8 \\
-- \\
58.2 \\
-. \\
-- \\
-- \\
3.6 \\
62.9 \\
-- \\
34.5 \\
23.2 \\
--\end{array}$ & $\begin{array}{r}-- \\
-- \\
-- \\
2.1 \\
27.3 \\
-- \\
-- \\
1.2 \\
-- \\
40.5 \\
-- \\
43.2 \\
39.7 \\
-.\end{array}$ & $\begin{array}{c}-- \\
- \\
- \\
-- \\
-- \\
-- \\
-- \\
-- \\
-- \\
-- \\
-- \\
-- \\
-- \\
22.2\end{array}$ \\
\hline All types & $1,123.2$ & 753.4 & 193.6 & 154.0 & 22.2 \\
\hline
\end{tabular}


Table 21.--Area of timberland by ownership class and stand-volume class, and Forest Survey Unit, 1986

(1n thousand acres)

\begin{tabular}{|c|c|c|c|c|}
\hline \multirow[b]{2}{*}{ Unit and ownership class } & \multirow[b]{2}{*}{$\begin{array}{c}\text { All } \\
\text { classes } \\
\end{array}$} & \multicolumn{3}{|c|}{ Stand-volume class (board feet $-1 /$ ) } \\
\hline & & $\begin{array}{c}\text { Less than } \\
1,500\end{array}$ & $\begin{array}{c}1,500 \text { to } \\
5,000\end{array}$ & $5,000+$ \\
\hline $\begin{array}{l}\text { All Units } \\
\text { National forest } \\
\text { Miscellaneous federal } \\
\text { State } \\
\text { County and municipal } \\
\text { Forest industry } \\
\text { Farmer } \\
\text { Misc. private-corporation } \\
\text { Misc. private-individual }\end{array}$ & $\begin{array}{r}166.0 \\
162.6 \\
177.4 \\
28.9 \\
18.4 \\
1,703.9 \\
407.1 \\
1,631.5 \\
\end{array}$ & $\begin{array}{r}34.2 \\
47.7 \\
34.2 \\
6.4 \\
2.1 \\
552.7 \\
143.4 \\
527.7 \\
\end{array}$ & $\begin{array}{r}52.4 \\
49.0 \\
38.2 \\
15.0 \\
6.6 \\
563.0 \\
131.6 \\
507.7 \\
\end{array}$ & $\begin{array}{r}79.4 \\
65.9 \\
105.0 \\
7.5 \\
9.7 \\
588.2 \\
132.1 \\
596.1 \\
\end{array}$ \\
\hline All owners & $4,295.8$ & $1,348.4$ & $1,363.5$ & $1,583.9$ \\
\hline $\begin{array}{l}\text { Lower Wabash Unit } \\
\text { National forest } \\
\text { Miscellaneous federal } \\
\text { State } \\
\text { County and municipal } \\
\text { Forest industry } \\
\text { Farmer } \\
\text { Misc. private-corporation } \\
\text { Misc. private-individual }\end{array}$ & $\begin{array}{r}9.0 \\
59.2 \\
29.5 \\
8.3 \\
2.1 \\
312.5 \\
122.9 \\
316.9 \\
\end{array}$ & $\begin{array}{r}1.0 \\
12.8 \\
10.9 \\
2.0 \\
2.1 \\
88.9 \\
40.7 \\
89.7\end{array}$ & $\begin{array}{r}0.5 \\
18.4 \\
4.3 \\
4.0 \\
112.2 \\
49.7 \\
95.9\end{array}$ & $\begin{array}{r}7.5 \\
28.0 \\
14.3 \\
2.3 \\
111.4 \\
32.5 \\
131.3\end{array}$ \\
\hline All owners & 860.4 & 248.1 & 285.0 & 327.3 \\
\hline $\begin{array}{l}\text { Knobs Unit } \\
\text { National forest } \\
\text { Miscellaneous federal } \\
\text { State } \\
\text { County and municipal } \\
\text { Forest industry } \\
\text { Farmer } \\
\text { Misc. private-corporation } \\
\text { Misc. private-individual }\end{array}$ & $\begin{array}{r}157.0 \\
62.0 \\
129.3 \\
5.9 \\
13.5 \\
514.5 \\
154.6 \\
704.3 \\
\end{array}$ & $\begin{array}{r}33.2 \\
20.1 \\
18.1 \\
-- \\
-- \\
147.4 \\
52.2 \\
180.0 \\
\end{array}$ & $\begin{array}{r}51.9 \\
14.4 \\
31.5 \\
5.9 \\
3.8 \\
165.4 \\
37.7 \\
229.6 \\
\end{array}$ & $\begin{array}{r}71.9 \\
27.5 \\
79.7 \\
-.- \\
9.7 \\
201.7 \\
64.7 \\
294.7 \\
\end{array}$ \\
\hline Al1 owners & $1,741.1$ & 451.0 & 540.2 & 749.9 \\
\hline $\begin{array}{l}\text { Upland Flats Unit } \\
\text { National forest } \\
\text { Miscellaneous federal } \\
\text { State } \\
\text { County and municipal } \\
\text { Forest industry } \\
\text { Farmer } \\
\text { Misc. private-corporation } \\
\text { Misc. private-individual }\end{array}$ & $\begin{array}{r}-- \\
13.5 \\
7.6 \\
2.7 \\
--- \\
271.3 \\
26.5 \\
249.5 \\
\end{array}$ & $\begin{array}{r}10 . \overline{7} \\
-- \\
2.7 \\
-\overline{-} \\
121.4 \\
15.3 \\
138.2 \\
\end{array}$ & $\begin{array}{r}-- \\
-- \\
-- \\
-- \\
-- \\
62.3 \\
2.1 \\
45.2 \\
\end{array}$ & $\begin{array}{r}-- \\
2.8 \\
7.6 \\
-- \\
-.- \\
87.6 \\
9.1 \\
66.1 \\
\end{array}$ \\
\hline All owners & 571.1 & 288.3 & 109.6 & 173.2 \\
\hline $\begin{array}{l}\text { Northern Unit } \\
\text { National forest } \\
\text { Miscellaneous federal } \\
\text { State } \\
\text { County and municipal } \\
\text { Forest industry } \\
\text { Farmer } \\
\text { Misc. private-corporation } \\
\text { Misc. private-individual }\end{array}$ & $\begin{array}{r}-- \\
27.9 \\
11.0 \\
12.0 \\
2.8 \\
605.6 \\
103.1 \\
360.8 \\
\end{array}$ & $\begin{array}{r}4.1 \\
5.2 \\
1.7 \\
-- \\
195.0 \\
35.2 \\
119.8 \\
\end{array}$ & $\begin{array}{r}16.2 \\
2.4 \\
5.1 \\
2.8 \\
223.1 \\
42.1 \\
137.0 \\
\end{array}$ & $\begin{array}{r}7.6 \\
3.4 \\
5.2 \\
-.- \\
187.5 \\
25.8 \\
104.0\end{array}$ \\
\hline All owners & $1,123.2$ & 361.0 & 428.7 & 333.5 \\
\hline
\end{tabular}

1/International $1 / 4$-inch rule. 
Table 22.--Area of timberland by forest type, stand-size class, and ownership class, Indiana, 1986

(In thousand acres)

\begin{tabular}{|c|c|c|c|c|c|c|c|c|c|}
\hline \multirow[b]{2}{*}{$\begin{array}{l}\text { Forest type and } \\
\text { stand-size class }\end{array}$} & \multirow[b]{2}{*}{$\begin{array}{c}\text { All } \\
\text { owners }\end{array}$} & \multicolumn{8}{|c|}{ Ownership class } \\
\hline & & $\begin{array}{l}\text { National } \\
\text { forest }\end{array}$ & $\begin{array}{l}\text { Misc. } \\
\text { federal }\end{array}$ & State & $\begin{array}{l}\text { County } \& \\
\text { municipal }\end{array}$ & $\begin{array}{l}\text { Forest } \\
\text { industry }\end{array}$ & Farmer & $\begin{array}{l}\text { Misc. } \\
\text { priv.- } \\
\text { Corp. }\end{array}$ & $\begin{array}{l}\text { Misc. } \\
\text { priv.- } \\
\text { indiv. }\end{array}$ \\
\hline $\begin{array}{l}\text { Jack-red-white-pine } \\
\text { Sawtimber } \\
\text { Poletimber } \\
\text { Sapling \& seedling }\end{array}$ & $\begin{array}{l}22.3 \\
18.3 \\
14.1 \\
\end{array}$ & $\begin{array}{r}4.1 \\
-- \\
7.4\end{array}$ & $\begin{array}{l}-- \\
-- \\
--\end{array}$ & $\begin{array}{r}1.9 \\
-- \\
2.6\end{array}$ & $\begin{array}{l}-- \\
-- \\
--\end{array}$ & $\begin{array}{l}-- \\
-- \\
--\end{array}$ & $\begin{array}{r}2.8 \\
3.6 \\
-- \\
\end{array}$ & $\begin{array}{l}9.7 \\
2.3 \\
2.0 \\
\end{array}$ & $\begin{array}{r}3.8 \\
12.4 \\
2.1 \\
\end{array}$ \\
\hline All stands & 54.7 & 11.5 & -- & 4.5 & -- & -- & 6.4 & 14.0 & 18.3 \\
\hline $\begin{array}{l}\text { Shortleaf pine } \\
\text { Sawtimber } \\
\text { Poletimber } \\
\text { Sapling \& seedling }\end{array}$ & $\begin{array}{r}7.6 \\
14.5 \\
1.8 \\
\end{array}$ & $\begin{array}{r}1.7 \\
11.3 \\
1.8 \\
\end{array}$ & $\begin{array}{l}-- \\
-- \\
--\end{array}$ & $\begin{array}{l}1.9 \\
1.6 \\
-\because \\
\end{array}$ & $\begin{array}{l}-- \\
-- \\
-\end{array}$ & -- & $\begin{array}{r}1.9 \\
-- \\
-- \\
\end{array}$ & $\begin{array}{r}-- \\
1.6 \\
--\end{array}$ & $\begin{array}{r}2.1 \\
=- \\
-- \\
\end{array}$ \\
\hline All stands & 23.9 & 14.8 & $=$ & 3.5 & -- & -- & 1.9 & 1.6 & 2.1 \\
\hline $\begin{array}{l}\text { Scotch-Virginia pine } \\
\text { Sawtimber } \\
\text { Poletimber } \\
\text { Sapling \& seedling }\end{array}$ & $\begin{array}{l}24.4 \\
14.1 \\
32.1 \\
\end{array}$ & $\begin{array}{l}-- \\
-- \\
--\end{array}$ & $\begin{array}{l}-- \\
-- \\
--\end{array}$ & $\begin{array}{r}4.0 \\
-- \\
-- \\
\end{array}$ & -- & $\begin{array}{l}-- \\
-- \\
-\end{array}$ & $\begin{array}{r}9.7 \\
6.8 \\
20.5 \\
\end{array}$ & $\begin{array}{l}4.2 \\
3.1 \\
2.1 \\
\end{array}$ & $\begin{array}{l}6.5 \\
4.2 \\
9.5 \\
\end{array}$ \\
\hline All stands & 70.6 & -- & -- & 4.0 & -- & -- & 37.0 & 9.4 & 20.2 \\
\hline $\begin{array}{l}\text { Oak-pine } \\
\text { Sawtimber } \\
\text { Poletimber } \\
\text { Sapling \& seedling }\end{array}$ & $\begin{array}{l}44.6 \\
25.8 \\
33.8 \\
\end{array}$ & $\begin{array}{r}3.4 \\
-- \\
--\end{array}$ & $\begin{array}{r}-- \\
2.5 \\
--\end{array}$ & $\begin{array}{r}1.9 \\
-- \\
-- \\
\end{array}$ & +- & $\begin{array}{l}-- \\
-- \\
--\end{array}$ & $\begin{array}{l}20.6 \\
12.1 \\
17.7\end{array}$ & $\begin{array}{r}4.2 \\
2.1 \\
\end{array}$ & $\begin{array}{l}14.5 \\
11.2 \\
14.0\end{array}$ \\
\hline All stands & 104.2 & 3.4 & 2.5 & 1.9 & -- & $\cdots$ & 50.4 & 6.3 & 39.7 \\
\hline $\begin{array}{l}\text { Oak-hickory } \\
\text { Sawtimber } \\
\text { Poletimber } \\
\text { Sapling \& seedling }\end{array}$ & $\begin{array}{l}975.9 \\
189.8 \\
205.1 \\
\end{array}$ & $\begin{array}{r}73.4 \\
17.3 \\
9.0 \\
\end{array}$ & $\begin{array}{r}53.5 \\
8.5 \\
8.0 \\
\end{array}$ & $\begin{array}{r}76.4 \\
9.2 \\
7.0 \\
\end{array}$ & $\begin{array}{r}6.6 \\
-\overline{1.7} \\
\end{array}$ & $\begin{array}{r}10.6 \\
1.9 \\
-. \\
\end{array}$ & $\begin{array}{r}316.7 \\
69.0 \\
65.7 \\
\end{array}$ & $\begin{array}{l}79.7 \\
10.1 \\
21.6 \\
\end{array}$ & $\begin{array}{r}359.0 \\
73.8 \\
92.1 \\
\end{array}$ \\
\hline All stands & $1,370.8$ & 99.7 & 70.0 & 92.6 & 8.3 & 12.5 & 451.4 & 111.4 & 524.9 \\
\hline $\begin{array}{l}\text { Chestnut-scarlet oak } \\
\text { Sawtimber } \\
\text { Poletimber } \\
\text { Sapling \& seedling }\end{array}$ & $\begin{array}{r}46.1 \\
-- \\
-- \\
\end{array}$ & $\begin{array}{r}6.2 \\
-- \\
--\end{array}$ & $\begin{array}{l}-- \\
-- \\
--\end{array}$ & $\begin{array}{r}13.9 \\
-- \\
-- \\
\end{array}$ & $\begin{array}{l}-- \\
-- \\
--\end{array}$ & $\begin{array}{l}-- \\
-- \\
--\end{array}$ & $\begin{array}{r}1.2 \\
-- \\
--\end{array}$ & $\begin{array}{l}-- \\
-- \\
--\end{array}$ & $\begin{array}{r}24.8 \\
-- \\
-- \\
\end{array}$ \\
\hline All stands & 46.1 & 6.2 & -- & 13.9 & -- & $\cdots$ & 1.2 & -- & 24.8 \\
\hline $\begin{array}{l}\text { Sassafras-persimmon } \\
\text { Sawtimber } \\
\text { Poletimber } \\
\text { Sapl ing \& seedling }\end{array}$ & $\begin{array}{r}4.2 \\
15.6 \\
\end{array}$ & -- & -- & $\begin{array}{l}-- \\
-- \\
--\end{array}$ & $\begin{array}{l}-- \\
-- \\
--\end{array}$ & $\begin{array}{l}-- \\
-- \\
--\end{array}$ & 6.7 & $\begin{array}{l}-- \\
-- \\
2.1\end{array}$ & $\begin{array}{l}-- \\
4.2 \\
6.8 \\
\end{array}$ \\
\hline All stands & 19.8 & -- & -- & -- & -- & -- & 6.7 & 2.1 & 11.0 \\
\hline $\begin{array}{l}\text { Oak-gum } \\
\text { Sawtimber } \\
\text { Poletimber } \\
\text { Sapling \& seedling }\end{array}$ & $\begin{array}{r}29.7 \\
4.6 \\
17.4 \\
\end{array}$ & $\begin{array}{l}-- \\
-- \\
--\end{array}$ & $\begin{array}{l}-- \\
-- \\
-- \\
\end{array}$ & $\begin{array}{r}4.3 \\
2.3 \\
-- \\
\end{array}$ & $\begin{array}{l}-- \\
-- \\
-- \\
\end{array}$ & $\begin{array}{l}-- \\
-- \\
\end{array}$ & $\begin{array}{r}17.8 \\
-- \\
5.2\end{array}$ & $\begin{array}{r}1.5 \\
-- \\
4.7 \\
\end{array}$ & $\begin{array}{l}6.1 \\
2.3 \\
7.5 \\
\end{array}$ \\
\hline All stands & 51.7 & -- & -- & 6.6 & -- & -- & 23.0 & 6.2 & 15.9 \\
\hline
\end{tabular}

(Table 22 continued on next page) 


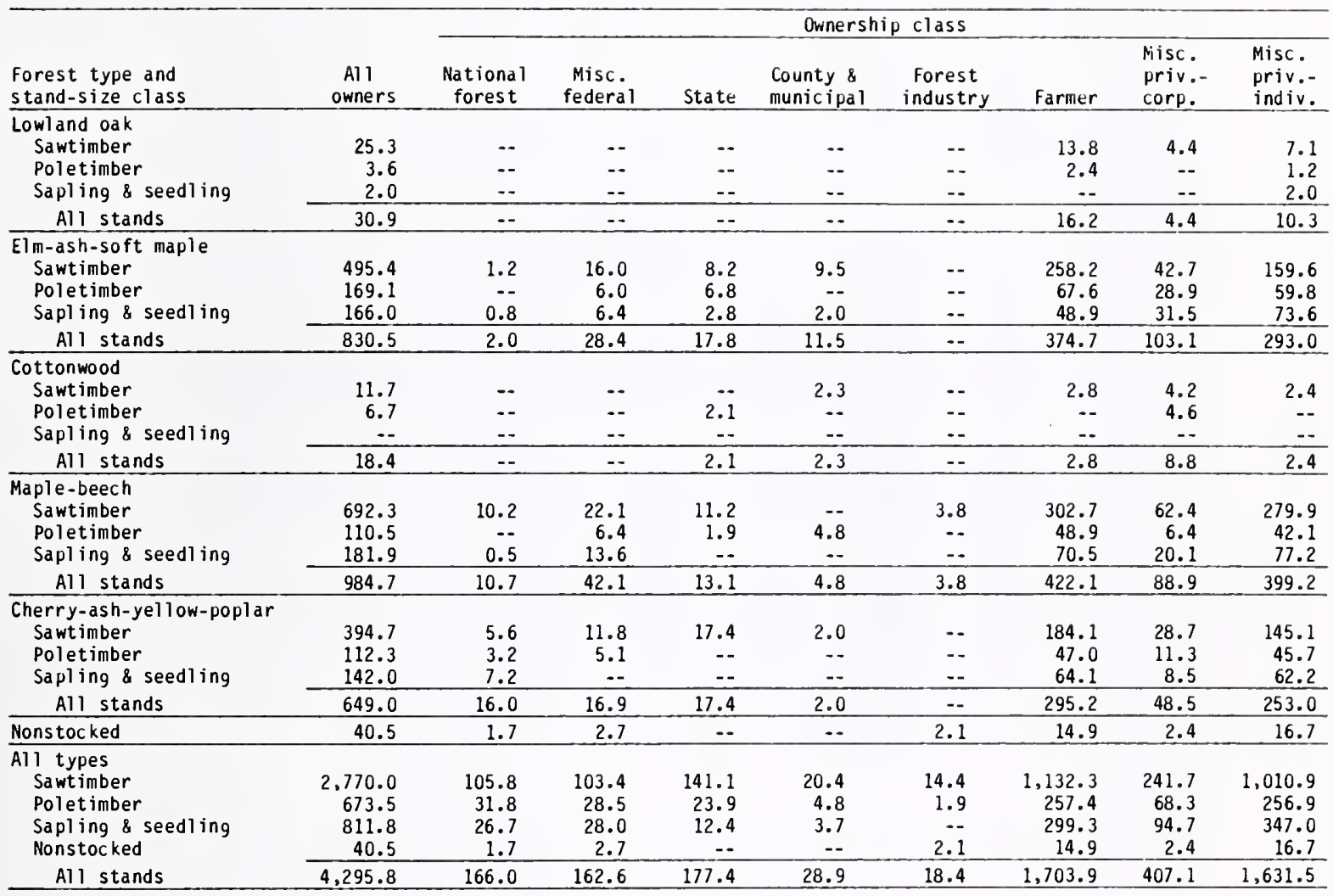


Table 23.--Area of tumberland by forest type, stand-size class, and site class, Indiana, lybo

(In thousand acres)

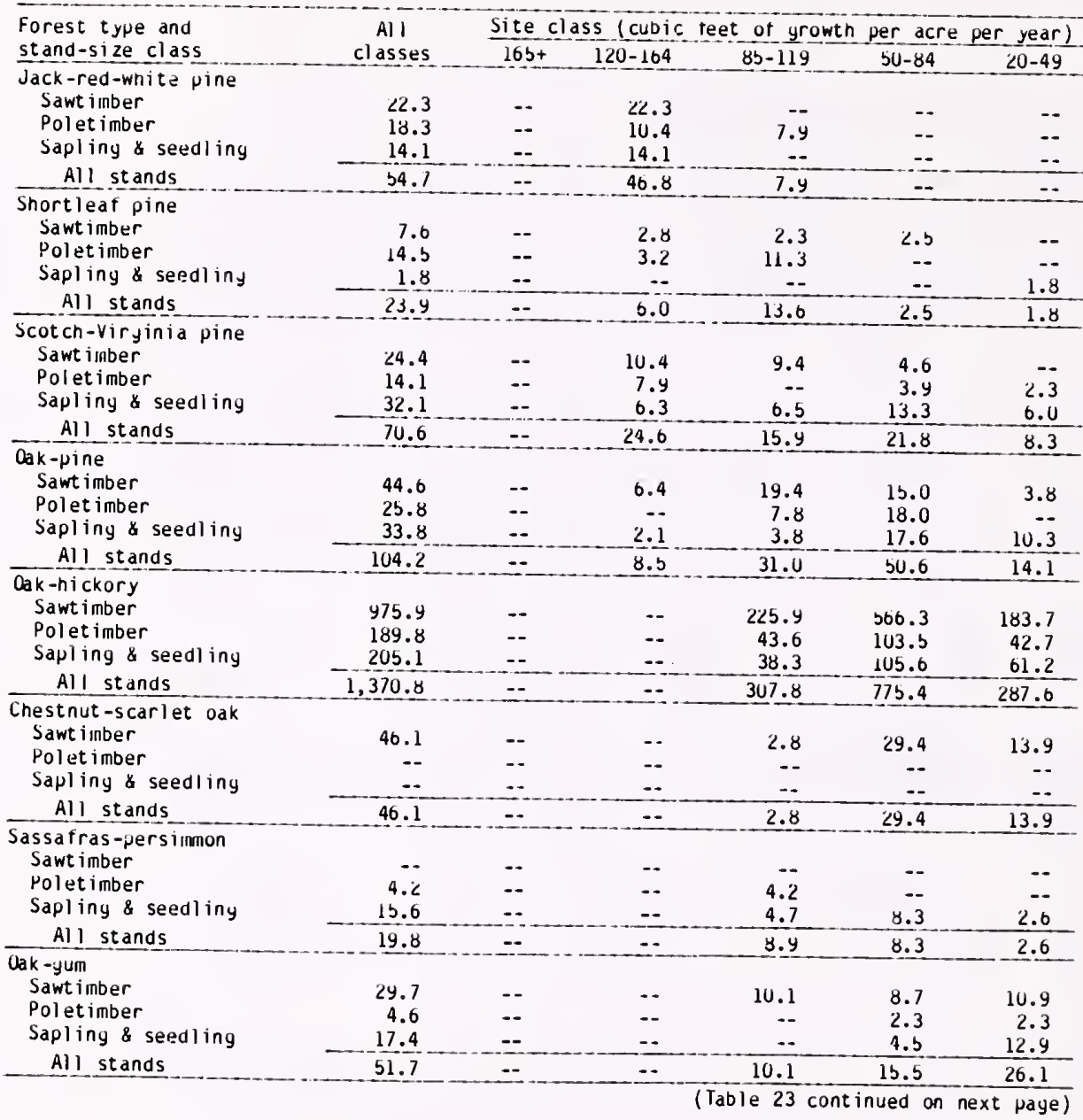


(Table 23 continued)

\begin{tabular}{|c|c|c|c|c|c|c|}
\hline \multirow{2}{*}{$\begin{array}{l}\text { Forest type and } \\
\text { stand-size class }\end{array}$} & \multirow{2}{*}{$\begin{array}{c}\text { All } \\
\text { classes }\end{array}$} & \multicolumn{2}{|c|}{ Site class (cubic } & \multicolumn{3}{|c|}{ c feet of yrowth per acre per year) } \\
\hline & & $165+$ & $120-164$ & $85-119$ & $50-84$ & $20-49$ \\
\hline \multicolumn{7}{|l|}{ Lowl and oak } \\
\hline Sawtimber & 25.3 & -- & -- & 7.1 & 11.3 & 6.4 \\
\hline Poletimber & 3.6 & -- & -- & - & 1.2 & 2.4 \\
\hline Sapling \& seedling & 2.0 & -- & -- & -- & -- & 2.0 \\
\hline All st ands & 30.9 & $=$ & $=$ & 7.1 & 12.5 & 11.3 \\
\hline \multicolumn{7}{|l|}{ Elm-ash-soft maple } \\
\hline Sawt imber & 495.4 & -- & -- & 200.1 & 207.6 & 87.7 \\
\hline Poletimber & 169.1 & -- & -- & 66.3 & 53.5 & 49.3 \\
\hline Sapling \& seedling & 166.0 & -- & -- & 38.6 & 40.4 & 87.0 \\
\hline All stands & 830.5 & -- & - & 305.0 & 301.5 & 224.0 \\
\hline \multicolumn{7}{|l|}{ Cottonwood } \\
\hline Sawtimber & 11.7 & -- & -- & 2.8 & 6.8 & 2.1 \\
\hline Poletimber & 6.7 & -- & -- & -- & -- & 6.7 \\
\hline Sapling \& seedling & $=$ & $-=$ & -- & -- & $=$ & $=$ \\
\hline All stands & 18.4 & - & -- & 2.8 & 6.8 & 8.8 \\
\hline \multicolumn{7}{|l|}{ Maple-beech } \\
\hline Sawt imber & 692.3 & -- & 259.5 & 355.0 & 54.9 & 22.9 \\
\hline Poletimber & 110.5 & -- & 29.7 & 62.2 & 13.8 & 4.8 \\
\hline Sapling $\&$ seedling & 181.9 & $=$ & 43.1 & 89.4 & 28.9 & 20.5 \\
\hline All stands & 984.7 & $=$ & 332.3 & 506.6 & 97.6 & 48.2 \\
\hline \multicolumn{7}{|c|}{ Cherry-ash-yel low-poplar } \\
\hline Sawt imber & 394.7 & -- & 218.8 & 143.1 & 24.9 & 7.9 \\
\hline Poletimber & 112.3 & -- & 32.3 & 61.7 & 7.6 & 10.7 \\
\hline Sapling seedling & 142.0 & -- & 26.4 & 68.6 & 31.8 & 15.2 \\
\hline All stands & 649.0 & $\therefore$ & 277.5 & 273.4 & 64.3 & 33.8 \\
\hline Nonstucked & 40.5 & -- & $\cdots$ & 3.6 & 14.1 & 22.8 \\
\hline \multicolumn{7}{|l|}{ All types } \\
\hline Sawt imber & $2,770.0$ & -- & 520.2 & 978.0 & 932.0 & 339.8 \\
\hline Poletimber & 673.5 & $\cdots$ & 83.5 & 265.0 & 203.8 & 121.2 \\
\hline Sapling \& seedling & 811.8 & -- & 92.0 & 249.9 & 250.4 & $219 . b$ \\
\hline Nonstocked & 40.5 & $=$ & - & 3.6 & 14.1 & 22.8 \\
\hline All stands & $4,295.8$ & $=$ & 695.7 & $1,496.5$ & $1,400.3$ & 703.3 \\
\hline
\end{tabular}




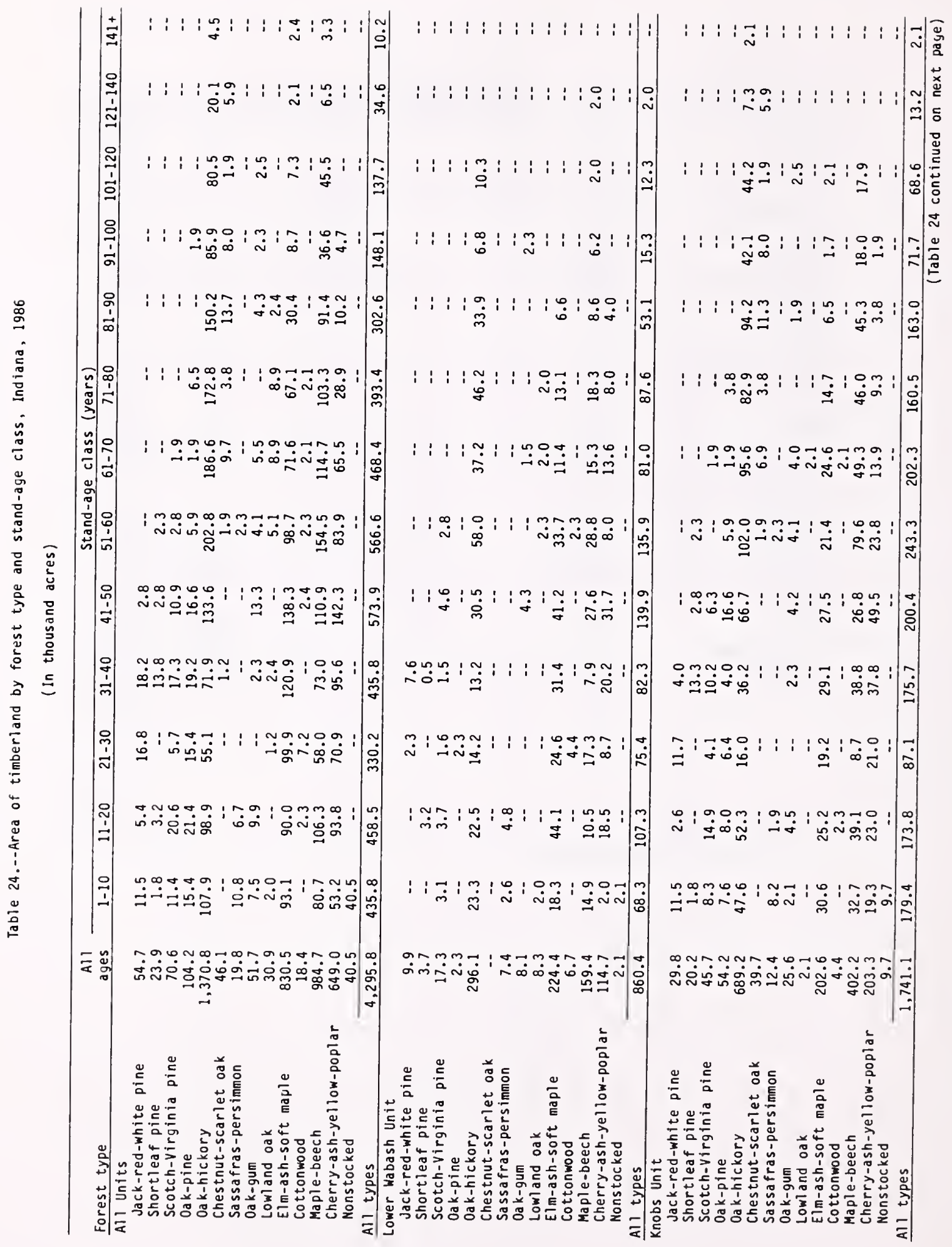




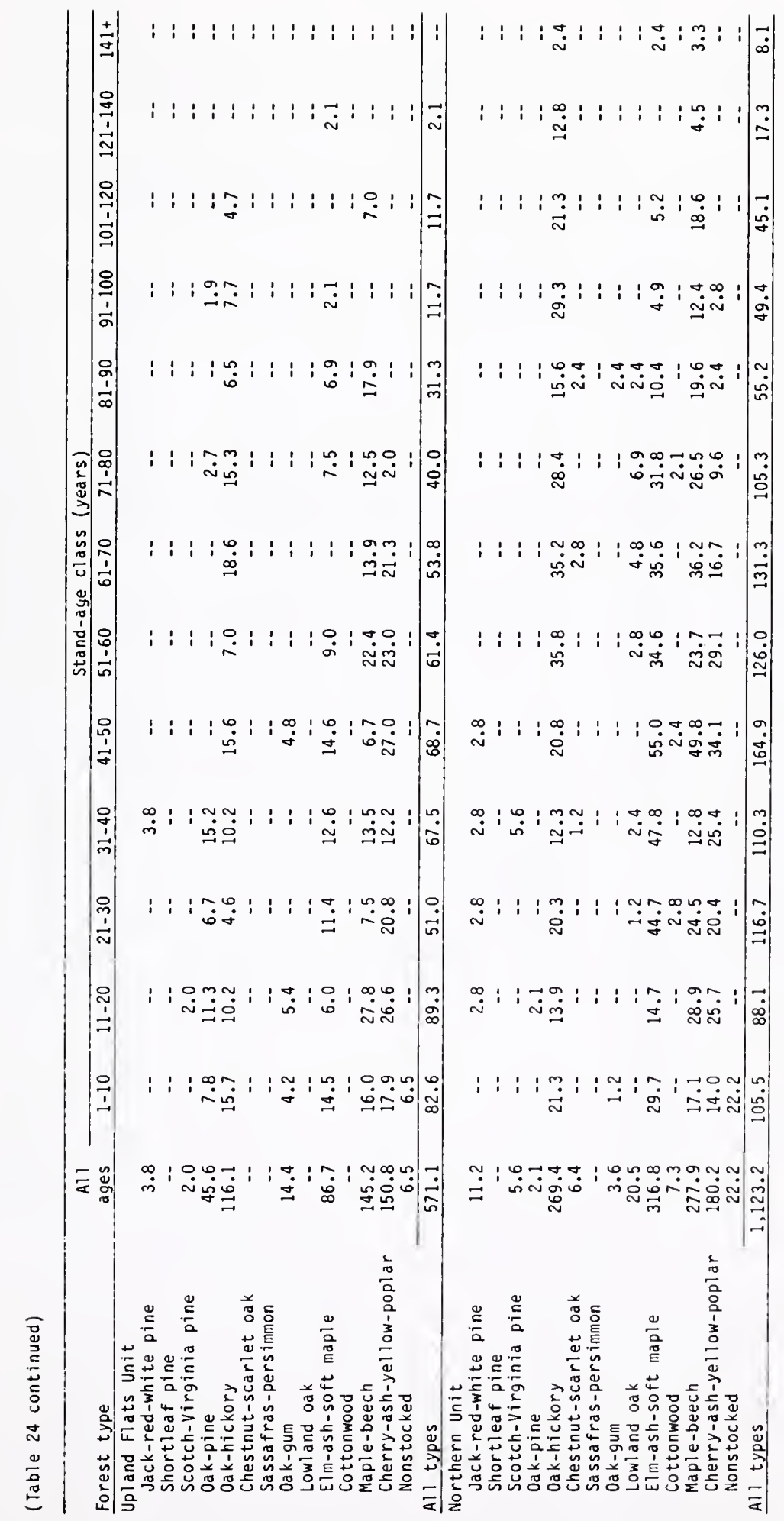


Table 25.--Area of timberland by forest type, site-index class, and Forest Survey Unit, Indiana, 1986

(In thousand acres)

\begin{tabular}{|c|c|c|c|c|c|c|c|c|c|c|}
\hline \multirow[b]{2}{*}{ Unit and forest type } & \multirow{2}{*}{$\begin{array}{c}\text { All } \\
\text { classes }\end{array}$} & \multicolumn{9}{|c|}{ Site-index class (feet) } \\
\hline & & $11-20$ & $21-30$ & $31-40$ & $41-50$ & $51-60$ & $61-70$ & $71-80$ & $81-90$ & $91+$ \\
\hline $\begin{array}{l}\text { All Units } \\
\text { Jack-red-white pine } \\
\text { Shortleaf pine } \\
\text { Scotch-Virginia pine } \\
\text { Oak-pine } \\
\text { Oak-hickory } \\
\text { Chestnut-scarlet oak } \\
\text { Sassafras-persimmon } \\
\text { Oak-gum } \\
\text { Lowland oak } \\
\text { Elm-ash-soft maple } \\
\text { Cottonwood } \\
\text { Maple-beech } \\
\text { Cherry-ash-yellow-poplar } \\
\text { Nonstocked }\end{array}$ & $\begin{array}{r}54.7 \\
23.9 \\
70.6 \\
104.2 \\
1,370.8 \\
46.1 \\
19.8 \\
51.7 \\
30.9 \\
830.5 \\
18.4 \\
984.7 \\
649.0 \\
40.5 \\
\end{array}$ & $\begin{array}{l}-- \\
-- \\
-- \\
-- \\
-- \\
-- \\
-- \\
-- \\
-- \\
-- \\
-- \\
-- \\
-- \\
--\end{array}$ & $\begin{array}{l}-- \\
-- \\
-- \\
-- \\
-- \\
-- \\
-- \\
-- \\
-- \\
-- \\
-- \\
-- \\
-- \\
--\end{array}$ & $\begin{array}{r}-. \\
-. \\
6.3 \\
2.6 \\
4.7 \\
-. \\
-. \\
-. \\
-.- \\
2.7 \\
-. \\
-- \\
-. \\
3.8\end{array}$ & $\begin{array}{r}-. \\
-- \\
-- \\
5.5 \\
34.8 \\
2.1 \\
-- \\
1.2 \\
-- \\
13.6 \\
-- \\
6.1 \\
2.8 \\
3.8 \\
\end{array}$ & $\begin{array}{r}-- \\
1.8 \\
3.6 \\
-- \\
125.0 \\
4.0 \\
-- \\
2.7 \\
6.9 \\
41.5 \\
4.2 \\
42.1 \\
31.0 \\
11.9\end{array}$ & $\begin{array}{r}-- \\
0.4 \\
15.6 \\
15.5 \\
296.2 \\
13.7 \\
4.7 \\
19.5 \\
-- \\
108.6 \\
4.6 \\
97.6 \\
64.3 \\
6.2 \\
\end{array}$ & $\begin{array}{r}5.1 \\
4.0 \\
14.4 \\
33.3 \\
366.8 \\
21.1 \\
6.2 \\
11.9 \\
6.5 \\
167.6 \\
-.- \\
162.7 \\
76.1 \\
6.7\end{array}$ & $\begin{array}{r}2.8 \\
14.1 \\
16.9 \\
17.9 \\
338.0 \\
5.2 \\
2.1 \\
6.3 \\
10.4 \\
191.5 \\
6.8 \\
254.2 \\
150.2 \\
5.7 \\
\end{array}$ & $\begin{array}{r}46.8 \\
3.6 \\
13.8 \\
29.4 \\
205.3 \\
-. \\
6.8 \\
10.1 \\
7.1 \\
305.0 \\
2.8 \\
422.0 \\
324.6 \\
2.4 \\
\end{array}$ \\
\hline All types & $4,295.8$ & -- & -- & 20.1 & 69.9 & 274.7 & 646.9 & 882.4 & $1,022.1$ & $1,379.7$ \\
\hline $\begin{array}{l}\text { Lower Wabash Unit } \\
\text { Jack-red-white pine } \\
\text { Shortleaf pine } \\
\text { Scotch-Virginia pine } \\
\text { 0ak-pine } \\
\text { Oak-hickory } \\
\text { Chestnut-scarlet oak } \\
\text { Sassafras-persimmon } \\
\text { 0ak-gum } \\
\text { Lowland oak } \\
\text { Elm-ash-soft maple } \\
\text { Cottonwood } \\
\text { Maple-beech } \\
\text { Cherry-ash-yellow-poplar } \\
\text { Nonstocked }\end{array}$ & $\begin{array}{r}9.9 \\
3.7 \\
17.3 \\
2.3 \\
296.1 \\
-- \\
7.4 \\
8.1 \\
8.3 \\
224.4 \\
6.7 \\
159.4 \\
114.7 \\
2.1 \\
\end{array}$ & $\begin{array}{l}-- \\
-- \\
-- \\
-- \\
-- \\
-- \\
-- \\
-- \\
-- \\
-- \\
-- \\
-- \\
--\end{array}$ & $\begin{array}{l}-- \\
-- \\
-- \\
-- \\
-- \\
-- \\
-- \\
-- \\
-- \\
-- \\
-- \\
-- \\
--\end{array}$ & $\begin{array}{c}-- \\
-- \\
-- \\
-- \\
2.3 \\
-- \\
-- \\
-- \\
-- \\
-- \\
-- \\
-- \\
-- \\
-- \\
\end{array}$ & $\begin{array}{l}-- \\
-- \\
-- \\
-- \\
-- \\
-- \\
-- \\
-- \\
-- \\
4.8 \\
-- \\
-- \\
-- \\
--\end{array}$ & $\begin{array}{r}-- \\
-- \\
1.6 \\
-- \\
16.2 \\
-- \\
-- \\
-- \\
-- \\
12.4 \\
2.1 \\
10.0 \\
4.4 \\
--\end{array}$ & $\begin{array}{r}-- \\
-- \\
5.2 \\
-- \\
54.2 \\
-- \\
2.6 \\
3.8 \\
-- \\
28.8 \\
2.3 \\
16.1 \\
9.6 \\
--\end{array}$ & $\begin{array}{r}2.3 \\
-- \\
4.4 \\
-. \\
85.2 \\
-- \\
2.2 \\
-- \\
2.0 \\
27.1 \\
-- \\
19.5 \\
7.8 \\
--\end{array}$ & $\begin{array}{r}-. \\
0.5 \\
2.3 \\
-. \\
89.1 \\
-. \\
-- \\
2.3 \\
4.0 \\
56.3 \\
2.3 \\
35.7 \\
23.2 \\
2.1 \\
\end{array}$ & $\begin{array}{r}7.6 \\
3.2 \\
3.8 \\
2.3 \\
49.1 \\
-. \\
2.6 \\
2.0 \\
2.3 \\
95.0 \\
- \\
78.1 \\
69.7 \\
.-\end{array}$ \\
\hline Al 1 types & 860.4 & - & -- & 2.3 & 4.8 & 46.7 & 122.6 & 150.5 & 217.8 & 315.7 \\
\hline $\begin{array}{l}\text { Knobs Unit } \\
\text { Jack-red-white pine } \\
\text { Shortleaf pine } \\
\text { Scotch-Virginia pine } \\
\text { Oak-pine } \\
\text { Oak-hickory } \\
\text { Chestnut-scarlet oak } \\
\text { Sassafras-persimmon } \\
\text { Oak-gum } \\
\text { Lowland oak } \\
\text { Elm-ash-soft maple } \\
\text { Cottonwood } \\
\text { Maple-beech } \\
\text { Cherry-ash-yellow-poplar } \\
\text { Nonstocked }\end{array}$ & $\begin{array}{r}29.8 \\
20.2 \\
45.7 \\
54.2 \\
689.2 \\
39.7 \\
12.4 \\
25.6 \\
2.1 \\
202.6 \\
4.4 \\
402.2 \\
203.3 \\
9.7 \\
\end{array}$ & $\begin{array}{l}-- \\
-- \\
-- \\
-- \\
-- \\
-- \\
-- \\
-- \\
-- \\
-- \\
-- \\
-- \\
-- \\
-- \\
\end{array}$ & $\begin{array}{l}-- \\
-- \\
-- \\
-- \\
-- \\
-- \\
-- \\
-- \\
-- \\
-- \\
-- \\
-- \\
-- \\
-\end{array}$ & $\begin{array}{c}-- \\
-- \\
4.3 \\
2.6 \\
-- \\
-- \\
-- \\
-- \\
-- \\
-- \\
-- \\
-- \\
-- \\
3.8 \\
\end{array}$ & $\begin{array}{r}-- \\
-- \\
-- \\
-- \\
22.1 \\
2.1 \\
-- \\
-- \\
-- \\
6.4 \\
-- \\
4.0 \\
-- \\
-- \\
\end{array}$ & $\begin{array}{r}-- \\
1.8 \\
2.0 \\
-- \\
73.9 \\
4.0 \\
-- \\
-- \\
2.1 \\
4.5 \\
-- \\
12.1 \\
4.2 \\
2.1 \\
\end{array}$ & $\begin{array}{r}-- \\
0.4 \\
10.4 \\
7.6 \\
157.1 \\
13.7 \\
2.1 \\
8.8 \\
-.- \\
15.6 \\
2.3 \\
30.9 \\
18.4 \\
3.8 \\
\end{array}$ & $\begin{array}{r}-- \\
4.0 \\
7.2 \\
24.0 \\
190.7 \\
19.9 \\
4.0 \\
6.8 \\
-- \\
40.5 \\
-- \\
74.6 \\
19.4 \\
--- \\
\end{array}$ & $\begin{array}{r}-- \\
13.6 \\
14.6 \\
8.3 \\
173.1 \\
-- \\
2.1 \\
1.9 \\
-.- \\
43.3 \\
2.1 \\
113.8 \\
36.5 \\
--\end{array}$ & $\begin{array}{r}29.8 \\
0.4 \\
7.2 \\
11.7 \\
72.3 \\
-. \\
4.2 \\
8.1 \\
-- \\
92.3 \\
-.- \\
166.8 \\
124.8 \\
---\end{array}$ \\
\hline All types. & $1,741.1$ & $\ldots$ & $\because$ & 10.7 & 34.6 & 106.7 & 271.1 & 391.1 & 409.3 & 517.6 \\
\hline
\end{tabular}

(Table 25 continued on next paye) 
(Table 25 continued)

\begin{tabular}{|c|c|c|c|c|c|c|c|c|c|c|}
\hline \multirow[b]{2}{*}{ Unit and forest type } & \multirow{2}{*}{$\begin{array}{c}\text { All } \\
\text { classes }\end{array}$} & \multicolumn{9}{|c|}{ Site-index class (feet) } \\
\hline & & $11-20$ & $21-30$ & $31-40$ & $41-50$ & $51-60$ & $61-70$ & $71-80$ & $81-90$ & $91+$ \\
\hline \multicolumn{11}{|l|}{ Upland Flats Unit } \\
\hline Shortleaf pine & -- & -- & -. & - & - & - & - & - & - & $\begin{array}{ll}0.0 \\
-1\end{array}$ \\
\hline Scotch-Virginia pine & 2.0 & -. & -. & 2.0 & -- & -- & -- & -- & -- & -- \\
\hline Oak-pine & 45.6 & -- & -- & - & 5.5 & -- & 7.9 & 9.3 & 9.6 & 13.3 \\
\hline Oak-hickory & 116.1 & -- & -. & -- & 0.5 & 12.2 & 20.7 & 25.5 & 21.1 & 36.1 \\
\hline Chestnut-scarlet oak & -- & -- & -- & -- & -- & - & -- & -- & -- & -- \\
\hline Sassafras-persimmon & -- & -- & -- & -- & -- & -- & -- & -- & -- & -- \\
\hline 0ak-gum & 14.4 & -- & -- & -- & -- & 2.7 & 6.9 & 2.7 & 2.1 & -- \\
\hline Lowl and oak & -- & -- & -. & -- & -- & 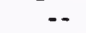 & -- & $\ldots$ & - & -. \\
\hline Elm-ash-soft maple & 86.7 & -- & -- & 2.7 & -- & 12.8 & 20.5 & 22.1 & 14.0 & 14.6 \\
\hline Cottonwood & -- & -- & -- & -- & -- & -- & - & -- & -- & -- \\
\hline Maple-beech & 145.2 & -- & -- & -- & 2.1 & 6.2 & 23.2 & 13.4 & 37.4 & 62.9 \\
\hline Cherry-ash-yellow-poplar & . 150.8 & -- & -- & -- & -- & 19.6 & 16.5 & 20.5 & 48.7 & 45.5 \\
\hline Nons tocked & 6.5 & -- & -- & -- & 3.8 & -- & -- & 2.7 & -- & -- \\
\hline All types & 571.1 & -- & -. & 4.7 & 11.9 & 53.5 & 95.7 & 96.2 & 132.9 & 176.2 \\
\hline \multicolumn{11}{|l|}{ Northern Unit } \\
\hline Jack-red-white pine & 11.2 & -- & -- & -- & -- & -- & -. & 2.8 & 2.8 & 5.6 \\
\hline Shortleaf pine & 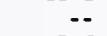 & -- & -- & -. & -- & -- & -- & -- & -- & -- \\
\hline Scotch-Virginia pine & 5.6 & -- & -- & -- & -- & -- & -- & 2.8 & -- & 2.8 \\
\hline oak-pine & 2.1 & -- & -- & -- & -- & -- & -- & -- & -- & 2.1 \\
\hline oak-hickory & 269.4 & -- & -. & 2.4 & 12.2 & 22.7 & 64.2 & 65.4 & 54.7 & 47.8 \\
\hline Chestnut-scarlet oak & 6.4 & -- & -- & -- & -- & -- & -- & 1.2 & 5.2 & -- \\
\hline Sassafras-persimmon & -- & -- & -- & -- & -- & -- & -- & -- & -- & -- \\
\hline 0ak-gum & 3.6 & -- & -- & -- & 1.2 & -- & -- & 2.4 & -- & -- \\
\hline Lowl and oak & 20.5 & -- & -. & -- & -- & 4.8 & -- & 4.5 & 6.4 & 4.8 \\
\hline Elm-ash-soft maple & 316.8 & -- & -- & -- & 2.4 & 11.8 & 43.7 & 77.9 & 77.9 & 103.1 \\
\hline Cottonwood & 7.3 & -- & -- & -- & -- & 2.1 & -- & -- & 2.4 & 2.8 \\
\hline Maple-beech & 277.9 & -- & -- & -- & -- & 13.8 & 27.4 & 55.2 & 67.3 & 114.2 \\
\hline Cherry-ash-yellow-poplar & - 180.2 & -- & -- & -- & 2.8 & 2.8 & 19.8 & 28.4 & 41.8 & 84.6 \\
\hline Nonstocked & 22.2 & -. & -- & -- & -- & 9.8 & 2.4 & 4.0 & 3.6 & 2.4 \\
\hline All types & $1,123.2$ & -. & -- & 2.4 & 18.6 & 67.8 & 157.5 & 244.6 & 262.1 & 370.2 \\
\hline
\end{tabular}




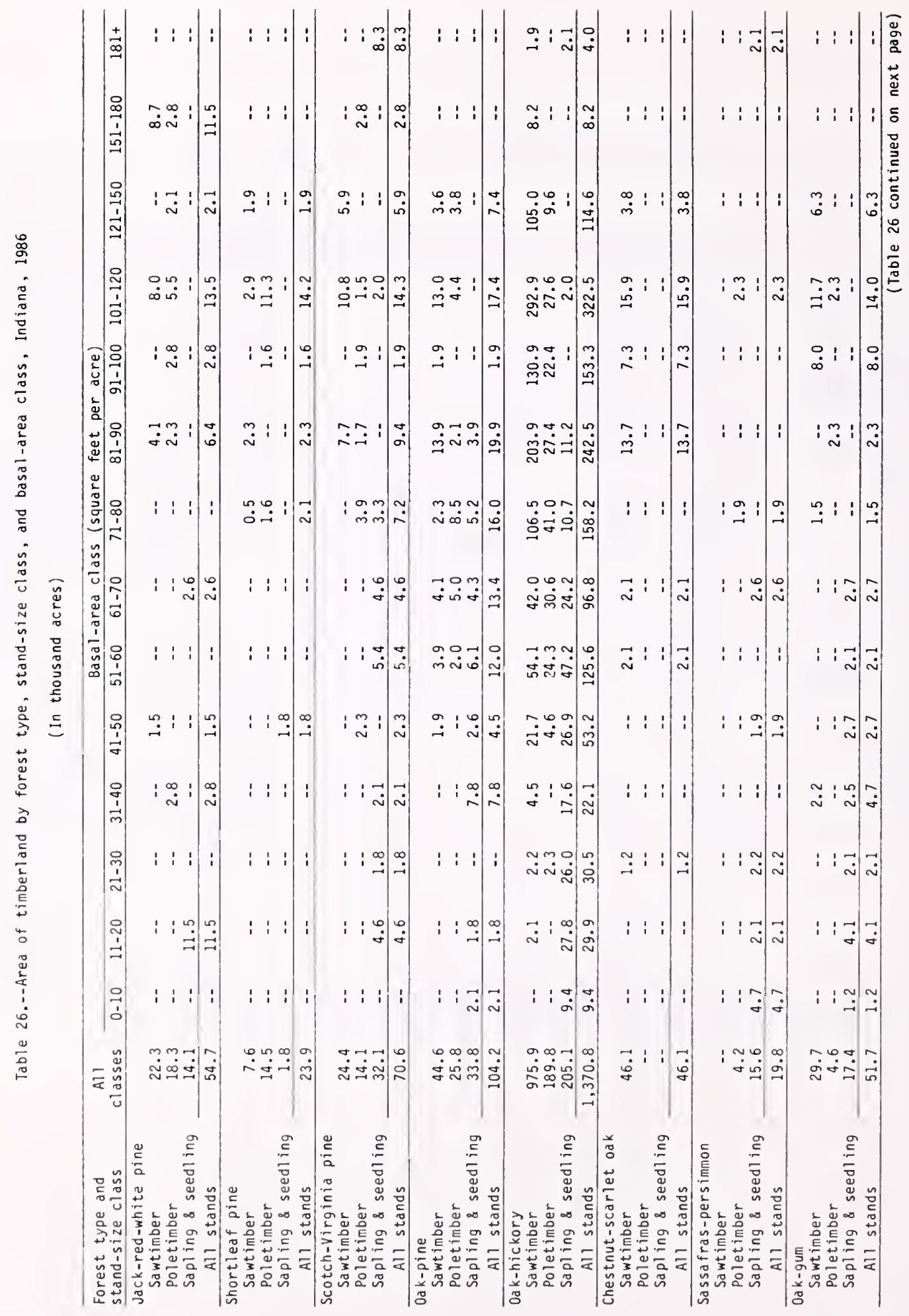




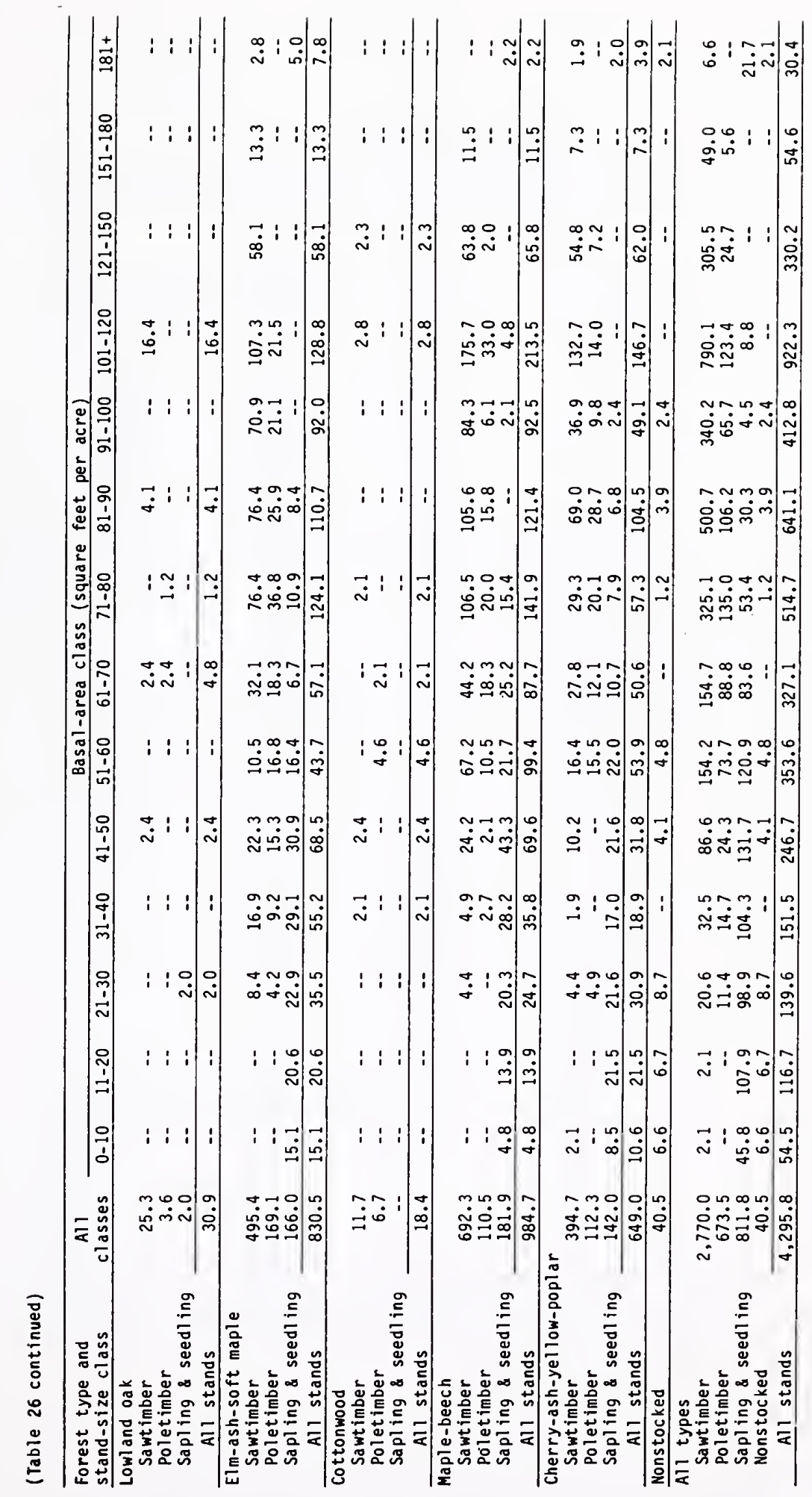


Table 27.--Area of timberland by stocking class based on selected stand components and Forest Survey Unit, lndiana, 1986

(In thousand acres)

\begin{tabular}{|c|c|c|c|}
\hline \multirow{2}{*}{$\begin{array}{l}\text { Stocking } \\
\text { class } \\
\text { (percent) }\end{array}$} & \multicolumn{3}{|c|}{ Stocking classified in terms of: } \\
\hline & $\begin{array}{c}\text { All } \\
\text { live trees }\end{array}$ & $\begin{array}{l}\text { Growing- } \\
\text { stock trees }\end{array}$ & $\begin{array}{l}\text { Rough and } \\
\text { rotten trees }\end{array}$ \\
\hline $\begin{array}{c}\text { A1 Units } \\
0-10 \\
11-20 \\
21-30 \\
31-40 \\
41-50 \\
51-60 \\
61-70 \\
71-80 \\
81-90 \\
91-100 \\
101-110 \\
111-120 \\
121-130 \\
131-140 \\
141-150 \\
151-160 \\
161+\end{array}$ & $\begin{array}{r}2.1 \\
12.5 \\
11.4 \\
21.6 \\
37.4 \\
78.1 \\
98.6 \\
168.5 \\
250.5 \\
417.8 \\
653.8 \\
884.9 \\
816.3 \\
536.7 \\
232.5 \\
70.6 \\
2.5\end{array}$ & $\begin{array}{r}18.3 \\
28.7 \\
52.7 \\
76.6 \\
157.7 \\
232.6 \\
321.2 \\
411.6 \\
543.2 \\
683.8 \\
622.2 \\
572.3 \\
338.5 \\
172.2 \\
57.7 \\
6.5 \\
-.\end{array}$ & $\begin{array}{r}1,379.0 \\
1,173.7 \\
759.2 \\
455.1 \\
299.4 \\
133.3 \\
49.6 \\
30.9 \\
5.6 \\
5.5 \\
2.1 \\
2.4 \\
-- \\
-- \\
-- \\
-. \\
-.\end{array}$ \\
\hline All classes & $4,295.8$ & $4,295.8$ & $4,295.8$ \\
\hline $\begin{array}{c}\text { Lower Wabash } \\
0-10 \\
11-20 \\
21-30 \\
31-40 \\
41-50 \\
51-60 \\
61-70 \\
71-80 \\
81-90 \\
91-100 \\
101-110 \\
111-120 \\
121-130 \\
131-140 \\
141-150 \\
151-160 \\
161+\end{array}$ & $\begin{array}{r}-.- \\
2.1 \\
-- \\
5.2 \\
4.3 \\
11.0 \\
16.5 \\
48.8 \\
32.9 \\
86.8 \\
156.7 \\
199.1 \\
171.2 \\
71.3 \\
50.5 \\
4.0 \\
-.-\end{array}$ & $\begin{array}{r}2.1 \\
.- \\
7.1 \\
6.2 \\
21.8 \\
29.7 \\
69.6 \\
74.1 \\
127.1 \\
162.1 \\
130.1 \\
108.4 \\
81.4 \\
31.2 \\
9.5 \\
. .- \\
. .\end{array}$ & $\begin{array}{r}325.3 \\
257.2 \\
151.1 \\
71.6 \\
38.8 \\
8.8 \\
7.6 \\
-. \\
-. \\
-- \\
-. \\
-. \\
-- \\
-- \\
-. \\
-- \\
-.\end{array}$ \\
\hline All classes & 860.4 & 860.4 & 860.4 \\
\hline
\end{tabular}


(Table 27 continued)

\begin{tabular}{|c|c|c|c|}
\hline \multirow{2}{*}{$\begin{array}{l}\text { Stocking } \\
\text { class } \\
\text { (percent) }\end{array}$} & \multicolumn{3}{|c|}{ Stocking classified in terms of: } \\
\hline & $\begin{array}{l}\text { All } \\
\text { live trees }\end{array}$ & $\begin{array}{c}\text { Growing- } \\
\text { stock trees }\end{array}$ & $\begin{array}{l}\text { Rough and } \\
\text { rotten trees }\end{array}$ \\
\hline $\begin{array}{c}\text { Knobs Unit } \\
0-10 \\
11-20 \\
21-30 \\
31-40 \\
41-50 \\
51-60 \\
61-70 \\
71-80 \\
81-90 \\
91-100 \\
101-110 \\
111-120 \\
121-130 \\
131-140 \\
141-150 \\
151-160 \\
161+\end{array}$ & $\begin{array}{r}2.1 \\
3.8 \\
4.3 \\
1.8 \\
21.3 \\
21.9 \\
24.6 \\
51.7 \\
108.5 \\
158.6 \\
269.6 \\
375.6 \\
356.7 \\
242.9 \\
69.8 \\
27.8 \\
0.1 \\
\end{array}$ & $\begin{array}{r}5.9 \\
3.9 \\
9.0 \\
16.5 \\
35.6 \\
47.5 \\
72.7 \\
147.2 \\
200.2 \\
283.9 \\
290.5 \\
319.1 \\
182.0 \\
95.1 \\
25.5 \\
6.5 \\
--- \\
\end{array}$ & $\begin{array}{r}767.9 \\
562.2 \\
248.9 \\
99.6 \\
46.1 \\
10.5 \\
5.9 \\
-.- \\
-- \\
-- \\
-. \\
-- \\
-- \\
-- \\
-- \\
-. \\
-- \\
\end{array}$ \\
\hline All classes & $1,741.1$ & $1,741.1$ & $1,741.1$ \\
\hline $\begin{array}{l}\text { Upland Flats Unit } \\
0-10 \\
11-20 \\
21-30 \\
31-40 \\
41-50 \\
51-60 \\
61-70 \\
71-80 \\
81-90 \\
91-100 \\
101-110 \\
111-120 \\
121-130 \\
131-140 \\
141-150 \\
151-160 \\
161+\end{array}$ & $\begin{array}{r}-- \\
2.1 \\
3.8 \\
6.2 \\
5.7 \\
5.7 \\
14.8 \\
18.8 \\
52.1 \\
39.7 \\
83.1 \\
98.1 \\
115.2 \\
73.5 \\
38.8 \\
13.5 \\
-.- \\
\end{array}$ & $\begin{array}{r}1.7 \\
8.9 \\
10.5 \\
19.1 \\
16.7 \\
67.2 \\
59.4 \\
57.0 \\
80.3 \\
67.4 \\
67.1 \\
56.1 \\
30.0 \\
22.3 \\
7.4 \\
-. \\
-. \\
\end{array}$ & $\begin{array}{r}102.1 \\
111.3 \\
144.3 \\
99.0 \\
49.9 \\
34.7 \\
10.9 \\
9.7 \\
4.4 \\
2.7 \\
2.1 \\
-. \\
-. \\
-- \\
-. \\
-- \\
-. \\
\end{array}$ \\
\hline All classes & 571.1 & 571.1 & 571.1 \\
\hline $\begin{array}{c}\text { Northern Unit } \\
0-10 \\
11-20 \\
21-30 \\
31-40 \\
41-50 \\
51-60 \\
61-70 \\
71-80 \\
81-90 \\
91-100 \\
101-110 \\
111-120 \\
121-130 \\
131-140 \\
141-150 \\
151-160 \\
161+\end{array}$ & $\begin{array}{r}-- \\
4.5 \\
3.3 \\
8.4 \\
6.1 \\
39.5 \\
42.7 \\
49.2 \\
57.0 \\
132.7 \\
144.4 \\
212.1 \\
173.2 \\
149.0 \\
73.4 \\
25.3 \\
2.4 \\
\end{array}$ & $\begin{array}{r}8.6 \\
15.9 \\
26.1 \\
34.8 \\
83.6 \\
88.2 \\
119.5 \\
133.3 \\
135.6 \\
170.4 \\
134.5 \\
88.7 \\
45.1 \\
23.6 \\
15.3 \\
-- \\
--. \\
\end{array}$ & $\begin{array}{r}183.7 \\
243.0 \\
214.9 \\
184.9 \\
164.6 \\
79.3 \\
25.2 \\
21.2 \\
1.2 \\
2.8 \\
-- \\
2.4 \\
-- \\
-- \\
-- \\
-- \\
--\end{array}$ \\
\hline All classes & $1,123.2$ & $1,123.2$ & $1,123.2$ \\
\hline
\end{tabular}


Table 28.--Area of timberland in plantations by forest type and stand-aye class, and Forest Survey Unit, 1986

(In thousand acres)

\begin{tabular}{|c|c|c|c|c|c|c|c|c|}
\hline \multirow[b]{2}{*}{ Unit and forest type } & \multirow{2}{*}{$\begin{array}{r}\text { Al1 } \\
\text { ages }\end{array}$} & \multicolumn{7}{|c|}{ Stand-age class (years) } \\
\hline & & $1-10$ & $11-20$ & $21-30$ & $31-40$ & $41-50$ & $50-60$ & $61-70$ \\
\hline \multicolumn{9}{|l|}{ All Units } \\
\hline Jack-red-white pine & 40.2 & 11.5 & 5.4 & 9.8 & 13.5 & $\because$ & -- & -. \\
\hline Shortleaf pine & 20.7 & 1.8 & 3.2 & -- & 13.4 & 0.4 & 1.9 & -. \\
\hline Scotch-Virginia pine & 34.2 & 3.1 & 7.8 & 2.3 & 12.8 & 6.3 & -. & 1.9 \\
\hline Oak-gum & 1.5 & -- & -- & -- & -- & -- & -- & 1.5 \\
\hline Lowl and oak & 1.2 & -- & - & 1.2 & $\because$ & - & -- & -- \\
\hline Elm-ash-soft-maple & 3.7 & -- & -- & 3.7 & -- & -- & -- & -- \\
\hline Cherry-ash-yellow-poplar & 3.7 & -- & 2.1 & 1.6 & -- & -- & -- & -- \\
\hline A11 types & 105.2 & 16.4 & 18.5 & 18.6 & 39.7 & 6.7 & 1.9 & 3.4 \\
\hline \multicolumn{9}{|l|}{ Lower Wabash Unit } \\
\hline Jack-red-white pine & 9.9 & -- & -. & 2.3 & 7.6 & -- & -- & -- \\
\hline Shortleaf pine & 3.7 & -- & 3.2 & -- & 0.5 & -- & -- & .. \\
\hline Scotch-Virginia pine & 6.7 & 3.1 & 2.1 & -- & 1.5 & -. & -. & -- \\
\hline Oak-gum & 1.5 & -- & -- & -- & -- & -- & -- & 1.5 \\
\hline Elm-ash-soft maple & 3.7 & -- & -- & 3.7 & -- & -- & -. & -- \\
\hline Cherry-ash-yellow-poplar & 1.6 & -. & $\because$ & 1.6 & -- & -- &.- & -- \\
\hline All types & 27.1 & 3.1 & 5.3 & 7.6 & 9.6 & $\cdots$ & $\because$ & 1.5 \\
\hline \multicolumn{9}{|l|}{ Knobs Unit } \\
\hline Jack-red-white pine & 23.7 & 11.5 & 2.6 & 7.5 & 2.1 & -- & थ & -- \\
\hline Shortleaf pine & 17.0 & 1.8 &.- & -- & 12.9 & 0.4 & 1.9 & -- \\
\hline Scotch-Virginia pine & 21.9 & -- & 5.7 & 2.3 & 5.7 & 6.3 & -- & 1.9 \\
\hline All types & 62.6 & 13.3 & 8.3 & 9.8 & 20.7 & 6.7 & 1.9 & 1.9 \\
\hline \multicolumn{9}{|l|}{ Upland Flats Unit } \\
\hline Jack-red-white pine & 3.8 & -- & -- & .. & 3.8 & -- & -- & -- \\
\hline Cherry-ash-yellow-poplar & 2.1 & -- & 2.1 & $\because$ & - & -- & $\because$ & -- \\
\hline All types & 5.9 & -- & 2.1 & $\ldots$ & 3.8 & -- & $\ldots$ & $\ldots$ \\
\hline \multicolumn{9}{|l|}{ Northern Unit } \\
\hline Jack-red-white pine & 2.8 & -- & 2.8 & .- & -. & -- & $\therefore$ & -- \\
\hline Scotch-Virginia pine & 5.6 & -- & -- & -- & 5.6 & -- & -- & - \\
\hline Lowl and oak & 1.2 & -- & $\approx$ & 1.2 & -- & -- & -- & $-=$ \\
\hline All types & 9.6 & $\because$ & 2.8 & 1.2 & 5.6 & - & -- & $\cdots$ \\
\hline
\end{tabular}


Table 29.--Area of reserved timberland by ownership class and Forest Survey Unit, Indiana, 1986

(In thousand acres)

\begin{tabular}{lrrrrr} 
& & \multicolumn{3}{c}{ Forest Survey Unit } \\
\cline { 3 - 6 } & All & $\begin{array}{c}\text { Lower } \\
\text { Wabash } \\
\text { Onnership class }\end{array}$ & $\begin{array}{c}\text { Knobs } \\
\text { Unit }\end{array}$ & $\begin{array}{c}\text { Upland } \\
\text { Flats } \\
\text { Unit }\end{array}$ & $\begin{array}{c}\text { Northern } \\
\text { Unit }\end{array}$ \\
\hline National forest & 12.4 & -- & 12.4 & -- & -- \\
Miscellaneous federal & 16.7 & -- & -- & 15.9 & 0.8 \\
State & 92.3 & 14.5 & 47.4 & 14.4 & 16.0 \\
County and municipal & 11.4 & -- & 2.1 & -- & 9.3 \\
Forest industry & -- & -- & -- & -- & -- \\
Farmer & -- & -- & -- & -- & - \\
Misc. private-corp. & 9.1 & 2.0 & 2.3 & 2.8 & 2.0 \\
Misc. private-indiv. & 1.5 & 0.7 & -- & -- & 0.8 \\
All owners & 143.4 & 17.2 & 64.2 & 33.1 & 28.9 \\
\hline
\end{tabular}

Table 30.--Area of reserved timberland by forest type and Forest Survey Unit, Indiana, 1986

(In thousand acres)

\begin{tabular}{|c|c|c|c|c|c|}
\hline \multirow[b]{2}{*}{ Ownership class } & \multirow[b]{2}{*}{$\begin{array}{c}\text { All } \\
\text { Units }\end{array}$} & \multicolumn{4}{|c|}{ Forest Survey Unit } \\
\hline & & $\begin{array}{c}\text { Lower } \\
\text { Wabash } \\
\text { Unit }\end{array}$ & $\begin{array}{l}\text { Knobs } \\
\text { Unit }\end{array}$ & $\begin{array}{l}\text { Upland } \\
\text { Flats } \\
\text { Unit }\end{array}$ & $\begin{array}{c}\text { Northern } \\
\text { Unit }\end{array}$ \\
\hline Jack-red-white pine & 6.8 & 2.1 & -- & 4.7 & - \\
\hline Shortleaf pine & 1.6 & -- & -- & 1.6 & -- \\
\hline Scotch-Virginia pine & -- & -- & -- & -- & -- \\
\hline Oak-pine & - & -- & -. & -- & -- \\
\hline 0ak-hickory & 79.8 & 12.3 & 45.5 & 10.0 & 12.0 \\
\hline Chestnut-scarlet oak & -- & - & -- & - & -- \\
\hline Sassafras-persimmon & 4.4 & -. & 4.4 & - & -- \\
\hline 0ak-gum & -- & -- & -- & -- & -- \\
\hline Lowl and oak & -- & -- & -- & -- & -- \\
\hline Elm-ash-soft maple & 9.3 & 2.8 & 4.1 & 1.6 & 0.8 \\
\hline Cottonwood & 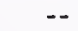 & -- & -- & -- & -- \\
\hline Maple-beech & 39.4 & -- & 10.2 & 15.2 & 14.0 \\
\hline Cherry-ash-yellow-poplar & 2.1 & -- & -- & -- & 2.1 \\
\hline Nons toc ked & -- & -- & -- & -- & -- \\
\hline All types & 143.4 & 17.2 & 64.2 & 33.1 & 28.9 \\
\hline
\end{tabular}




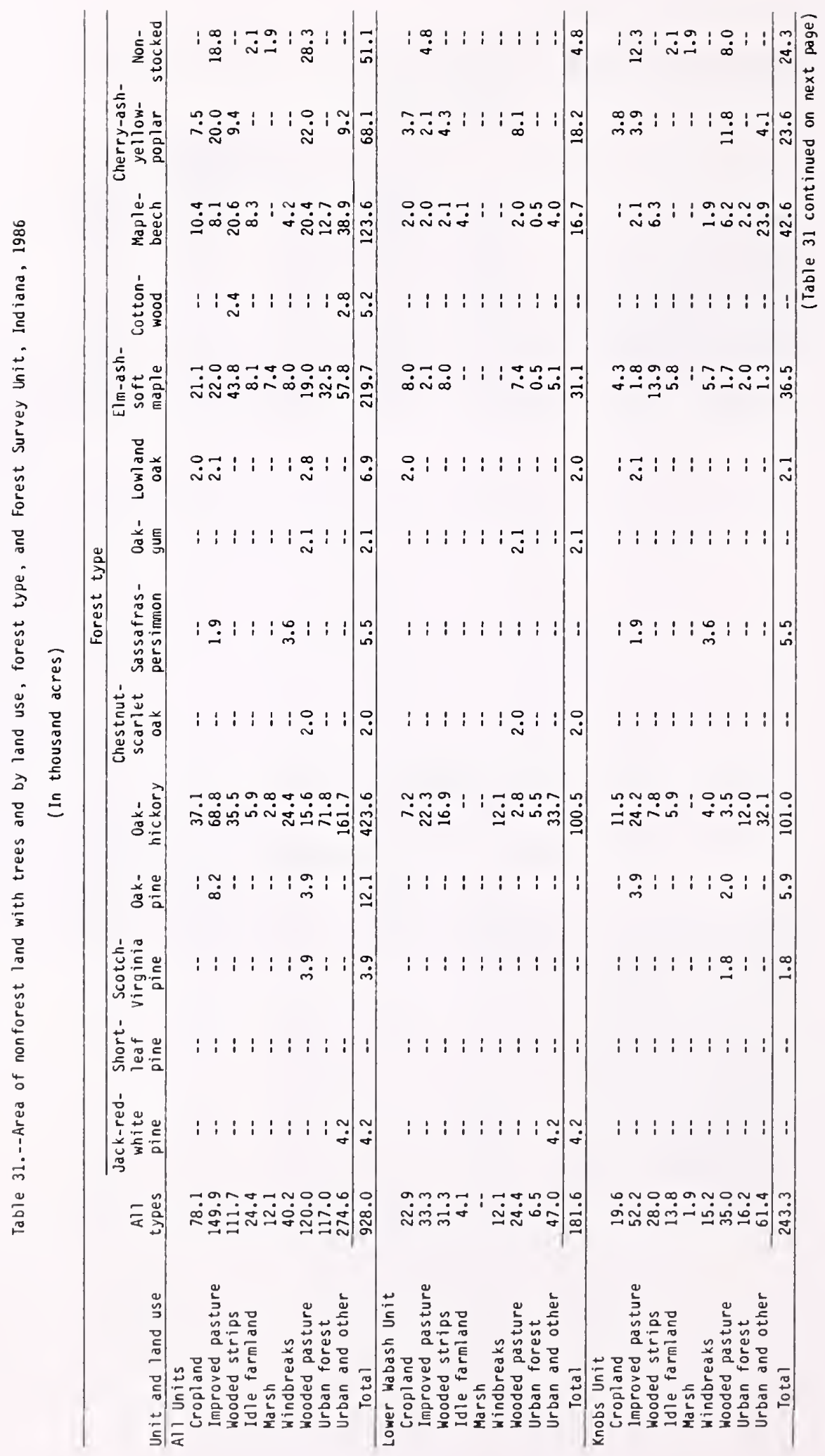




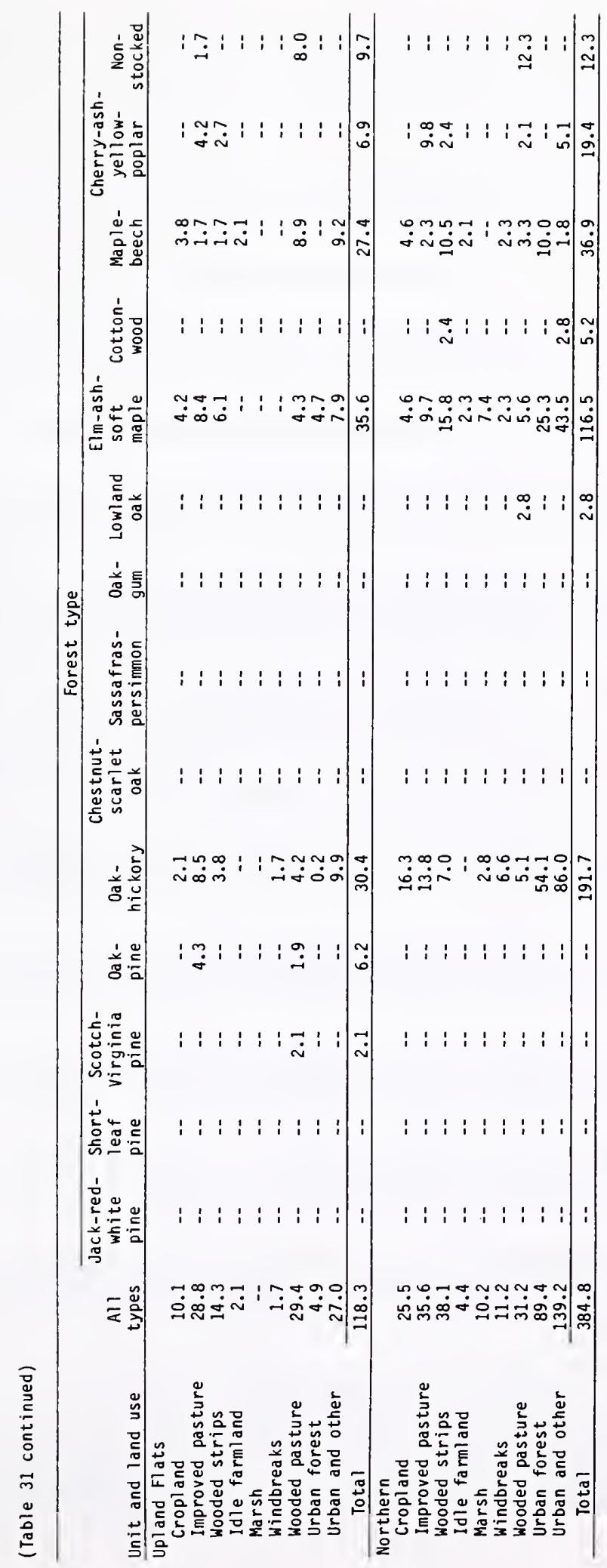




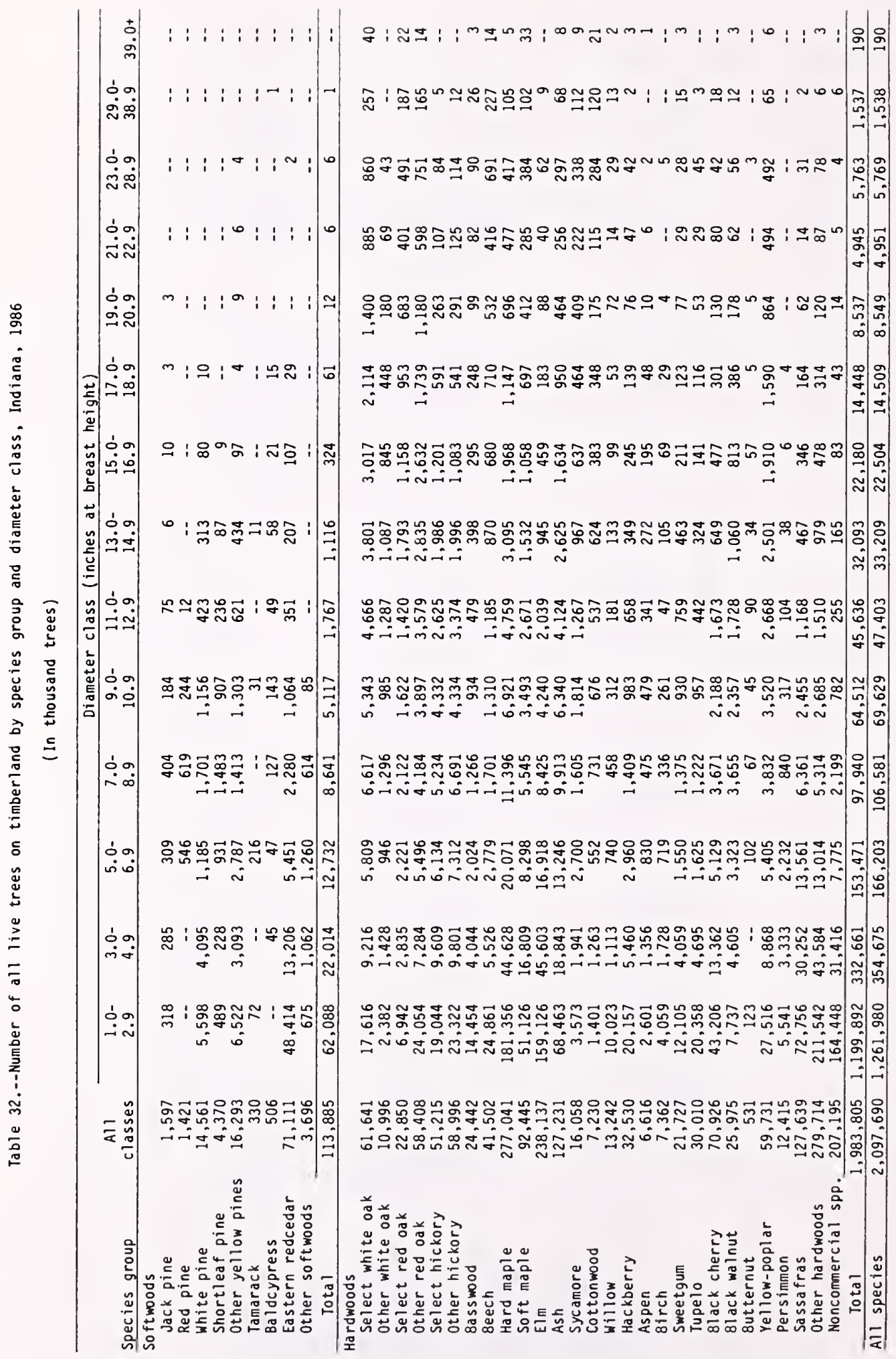




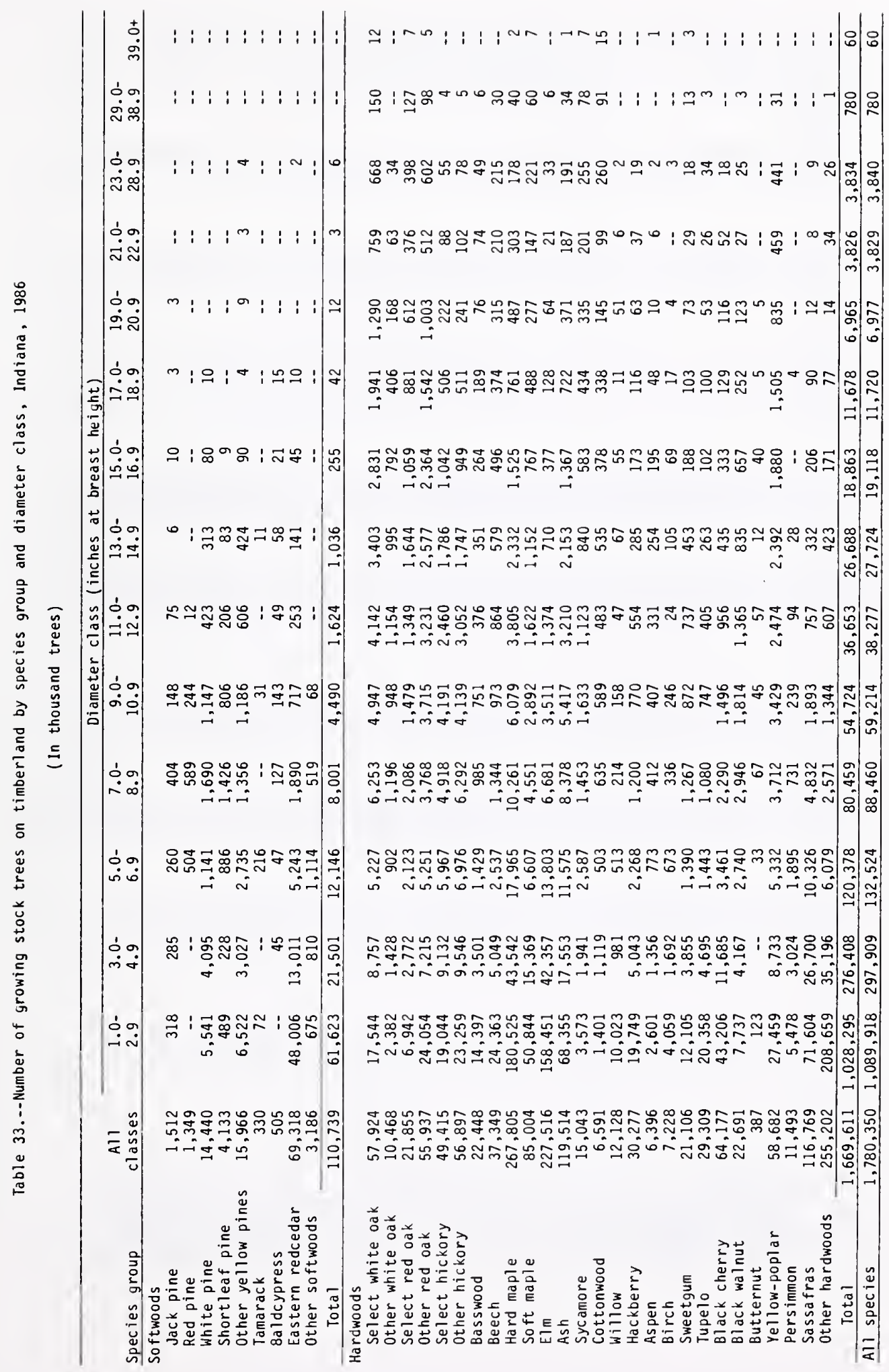




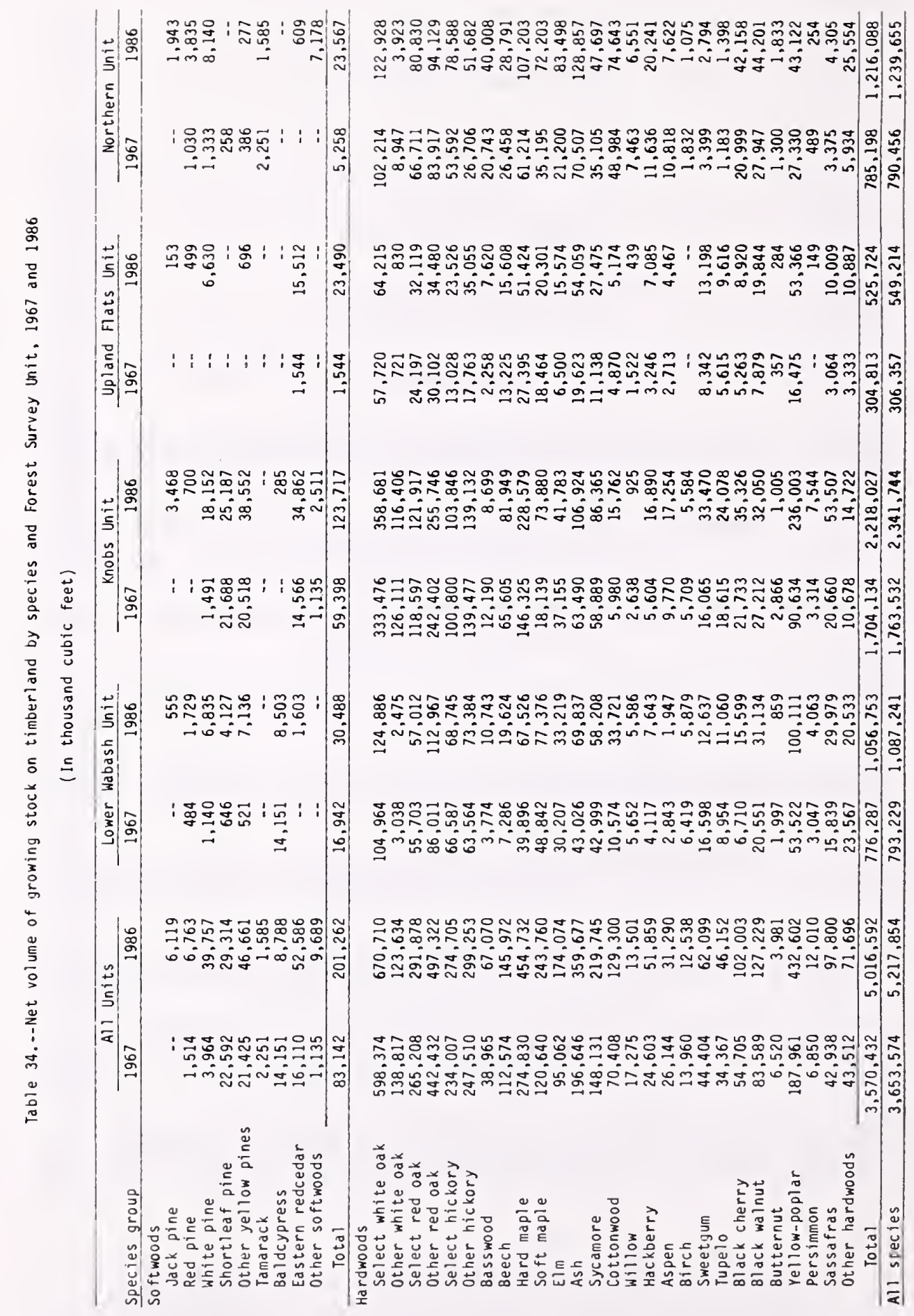




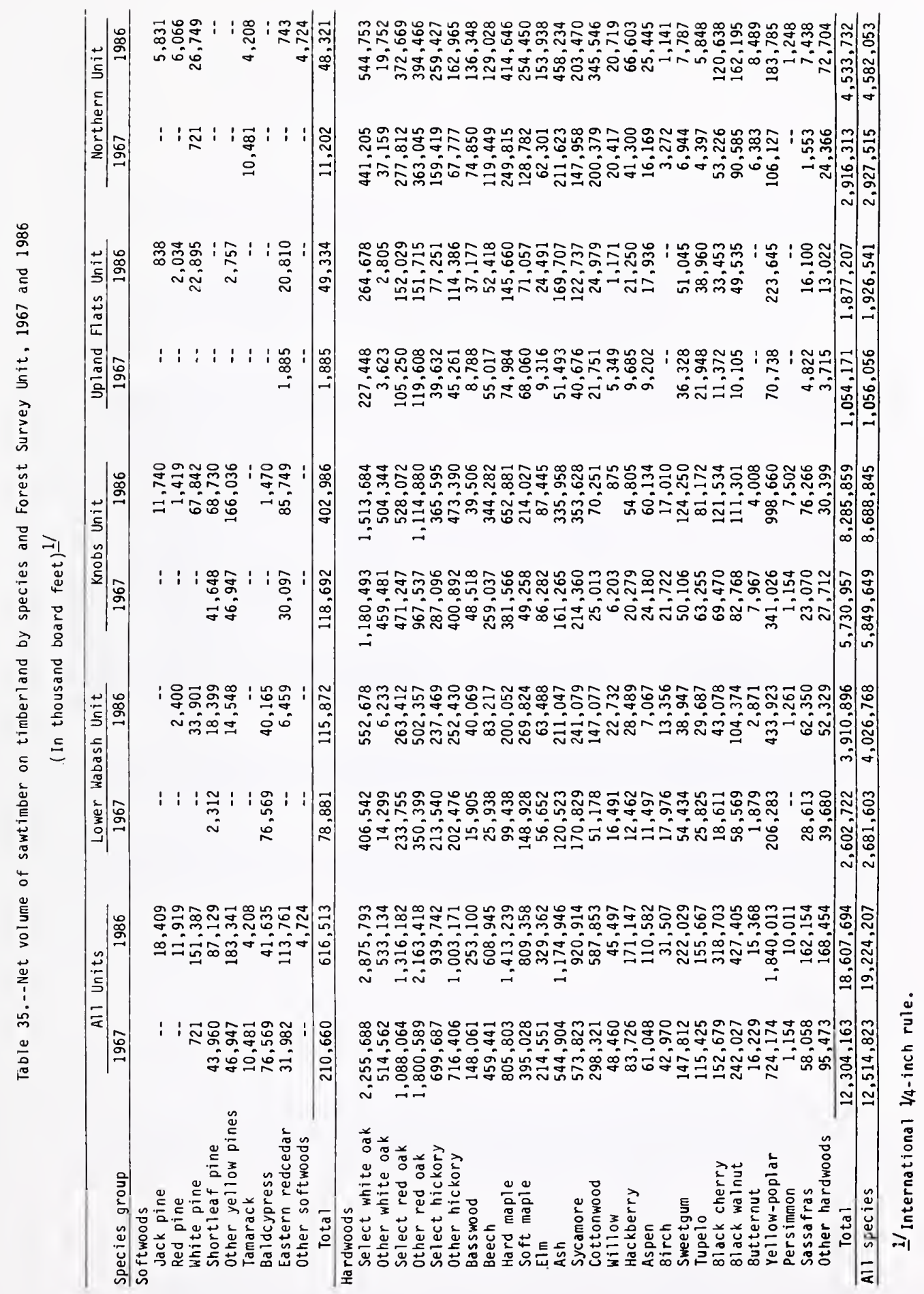




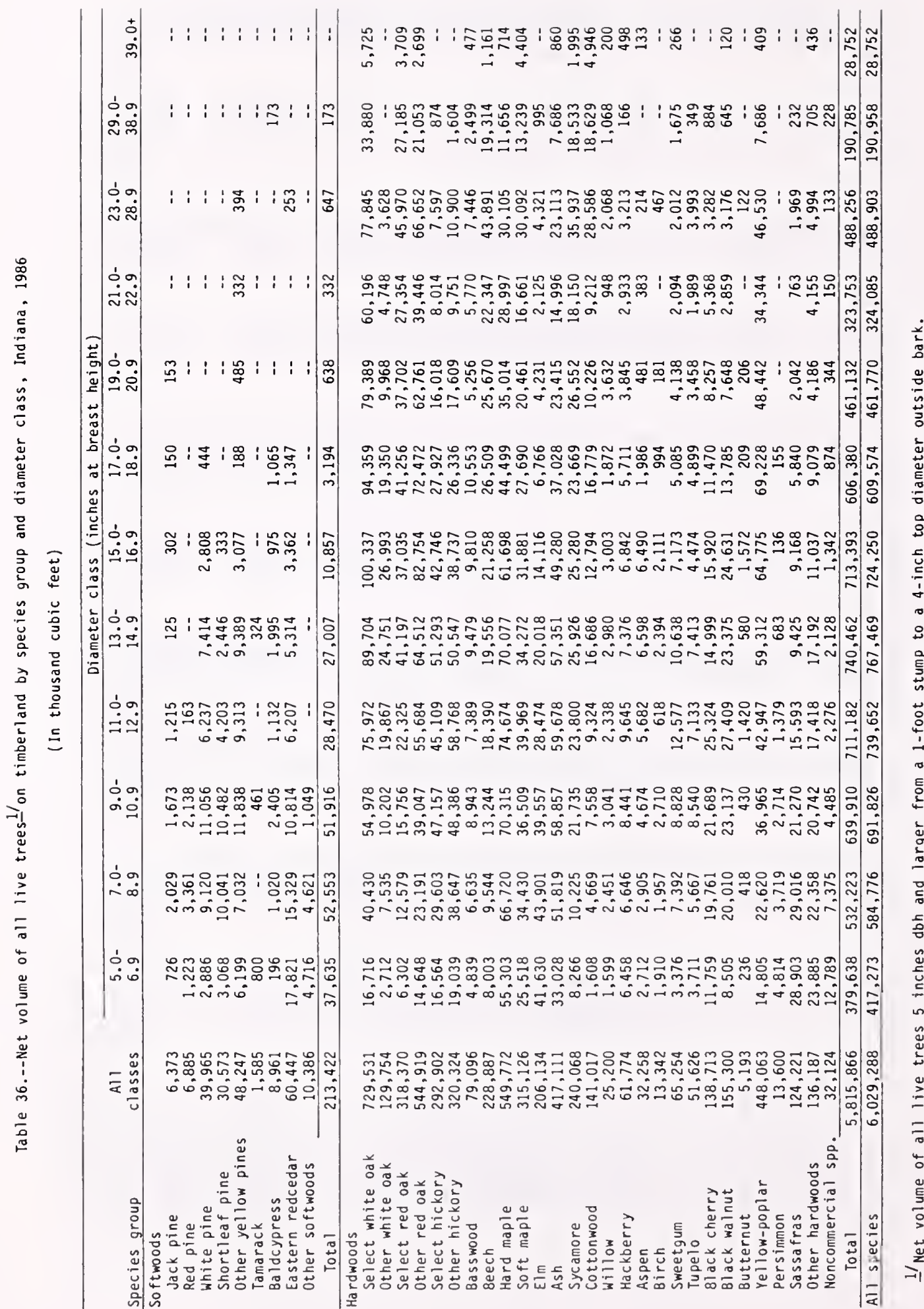


Table 37.--Net volume of timber on timberland by class of timber and species group, Indiana, 1986

(In thousand cubic feet)

\begin{tabular}{|c|c|c|c|c|c|}
\hline \multirow[b]{2}{*}{ Class of timber } & \multirow[b]{2}{*}{$\begin{array}{c}\text { All } \\
\text { species }\end{array}$} & \multicolumn{4}{|c|}{ Species group } \\
\hline & & Pine & $\begin{array}{c}\text { Other } \\
\text { softwoods }\end{array}$ & $\begin{array}{c}\text { Soft } \\
\text { hardwoods }\end{array}$ & $\begin{array}{c}\text { Hard } \\
\text { hardwoods }\end{array}$ \\
\hline $\begin{array}{l}\text { Live trees } \\
\text { Growing-stock trees } \\
\text { Sawtimber } \\
\text { Saw log portion } \\
\text { Upper stem portion }\end{array}$ & $\begin{array}{r}2,941,160 \\
823,450 \\
\end{array}$ & $\begin{array}{r}77,953 \\
6,666 \\
\end{array}$ & $\begin{array}{r}27,123 \\
2,259 \\
\end{array}$ & $\begin{array}{l}914,181 \\
264,619 \\
\end{array}$ & $\begin{array}{r}1,921,903 \\
549,906 \\
\end{array}$ \\
\hline Total & $3,764,610$ & 84,619 & 29,382 & $1,178,800$ & $2,471,809$ \\
\hline Poletimber & $1,453,244$ & 53,684 & 33,577 & 519,204 & 846,779 \\
\hline All growing-stock trees & $5,217,854$ & 138,303 & 62,959 & $1,698,004$ & $3,318,588$ \\
\hline $\begin{array}{l}\text { Cull trees } \\
\text { Short-log trees } \\
\text { Rough trees }\end{array}$ & 162,268 & 923 & 885 & 51,457 & 109,003 \\
\hline $\begin{array}{l}\text { Sawt imber } \\
\text { Poletimber }\end{array}$ & $\begin{array}{l}305,817 \\
168,004 \\
\end{array}$ & $\begin{array}{l}1,865 \\
1,288\end{array}$ & $\begin{array}{l}5,560 \\
1,546 \\
\end{array}$ & $\begin{array}{r}101,413 \\
76,133 \\
\end{array}$ & $\begin{array}{r}196,979 \\
89,037 \\
\end{array}$ \\
\hline Total & 473,821 & 3,153 & 7,106 & 177,546 & 286,016 \\
\hline $\begin{array}{l}\text { Rotten trees } \\
\text { Sawtimber } \\
\text { Poletimber }\end{array}$ & $\begin{array}{r}154,634 \\
20,711 \\
\end{array}$ & 50 & 43 & $\begin{array}{l}49,909 \\
10,779 \\
\end{array}$ & $\begin{array}{r}104,725 \\
9,839 \\
\end{array}$ \\
\hline Total & 175,345 & 50 & 43 & 60,688 & 114,564 \\
\hline A11 cull trees & 811,434 & 4,126 & 8,034 & 289,691 & 509,583 \\
\hline All live trees & $6,029,288$ & 142,429 & 70,993 & $1,987,695$ & $3,828,171$ \\
\hline $\begin{array}{l}\text { Salvable dead trees } \\
\text { Sawtimber } \\
\text { Poletimber }\end{array}$ & $\begin{array}{l}52,654 \\
29,343 \\
\end{array}$ & $\begin{array}{l}1,652 \\
1,533\end{array}$ & $\begin{array}{r}852 \\
1,299 \\
\end{array}$ & $\begin{array}{l}11,100 \\
13,688\end{array}$ & $\begin{array}{l}15,739 \\
36,134\end{array}$ \\
\hline Tota 1 & 81,997 & 3,185 & 2,151 & 24,788 & 51,873 \\
\hline All classes & $6,111,285$ & 145,614 & 73,144 & $2,012,483$ & $3,880,044$ \\
\hline
\end{tabular}




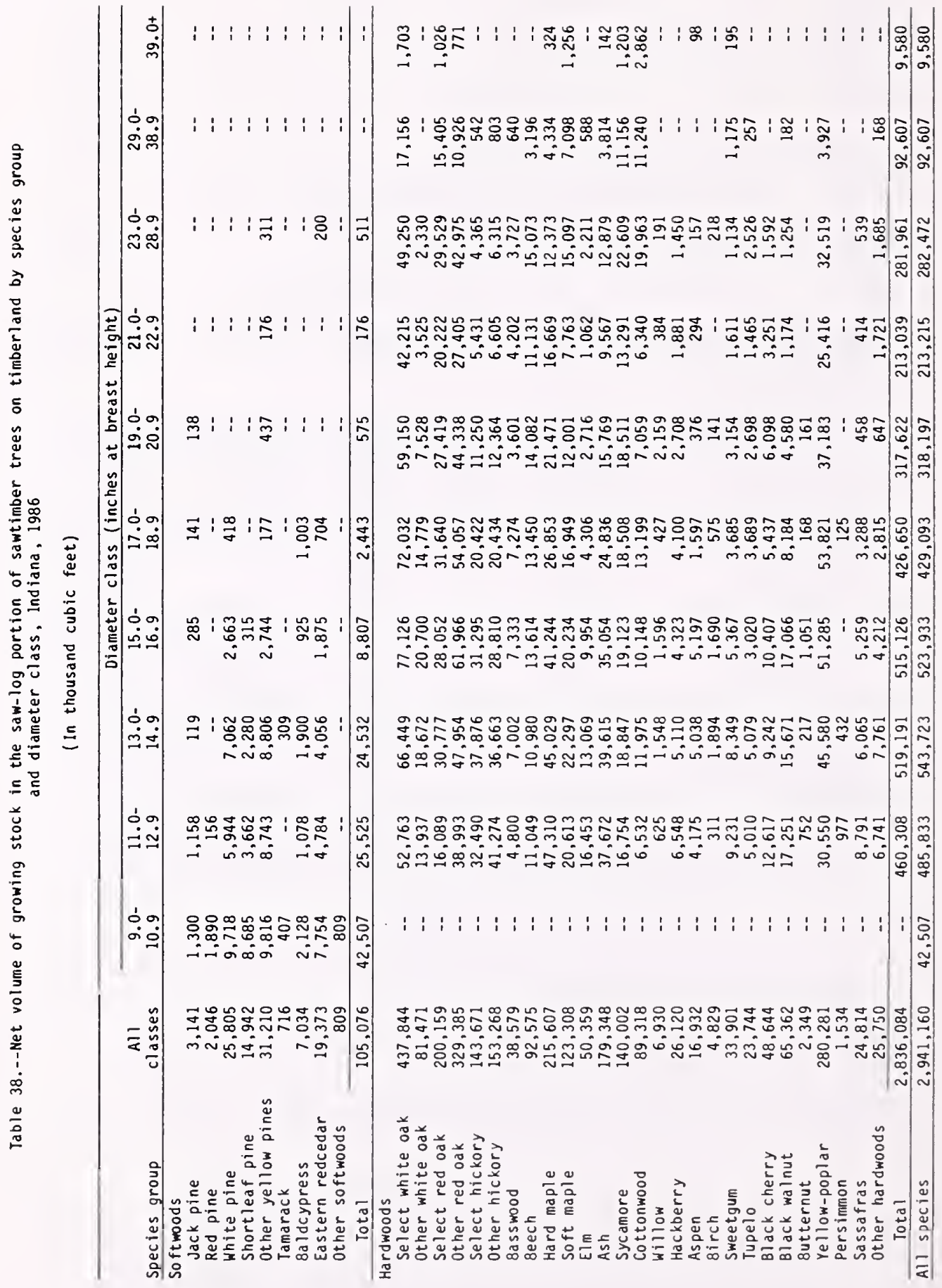




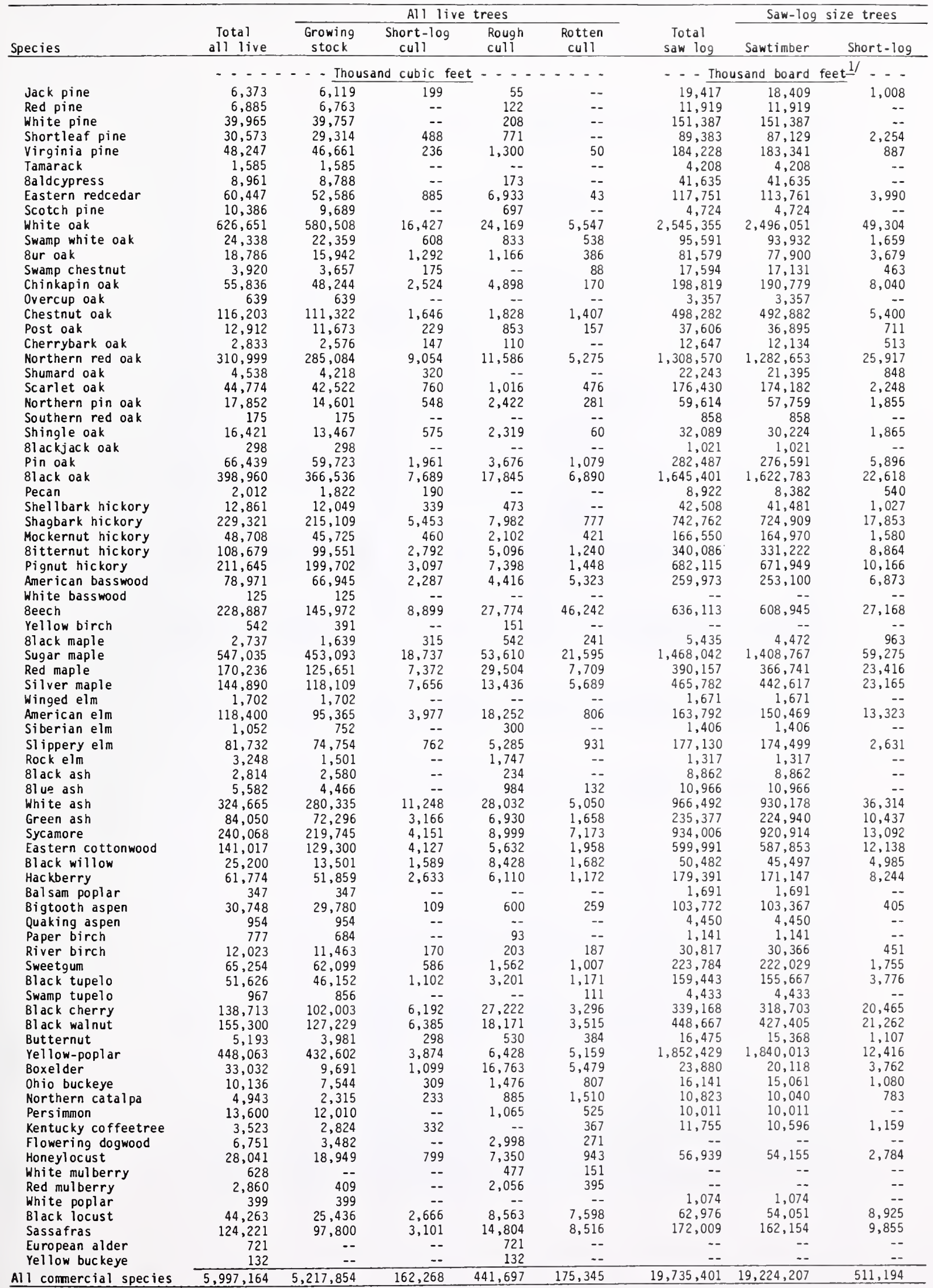

1/International $1 / 4$-inch rule. 


\begin{tabular}{|c|c|}
\hline Species & Cull volume \\
\hline $\begin{array}{l}\text { Osage-orange } \\
\text { Eastern ho phornbeam } \\
\text { Eastern redbud } \\
\text { Hawthorn } \\
\text { American hornbeam } \\
\text { Apple } \\
\text { Ailanthus } \\
\text { Wild plum } \\
\text { Pawpaw } \\
\text { Chokecherry }\end{array}$ & $\begin{array}{r}13,358 \\
5,973 \\
5,565 \\
3,349 \\
1,435 \\
1,385 \\
641 \\
196 \\
135 \\
87\end{array}$ \\
\hline All species & 32,124 \\
\hline
\end{tabular}




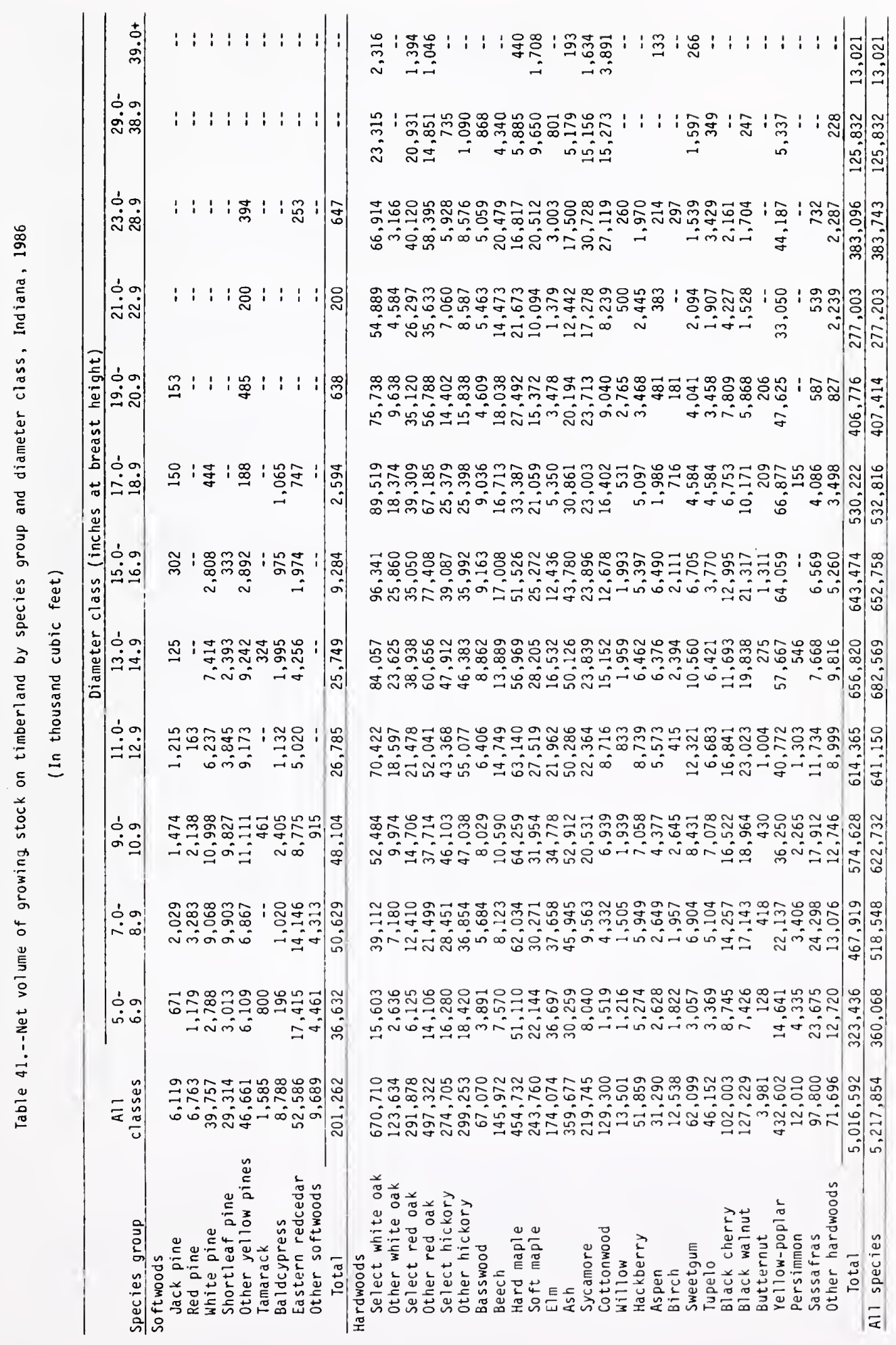




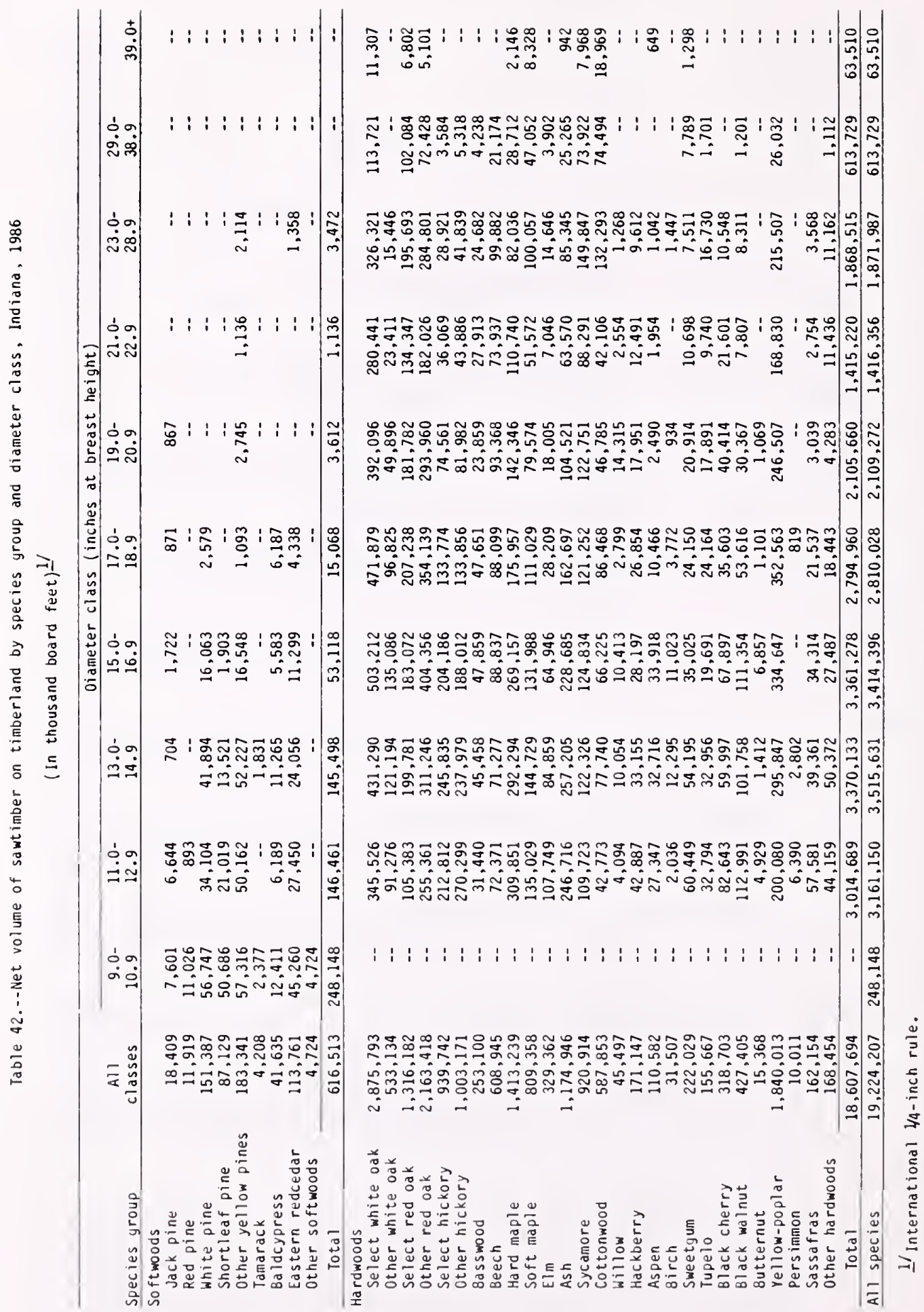




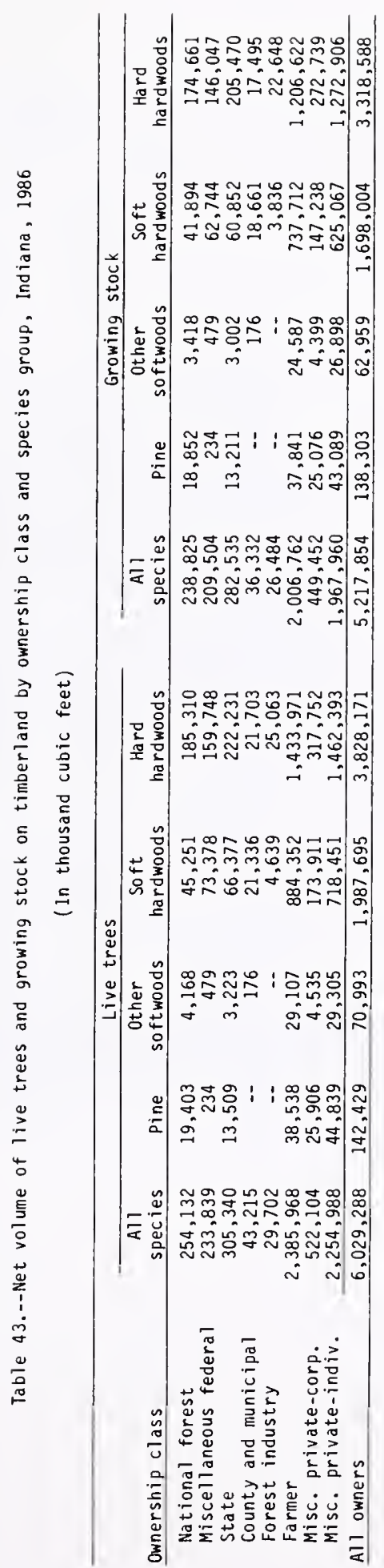


Table 44.--Net volume of growing stock on timberland by species group and forest type, lndiana, 1986

(In thousand cubic feet)

\begin{tabular}{|c|c|c|c|c|c|c|c|c|}
\hline \multirow[b]{2}{*}{ Species group } & \multirow[b]{2}{*}{$\begin{array}{l}\text { All } \\
\text { types }\end{array}$} & \multicolumn{7}{|c|}{ Forest type } \\
\hline & & $\begin{array}{l}\text { Jack-red- } \\
\text { white } \\
\text { pine }\end{array}$ & $\begin{array}{l}\text { Short- } \\
\text { leaf } \\
\text { pine }\end{array}$ & $\begin{array}{l}\text { Scotch- } \\
\text { Virginia } \\
\text { pine }\end{array}$ & $\begin{array}{l}\text { Oak - } \\
\text { pine }\end{array}$ & $\begin{array}{c}\text { Oak- } \\
\text { hickory }\end{array}$ & $\begin{array}{c}\text { Chestnut- } \\
\text { scarlet } \\
\text { oak }\end{array}$ & $\begin{array}{l}\text { Sassafras- } \\
\text { Persimnon }\end{array}$ \\
\hline \multicolumn{9}{|l|}{ So ftwoods } \\
\hline Jack pine & 6,119 & 4,221 & -- & - & 104 & 373 & -. & -- \\
\hline Red pine & 6,763 & 5,325 & -- & -- & $\cdots$ & 1,101 & - & -- \\
\hline White pine & 39,757 & 31,951 & -- & 948 & 2,753 & 2,016 & -- & -- \\
\hline Shortleaf pine & 29,314 & 4,508 & 19,327 & 1,431 & 794 & 2,180 & -- & $\cdots$ \\
\hline other yellow pines & 46,661 & 1,590 & 1,060 & 24,404 & 10,500 & 2,824 & -- & 460 \\
\hline Tamarack & 1,585 & 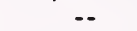 & - & - & -. & -- & -- & - \\
\hline Baldcypress & 8,788 & -- & - & - & -- & -- & - & -- \\
\hline Eastern redcedar & 52,586 & -- & 226 & 3,914 & 26,827 & 9,077 & - & - \\
\hline other softwoods & 9,689 & -- & $\ldots$ & 8,411 & - & - & -- & -- \\
\hline Total & 201,262 & 47,595 & 20,613 & 39,108 & 40,978 & 17,571 & -- & 460 \\
\hline \multicolumn{9}{|l|}{ Hardwoods } \\
\hline Select white oak & 670,710 & 711 & -- & 1,555 & 3,796 & 488,141 & 10,835 & 461 \\
\hline other white oak & 123,634 & -- & 146 & 1, & 1,884 & 59,958 & 49,860 & 137 \\
\hline Select red oak & 291,878 & 125 & 1,074 & 190 & 2,552 & 169,881 & 1,457 & -- \\
\hline other red oak & 497,322 & 168 & $\begin{array}{r}1,074 \\
278\end{array}$ & 3,249 & 8,946 & 345,175 & 12,574 & 401 \\
\hline Select hickory & 274,705 & 756 & 1,377 & 219 & 3,340 & 170,262 & 1,200 & -- \\
\hline other hickory & 299,253 & 688 & 157 & - & 4,438 & 175,275 & 2,833 & -- \\
\hline Basswood & 67,070 & -- & -- & -- & 2,136 & 9,386 & 238 & -- \\
\hline $8 \mathrm{eech}$ & 145,972 & -- & 51 & - & 2,120 & 21,737 & -. & -. \\
\hline Hard maple & 454,732 & 166 & 1,031 & 355 & 2,474 & 79,205 & 2,358 & 755 \\
\hline Soft maple & 243,760 & 719 & 239 & 624 & 763 & 17,475 & 460 & $\ldots$ \\
\hline Elm & 174,074 & 772 & 26 & 130 & 1,224 & 27,233 & - & -- \\
\hline Ash & 359,677 & 2,211 & 1,350 & 885 & 5,261 & 63,773 & -- & -- \\
\hline Sycamore & 219,745 & 1,822 & 365 & 3,051 & 1,049 & 23,083 & -- & -- \\
\hline Cottonwood & 129,300 & 1,908 & -- & 8,962 & - & 3,297 & -. & -. \\
\hline willow & 13,501 & -- & - & -- & - & 228 & -- & -- \\
\hline Hackberry & 51,859 & - & -- & -. & 97 & 4,177 & -. & -- \\
\hline Aspen & 31,290 & -- & - & - & -- & 13,723 & -- & -. \\
\hline Birch & 12,538 & - & - & 600 & -- & 789 & - & $\cdots$ \\
\hline Sweetgum & 62,099 & 549 & - & -- & 783 & 7,150 & $=$ & $\ldots$ \\
\hline Tupelo & 46,152 & 115 & 84 & 397 & 2,394 & 14,101 & 333 & 499 \\
\hline Black cherry & 102,003 & 1,239 & 763 & 1,092 & 1,155 & 17,965 & 1,388 & -- \\
\hline Black walnut & 127,229 & 10 & -. & 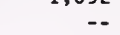 & 1,628 & 24,033 & -. & -- \\
\hline Butternut & 3,981 & -- & -- & -- & - & 128 & -- & -. \\
\hline Yellow-poplar & 432,602 & 1,725 & 4,152 & 1,747 & 6,534 & 86,684 & - & 1,131 \\
\hline Persimmon & 12,010 & -. & - & 523 & 288 & 4,181 & -- & 186 \\
\hline Sassafras & 97,800 & 117 & 1,751 & 564 & 993 & 26,098 & - & 2,472 \\
\hline other hardwoods & 71,696 & - & - & 422 & 345 & 10,756 & - & - \\
\hline Total & $5,016,592$ & 13,791 & 12,844 & 24,565 & 52,080 & $1,863,894$ & 83,536 & 6,042 \\
\hline All species & $5,217,854$ & 61,386 & 33,457 & 63,673 & 93,058 & $1,881,465$ & 83,536 & 6,502 \\
\hline
\end{tabular}


(Table 44 continued)

\begin{tabular}{|c|c|c|c|c|c|c|c|}
\hline \multirow[b]{2}{*}{ Species group } & \multicolumn{7}{|c|}{ Forest type } \\
\hline & $\begin{array}{l}\text { 0ak - } \\
\text { gum }\end{array}$ & $\begin{array}{c}\text { Lowl and } \\
\text { oak }\end{array}$ & $\begin{array}{c}\text { Elm-ash- } \\
\text { soft } \\
\text { maple }\end{array}$ & $\begin{array}{l}\text { Cotton- } \\
\text { wood }\end{array}$ & $\begin{array}{l}\text { Maple- } \\
\text { beech }\end{array}$ & $\begin{array}{c}\text { Cherry-ash- } \\
\text { yellow- } \\
\text { poplar }\end{array}$ & $\begin{array}{c}\text { Non- } \\
\text { stocked }\end{array}$ \\
\hline \multicolumn{8}{|l|}{ So ftwoods } \\
\hline Jack pine & -- & - & 153 & 101 & -- & 1,167 & -- \\
\hline Red pine & 232 & -- & 105 & -- & -- & -- & -- \\
\hline White pine & -- & -- & 1,040 & -- & 9 & 1,040 & -- \\
\hline Shortleaf pine & -- & -- & 517 & -- & -- & 557 & -- \\
\hline other yellow pines & 270 & -- & 1,702 & 494 & 2,934 & 423 & -- \\
\hline Tamarack & -- & -- & 1,585 & -- & -- & -- & -- \\
\hline 8aldcypress & 1,060 & -- & 7,728 & -- & -- & -- & -- \\
\hline Eastern redcedar & 151 & -- & 2,600 & -- & 4,273 & 5,518 & -- \\
\hline Other softwoods & 292 & -- & -- & -- & 160 & 826 & -- \\
\hline Total & 2,005 & - & 15,430 & 595 & 7,376 & 9,531 & -- \\
\hline \multicolumn{8}{|l|}{ Hardwoods } \\
\hline Select white oak & 3,002 & 7,645 & 33,106 & -- & 81,173 & 40,074 & 211 \\
\hline other white oak & 391 & 746 & 198 & -- & 8,196 & 2,118 & -- \\
\hline Select red oak & 1,403 & 300 & 11,222 & -- & 71,057 & 32,617 & -- \\
\hline other red oak & 7,046 & 18,447 & 35,574 & 147 & 38,267 & 26,574 & 476 \\
\hline Select hickory & 2,649 & 1,226 & 21,965 & -- & 53,441 & 18,270 & -- \\
\hline other hickory & - & 924 & 13,488 & -- & 64,675 & 36,775 & -- \\
\hline 8asswood & -- & 322 & 7,141 & -- & 40,188 & 7,659 & -- \\
\hline Beech & 302 & -- & 2,657 & -- & 111,691 & 9,534 & -- \\
\hline Hard maple & 422 & -- & 20,354 & -- & 304,307 & 43,305 & -- \\
\hline Soft maple & 7,534 & 1,172 & 155,719 & 3,841 & 40,249 & 14,965 & -- \\
\hline Elm & 652 & 1,814 & 58,985 & -- & 50,933 & 32,041 & 264 \\
\hline Ash & 3,238 & 2,938 & 94,275 & -- & 65,980 & 119,421 & 345 \\
\hline Sycamore & 3,651 & 86 & 105,534 & 1,128 & 38,448 & 40,205 & 1,323 \\
\hline Cottonwood & -- & 605 & 86,021 & 21,287 & 2,713 & 3,823 & 684 \\
\hline Willow & -- & -- & 11,883 & 697 & 313 & 277 & 103 \\
\hline Hackberry & -- & - & 28,614 & - & 11,338 & 7,633 & -- \\
\hline Aspen & 578 & -- & 2,352 & -- & 5,007 & 9,630 & -- \\
\hline 8irch & 918 & -- & 9,827 & - & -- & 404 & -- \\
\hline Sweetgum & 20,301 & 1,455 & 17,668 & - & 7,496 & 6,697 & -- \\
\hline Tupelo & 3,558 & 1,091 & 5,312 & -- & 12,714 & 5,554 & -- \\
\hline 81 ack cherry & 481 & 425 & 13,405 & -- & 37,170 & 26,780 & 140 \\
\hline 81 ack walnut & 396 & - & 29,651 & -- & 27,690 & 43,831 & -- \\
\hline 8utternut & -- & -- & 585 & -- & 3,062 & 206 & -- \\
\hline Yellow-poplar & 4,567 & - & 37,499 & 110 & 96,201 & 192,252 & -- \\
\hline Persimmon & 526 & -- & 1,339 & - & 4,143 & 824 & -- \\
\hline Sassafras & 1,949 & 556 & 15,116 & -- & 28,600 & 19,584 & -- \\
\hline Other hardwoods & 249 & 199 & 28,107 & 338 & 23,880 & 7,330 & 70 \\
\hline Total & 63,813 & 39,951 & 847,597 & 27,548 & $1,228,932$ & 748,383 & 3,616 \\
\hline All species & 65,818 & 39,951 & 863,027 & 28,143 & $1,236,308$ & 757,914 & 3,616 \\
\hline
\end{tabular}


Table 45.--Net volume of sawtimber on timberland by species group and forest type, Indiana, 1986 ( In thousand board feet) 1 /

\begin{tabular}{|c|c|c|c|c|c|c|c|c|}
\hline \multirow[b]{2}{*}{ Species group } & \multirow[b]{2}{*}{$\begin{array}{l}\text { All } \\
\text { types }\end{array}$} & \multicolumn{7}{|c|}{ Forest type } \\
\hline & & $\begin{array}{c}\text { Jack-red- } \\
\text { white } \\
\text { pine }\end{array}$ & $\begin{array}{l}\text { Short- } \\
\text { leaf } \\
\text { pine }\end{array}$ & $\begin{array}{l}\text { Scotch- } \\
\text { virginia } \\
\text { pine }\end{array}$ & $\begin{array}{l}\text { Oak- } \\
\text { pine }\end{array}$ & $\begin{array}{l}\text { Oak- } \\
\text { hickory }\end{array}$ & $\begin{array}{c}\text { Chestnut- } \\
\text { scarlet } \\
\text { oak }\end{array}$ & $\begin{array}{l}\text { Sassafras - } \\
\text { Persimmon }\end{array}$ \\
\hline \multicolumn{9}{|l|}{ Softwoods } \\
\hline Jack pine & 18,409 & 10,073 & -- & -- & 539 & 1,921 & - & -- \\
\hline Red pine & 11,919 & 8,466 & -- & -- & - & 3,453 & -- & -- \\
\hline White pine & 151,387 & 118,692 & -- & 579 & 13,678 & 10,728 & -- & -. \\
\hline Shortleaf pine & 87,129 & 16,448 & 50,918 & 5,677 & 4,093 & 4,875 & - & -- \\
\hline other yellow pines & 183,341 & 2,974 & 5,710 & 87,993 & 51,885 & 11,725 & - & 2,622 \\
\hline Tamarack & 4,208 & -- & - & -- & -- & -- & - & - \\
\hline Baldcypress & 41,635 & -- & -- & -- & -- & -- & -- & -- \\
\hline Eastern redcedar & 113,761 & -- & - & 9,073 & 61,713 & 21,582 & -- & -- \\
\hline other softwoods & 4,724 & -- & -- & 4,724 & -- & -- & - & -- \\
\hline Total & 616,513 & 156,653 & 56,628 & 108,046 & 131,908 & 54,284 & -- & 2,622 \\
\hline \multicolumn{9}{|l|}{ Hardwoods } \\
\hline Select white oak & $2,875,793$ & 3,490 & -- & 7,974 & 16,370 & $2,084,914$ & 45,833 & 1,594 \\
\hline other white oak & 533,134 & 0 & 751 & 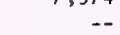 & 4,390 & 245,587 & 227,598 & 1,0 \\
\hline Select red oak & $1,316,182$ & -- & 5,658 & 971 & 11,510 & 762,853 & 6,926 & - \\
\hline other red oak & $2,163,418$ & -- & 789 & 16,698 & 39,191 & $1,485,849$ & 53,870 & 1,480 \\
\hline Select hickory & 939,742 & 1,103 & 6,870 & 1,075 & 14,469 & 560,064 & 4,595 & -- \\
\hline other hickory & $1,003,171$ & 2,076 & 675 & -- & 7,323 & 566,174 & 10,340 & -- \\
\hline 8asswood & 253,100 & -- & - & -- & 10,920 & 36,362 & 1,241 & - \\
\hline Beech & 608,945 & -- & 270 & -- & - & 76,896 & - & -- \\
\hline Hard maple & $1,413,239$ & -- & 915 & 1,297 & 5,259 & 186,886 & 3,337 & 3,010 \\
\hline So ft maple & 809,358 & 1,657 & - & 2,231 & 893 & 38,834 & 838 & -- \\
\hline Elm & 329,362 & 1,492 & -- & - & 1,284 & 50,324 & -. & -- \\
\hline Ash & $1,174,946$ & 8,552 & 2,276 & 1,135 & 14,457 & 199,057 & -- & - \\
\hline Sycamore & 920,914 & 3,666 & 1,668 & 9,980 & 1,031 & 77,948 & -- & -- \\
\hline Cottonwood & 587,853 & 5,160 & -- & 43,275 & -- & 16,564 & - & - \\
\hline Willow & 45,497 & - & - & -. & $\cdots$ & - & - & -- \\
\hline Hackberry & 171,147 & -- & -- & - & -- & 13,986 & - & - \\
\hline Aspen & 110,582 & -- & -- & -- & -- & 47,135 & -- & -- \\
\hline 8irch & 31,507 & -- & - & -- & - & 1,141 & -- & - \\
\hline Sweetgum & 222,029 & - & -- & -- & 1,979 & 29,614 & - & -- \\
\hline Tupe lo & 155,667 & -- & 436 & -- & 4,958 & 38,499 & 1,673 & -- \\
\hline 81 ack cherry & 318,703 & -- & - & 3,866 & 2,505 & 61,996 & 6,006 & $\cdots$ \\
\hline Black walnut & 427,405 & -- & -- & - & 2,437 & 73,344 & -- & -- \\
\hline Butternut & 15,368 & -- & -- & - & -- & +- & -- & -- \\
\hline Yellow-poplar & $1,840,013$ & 2,683 & 17,386 & 7,391 & 25,327 & 364,770 & -- & 5,871 \\
\hline Persimmon & 10,011 & -- & -- & - & - & 3,354 & -- & -- \\
\hline Sassafras & 162,154 & -- & -- & 835 & 643 & 34,545 & -- & 1,492 \\
\hline other hardwoods & 168,454 & - & -- & - & - & 29,694 & -- & - \\
\hline Total & $18,607,694$ & 29,879 & 37,694 & 96,728 & 164,946 & $7,086,390$ & 362,257 & 13,447 \\
\hline All species & $19,224,207$ & 186,532 & 94,322 & 204,774 & 296,854 & $7,140,674$ & 362,257 & 16,069 \\
\hline
\end{tabular}

1/International 1/4-inch rule.

(Table 45 continued on next page) 
(Table 45 continued)

\begin{tabular}{|c|c|c|c|c|c|c|c|}
\hline \multirow[b]{2}{*}{ Species group } & \multicolumn{7}{|c|}{ Forest type } \\
\hline & $\begin{array}{l}\text { Oak - } \\
\text { gum }\end{array}$ & $\begin{array}{c}\text { Lowl and } \\
\text { oak }\end{array}$ & $\begin{array}{c}\text { Elm-ash- } \\
\text { soft } \\
\text { maple }\end{array}$ & $\begin{array}{l}\text { Cotton- } \\
\text { wood }\end{array}$ & $\begin{array}{l}\text { Maple- } \\
\text { beech }\end{array}$ & $\begin{array}{l}\text { Cherry-ash- } \\
\text { yel low- } \\
\text { poplar }\end{array}$ & $\begin{array}{c}\text { Non- } \\
\text { stocked }\end{array}$ \\
\hline \multicolumn{8}{|l|}{ Softwoods } \\
\hline Jack pine & $\cdots$ & - & 838 & -- & -- & 5,038 & -- \\
\hline Red pine & -- & -- & -- & -- & -- & -- & -. \\
\hline White pine & -- & -- & 3,778 & -- & -- & 3,932 & -- \\
\hline Shortleaf pine & & -- & 2,579 & -- & -- & 2,539 & -- \\
\hline other yellow pines & 1,424 & -- & 3,029 & -- & 14,156 & 1,823 & - \\
\hline Tamarack & - & -- & 4,208 & -- & -- & -- & $=$ \\
\hline Baldcypress & 5,296 & -- & 36,339 & -- & -- & -- & - \\
\hline Eastern redcedar & - & -- & 1,661 & -- & 10,810 & 8,922 & -- \\
\hline Other softwoods & -- & -- & -- & -- & -- & -- & -- \\
\hline Total & 6,720 & -- & 52,432 & -- & 24,966 & 22,254 & - \\
\hline \multicolumn{8}{|l|}{ Hardwoods } \\
\hline Select white oak & 11,331 & 31,470 & 148,035 & - & 365,697 & 157,992 & 1,093 \\
\hline other white oak & 2,059 & 3,878 & 1,010 & -- & 39,995 & 7,866 & - \\
\hline Select red oak & 7,343 & $\begin{array}{r}976 \\
96\end{array}$ & 52,253 & -- & 326,168 & 141,524 & -- \\
\hline other red oak & 35,838 & 86,430 & 157,943 & -- & 167,248 & 115,764 & 2,318 \\
\hline Select hickory & 8,355 & 1,755 & 77,267 & -- & 207,026 & 57,163 & -- \\
\hline other hickory & -- & 2,886 & 51,402 & -- & 241,169 & 121,126 & -- \\
\hline Basswood & $=-$ & 965 & 27,059 & -- & 159,033 & 17,520 & -- \\
\hline Beech & 990 & -- & 8,980 & -- & 486,404 & 35,405 & -- \\
\hline Hard maple & 903 & & 60,516 & -- & $1,061,816$ & 89,300 & -- \\
\hline Soft maple & 20,944 & 4,683 & 555,291 & 14,420 & 121,994 & 47,573 & -- \\
\hline Elm & 1,668 & - & 99,567 & - & 126,104 & 48,923 & -- \\
\hline Ash & 14,656 & 8,002 & 306,732 & -- & 237,205 & 382,874 & -- \\
\hline Sycamore & 16,032 & -- & 451,796 & 1,119 & 172,570 & 178,401 & 6,703 \\
\hline Cottonwood & - & 3,062 & 401,670 & 83,258 & 12,252 & 19,185 & 3,427 \\
\hline Willow & -- & -- & 44,769 & -- & -- & 728 & - \\
\hline Hackberry & -- & -- & 95,901 & -- & 41,655 & 19,605 &.- \\
\hline Aspen & 2,880 & -. & 4,422 & -- & 17,113 & 39,032 & - \\
\hline Birch & 2,805 & -- & 26,752 & -- & - & 809 & - \\
\hline Sweetgum & 70,981 & 5,649 & 66,919 & -- & 25,443 & 21,444 & -. \\
\hline Tupe lo & 11,599 & 4,619 & 24,088 & -- & 48,592 & 21,203 & -- \\
\hline Black cherry & 2,484 & 2,085 & 23,688 & -- & 136,346 & 79,727 & -. \\
\hline Black walnut & 2,067 & - & 103,811 & -- & 102,410 & 143,336 & -- \\
\hline Butternut & - & -- & 2,871 & -. & 11,428 & 1,069 & -- \\
\hline Yellow-poplar & 23,044 & -- & 159,026 & -- & 419,008 & 815,507 & -. \\
\hline Persimmon & - & - & - & -- & 4,459 & 2,198 & -- \\
\hline Sassafras & 670 & 1,200 & 35,667 & -- & 61,185 & 25,917 & .. \\
\hline other hardwoods & 894 & 978 & 73,399 & 1,750 & 49,879 & 11,860 & $\cdots$ \\
\hline Total & 237,543 & 158,638 & $3,060,834$ & 100,547 & $4,642,199$ & $2,603,051$ & 13,541 \\
\hline All species & 244,263 & 158,638 & $3,113,266$ & 100,547 & $4,667,165$ & $2,625,305$ & 13,541 \\
\hline
\end{tabular}


Table 46.--Net volume of growing stock on timberland by species group and ownership class, Indiana, 1986

(In thousand cubic feet)

\begin{tabular}{|c|c|c|c|c|c|c|c|c|c|}
\hline \multirow[b]{2}{*}{ Species group } & \multirow[b]{2}{*}{$\begin{array}{c}\text { A11 } \\
\text { owners }\end{array}$} & \multicolumn{8}{|c|}{ Ownership class } \\
\hline & & $\begin{array}{l}\text { National } \\
\text { forest }\end{array}$ & $\begin{array}{c}\text { Misc. } \\
\text { federal }\end{array}$ & State & $\begin{array}{l}\text { County \& } \\
\text { municipal }\end{array}$ & $\begin{array}{l}\text { Forest } \\
\text { industry }\end{array}$ & Farmer & $\begin{array}{l}\text { Misc. } \\
\text { priv.- } \\
\text { corp. }\end{array}$ & $\begin{array}{l}\text { Misc. } \\
\text { priv.- } \\
\text { indiv. }\end{array}$ \\
\hline \multicolumn{10}{|l|}{ Softwoods } \\
\hline Jack pine & 6,119 & -- & -- & 1,268 & -- & - & 2,350 & 454 & 2,047 \\
\hline Red pine & 6,763 & -- & -- & 626 & -- & -- & 2,993 & 2,161 & 983 \\
\hline White pine & 39,757 & 2,049 & -- & 4,355 & -- & -- & 8,296 & 10,895 & 14,162 \\
\hline Shortleaf pine & 29,314 & 16,803 & 154 & 4 & - & - & $\begin{array}{l}0,290 \\
3,678\end{array}$ & $\begin{array}{r}10,095 \\
3,331\end{array}$ & $\begin{array}{r}14,102 \\
5,348\end{array}$ \\
\hline other yellow pines & 46,661 & -- & 80 & 6,510 & -- & - & 11,287 & 8,235 & 20,549 \\
\hline Tamarack & 1,585 & -- & -- & 1,585 & -- & -- & -- & -- & -- \\
\hline Baldcypress & 8,788 & -- & -- & -- & -- & -- & $\rightarrow$ & 1,345 & 7,443 \\
\hline Eastern redcedar & 52,586 & 3,418 & 479 & 1,417 & 176 & -- & 24,587 & 3,054 & 19,455 \\
\hline Other softwoods & 9,689 & -- & - & 452 & -- & -- & 9,237 & -- & -- \\
\hline Total & 201,262 & 22,270 & 713 & 16,213 & 176 & -- & 62,428 & 29,475 & 69,987 \\
\hline \multicolumn{10}{|l|}{ Hardwoods } \\
\hline Select white oak & 670,710 & 55,583 & 41,405 & 63,972 & 5,007 & 5,964 & 223,280 & 40,567 & 234,932 \\
\hline other white oak & 123,634 & 19,312 & 2,365 & 29,731 & 1,502 & 798 & 12,095 & 5,842 & 51,989 \\
\hline $\begin{array}{l}\text { Select red oak } \\
\text { other red oak }\end{array}$ & $\begin{array}{l}291,878 \\
497,322\end{array}$ & 11,470 & 10,106 & 16,556 & 1,494 & 2,190 & 101,710 & 31,909 & 116,443 \\
\hline $\begin{array}{l}\text { other red oak } \\
\text { Select hickory }\end{array}$ & $\begin{array}{l}49 /, 322 \\
274,705\end{array}$ & $\begin{array}{r}31,2 / 9 \\
9,611\end{array}$ & $\begin{array}{l}27,596 \\
12,379\end{array}$ & $\begin{array}{r}44,620 \\
6,721\end{array}$ & $\begin{array}{r}1,382 \\
910\end{array}$ & $\begin{array}{l}3,493 \\
1,343\end{array}$ & $\begin{array}{l}163,599 \\
107,449\end{array}$ & $\begin{array}{l}42,037 \\
20,200\end{array}$ & $\begin{array}{l}183,310 \\
116,092\end{array}$ \\
\hline other hickory & 299,253 & 16,934 & 14,937 & 13,052 & 516 & 2,627 & 114,339 & 17,543 & 119,305 \\
\hline Basswood & 67,070 & 142 & 2,805 & 3,549 & 277 & & 28,712 & 6,751 & 24,834 \\
\hline Beech & 145,972 & 4,033 & 5,144 & 5,547 & & 2,787 & 60,132 & 14,789 & 53,540 \\
\hline Hard maple & 454,732 & 15,277 & 16,610 & 9,619 & 1,241 & 3,100 & 181,452 & 44,123 & 183,310 \\
\hline $\begin{array}{l}\text { Soft maple } \\
\text { Elm }\end{array}$ & $\begin{array}{l}243,760 \\
174,074\end{array}$ & $\begin{array}{l}3,023 \\
1,373\end{array}$ & $\begin{array}{r}12,784 \\
1,785\end{array}$ & $\begin{array}{r}13,003 \\
3,511\end{array}$ & $\begin{array}{l}3,171 \\
1,636\end{array}$ & 745 & $\begin{array}{r}128,024 \\
82,508\end{array}$ & $\begin{array}{l}15,266 \\
22,092\end{array}$ & $\begin{array}{l}68,489 \\
60,424\end{array}$ \\
\hline Ash & $\begin{array}{l}174,074 \\
359,677\end{array}$ & 9,803 & $\begin{array}{l}1,103 \\
7,914\end{array}$ & 10,764 & $\begin{array}{l}1,030 \\
3,475\end{array}$ & $\begin{array}{l}143 \\
-.\end{array}$ & $\begin{array}{r}156,898 \\
156,500\end{array}$ & 36,338 & 134,485 \\
\hline Sycamore & 219,745 & 6,784 & 8,924 & 6,081 & 830 & -- & 96,313 & 20,186 & 80,627 \\
\hline Cottonwood & 129,300 & -- & 2,245 & 1,907 & 8,656 & -- & 78,220 & 15,321 & 22,951 \\
\hline Willow & 13,501 & -- & -- & -- & 313 & -- & 3,555 & 618 & 9,015 \\
\hline Hackberry & 51,859 & -- & 543 & 628 & 208 & -- & 23,660 & 4,211 & 22,609 \\
\hline Aspen & 31,290 & 2,496 & 2,907 & 153 & - & $\rightarrow$ & 10,535 & 3,997 & 11,202 \\
\hline Birch & 12,538 & 55 & 317 & 926 & -- & -- & 5,351 & 958 & 4,931 \\
\hline Sweetgum & 62,099 & 1,108 & 989 & 3,064 & -- & - & 26,483 & 4,626 & 25,829 \\
\hline Tupe 10 & 46,152 & 2,699 & 1,150 & 3,504 & -- & 340 & 17,806 & 2,615 & 18,038 \\
\hline Bl ack cherry & 102,003 & 2,255 & 2,924 & 3,343 & 1,875 & - & 43,590 & 9,707 & 38,309 \\
\hline Black walnut & 127,229 & 475 & 4,581 & 2,384 & 994 & 346 & 61,561 & 12,378 & 44,510 \\
\hline Butternut & 3,981 & -- & -- & -- & 783 & -- & 2,123 & 585 & 490 \\
\hline Yellow-poplar & 432,602 & 17,209 & 22,547 & 17,411 & 582 & 921 & 154,595 & 32,255 & 187,082 \\
\hline Persimmon & 12,010 & 112 & 420 & 1,096 & -- & -- & 3,601 & 1,460 & 5,321 \\
\hline Sassafras & 97,800 & 4,576 & 2,058 & 4,610 & -- & 1,830 & 29,143 & 7,368 & 48,215 \\
\hline other hardwoods & 71,696 & 946 & 3,356 & 564 & 1,304 & $\ldots$ & 27,600 & 6,235 & 31,691 \\
\hline Total & $5,016,592$ & 216,555 & 208,791 & 266,322 & 36,156 & 26,484 & $1,944,334$ & 419,977 & $1,897,973$ \\
\hline All species & $5,217,854$ & 238,825 & 209,504 & 282,535 & 36,332 & 26,484 & $2,006,762$ & 449,452 & $1,967,960$ \\
\hline
\end{tabular}


Table 47.--Net volume of sawtimber on timberland by species group and ownership class, Indiana, 1986 (In thousand board feet) $\underline{\underline{1}}$ '

\begin{tabular}{|c|c|c|c|c|c|c|c|c|c|}
\hline \multirow[b]{2}{*}{ Species group } & \multirow[b]{2}{*}{$\begin{array}{c}\text { All } \\
\text { owners }\end{array}$} & \multicolumn{8}{|c|}{ Ownership class } \\
\hline & & $\begin{array}{c}\text { National } \\
\text { forest }\end{array}$ & $\begin{array}{l}\text { Misc. } \\
\text { federal }\end{array}$ & State & $\begin{array}{l}\text { County \& } \\
\text { municipal }\end{array}$ & $\begin{array}{c}\text { Forest } \\
\text { industry }\end{array}$ & Farmer & $\begin{array}{l}\text { Misc. } \\
\text { priv.- } \\
\text { corp. }\end{array}$ & $\begin{array}{l}\text { Misc. } \\
\text { priv.- } \\
\text { indiv. }\end{array}$ \\
\hline $\begin{array}{l}\text { So ftwoods } \\
\text { Jack pine } \\
\text { Red pine } \\
\text { White pine } \\
\text { Shortleaf pine } \\
\text { Other yellow pines } \\
\text { Tamarack } \\
\text { 8aldcypress } \\
\text { Eastern redcedar } \\
\text { Other softwoods }\end{array}$ & $\begin{array}{r}18,409 \\
11,919 \\
151,387 \\
87,129 \\
183,341 \\
4,208 \\
41,635 \\
113,761 \\
4,724 \\
\end{array}$ & $\begin{array}{r}-- \\
-- \\
7,729 \\
43,459 \\
-- \\
-- \\
-- \\
11,072 \\
--\end{array}$ & $\begin{array}{r}-- \\
-- \\
-- \\
840 \\
-- \\
- \\
-- \\
1,019 \\
--\end{array}$ & $\begin{array}{r}5,038 \\
2,034 \\
18,080 \\
-\ldots \\
25,739 \\
4,208 \\
-- \\
7,897 \\
--\end{array}$ & $\begin{array}{l}-- \\
-- \\
-- \\
-- \\
-- \\
-- \\
-- \\
-- \\
--\end{array}$ & $\begin{array}{l}-- \\
-- \\
-- \\
-- \\
-- \\
-- \\
-- \\
-- \\
--\end{array}$ & $\begin{array}{r}7,001 \\
5,127 \\
33,407 \\
11,684 \\
45,962 \\
-- \\
-- \\
34,799 \\
4,724 \\
\end{array}$ & $\begin{array}{r}-- \\
2,400 \\
49,426 \\
15,958 \\
19,913 \\
-- \\
6,766 \\
15,101 \\
-- \\
\end{array}$ & $\begin{array}{r}6,370 \\
2,358 \\
42,745 \\
15,188 \\
91,727 \\
\ldots \\
34,869 \\
43,873 \\
.-\end{array}$ \\
\hline Total & 616,513 & 62,260 & 1,859 & 62,996 & -- & - & 142,704 & 109,564 & 237,130 \\
\hline $\begin{array}{l}\text { Hardwoods } \\
\text { Select white oak } \\
\text { Other white oak } \\
\text { Select red oak } \\
\text { Other red oak } \\
\text { Select hickory } \\
\text { Other hickory } \\
\text { 8asswood } \\
\text { Beech } \\
\text { Hard maple } \\
\text { Soft maple } \\
\text { Elm } \\
\text { Ash } \\
\text { Sycamore } \\
\text { Cottonwood } \\
\text { Willow } \\
\text { Hackberry } \\
\text { Aspen } \\
\text { Birch } \\
\text { Sweetgum } \\
\text { Tupelo } \\
\text { Black cherry } \\
\text { Black walnut } \\
\text { 8utternut } \\
\text { Yellow-poplar } \\
\text { Persimmon } \\
\text { Sassafras } \\
\text { Other hardwoods }\end{array}$ & $\begin{array}{r}2,875,793 \\
533,134 \\
1,316,182 \\
2,163,418 \\
939,742 \\
1,003,171 \\
253,100 \\
608,945 \\
1,413,239 \\
809,358 \\
329,362 \\
1,174,946 \\
920,914 \\
587,853 \\
45,497 \\
171,147 \\
110,582 \\
31,507 \\
222,029 \\
155,667 \\
318,703 \\
427,405 \\
15,368 \\
1,840,013 \\
10,011 \\
162,154 \\
168,454\end{array}$ & $\begin{array}{r}226,466 \\
83,313 \\
47,465 \\
129,778 \\
36,520 \\
45,912 \\
-- \\
12,122 \\
38,545 \\
6,674 \\
718 \\
18,139 \\
12,979 \\
-- \\
-- \\
-- \\
8,509 \\
124 \\
4,846 \\
8,275 \\
6,066 \\
2,460 \\
-- \\
74,366 \\
-- \\
1,925 \\
4,080\end{array}$ & $\begin{array}{r}191,806 \\
11,324 \\
40,269 \\
124,916 \\
38,785 \\
44,893 \\
11,088 \\
16,963 \\
45,865 \\
33,394 \\
2,328 \\
18,528 \\
38,186 \\
9,919 \\
-- \\
2,796 \\
12,676 \\
903 \\
4,560 \\
909 \\
6,069 \\
12,701 \\
-- \\
97,073 \\
-- \\
2,732 \\
7,014\end{array}$ & $\begin{array}{r}262,461 \\
128,435 \\
72,738 \\
198,132 \\
20,670 \\
44,506 \\
14,881 \\
26,647 \\
14,923 \\
44,161 \\
8,687 \\
34,313 \\
22,595 \\
5,591 \\
-- \\
1,867 \\
-- \\
1,862 \\
9,837 \\
12,202 \\
13,003 \\
7,357 \\
-- \\
73,681 \\
-- \\
1,263 \\
835 \\
\end{array}$ & $\begin{array}{r}23,915 \\
6,981 \\
7,456 \\
6,068 \\
4,717 \\
676 \\
1,422 \\
-- \\
6,222 \\
10,888 \\
1,717 \\
5,432 \\
3,025 \\
40,541 \\
-- \\
1,067 \\
-- \\
-- \\
-- \\
-- \\
3,188 \\
4,358 \\
4,008 \\
1,896 \\
-- \\
-- \\
-- \\
\end{array}$ & $\begin{array}{r}23,830 \\
2,279 \\
9,787 \\
12,854 \\
4,663 \\
12,858 \\
-- \\
14,463 \\
12,381 \\
-- \\
821 \\
-- \\
-- \\
-. \\
-- \\
-- \\
-- \\
-. \\
-- \\
1,449 \\
-- \\
876 \\
-. \\
3,544 \\
-- \\
4,048 \\
-- \\
\end{array}$ & $\begin{array}{r}971,010 \\
52,526 \\
465,724 \\
706,901 \\
364,987 \\
386,034 \\
107,267 \\
254,479 \\
628,041 \\
450,388 \\
155,771 \\
529,626 \\
424,873 \\
365,909 \\
5,836 \\
69,862 \\
38,430 \\
16,027 \\
98,475 \\
59,115 \\
132,963 \\
210,600 \\
7,420 \\
662,235 \\
2,297 \\
47,035 \\
70,559 \\
\end{array}$ & $\begin{array}{r}182,706 \\
23,943 \\
146,745 \\
189,900 \\
69,206 \\
65,589 \\
26,630 \\
64,954 \\
129,204 \\
46,128 \\
48,169 \\
118,473 \\
65,644 \\
55,574 \\
-- \\
15,279 \\
14,068 \\
-- \\
10,799 \\
9,260 \\
30,960 \\
46,971 \\
2,871 \\
125,687 \\
2,856 \\
16,592 \\
10,668 \\
\end{array}$ & $\begin{array}{r}993,599 \\
224,333 \\
525,998 \\
794,869 \\
400,194 \\
402,703 \\
91,812 \\
219,317 \\
538,058 \\
217,725 \\
111,151 \\
450,435 \\
353,612 \\
110,319 \\
39,661 \\
80,276 \\
36,899 \\
12,591 \\
93,512 \\
64,457 \\
126,454 \\
142,082 \\
1,069 \\
801,531 \\
4,858 \\
88,559 \\
75,298 \\
\end{array}$ \\
\hline Tota 1 & $18,607,694$ & 769,282 & 775,697 & $1,020,647$ & 133,577 & 103,853 & $7,284,390$ & $1,518,876$ & $7,001,372$ \\
\hline All species & $19,224,207$ & 831,542 & 777,556 & $1,083,643$ & 133,577 & 103,853 & $7,427,094$ & $1,628,440$ & $7,238,502$ \\
\hline
\end{tabular}

1/International $1 / 4$-inch rule. 


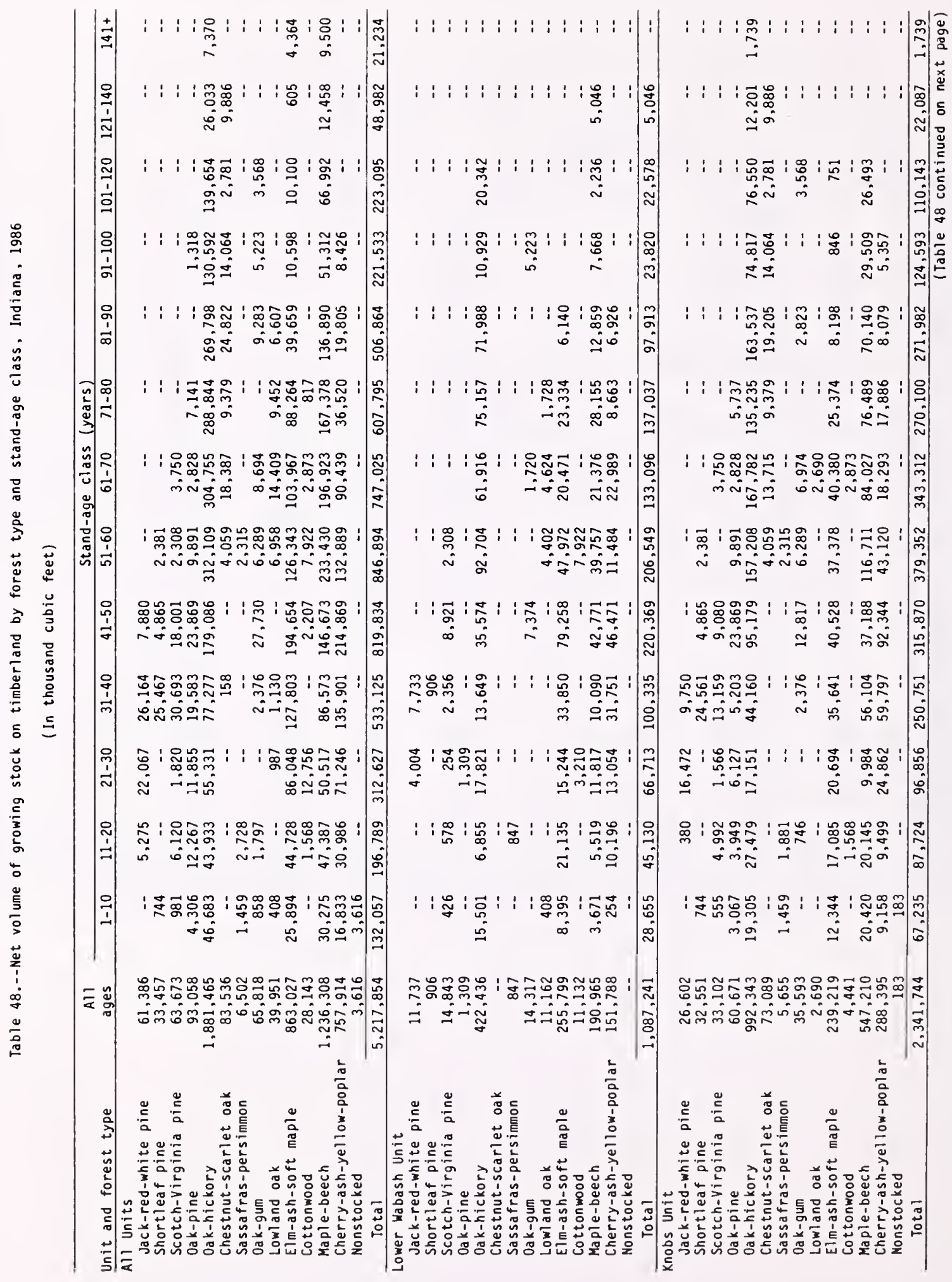




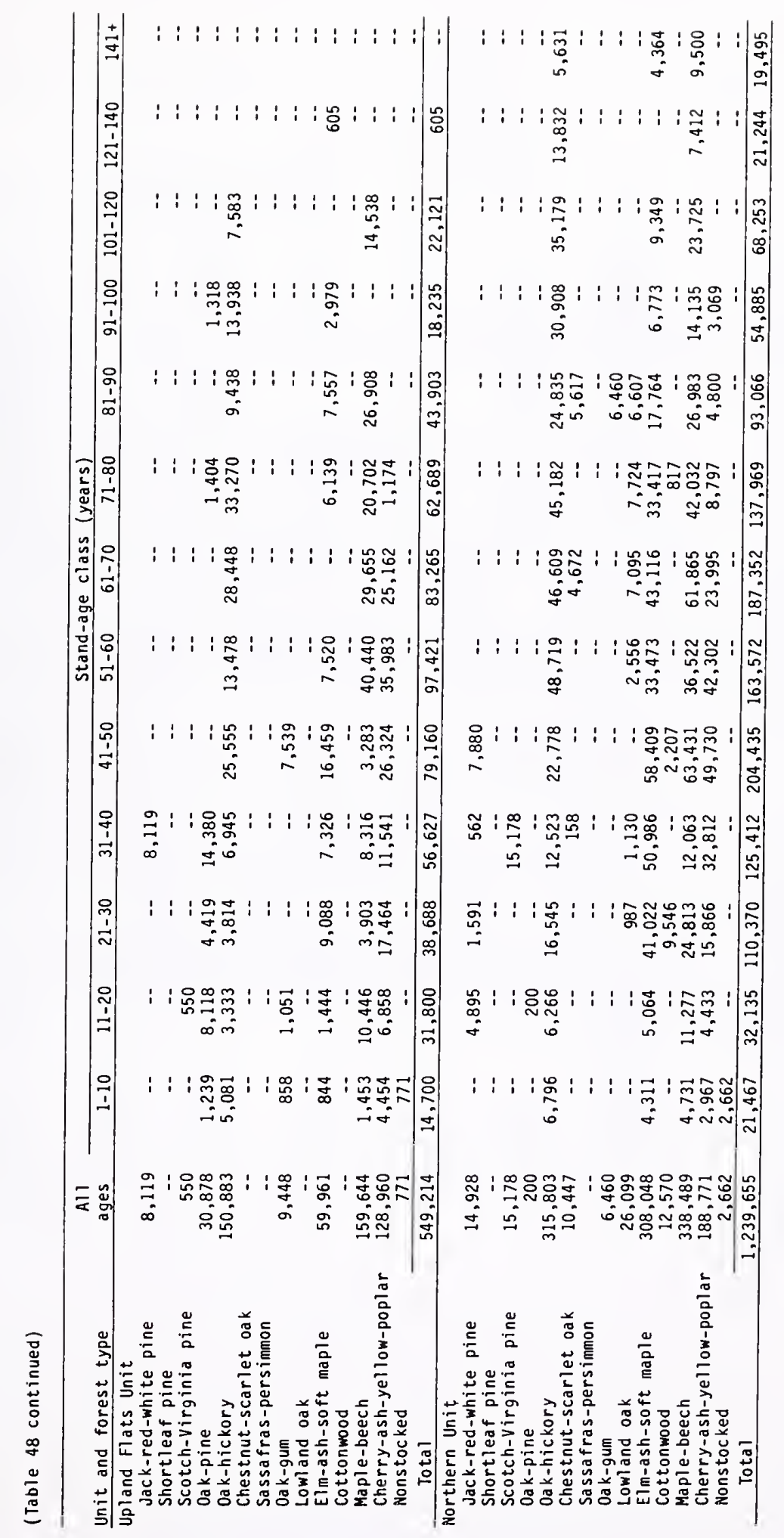


Table 49.--Net volume of sawtimber on timberland by forest type and stand-age class, Indiana, 1986

(In thousand board feet) ${ }^{1 /}$

\begin{tabular}{|c|c|c|c|c|c|c|c|c|}
\hline \multirow[b]{2}{*}{ Unit and forest type } & \multirow{2}{*}{$\begin{array}{r}\text { All } \\
\text { ages }\end{array}$} & \multicolumn{7}{|c|}{ Stand-age class (years) } \\
\hline & & $1-10$ & $11-20$ & $21-30$ & $31-40$ & $41-50$ & $51-60$ & $61-70$ \\
\hline \multicolumn{9}{|l|}{ All Units } \\
\hline Jack-red-white pine & 186,532 & -- & 10,261 & 59,495 & 86,894 & 29,882 & - & -- \\
\hline Shortleaf pine & & 1,897 & & & 64,006 & 17,635 & 10,784 & -- \\
\hline Scotch-Virginia pine & 204,774 & $\cdots$ & 12,929 & 1,656 & 94,652 & 71,823 & 7,010 & 16,704 \\
\hline 0ak-pine & 296,854 & 9,205 & 38,607 & 17,954 & 55,803 & 95,286 & 41,561 & 15,044 \\
\hline Oak-hickory & $7,140,674$ & 154,929 & 118,979 & 136,933 & 201,876 & 614,746 & $1,143,155$ & $1,186,182$ \\
\hline Chestnut-scarlet oak & 362,257 & & & -- & 493 & -- & 17,314 & 77,736 \\
\hline Sassafras-persimmon & 16,069 & 5,696 & 2,485 & -- & -- & -- & 7,888 & -- \\
\hline Oak-gum & 244,263 & 3,703 & 4,873 & -- & 3,662 & 103,265 & 30,056 & 34,998 \\
\hline Lowland oak & 158,638 & 1,990 & -- & -- & 3,681 & -- & 31,345 & 60,667 \\
\hline Elm-ash-soft maple & $3,113,266$ & 61,130 & 122,552 & 234,502 & 402,317 & 730,049 & 494,910 & 431,643 \\
\hline Cottonwood & 100,547 & & 801 & 37,293 & & 11,075 & 34,636 & 13,538 \\
\hline Maple-beech & $4,667,165$ & 79,022 & 121,294 & 115,164 & 251,405 & 580,452 & 903,930 & 790,170 \\
\hline Cherry-ash-yellow-poplar & $2,625,305$ & 53,154 & 66,630 & 178,728 & 461,012 & 787,693 & 482,213 & 340,685 \\
\hline Nonstocked & 13,541 & 13,541 & $\ldots$ & -- & $\cdots$ & $\ldots$ & _.. & -.- \\
\hline Total & $19,224,207$ & 384,267 & 499,411 & 781,725 & $1,625,801$ & $3,041,906$ & $3,204,802$ & $2,967,367$ \\
\hline \multicolumn{9}{|l|}{ Lower Wabash Unit } \\
\hline Jack-red-whi & 38,863 & - & -- & 12,982 & 25,881 & -- & -- & -- \\
\hline Shortleaf $p$ & 2,041 & -- & -- & -- & 2,041 & -. & -. & -- \\
\hline lirginia pine & 41,424 & -- & 1,131 & -- & 4,960 & 28,323 & 7,010 & -- \\
\hline Oak- & 4,093 & - & & 4,093 & & & & $\because$ \\
\hline $0 a k-h$ & $1,645,880$ & .438 &, 756 & 47,941 & 39,267 & 124,612 & 364,829 & 254,137 \\
\hline carlet oak & -- & -- & -- & -- & -- & -- & -- & -- \\
\hline Sassafras-persimmon & -- & -- & -. & -- & -- & -- & -- & -. \\
\hline Oak-gum & 48,658 & -- & -- & -- & -- & 22,613 & -- & 7,456 \\
\hline Lowl and oa & 51,044 & 1,990 & -- & -- & -- & & 21,247 & 20,758 \\
\hline Elm-ash-soft maple & 941,337 & 19,948 & 56,315 & 41,909 & 115,432 & 309,262 & 188,065 & 94,933 \\
\hline Cottonwood & 38,226 & -- & & 3,590 & & & 34,636 & -- \\
\hline Maple-beech & 674,013 & 9,652 & 15,691 & 26,249 & 27,583 & 159,597 & 132,593 & 74,830 \\
\hline Cherry-ash-yellow-poplar & 541,189 & -- & 23,278 & 53,764 & 113,671 & 176,716 & 40,993 & 78,481 \\
\hline Nons toc ked & & -- & -- & $\because$ & $\because$ & -- & -- & $\ldots$ \\
\hline Total & $4,026,768$ & 87,028 & 112,171 & 190,528 & 328,835 & 821,123 & 789,373 & 530,595 \\
\hline \multicolumn{9}{|l|}{ Knobs Unit } \\
\hline Jack-red-wh & 77,572 & -- & -- & 43,542 & 34,030 & -- & -- & -- \\
\hline Shortleaf $p$ & & 1,897 & & & 61,965 & 17,635 & 10,784 & -. \\
\hline Scotch-Virg & & & 900 & 1,656 & 45,797 & 43,500 & -- & 16,704 \\
\hline Oak-pine & 216,517 & 8,283 & 973 & 10,180 & 15,898 & 95,286 & 41,561 & 15,044 \\
\hline Oak &, 738 & 62,080 & 74,908 & 39,952 & 132,672 & 338,263 & 538,643 & 640,013 \\
\hline Chestnut-scarlet oak & 315,322 & -- & -- & -. & -- & -- & 17,314 & 58,968 \\
\hline Sassafras-persimmon & & 5,696 & 2,485 & -- & -- & -- & 7,888 & -- \\
\hline Oak & & -- & 3,839 & -- & 3,662 & 49,111 & 30,056 & 27,542 \\
\hline Lon & & -- & & -- &.- & -- & -. & 11,516 \\
\hline Elm-ash-soft maple & 846,568 & 29,605 & 49,836 & 41,582 & 117,446 & 142,462 & 151,224 & 170,024 \\
\hline Cottc & 330 & & 801 & -- & & -- & & 13,538 \\
\hline Maple-beech & $2,081,174$ & 46,629 & 52,877 & 15,040 & 689 & 144,470 & 466,332 & 346,026 \\
\hline Cherr & $1,022,822$ & 144 & 20,906 & 6,455 & 98,190 & 355,794 & 158,442 & 68,866 \\
\hline Nons tocked & & -- & -- & -- & -- & -- & - & \\
\hline Total & $8,688,845$ & 184,334 & 231,525 & 218,407 & 783,349 & $1,186,521$ & $1,422,244$ & $1,368,241$ \\
\hline
\end{tabular}


(Table 49 continued)

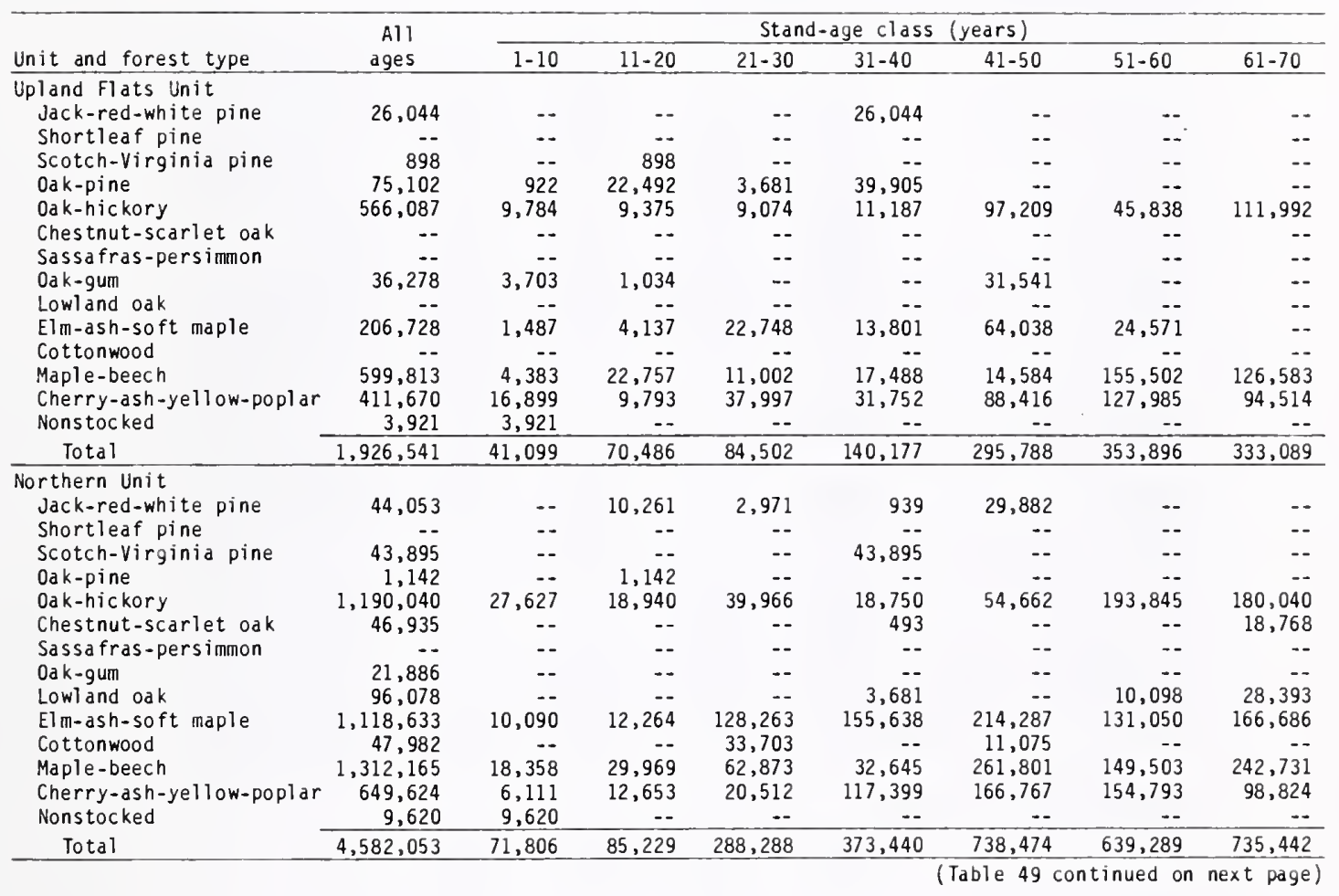


(Table 49 continued)

\begin{tabular}{|c|c|c|c|c|c|c|}
\hline \multirow[b]{2}{*}{ Unit and forest type } & \multicolumn{6}{|c|}{ Stand-age class (years) } \\
\hline & $71-80$ & $81-90$ & $91-100$ & $101-120$ & $121-140$ & $141+$ \\
\hline \multicolumn{7}{|l|}{ All Units } \\
\hline Jack-red-white pine & -- & -- & -- & -- & -- & -- \\
\hline Shortleaf pine & -- & -- & -- & -- & -- & -- \\
\hline Scotch-Virginia pine & -- & -- & -- & -- & -- & -- \\
\hline oak-pine & 18,945 & -- & 4,449 & -- & - & -- \\
\hline Oak-hickory & $1,174,673$ & $1,115,503$ & 538,470 & 604,004 & 118,792 & 32,432 \\
\hline Chestnut-scarlet oak & 40,987 & 111,208 & 61,275 & 11,201 & 42,043 & -- \\
\hline Sassafras-persimmon & - & -- & -- & -- & -- & -- \\
\hline Oak-gum & -- & 32,598 & 18,589 & 12,519 & -- & - \\
\hline Lowl and oak & 40,259 & 20,696 & - & -- & -- & -- \\
\hline Elm-ash-soft maple & 340,109 & 177,578 & 49,198 & 45,987 & 3,063 & 20,228 \\
\hline Cottonwood & 3,204 & & & - & $-\overline{-1}$ & \\
\hline Maple-beech & 678,821 & 545,424 & 218,480 & 291,071 & 47,487 & 44,445 \\
\hline Cherry-ash-yellow-poplar & 139,622 & 83,524 & 32,044 & - & -. & -. \\
\hline Nonstocked & -- & -- & -- & $\because$ & $\cdots$ & - \\
\hline Total & $2,436,620$ & $2,086,531$ & 922,505 & 964,782 & 211,385 & 97,105 \\
\hline \multicolumn{7}{|l|}{ Lower Wabash Unit } \\
\hline Jack-red-white pine & -- & -- & -- & -- & -- & - \\
\hline Shortleaf pine & - & $\cdots$ & $=-$ & $\cdots$ & - & - \\
\hline Scotch-Virginia pine & -- & -- & -- & -- & -- & -- \\
\hline oak-pine & -- & -- & -- & -- & -- & $\cdots$ \\
\hline Oak-hickory & 307,069 & 309,538 & 40,501 & 86,792 & -- & - \\
\hline Chestnut-scarlet oak & -. & -- & -- & - & -- & -- \\
\hline Sassafras-persimmon & -- & -- & -- & -- & -- & - \\
\hline oak-gum & -- & -- & 18,589 & -- & - & 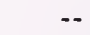 \\
\hline Lowl and oak & 7,049 & & -- & -- & -- & - \\
\hline Elm-ash-soft maple & 93,462 & 22,011 & -- & -- & -- & -- \\
\hline Cottonwood & & -- & $=$ & -- & & -- \\
\hline Maple-beech & 110,837 & 55,887 & 32,407 & 10,452 & 18,235 & -- \\
\hline Cherry-ash-yellow-poplar & 27,466 & 820 & -- & -- & -- & -- \\
\hline Nons tocked & -- & -- & -- & -- & -- & -- \\
\hline Total & 545,883 & 4,256 & 91,497 & 97,244 & 18,235 & $\cdots$ \\
\hline \multicolumn{7}{|l|}{ Knobs Unit } \\
\hline Jack-red-white pine & -- & -- & -- & - & -- & - \\
\hline rtleaf pine & -- & -- & -- & - & - & -- \\
\hline Scotch-Virginia pine & $\therefore$ & -- & $\cdots$ & -- & - & - \\
\hline Oak-pine & 15,292 & -- & -- & - & $\cdots$ & - \\
\hline Oak-hickory & 547,781 & 671,456 & 300,587 & 329,056 & 428 & 6,828 \\
\hline carlet oak & 40,987 & 83,534 & 61,275 & 11,201 & 043 & - \\
\hline Sassafras-persimmon & -- & -- & -- & -- & $\cdots$ & $\cdots$ \\
\hline 0ak-gum & -- & 10,712 & -- & 12,519 & -- & -- \\
\hline Lowl and oak & -- & -- & -- & -- & -- & -- \\
\hline Elm-ash-soft maple & 98,288 & 39,750 & 3,098 & 3,253 & -- & -- \\
\hline Cottonwood & -- & -- & -- & -. & -- & - \\
\hline Maple-beech & 316,092 & 284,759 & 124,267 & 110,993 & -- & $\cdots$ \\
\hline Cherry-ash-yellow-poplar & 69,527 & 35,172 & 19,326 & -- & -- & -- \\
\hline Nonstocked & -- & -- & -- & -- & -- & - \\
\hline Total & $1,087,967$ & $1,125,383$ & 508,553 & 467,022 & 98,471 & 6,828 \\
\hline
\end{tabular}


(Table 49 continued)

\begin{tabular}{|c|c|c|c|c|c|c|}
\hline \multirow[b]{2}{*}{ Unit and forest type } & \multicolumn{6}{|c|}{ Stand-age class (years) } \\
\hline & $71-80$ & $81-90$ & $91-100$ & $101-120$ & $121-140$ & $141+$ \\
\hline \multicolumn{7}{|l|}{ Upland Flats Unit } \\
\hline Jack-red-wh & -- & -- & -- & -- & -- & -- \\
\hline Shortleaf pine & -- & -- & -- & -- & -- & -- \\
\hline Scotch-Virginia pine & -- & -- & $\cdots$ & -- & -- & -- \\
\hline Oak-pine & 3,653 & -- & 4,449 & -- & -- & -- \\
\hline Oak-hickory & 139,495 & 37,856 & 60,606 & 33,671 & $-\cdot$ & -- \\
\hline t oak & -. & -- & - & -- & -- & -- \\
\hline Sassafras-persimmon & -- & -- & -- & $\cdots$ & -- & -- \\
\hline oak & -- & -- & -- & -- & -- & - \\
\hline Low1 & -- & -- & -- & -- & -- & -- \\
\hline -soft maple & 26,694 & 32,352 & 13,837 & -- & 3,063 & $=-$ \\
\hline Cottonwood & & -- & -- & -- & -- & -- \\
\hline Maple- & 75,849 & 105,722 & -- & 65,943 & -- & -- \\
\hline Cherry-ash-yellow-poplar & 4,314 & -. & -- & -- & -- & -- \\
\hline Nonstocked & -- & -- & -- & -- & -- & -- \\
\hline Total & 50,005 & 175,930 & 78,892 & 99,614 & 3,063 & $=$ \\
\hline \multicolumn{7}{|l|}{ Northern Unit } \\
\hline נ] & -- & -- & -- & -- & -- & -- \\
\hline Shortl & -- & -- & -- & -- & -- & -- \\
\hline Scotch-Virginia pine & -- & -- & -- & -- & -- & -- \\
\hline oak- & -- & -- & -- & -- & -- & -- \\
\hline kory & 180,328 & 96,653 & 136,776 & 154,485 & 62,364 & 25,604 \\
\hline Ches & -- & 27,674 & -- & -- & -- & - \\
\hline Sassafras-persimmon & -- & - & -- & -- & -- & -- \\
\hline 0ak-gum & -- & 21,886 & -- & -- & -- & -- \\
\hline Lowl and oak & 33,210 & 20,696 & -- & -- & -- & - \\
\hline Elm-ash-soft maple & 121,665 & 83,465 & 32,263 & 42,734 & -- & 20,228 \\
\hline Cottonwood & 3,204 & & & & & \\
\hline Maple-beech & 176,043 & 99,056 & 61,806 & 103,683 & 29,252 & 44,445 \\
\hline Cherry-ash-yellow-poplar & 38,315 & 21,532 & 12,718 & -- & -- & -- \\
\hline Nonstocked & -- & -- & -- & $\because$ & -- & $\because$ \\
\hline Total & 552,765 & 370,962 & 243,563 & 300,902 & 91,616 & 90,277 \\
\hline
\end{tabular}




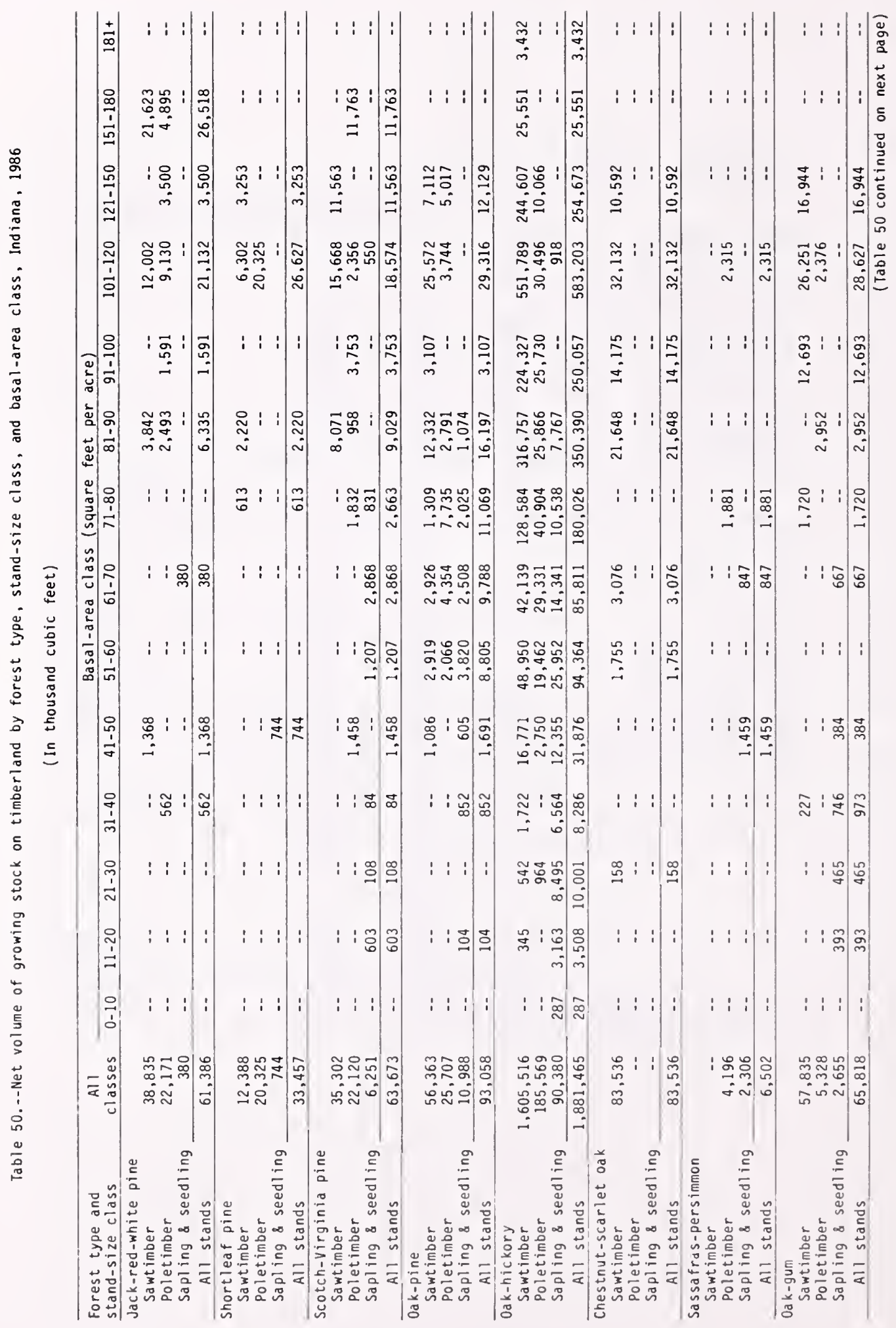




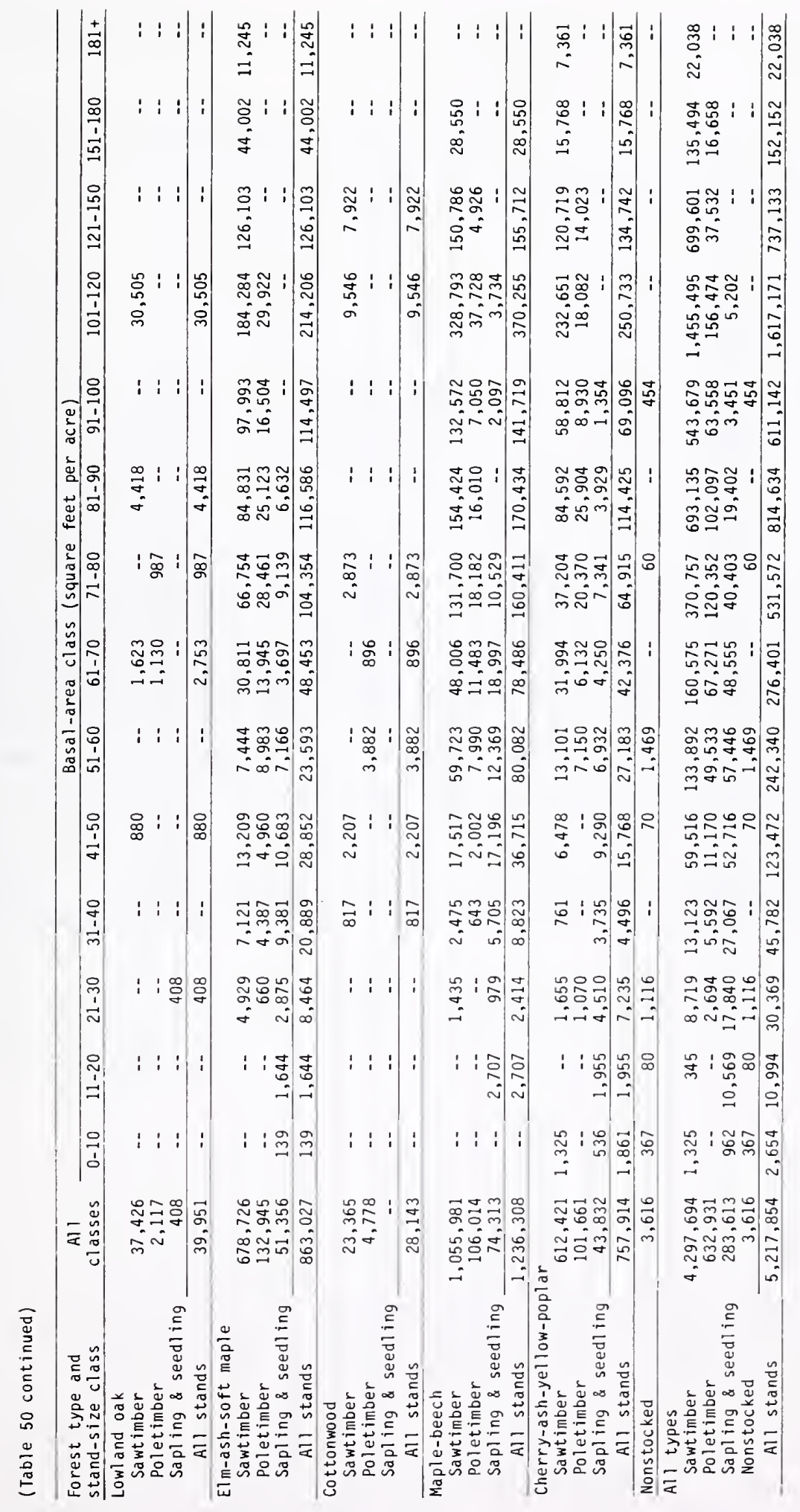


Table 51.--Net volume of sawtimber on timberland by forest type, stand-size class, and basal-area class, Indiana, 1986

(In thousand board feet) 1 /

\begin{tabular}{|c|c|c|c|c|c|c|c|c|c|}
\hline \multirow{2}{*}{$\begin{array}{l}\text { Forest type and } \\
\text { stand-size class }\end{array}$} & \multirow{2}{*}{$\begin{array}{c}\text { All } \\
\text { classes }\end{array}$} & \multicolumn{8}{|c|}{ Basal area class (square feet per acre) } \\
\hline & & $0-10$ & $11-20$ & $21-30$ & $31-40$ & $41-50$ & $51-60$ & $61-70$ & $71-80$ \\
\hline \multicolumn{10}{|l|}{ Jack-red-white pine } \\
\hline Sawtimber & 143,529 & -- & -- & -- & -- & 6,036 & -- & -- & -- \\
\hline Poletinaber & 43,003 & -- & -- & -- & 939 & -- & -- & -- & -- \\
\hline Sapling \& seedling & & -- & -- & -- & -- & -- & -- & -- & -- \\
\hline All stands & 186,532 & -- & -- & -- & 939 & 6,036 & -- & -- & -. \\
\hline \multicolumn{10}{|l|}{ Shortleaf pine } \\
\hline Sawtimber & 46,294 & -- & -- & -- & -- & -- & -- & -- & 2,509 \\
\hline Poletimber & 46,131 & -- & -- & -- & -- & -- & -- & -- & -. \\
\hline Sapling \& seedling & 1,897 & -- & -- & -- & -- & 1,897 & -- & -- & - \\
\hline All stands & 94,322 & -- & -- & -- & -- & 1,897 & -- & -- & 2,509 \\
\hline \multicolumn{10}{|l|}{ Scotch-Virginia pine } \\
\hline \multirow{3}{*}{$\begin{array}{l}\text { Sawtimber } \\
\text { Polet imber } \\
\text { Sapling \& seedling }\end{array}$} & 138,901 & -- & -- & -- & -- & -. & -- & -- & -- \\
\hline & 52,966 & -- & -- & -- & -- & 1,099 & & & 6,864 \\
\hline & 12,907 & -- & 1,223 & 557 & -- & -- & 1,131 & 9,098 & -. \\
\hline All stands & 204,774 & -- & 1,223 & 557 & -- & 1,099 & 1,131 & 9,098 & 6,864 \\
\hline \multicolumn{10}{|l|}{ oak-pine } \\
\hline \multirow{3}{*}{$\begin{array}{l}\text { Sawt imber } \\
\text { Poletimber } \\
\text { Sapling \& seedling }\end{array}$} & 216,546 & -- & -- & -- & -- & 3,939 & 9,945 & 8,661 & 4,093 \\
\hline & 53,949 & -- & -- & -- & -- & -- & 2,642 & 6,383 & 17,021 \\
\hline & 26,359 & $\ldots$ & 539 & -- & 1,142 & -- & 13,902 & 8,815 & \\
\hline All stands & 296,854 & -- & 539 & -- & 1,142 & 3,939 & 26,489 & 23,859 & 21,114 \\
\hline \multicolumn{10}{|l|}{ Oak-hickory } \\
\hline \multirow{4}{*}{$\begin{array}{l}\text { Sawtimber } \\
\text { Poletimber } \\
\text { Sapling \& seedling } \\
\text { All stands }\end{array}$} & $6,483,936$ & -- & 1,063 & 1,533 & 8,018 & 65,353 & 196,577 & 155,277 & 508,292 \\
\hline & 383,685 & -- & & 1,133 & & 5,709 & 38,456 & 68,028 & 80,069 \\
\hline & 273,053 & 769 & 10,853 & 23,129 & 20,537 & 29,560 & 80,324 & 42,166 & 40,263 \\
\hline & $7,140,674$ & 769 & 11,916 & 25,795 & 28,555 & 100,622 & 315,357 & 265,471 & 628,624 \\
\hline \multirow{5}{*}{$\begin{array}{l}\text { Chestnut-scarlet oak } \\
\text { Sawt imber } \\
\text { Poletimber } \\
\text { Sapling \& seedling } \\
\text { All stands }\end{array}$} & & & & & & & & & \\
\hline & 362,257 & -- & -- & 493 & -- & -- & 5,167 & 13,284 & -- \\
\hline & -- & -- & -- & -- & -- & -- & -- & -. & -- \\
\hline & -- & -- & -- & -- & -- & -- & -- & -- & -- \\
\hline & 362.257 & -- & $\because$ & 493 & -- & $\ldots$ & 5,167 & 13,284 & - \\
\hline \multirow{5}{*}{$\begin{array}{l}\text { Sassa fras-persimmon } \\
\text { Sawtimber } \\
\text { Poletimber } \\
\text { Sapl ing \& seedling } \\
\text { All stands }\end{array}$} & & & & & & & & & \\
\hline & -- & -- & -- & -- & -- & -- & -- & -- & -- \\
\hline & 10,373 & $\ldots$ & -- & -- & -- & -- & -. & -- & 2,485 \\
\hline & 5,696 & -- & -- & -- & -- & 5,696 & -- & -- & -- \\
\hline & 16,069 & $-\cdots$ & -- & -- & -- & 5,696 & -- & -- & 2,485 \\
\hline \multicolumn{10}{|l|}{$0 a k-g$ um } \\
\hline Sawt imber & 224,884 & -- & -- & -- & 1,184 & -- & -- & -- & 7,456 \\
\hline Poletimber & 10,803 & -- & & & & & -- & -- & -- \\
\hline Sapling \& seedling & 8,576 & -- & 2,052 & 1,651 & 3,839 & 1,034 & -- & -- & -- \\
\hline All stands & 244,263 & -- & 2,052 & 1,651 & 5,023 & 1,034 & -- & -- & 7,456 \\
\hline
\end{tabular}


(Table 51 continued)

\begin{tabular}{|c|c|c|c|c|c|c|c|c|c|}
\hline \multirow{2}{*}{$\begin{array}{l}\text { Forest type and } \\
\text { stand-size class }\end{array}$} & \multirow{2}{*}{$\begin{array}{c}\text { All } 1 \\
\text { classes }\end{array}$} & \multicolumn{8}{|c|}{ Basal area class (square feet per acre) } \\
\hline & & $0-10$ & $11-20$ & $21-30$ & $31-40$ & $41-50$ & $51-60$ & $61-70$ & $71-80$ \\
\hline \multirow{2}{*}{$\begin{array}{l}\text { Lowl and oak } \\
\text { Sawt imber } \\
\text { Pol et imber } \\
\text { Sapl ing \& seedling } \\
\text { All stands }\end{array}$} & $\begin{array}{r}152,967 \\
3,681 \\
1,990\end{array}$ & $\begin{array}{l}-- \\
--\end{array}$ & $\begin{array}{l}-- \\
-- \\
--\end{array}$ & $\begin{array}{r}-- \\
-- \\
1,990\end{array}$ & $\begin{array}{l}-- \\
--\end{array}$ & $\begin{array}{r}4,312 \\
-- \\
--\end{array}$ & $\begin{array}{l}-- \\
-- \\
--\end{array}$ & $\begin{array}{r}8,390 \\
3,681 \\
--\end{array}$ & $\begin{array}{l}-- \\
-- \\
--\end{array}$ \\
\hline & 158,638 & -- & -- & 1,990 & -- & 4,312 & -- & 12,071 & -- \\
\hline \multirow{2}{*}{$\begin{array}{l}\text { Elm-ash-so ft-maple } \\
\text { Sawt imber } \\
\text { Pol et imber } \\
\text { Sapl ing \& seedling } \\
\text { All Stands } \\
\end{array}$} & $\begin{array}{r}2,705,465 \\
286,612 \\
121,189 \\
\end{array}$ & $\begin{array}{l}-- \\
-- \\
-\end{array}$ & $\begin{array}{r}-- \\
4,656 \\
\end{array}$ & $\begin{array}{r}21,965 \\
1,033 \\
6,918 \\
\end{array}$ & $\begin{array}{r}29,635 \\
9,631 \\
20,144 \\
\end{array}$ & $\begin{array}{l}54,018 \\
10,862 \\
26,512 \\
\end{array}$ & $\begin{array}{l}30,908 \\
13,981 \\
25,070 \\
\end{array}$ & $\begin{array}{r}116,132 \\
34,791 \\
9,630 \\
\end{array}$ & $\begin{array}{r}249,419 \\
52,264 \\
19,835 \\
\end{array}$ \\
\hline & $3,113,266$ & -- & 4,656 & 29,916 & 59,410 & 91,392 & 69,959 & 160,553 & 321,518 \\
\hline \multirow{2}{*}{$\begin{array}{l}\text { Cottonwood } \\
\text { Sawtimber } \\
\text { Poletimber } \\
\text { Sapl ing \& seedling } \\
\text { All stands }\end{array}$} & $\begin{array}{r}96,156 \\
4,391 \\
-\end{array}$ & $\begin{array}{l}-- \\
-- \\
--\end{array}$ & $\begin{array}{l}-- \\
-- \\
--\end{array}$ & $\begin{array}{l}-- \\
-- \\
--\end{array}$ & $\begin{array}{r}3,204 \\
-- \\
--\end{array}$ & $\begin{array}{r}11,075 \\
- \\
--\end{array}$ & 4, 391 & $\begin{array}{l}-- \\
-- \\
--\end{array}$ & $\begin{array}{r}13,538 \\
-- \\
-.\end{array}$ \\
\hline & 100,547 & -- & -- & -- & 3,204 & 11,075 & 4,391 & -- & 13,538 \\
\hline \multirow{2}{*}{$\begin{array}{l}\text { Maple-beech } \\
\text { Sawt imber } \\
\text { Pol et imber } \\
\text { Sapl ing \& seedling } \\
\text { All stands }\end{array}$} & $\begin{array}{r}4,248,799 \\
222,226 \\
196,140 \\
\end{array}$ & $\begin{array}{l}-- \\
-- \\
-\end{array}$ & $\begin{array}{r}-- \\
8,065 \\
8,\end{array}$ & $\begin{array}{r}5,852 \\
-- \\
1,661 \\
\end{array}$ & $\begin{array}{r}11,046 \\
1,047 \\
15,811 \\
\end{array}$ & $\begin{array}{r}74,196 \\
4,458 \\
41,888 \\
\end{array}$ & $\begin{array}{r}236,741 \\
20,382 \\
28,662 \\
\end{array}$ & $\begin{array}{r}189,959 \\
25,715 \\
57,585 \\
\end{array}$ & $\begin{array}{r}524,838 \\
28,795 \\
28,164 \\
\end{array}$ \\
\hline & $4,667,165$ & -- & 8,065 & 7,513 & 27,904 & 120,542 & 285,785 & 273,259 & 581,797 \\
\hline \multirow{2}{*}{$\begin{array}{l}\text { Cherry-ash-yellow-pop } \\
\text { Sawtimber } \\
\text { Poletimber } \\
\text { Sapl ing \& seedling } \\
\text { All stands }\end{array}$} & $\begin{array}{r}\mathrm{ar} \\
2,322,789 \\
185,051 \\
117,465 \\
\end{array}$ & $\begin{array}{r}5,023 \\
- \\
- \\
\end{array}$ & $\begin{array}{r}-- \\
1,175 \\
\end{array}$ & $\begin{array}{l}7,869 \\
1,484 \\
8,425 \\
\end{array}$ & $\begin{array}{r}2,724 \\
-\therefore \\
9,749 \\
\end{array}$ & $\begin{array}{r}29,308 \\
-- \\
27,253 \\
\end{array}$ & $\begin{array}{l}50,486 \\
14,822 \\
25,462 \\
\end{array}$ & $\begin{array}{r}118,999 \\
5,181 \\
4,322 \\
\end{array}$ & $\begin{array}{r}148,627 \\
32,640 \\
20,868 \\
\end{array}$ \\
\hline & $2,625,305$ & 5,023 & 1,175 & 17,778 & 12,473 & 56,561 & 90,770 & 128,502 & 202,135 \\
\hline Nonstocked & 13,541 & -- & $\ldots$ & 3,921 & -- & -- & 7,408 & - & -- \\
\hline $\begin{array}{l}\text { All types } \\
\text { Sawtimber } \\
\text { Poletimber } \\
\text { Sapl ing \& seedling } \\
\text { Nonstocked }\end{array}$ & $\begin{array}{r}17,142,523 \\
1,302,871 \\
765,272 \\
13,541\end{array}$ & $\begin{array}{r}5,023 \\
-- \\
769 \\
--\end{array}$ & $\begin{array}{r}1,063 \\
-- \\
28,563 \\
--\end{array}$ & $\begin{array}{r}37,712 \\
3,650 \\
44,331 \\
3,921 \\
\end{array}$ & $\begin{array}{r}55,811 \\
11,617 \\
71,222 \\
--\end{array}$ & $\begin{array}{r}248,237 \\
22,128 \\
133,840 \\
\ldots\end{array}$ & $\begin{array}{r}529,824 \\
94,674 \\
174,551 \\
7,408 \\
\end{array}$ & $\begin{array}{r}610,702 \\
143,779 \\
131,616 \\
--\end{array}$ & $\begin{array}{r}1,458,772 \\
220,138 \\
109,130 \\
--\end{array}$ \\
\hline All stands & $19,224,207$ & 5,792 & 29,626 & 89,614 & 138,650 & 404,205 & 806,457 & 886,097 & $1,788,040$ \\
\hline
\end{tabular}


(Table 51 continued)

\begin{tabular}{|c|c|c|c|c|c|c|}
\hline \multirow{2}{*}{$\begin{array}{l}\text { Forest type and } \\
\text { stand-size class }\end{array}$} & \multicolumn{6}{|c|}{ Basal area class (square feet per acre) } \\
\hline & $81-90$ & $91-100$ & $101-120$ & $121-150$ & $151-180$ & $181+$ \\
\hline $\begin{array}{l}\text { Jack-red-white pine } \\
\text { Sawtimber } \\
\text { Poletimber } \\
\text { Sapling \& seedling }\end{array}$ & $\begin{array}{r}17,110 \\
2,400 \\
--\end{array}$ & 2,971 & $\begin{array}{r}46,382 \\
18,668\end{array}$ & 7,764 & $\begin{array}{r}74,001 \\
10,261\end{array}$ & $\begin{array}{l}-- \\
--\end{array}$ \\
\hline Al 1 stands & 19,510 & 2,971 & 65,050 & 7,764 & 84,262 & -- \\
\hline $\begin{array}{l}\text { Shortleaf pine } \\
\text { Sawtimber } \\
\text { Poletimber } \\
\text { Sapling \& seedling }\end{array}$ & $\begin{array}{r}9,869 \\
-- \\
-- \\
\end{array}$ & $\begin{array}{l}-- \\
-- \\
-\end{array}$ & $\begin{array}{r}22,232 \\
46,131 \\
--\end{array}$ & $\begin{array}{r}11,684 \\
-- \\
- \\
\end{array}$ & $\begin{array}{l}-- \\
-- \\
--\end{array}$ & -- \\
\hline Al 1 stands & 9,869 & -- & 68,363 & 11,684 & -- & - \\
\hline $\begin{array}{l}\text { Scotch-Virginia pine } \\
\text { Sawtimber } \\
\text { Poletimber } \\
\text { Sapling \& seedling }\end{array}$ & $\begin{array}{r}32,554 \\
579 \\
-- \\
\end{array}$ & $\begin{array}{r}=- \\
10,139 \\
-=\end{array}$ & $\begin{array}{r}59,383 \\
4,960 \\
898 \\
\end{array}$ & $\begin{array}{r}46,964 \\
-- \\
=- \\
\end{array}$ & $\begin{array}{r}=- \\
29,325 \\
--\end{array}$ & $\begin{array}{l}-- \\
-- \\
-\end{array}$ \\
\hline A1 1 stands & 33,133 & 10,139 & 65,241 & 46,964 & 29,325 & -- \\
\hline $\begin{array}{l}\text { Oak-pine } \\
\text { Sawtimber } \\
\text { Poletimber } \\
\text { Sapling \& seedling }\end{array}$ & $\begin{array}{r}39,236 \\
5,020 \\
1,961 \\
\end{array}$ & $\begin{array}{r}7,686 \\
- \\
-- \\
\end{array}$ & $\begin{array}{r}112,430 \\
7,711 \\
\cdots\end{array}$ & $\begin{array}{r}30,556 \\
15,172 \\
-- \\
\end{array}$ & $\begin{array}{l}-- \\
-- \\
--\end{array}$ & $\begin{array}{l}-- \\
-- \\
-\end{array}$ \\
\hline A11 stands & 46,217 & 7,686 & 120,141 & 45,728 & -- & -- \\
\hline $\begin{array}{l}\text { Oak-hickory } \\
\text { Sawt imber } \\
\text { Poletimber } \\
\text { Sapling \& seedling }\end{array}$ & $\begin{array}{r}1,255,399 \\
57,498 \\
24,590 \\
\end{array}$ & $\begin{array}{r}921,227 \\
59,329 \\
\ldots\end{array}$ & $\begin{array}{r}2,229,496 \\
53,232 \\
862 \\
\end{array}$ & $\begin{array}{r}1,018,708 \\
20,231 \\
-- \\
\end{array}$ & $\begin{array}{r}108,927 \\
-- \\
-- \\
\end{array}$ & $\begin{array}{r}14,066 \\
-- \\
=-\end{array}$ \\
\hline Al 1 stands & $1,337,487$ & 980,556 & $2,283,590$ & $1,038,939$ & 108,927 & 14,066 \\
\hline $\begin{array}{l}\text { Chestnut-scarlet oak } \\
\text { Sawtimber } \\
\text { Poletimber } \\
\text { Sapl ing \& seedling }\end{array}$ & $\begin{array}{r}91,179 \\
-- \\
-- \\
\end{array}$ & $\begin{array}{r}62,472 \\
-- \\
-- \\
\end{array}$ & $\begin{array}{r}142,576 \\
\ldots \\
\ldots \\
\end{array}$ & $\begin{array}{r}47,086 \\
-- \\
-- \\
\end{array}$ & $\begin{array}{l}-- \\
-- \\
-\end{array}$ & $\begin{array}{l}-- \\
-- \\
-\end{array}$ \\
\hline All stands & 91,179 & 62,472 & 142,576 & 47,086 & -- & $\cdots$ \\
\hline $\begin{array}{l}\text { Sassa fras-persimmon } \\
\text { Sawt imber } \\
\text { Polet imber } \\
\text { Sapling \& seedling }\end{array}$ & $\begin{array}{l}-- \\
-- \\
--\end{array}$ & $\begin{array}{l}-- \\
-- \\
-\end{array}$ & $\begin{array}{r}-- \\
7,888 \\
-- \\
\end{array}$ & $\begin{array}{l}-- \\
-- \\
\end{array}$ & $\begin{array}{l}-- \\
-- \\
-\end{array}$ & $\begin{array}{l}-- \\
-- \\
-\end{array}$ \\
\hline All stands & -- & -- & 7,888 & -- & - & -- \\
\hline $\begin{array}{l}\text { Oak-gum } \\
\text { Sawtimber } \\
\text { Poletimber } \\
\text { Sapling \& seedling }\end{array}$ & $7,1 \overline{1}$ & $\begin{array}{r}51,562 \\
-- \\
-- \\
\end{array}$ & $\begin{array}{r}98,452 \\
3,662 \\
-- \\
\end{array}$ & $\begin{array}{r}66,230 \\
-- \\
--\end{array}$ & $\begin{array}{l}-- \\
-- \\
--\end{array}$ & $\begin{array}{l}-- \\
-- \\
-\end{array}$ \\
\hline Al 1 stands & 7,141 & 51,562 & 102,114 & 66,230 & -- & -- \\
\hline
\end{tabular}


(Table 51 continued)

\begin{tabular}{|c|c|c|c|c|c|c|}
\hline \multirow{2}{*}{$\begin{array}{l}\text { Forest type and } \\
\text { stand-size class }\end{array}$} & \multicolumn{6}{|c|}{ Basal area class (square feet per acre) } \\
\hline & $81-90$ & $91-100$ & $101-120$ & $121-150$ & $151-180$ & $181+$ \\
\hline $\begin{array}{l}\text { Lowl and oak } \\
\text { Sawtimber } \\
\text { Poletimber } \\
\text { Sapling \& seedling }\end{array}$ & $\begin{array}{r}18,565 \\
-- \\
-- \\
\end{array}$ & $\begin{array}{l}-- \\
-- \\
--\end{array}$ & $\begin{array}{r}121,700 \\
--- \\
-- \\
\end{array}$ & $\begin{array}{l}-- \\
-- \\
--\end{array}$ & -- & $\begin{array}{l}-- \\
--\end{array}$ \\
\hline All stands & 18,565 & -- & 121,700 & -- & - & -- \\
\hline $\begin{array}{l}\text { Elm-ash-soft-maple } \\
\text { Sawtimber } \\
\text { Poletimber } \\
\text { Sapling \& seedling }\end{array}$ & $\begin{array}{r}338,791 \\
60,704 \\
8,424 \\
\end{array}$ & $\begin{array}{r}390,027 \\
29,572 \\
- \\
\end{array}$ & $\begin{array}{r}721,452 \\
73,774 \\
-- \\
\end{array}$ & $\begin{array}{r}513,363 \\
-- \\
-- \\
\end{array}$ & $\begin{array}{r}190,648 \\
-- \\
--\end{array}$ & $\begin{array}{r}49,107 \\
\ldots \\
-\end{array}$ \\
\hline A1 1 stands & 407,919 & 419,599 & 795,226 & 513,363 & 190,648 & 49,107 \\
\hline $\begin{array}{l}\text { Cottonwood } \\
\text { Sawtimber } \\
\text { Poletimber } \\
\text { Sapling \& seedling }\end{array}$ & $\begin{array}{l}-- \\
-- \\
--\end{array}$ & $\begin{array}{l}-- \\
-- \\
-\end{array}$ & $\begin{array}{r}33,703 \\
-- \\
-- \\
\end{array}$ & $\begin{array}{r}34,636 \\
-- \\
-- \\
\end{array}$ & $\begin{array}{l}-- \\
-- \\
--\end{array}$ & $\begin{array}{l}- \\
-- \\
--\end{array}$ \\
\hline All stands & -- & -- & 33,703 & 34,636 & -- & $\cdots$ \\
\hline $\begin{array}{l}\text { Maple-beech } \\
\text { Sawtimber } \\
\text { Poletimber } \\
\text { Sapling \& seedling }\end{array}$ & $\begin{array}{r}614,695 \\
34,471 \\
--- \\
\end{array}$ & $\begin{array}{r}519,970 \\
9,892 \\
2,522 \\
\end{array}$ & $\begin{array}{r}1,320,443 \\
85,612 \\
11,782 \\
\end{array}$ & $\begin{array}{r}630,264 \\
11,854 \\
-- \\
\end{array}$ & $\begin{array}{r}120,795 \\
-- \\
-\end{array}$ & $\begin{array}{l}-: \\
--\end{array}$ \\
\hline Al1 stands & 649,166 & 532,384 & $1,417,837$ & 642,118 & 120,795 & -- \\
\hline $\begin{array}{l}\text { Cherry-ash-yellow-pop } \\
\text { Sawtimber } \\
\text { Poletimber } \\
\text { Sapling \& seedling }\end{array}$ & $\begin{array}{r}294,233 \\
57,380 \\
14,100 \\
\end{array}$ & $\begin{array}{r}221,293 \\
16,702 \\
6,111 \\
\end{array}$ & $\begin{array}{r}902,754 \\
28,337 \\
-- \\
\end{array}$ & $\begin{array}{r}452,755 \\
28,505 \\
-- \\
\end{array}$ & $\begin{array}{r}52,466 \\
-- \\
-- \\
\end{array}$ & $\begin{array}{r}36,252 \\
-- \\
-- \\
\end{array}$ \\
\hline All stands & 365,713 & 244,106 & 931,091 & 481,260 & 52,466 & 36,252 \\
\hline Nons tocked & -- & 2,212 & -- & -- & -- & -- \\
\hline $\begin{array}{l}\text { All types } \\
\text { Sawtimber } \\
\text { Poletimber } \\
\text { Sapl ing \& seedl ing } \\
\text { Nonstocked }\end{array}$ & $\begin{array}{r}2,711,631 \\
225,193 \\
49,075 \\
--\end{array}$ & $\begin{array}{r}2,174,237 \\
128,605 \\
8,633 \\
2,212\end{array}$ & $\begin{array}{r}5,811,003 \\
329,975 \\
13,542 \\
--\end{array}$ & $\begin{array}{r}2,852,246 \\
83,526 \\
-- \\
--\end{array}$ & $\begin{array}{r}546,837 \\
39,586 \\
-- \\
--\end{array}$ & $\begin{array}{r}99,425 \\
-- \\
-- \\
--\end{array}$ \\
\hline All stands & $2,985,899$ & $2,313,687$ & $6,154,520$ & $2,935,772$ & 586,423 & 99,425 \\
\hline
\end{tabular}


Table 52.--Net volume of growing stock and sawtimber on timberland by county and species group, Indiana, 1986

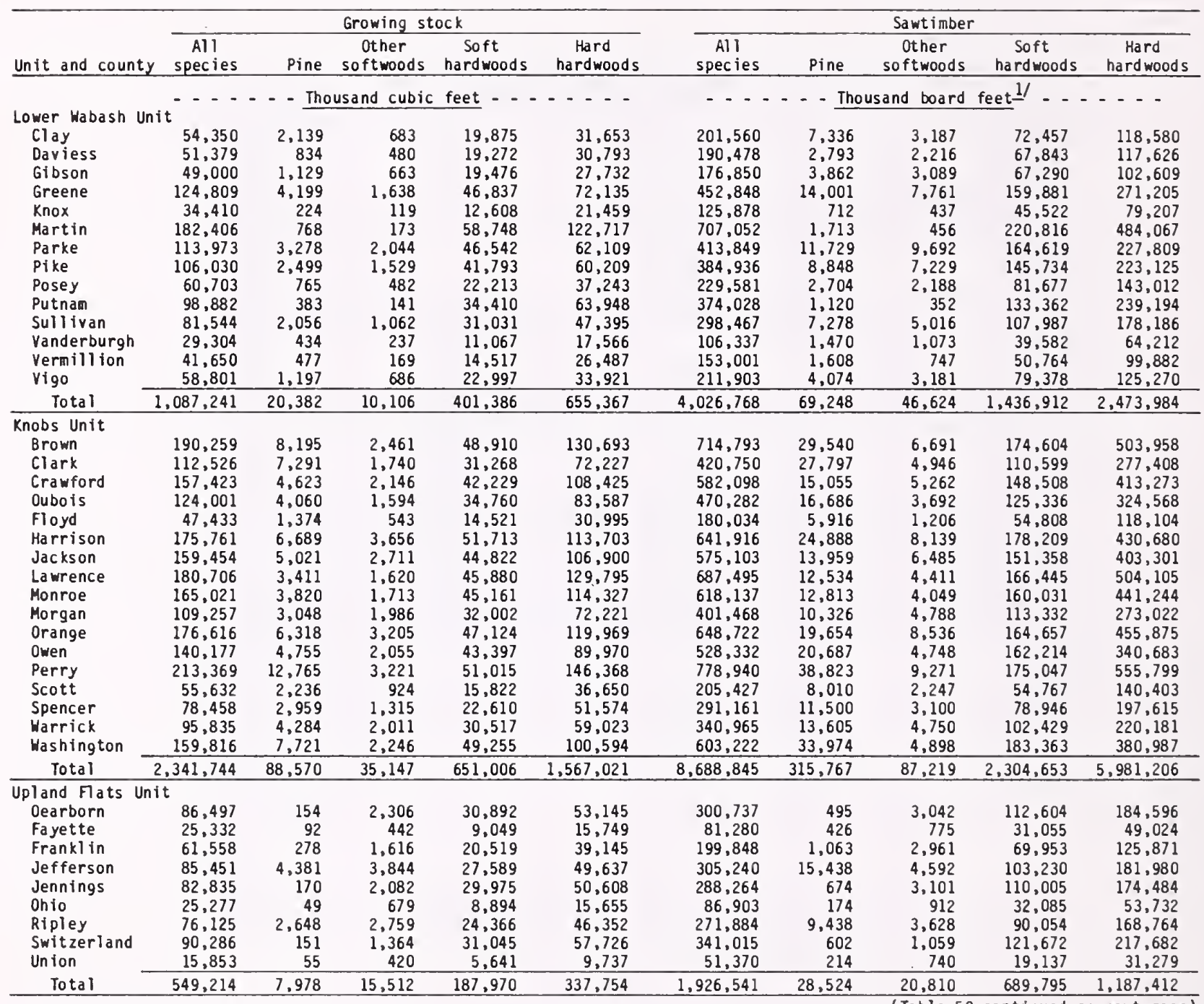

1/International $1 / 4$-inch rule. 
(Table 52 continued)

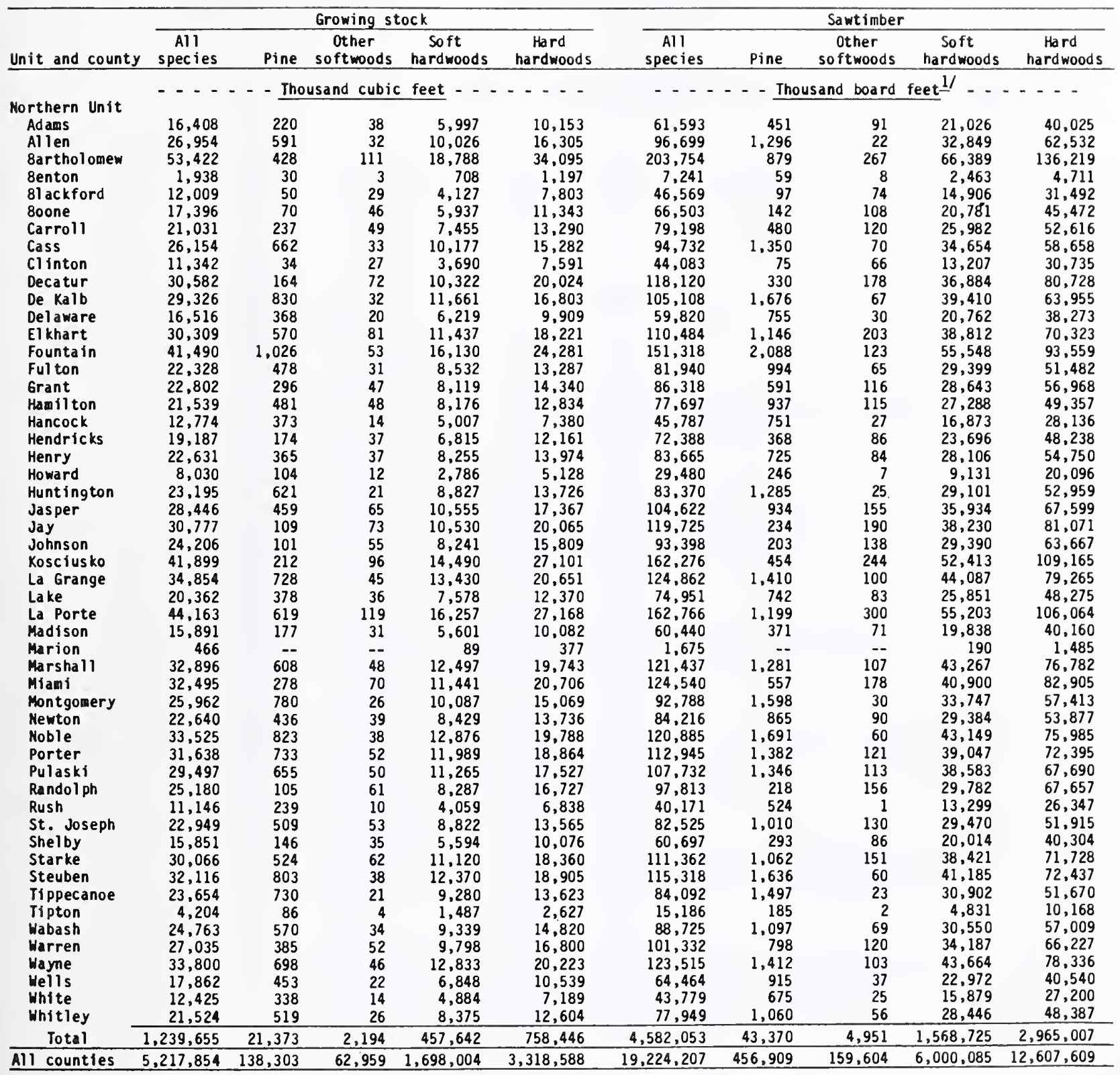

1/International 1/4-inch rule. 
Table 53.--Net volume of sawtimber on timberland by species group and tree grade, Indiana, 1986 (In thousand board feet) 1 /

\begin{tabular}{|c|c|c|c|c|c|}
\hline \multirow[b]{2}{*}{ Species group } & \multirow{2}{*}{$\begin{array}{c}\text { All } \\
\text { species }\end{array}$} & \multicolumn{4}{|c|}{ Tree grade } \\
\hline & & 1 & 2 & 3 & Tie and timber \\
\hline $\begin{array}{l}\text { Softwoods } \\
\text { Jack pine } \\
\text { Red pine } \\
\text { White pine } \\
\text { Shortleaf pine } \\
\text { Other yellow pines } \\
\text { Tamarack } \\
\text { 8aldcypress } \\
\text { Eastern redcedar } \\
\text { Other softwoods }\end{array}$ & $\begin{array}{r}18,409 \\
11,919 \\
151,387 \\
87,129 \\
183,341 \\
4,208 \\
41,635 \\
113,761 \\
4,724\end{array}$ & $\begin{array}{r}-- \\
-- \\
6,862 \\
-- \\
-- \\
-- \\
-- \\
-- \\
--\end{array}$ & $\begin{array}{r}- \\
-- \\
1,475 \\
7,837 \\
6,658 \\
-- \\
2,098 \\
2,177 \\
\ldots\end{array}$ & $\begin{array}{r}18,409 \\
11,919 \\
126,306 \\
79,292 \\
175,749 \\
4,208 \\
39,537 \\
110,712 \\
4,724 \\
\end{array}$ & $\begin{array}{r}\ldots- \\
16,744 \\
-- \\
934 \\
-- \\
-- \\
872 \\
-.\end{array}$ \\
\hline Total & 616,513 & 6,862 & 20,245 & 570,856 & 18,550 \\
\hline $\begin{array}{l}\text { Hardwoods } \\
\text { Select white oak } \\
\text { Other white oak } \\
\text { Select red oak } \\
\text { Other red oak } \\
\text { Select hickory } \\
\text { Other hickory } \\
\text { 8asswood } \\
\text { 8eech } \\
\text { Hard maple } \\
\text { Soft maple } \\
\text { Elm } \\
\text { Ash } \\
\text { Sycamore } \\
\text { Cottonwood } \\
\text { Willow } \\
\text { Hackberry } \\
\text { Aspen } \\
\text { 8irch } \\
\text { Sweetgum } \\
\text { Tupelo } \\
\text { 8lack cherry } \\
\text { 8lack walnut } \\
\text { 8utternut } \\
\text { Yellow-poplar } \\
\text { Persimmon } \\
\text { Sassafras } \\
\text { Other hardwoods }\end{array}$ & $\begin{array}{r}2,875,793 \\
533,134 \\
1,316,182 \\
2,163,418 \\
939,742 \\
1,003,171 \\
253,100 \\
608,945 \\
1,413,239 \\
809,358 \\
329,362 \\
1,174,946 \\
920,914 \\
587,853 \\
45,497 \\
171,147 \\
110,582 \\
31,507 \\
222,029 \\
155,667 \\
318,703 \\
427,405 \\
15,368 \\
1,840,013 \\
10,011 \\
162,154 \\
168,454\end{array}$ & $\begin{array}{r}457,221 \\
15,331 \\
89,266 \\
110,707 \\
31,885 \\
46,503 \\
35,682 \\
-- \\
39,780 \\
5,742 \\
20,241 \\
155,788 \\
203,738 \\
74,377 \\
2,635 \\
11,996 \\
-- \\
2,827 \\
14,347 \\
11,453 \\
17,220 \\
46,038 \\
-- \\
191,596 \\
-- \\
5,558 \\
1,083\end{array}$ & $\begin{array}{r}781,401 \\
99,798 \\
187,078 \\
260,533 \\
99,488 \\
145,824 \\
71,475 \\
4,637 \\
134,478 \\
68,788 \\
70,656 \\
336,442 \\
256,111 \\
164,863 \\
3,946 \\
53,025 \\
5,409 \\
3,504 \\
48,417 \\
31,317 \\
60,998 \\
126,987 \\
3,658 \\
216,972 \\
1,909 \\
30,387 \\
16,323 \\
\end{array}$ & $\begin{array}{r}1,016,708 \\
229,002 \\
387,335 \\
569,454 \\
310,018 \\
375,600 \\
114,921 \\
48,263 \\
488,919 \\
203,212 \\
156,033 \\
491,615 \\
320,924 \\
181,615 \\
11,781 \\
74,387 \\
20,879 \\
6,849 \\
79,708 \\
42,258 \\
122,139 \\
214,268 \\
9,197 \\
391,441 \\
8,102 \\
90,469 \\
54,608 \\
\end{array}$ & $\begin{array}{r}620,463 \\
189,003 \\
652,503 \\
1,222,724 \\
498,351 \\
435,244 \\
31,022 \\
556,045 \\
750,062 \\
531,616 \\
82,432 \\
191,101 \\
140,141 \\
166,998 \\
27,135 \\
31,739 \\
84,294 \\
18,327 \\
79,557 \\
70,639 \\
118,346 \\
40,112 \\
2,513 \\
1,040,004 \\
35,740 \\
96,440 \\
\end{array}$ \\
\hline Total & $18,607,694$ & $1,591,014$ & $3,284,424$ & $6,019,705$ & $7,712,551$ \\
\hline All species & $19,224,207$ & $1,597,876$ & $3,304,669$ & $6,590,561$ & $7,731,101$ \\
\hline
\end{tabular}

$1 /$ International $1 / 4$-inch rule. 


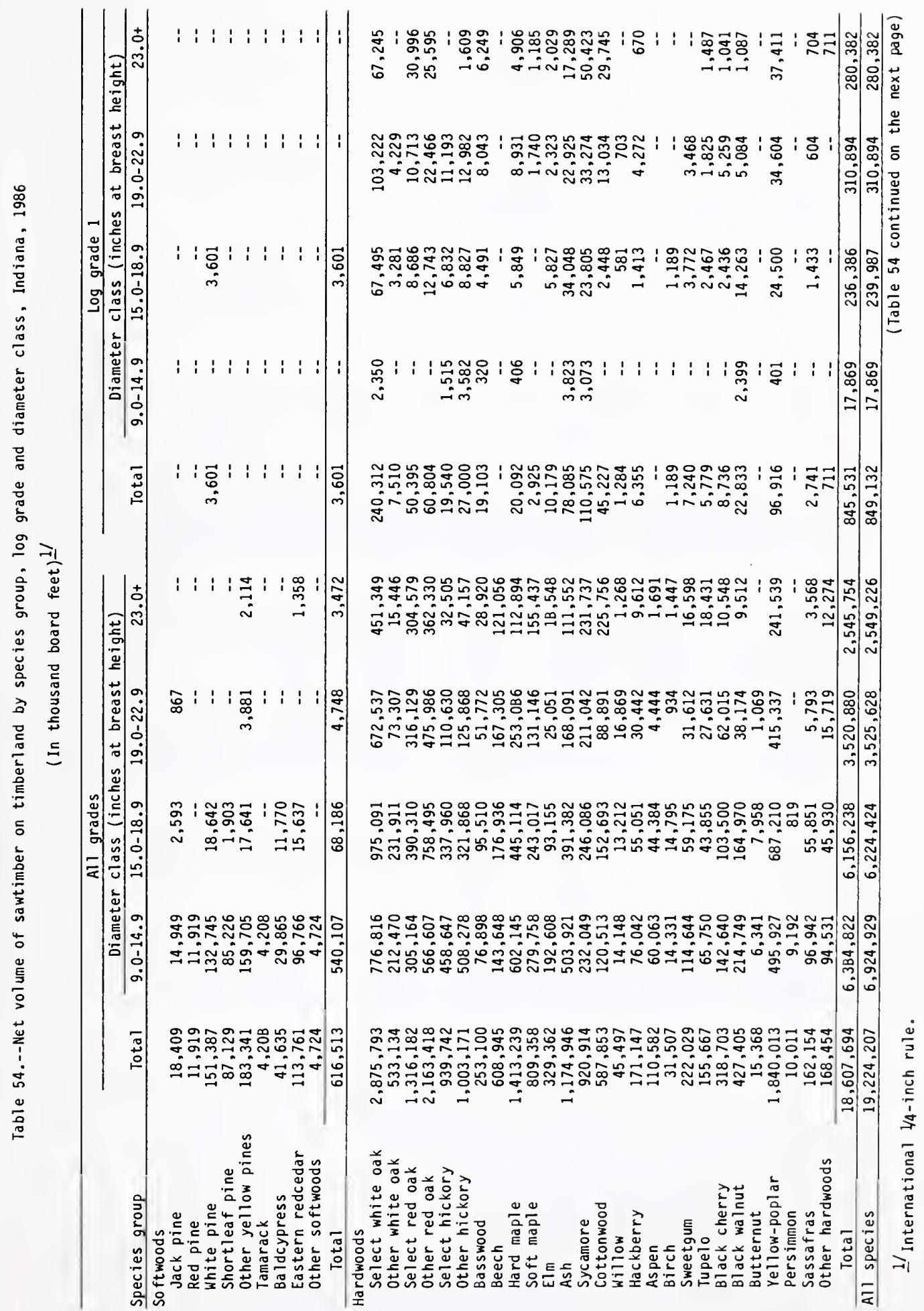




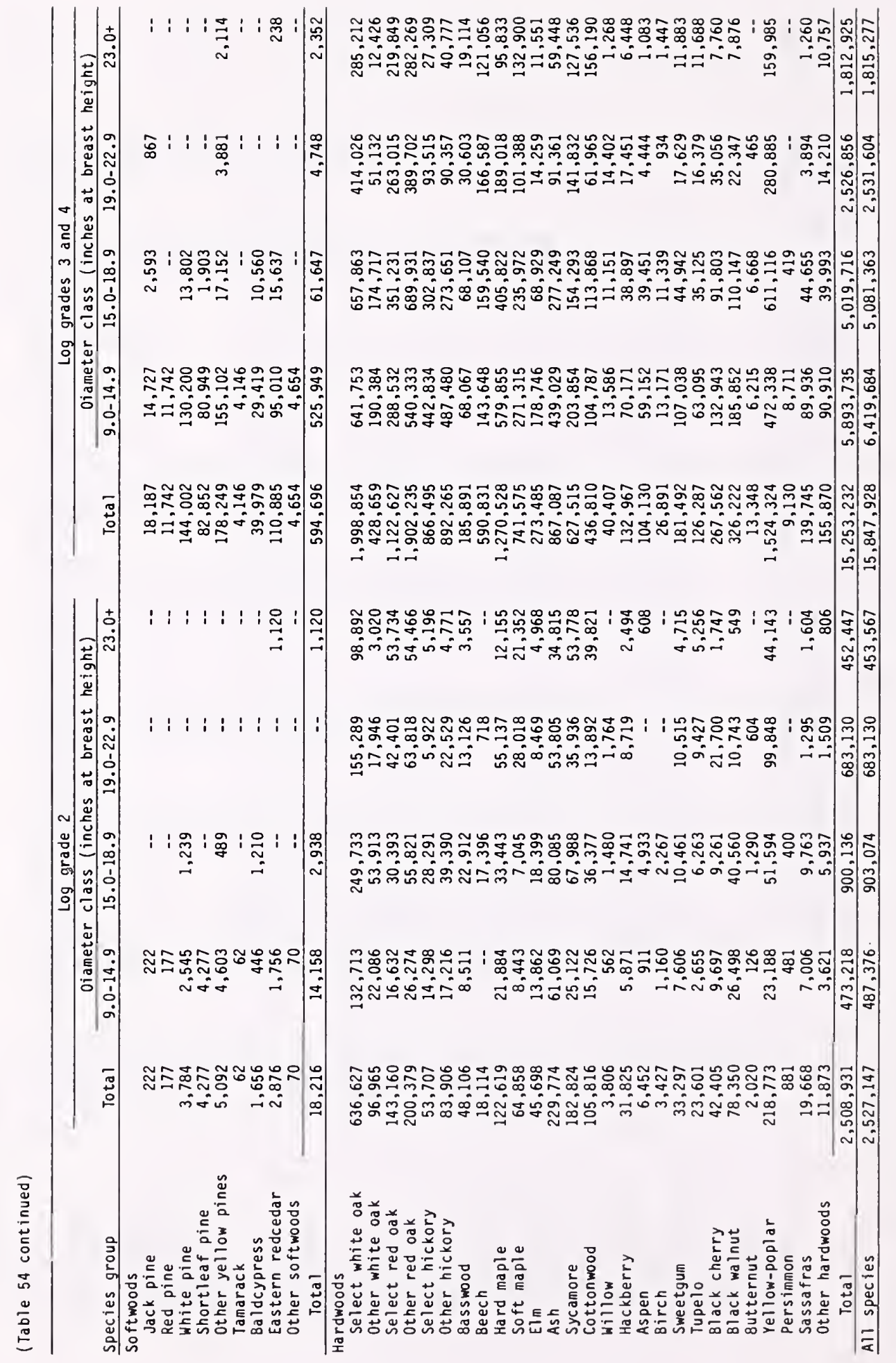


Jable 55.-- Net volume of short-log trees on timberland by species group and diameter class, Indiana, 1986

(In thousand cubic feet)

\begin{tabular}{|c|c|c|c|c|c|c|c|c|c|c|c|}
\hline \multirow[b]{2}{*}{ Species group } & \multirow[b]{2}{*}{$\begin{array}{c}\text { All } \\
\text { classes }\end{array}$} & \multicolumn{10}{|c|}{ 0iameter class (inches at breast height) } \\
\hline & & $\begin{array}{l}9.0- \\
10.9\end{array}$ & $\begin{array}{l}11.0= \\
12.9\end{array}$ & $\begin{array}{l}13.0- \\
14.9\end{array}$ & $\begin{array}{l}15.0- \\
16.9\end{array}$ & $\begin{array}{l}17.0- \\
18.9\end{array}$ & $\begin{array}{l}19.0- \\
20.9\end{array}$ & $\begin{array}{l}21.0- \\
22.9\end{array}$ & $\begin{array}{l}23.0- \\
28.9\end{array}$ & $\begin{array}{l}29.0- \\
38.9\end{array}$ & $39.0+$ \\
\hline \multicolumn{12}{|l|}{ So ftwoods } \\
\hline Jack pine & 199 & 199 & - & -. & -- & -- & - & -- & - & -- & $\cdots$ \\
\hline Red pine & -- & - & -- & -- & -- & -- &.- & -- & -- & -- & -- \\
\hline White pine & -- & -- & -- & -- & -- & -- & -- & -- & -- & -- & -- \\
\hline Shortleaf pine & 488 & 130 & 358 & -- & -- & - & $\cdots$ & -- & -- & -- & - \\
\hline other yellow pines & 236 & 104 & - & -- & -- & - & -- & 132 & -- & -- & - \\
\hline Tamarack & - & - & -- & -- & -- & - & -- & - & -- & -- & - \\
\hline Baldcypress & -- & -- & -- & -- & -- & -- & -. & - & -- & -- & -. \\
\hline Eastern redcedar & 885 & 465 & 130 & 140 & -- & 150 & -- & -- & -- & -- & -- \\
\hline other softwoods & -- & - & -- & - & - & -- & -- & - & -- & - & -- \\
\hline Total & 1,808 & 898 & 488 & 140 & -- & 150 & -- & 132 & -- & -- & - \\
\hline \multicolumn{12}{|l|}{ Hardwoods } \\
\hline Select white oak & 21,026 & - & 2,284 & 2,290 & 1,501 & 2,443 & 1,279 & 2,397 & 4,289 & 3,177 & 1,366 \\
\hline other white oak & 1,875 & -- & 359 & 103 & 829 & 128 & 170 & $=$ & 286 & & \\
\hline Select red oak & 9,521 & - & - & 504 & 633 & 1,096 & 1,443 & 620 & 1,731 & 2,058 & 1,436 \\
\hline other red oak & 11,533 & -- & 1,113 & 170 & 1,161 & 1,601 & 2,039 & 1,397 & 1,400 & 1,488 & 1,164 \\
\hline Select hickory & 6,442 & -- & 631 & 1,651 & 1,341 & 1,268 & 828 & 395 & 328 & - & -- \\
\hline Other hickory & 5,889 & -- & 1,438 & 1,053 & 906 & 335 & 291 & 731 & 1,135 & - & -- \\
\hline 8asswood & 2,287 & -- & 256 & 122 & 155 & 616 & 134 & - & 749 & 255 & -- \\
\hline Beech & 8,899 & -- & 138 & 1,512 & 879 & 1,781 & 1,548 & 748 & 1,183 & 951 & 159 \\
\hline Hard maple & 19,052 & -- & 3,115 & 3,260 & 3,028 & 1,439 & 1,600 & 2,013 & 3,570 & 1,027 & -- \\
\hline Soft maple & 15,028 & -- & 2,654 & 1,258 & 1,493 & 2,208 & 1,696 & 1,150 & 2,719 & 1,230 & 620 \\
\hline Elm & 4,739 & -- & 1,419 & 1,322 & 539 & 648 & 242 & 214 & 241 & 114 & - \\
\hline Ash & 14,414 & -- & 3,442 & 1,974 & 2,207 & 2,244 & 1,475 & 888 & 1,063 & 814 & 307 \\
\hline Sycamore & 4,151 & - & 137 & 1,214 & 805 & 134 & 798 & - & 511 & 472 & 80 \\
\hline Cottonwood & 4,127 & - & 286 & 574 & - & -- & 1,010 & -. & 528 & 1,373 & 356 \\
\hline Willow & 1,589 & -- & 238 & 230 & 501 & -- & - & - & 393 & 227 & - \\
\hline Hackberry & 2,633 & -- & 444 & 478 & 373 & 128 & 154 & 132 & 426 & -- & 498 \\
\hline Aspen & 109 & -- & 109 & - & -- & - & -- & -- & -- & -- & -- \\
\hline Birch & 170 & -- & -- & -- & -- & - & -- & -- & 170 & -- & -- \\
\hline Sweetgum & 586 & -- & -- & -- & 147 & 232 & -- & -- & 129 & 78 & -- \\
\hline Tupelo & 1,102 & -- & 348 & 316 & 339 & -- & -- & -- & 99 & -- & -- \\
\hline Black cherry & 6,192 & -- & 2,229 & 798 & 752 & 837 & -- & 758 & 518 & 300 & -- \\
\hline Black walnut & 6,385 & -- & 1,968 & 1,609 & 689 & 579 & 276 & 399 & 587 & 158 & 120 \\
\hline Butternut & 298 & -- & 298 & - & - & - & -- & - & -- & -- & - \\
\hline Yel low-poplar & 3,874 & -- & 1,030 & 693 & 124 & 817 & 46 & 56 & 698 & 260 & 150 \\
\hline Persimmon & - & -- & - & - & - & - & - & - & -- & - & -- \\
\hline Sassafras & 3,101 & -- & 565 & 313 & 592 & 589 & 297 & - & 513 & 232 & -- \\
\hline Other hardwoods & 5,438 & -- & 1,500 & 1,657 & 874 & 624 & 596 & -- & 187 & $\cdots$ & $=$ \\
\hline Total & 160,460 & -- & 26,001 & 23,101 & 19,868 & 19,747 & 15,922 & 11,898 & 23,453 & 14,214 & 6,256 \\
\hline All species & 162.268 & 898 & 26,489 & 23,241 & 19,868 & 19,897 & 15,922 & 12,030 & 23,453 & 14,214 & 6,256 \\
\hline
\end{tabular}


Table 56.--Net volume of short-log trees on timberland by species group and diameter class, Indiana, 1986 (In thousand board feet) 1 '

\begin{tabular}{|c|c|c|c|c|c|c|c|c|c|c|c|}
\hline \multirow[b]{2}{*}{ Spectes group } & \multirow[b]{2}{*}{$\begin{array}{c}\text { All } \\
\text { classes }\end{array}$} & \multicolumn{10}{|c|}{ Diameter class (inches at breast height) } \\
\hline & & $\begin{array}{r}9.0- \\
10.9 \\
\end{array}$ & $\begin{array}{l}11.0- \\
12.9 \\
\end{array}$ & $\begin{array}{l}13.0- \\
14.9 \\
\end{array}$ & $\begin{array}{l}15.0- \\
16.9 \\
\end{array}$ & $\begin{array}{l}17.0- \\
18.9\end{array}$ & $\begin{array}{l}19.0- \\
20.9 \\
\end{array}$ & $\begin{array}{l}21.0- \\
22.9 \\
\end{array}$ & $\begin{array}{l}23.0- \\
28.9 \\
\end{array}$ & $\begin{array}{l}29.0- \\
38.9 \\
\end{array}$ & $39.0+$ \\
\hline \multicolumn{12}{|l|}{$\begin{array}{l}\text { So ft woods } \\
\text { Jack pine }\end{array}$} \\
\hline $\begin{array}{l}\text { Jack pine } \\
\text { Red pine }\end{array}$ & $\begin{array}{r}1,008 \\
-.\end{array}$ & $\begin{array}{r}1,008 \\
\ldots\end{array}$ & $\begin{array}{l}-- \\
--\end{array}$ & -- & -- & -- & -- & -- & - & -. & -- \\
\hline White pine & - & - & -- & 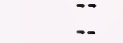 & $\begin{array}{l}-- \\
--\end{array}$ & $\begin{array}{l}-- \\
--\end{array}$ & -- & -- & -- & -- & -- \\
\hline Shortleaf pine & 2,254 & 654 & 1,600 & -. & -- & -- & -- & $\because$ & -- & $-\cdot$ & -- \\
\hline Other yellow pines & 887 & 525 & -- & -- & -- & - & -- & 362 & -- & -- & -- \\
\hline Tamarack & -- & -- & -- & -- & -- & -- & -- & -- & -- & - & $\begin{array}{l}-- \\
--\end{array}$ \\
\hline Baldcypress & -- & -- & -- & -- & -- & -- & -- & -- & -- & -. & $\therefore$ \\
\hline Eastern redcedar & 3,990 & 2,353 & $\begin{array}{r}581 \\
--\end{array}$ & 557 & -- & 499 & -- & -- & -- & -- & -- \\
\hline $\begin{array}{l}\text { Uther softwoods } \\
\text { Total }\end{array}$ & -- & -- & $\frac{--}{2,181}$ & -- & -- & -- & $\because$ & 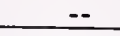 & - & $\cdots$ & -- \\
\hline \multicolumn{8}{|l|}{ Hardwoods } & 362 & & & \\
\hline $\begin{array}{l}\text { Select white oak } \\
\text { other white oak }\end{array}$ & $\begin{array}{r}63,145 \\
6,111\end{array}$ & $\begin{array}{l}-- \\
--\end{array}$ & $\begin{array}{l}8,474 \\
1,332\end{array}$ & $\begin{array}{r}7,991 \\
358\end{array}$ & $\begin{array}{l}4,968 \\
2,745\end{array}$ & $\begin{array}{r}7,567 \\
397\end{array}$ & $\begin{array}{r}3,924 \\
522\end{array}$ & $\begin{array}{r}6,814 \\
-.\end{array}$ & 11,365 & 8,419 & 3,623 \\
\hline Select red oak & 27,278 & -- & - & 1,760 & 2,095 & 3,391 & 4,423 & 1,763 & 4,583 & 5,454 & $3,8 \overline{-}$ \\
\hline $\begin{array}{l}\text { Other red oak } \\
\text { Select hickory }\end{array}$ & $\begin{array}{l}34,482 \\
21,000\end{array}$ & -- & $\begin{array}{l}4,132 \\
2,341\end{array}$ & $\begin{array}{r}594 \\
5,766\end{array}$ & $\begin{array}{l}3,847 \\
4,442\end{array}$ & $\begin{array}{l}4,960 \\
3,925\end{array}$ & $\begin{array}{l}6,248 \\
2,534\end{array}$ & $\begin{array}{l}3,968 \\
1,123\end{array}$ & $\begin{array}{r}3,704 \\
869\end{array}$ & 3,945 & 3,084 \\
\hline Other hickory & 19,030 & - & 5,342 & 3,679 & 2,997 & 1,037 & 893 & 2,076 & $\begin{array}{r}869 \\
3,006\end{array}$ & $\because$ & $\begin{array}{l}-- \\
--\end{array}$ \\
\hline $\begin{array}{l}\text { Basswood } \\
\text { Beech }\end{array}$ & $\begin{array}{r}6,873 \\
27,168\end{array}$ & -- & 949 & 428 & 515 & 1,909 & 411 & -. & 1,986 & 675 & -- \\
\hline Hard maple & 60,238 & -- & $\begin{array}{r}512 \\
11,568\end{array}$ & $\begin{array}{r}5,281 \\
11,385\end{array}$ & $\begin{array}{r}2,910 \\
10,019\end{array}$ & $\begin{array}{l}5,521 \\
4,456\end{array}$ & $\begin{array}{l}4,747 \\
4,905\end{array}$ & $\begin{array}{l}2,125 \\
5,721\end{array}$ & $\begin{array}{l}3,133 \\
9,460\end{array}$ & 2,519 & 420 \\
\hline $\begin{array}{l}\text { So ft maple } \\
\text { Elm }\end{array}$ & $\begin{array}{l}46,581 \\
15,954\end{array}$ & $\begin{array}{ll}-- \\
--\end{array}$ & $\begin{array}{l}9,847 \\
5,264\end{array}$ & $\begin{array}{l}4,390 \\
4,614\end{array}$ & $\begin{array}{l}4,944 \\
1,785\end{array}$ & $\begin{array}{l}6,836 \\
2,005\end{array}$ & $\begin{array}{r}5,198 \\
740\end{array}$ & $\begin{array}{r}3,268 \\
607\end{array}$ & 7,200 & $\begin{array}{l}2,724 \\
3,260\end{array}$ & 1,638 \\
\hline Ash & 46,751 & -- & 12,776 & 6,897 & 7,301 & 6,946 & 4,523 & 2,524 & 2,817 & $\begin{array}{r}300 \\
2,157\end{array}$ & $8 \overline{10}$ \\
\hline Sycamore & 13,092 & -- & 508 & 4,240 & 2,666 & 415 & 2,446 & -- & 1,354 & 1,252 & $\begin{array}{l}810 \\
211\end{array}$ \\
\hline Cottonwood & 12,138 & -- & 1,061 & 2,003 & -- & -- & 3,096 & -- & 1,399 & 3,637 & 942 \\
\hline Willow & 4,985 & $\cdots$ & 881 & 803 & 1,658 & -- & -- & -- & 1,042 & 601 & -- \\
\hline $\begin{array}{l}\text { Hackberry } \\
\text { Aspen }\end{array}$ & & -- & 1,651 & 1,667 & 1,235 & 396 & 472 & 376 & 1,130 & - & 1,317 \\
\hline $\begin{array}{l}\text { Aspen } \\
\text { Birch }\end{array}$ & $\begin{array}{l}405 \\
451\end{array}$ & -- & 405 & -- & -- & -- & -- & -- & & -- & -- \\
\hline Sweetgum & $\begin{array}{l}401 \\
1,755\end{array}$ & -- & -- & -- & -- & $\because$ & -- & -- & 451 & -- & -- \\
\hline Tupelo & 3,776 & -. & 1,291 & $1, \overline{102}$ & $\begin{array}{r}488 \\
1,121\end{array}$ & 720 & - & -- & 341 & 206 & -- \\
\hline Black cherry & 20,465 & - & 8,275 & $\begin{array}{l}1,102 \\
2,786\end{array}$ & $\begin{array}{l}1,121 \\
2,490\end{array}$ & $5 \overline{--}$ & -- & & 262 & -- & -- \\
\hline Black walnut & 21,262 & -- & 7,302 & 5,623 & $\begin{array}{l}2,490 \\
2,280\end{array}$ & $\begin{array}{l}2,593 \\
1,790\end{array}$ & $-\overline{-}$ & 2,155 & 1,375 & 791 & -- \\
\hline Butternut & 1,107 & -- & 1,107 & & 2,000 & $\begin{array}{r}1,790 \\
.-\end{array}$ & 845 & 1,134 & 1,553 & 417 & 318 \\
\hline Yellow-poplar & 12,416 & -- & 3,823 & 2,419 & 409 & 2,532 & $\overline{140}$ & 159 & $8 \overline{0}$ & -- & $\ddot{306}$ \\
\hline Persimmon & & -- & - & & & -- & 140 & $\begin{aligned} 159 \\
-.\end{aligned}$ & 1,850 & 688 & 396 \\
\hline Sassafras & 9,855 & -- & 2,095 & 1,093 & 1,960 & 1,823 & 909 & -- & 1,360 & 615 & $\because$ \\
\hline Other hardwoods & 18,493 & -- & 5,565 & 84 & 2,891 & 1,932 & 1,825 & -- & $\begin{array}{r}1,500 \\
496\end{array}$ & $\begin{array}{r}615 \\
--\end{array}$ & $\begin{array}{l}-- \\
--\end{array}$ \\
\hline Total & 503,055 & -- & 96,501 & 80,663 & 65,766 & 61,151 & 48,801 & 33,813 & 62,132 & 37,660 & 16,568 \\
\hline All species & 511,194 & 4,540 & 98,682 & 81,220 & 65,766 & 61,650 & 48,801 & 34,175 & 62,132 & 37,660 & 16,568 \\
\hline
\end{tabular}

1/International $/ 4$-inch rule. 
Table 57.--Net annual growth of growing stock and sawtimber on timberland by softwoods and hardwoods and Forest Survey unit, Indiana, 1966 and 1985

\begin{tabular}{|c|c|c|c|c|}
\hline \multirow[b]{2}{*}{ Species group } & \multicolumn{2}{|c|}{ Growing stock } & \multicolumn{2}{|c|}{ Sawt imber } \\
\hline & $1966^{1 /}$ & 1985 & $1966^{1 /}$ & 1985 \\
\hline & \multicolumn{2}{|c|}{ Thousand cubic feet } & Thous and & board feet 21 \\
\hline $\begin{array}{l}\text { Softwoods } \\
\text { Hardwoods }\end{array}$ & $\begin{array}{r}4,446 \\
99,099 \\
\end{array}$ & $\begin{array}{r}6,638 \\
146,997 \\
\end{array}$ & $\begin{array}{r}11,613 \\
258,332 \\
\end{array}$ & $\begin{array}{r}39,303 \\
686,641 \\
\end{array}$ \\
\hline All species & 103,545 & 153,635 & 269,945 & 725,944 \\
\hline $\begin{array}{l}\text { Lower Wabash } \\
\text { Softwoods } \\
\text { Hardwoods }\end{array}$ & $\begin{array}{r}637 \\
17,573 \\
\end{array}$ & $\begin{array}{r}1,108 \\
31,034\end{array}$ & $\begin{array}{r}183 \\
40,518 \\
\end{array}$ & $\begin{array}{r}5,599 \\
148,202 \\
\end{array}$ \\
\hline All species & 18,210 & 32,142 & 40,701 & 153,801 \\
\hline $\begin{array}{l}\text { Knobs Unit } \\
\text { Softwoods } \\
\text { Hardwoods }\end{array}$ & $\begin{array}{r}2,929 \\
39,758 \\
\end{array}$ & $\begin{array}{r}4,013 \\
64,933 \\
\end{array}$ & $\begin{array}{r}6,305 \\
94,041 \\
\end{array}$ & $\begin{array}{r}24,785 \\
321,337 \\
\end{array}$ \\
\hline All species & 42,687 & 68,946 & 100,346 & 346,122 \\
\hline $\begin{array}{l}\text { Upland Flats } \\
\text { Softwoods } \\
\text { Hardwoods }\end{array}$ & $\begin{array}{r}10 \\
7,036 \\
\end{array}$ & $\begin{array}{r}856 \\
15,911 \\
\end{array}$ & $\begin{array}{r}77 \\
15,763 \\
\end{array}$ & $\begin{array}{r}713 \\
61,647 \\
\end{array}$ \\
\hline All species & 7,046 & 16,767 & 15,840 & 62,360 \\
\hline $\begin{array}{l}\text { Northern Unit } \\
\text { So ftwoods } \\
\text { Hardwoods }\end{array}$ & $\begin{array}{r}870 \\
34,732 \\
\end{array}$ & $\begin{array}{r}661 \\
35,119 \\
\end{array}$ & $\begin{array}{r}5,048 \\
108,010 \\
\end{array}$ & $\begin{array}{r}8,206 \\
155,455 \\
\end{array}$ \\
\hline All species & 35,602 & 35,780 & 113,058 & 163,661 \\
\hline
\end{tabular}

1/Figures have been adjusted from those published after the 1967 survey to conform to 1985 volumes because of changes in survey procedures.

2/ International $1 / 4$-inch rule. 
Table 58.--Net annual growth of growing stock on timberland by species group and ownership class, Indiana, 1985

(In thous and cubic feet)

\begin{tabular}{|c|c|c|c|c|c|c|c|c|c|}
\hline \multirow[b]{2}{*}{ Species group } & \multirow[b]{2}{*}{$\begin{array}{c}\text { All } \\
\text { owners }\end{array}$} & \multicolumn{8}{|c|}{ Ownership class } \\
\hline & & $\begin{array}{c}\text { National } \\
\text { forest }\end{array}$ & $\begin{array}{c}\text { Misc. } \\
\text { federal }\end{array}$ & State & $\begin{array}{l}\text { County \& } \\
\text { municipal }\end{array}$ & $\begin{array}{l}\text { Forest } \\
\text { industry }\end{array}$ & Farmer & $\begin{array}{l}\text { Misc. } \\
\text { priv.- } \\
\text { corp. }\end{array}$ & $\begin{array}{l}\text { Misc. } \\
\text { priv.- } \\
\text { indiv. }\end{array}$ \\
\hline \multicolumn{10}{|l|}{ So ftwoods } \\
\hline Jack pine & 135 & -. & - & 24 & -. & -- & 19 & 8 & 84 \\
\hline Red pine & 198 & -- & -- & 12 & -. & -- & 87 & 74 & 25 \\
\hline White pine & 1,600 & 137 & -- & 251 & -- & -- & 412 & 435 & 365 \\
\hline Shortleaf pine & -85 & -383 & 5 & - & -- & -- & 88 & 133 & 72 \\
\hline other yellow pines & 1,990 & -5 & -- & 138 & -- & -- & 601 & 415 & 841 \\
\hline Tamarack & & -- & -- & 2 & -- & -- & -- & - & -- \\
\hline Baldcypress & 138 & -- & -- & - & -- & -- & -- & 58 & 80 \\
\hline Eastern redcedar & 2,133 & 13 & 11 & -7 & 8 & -- & 1,127 & 4 & 977 \\
\hline other softwoods & 527 & - & - & 145 & -- & -- & 382 & - & -- \\
\hline Total & 6,638 & -238 & 16 & 565 & 8 & -- & 2,716 & 1,127 & 2,444 \\
\hline \multicolumn{10}{|l|}{ Hardwoods } \\
\hline Select white oak & 15,350 & 915 & 886 & 1,365 & 102 & 121 & 5,232 & 931 & 5,798 \\
\hline Other white oak & 2,425 & 262 & 45 & 484 & 42 & 22 & 309 & 147 & 1,114 \\
\hline Select red oak & 8,276 & 200 & 272 & 334 & 39 & 55 & 3,242 & 772 & 3,362 \\
\hline other red oak & 14,388 & 533 & 696 & 966 & 50 & 111 & 5,216 & 1,091 & 5,725 \\
\hline select nickory & 5,583 & 43 & 217 & 115 & 17 & 26 & 2,117 & 392 & 2,656 \\
\hline other hickory & 6,402 & 227 & 287 & 216 & 17 & 36 & 2,680 & 311 & 2,628 \\
\hline Basswood & 1,693 & 4 & 90 & 133 & -42 & - & 761 & 133 & 614 \\
\hline Beech & 1,504 & 34 & -2 & 63 & -- & 18 & 762 & 123 & 506 \\
\hline Hard maple & 13,833 & 432 & 618 & 427 & 18 & 50 & 4,850 & 1,306 & 6,132 \\
\hline Soft maple & 12,287 & 94 & 590 & 683 & 108 & -- & 5,753 & 1,355 & 3,704 \\
\hline Elm & 4,654 & 14 & 4 & -5 & 94 & 83 & 2,244 & 560 & 1,660 \\
\hline Ash & 10,079 & 301 & 225 & 230 & 82 & -- & 4,473 & 1,102 & 3,666 \\
\hline Sycamore & 6,232 & 380 & 242 & 285 & 13 & -- & 2,556 & 654 & 2,102 \\
\hline Cottonwood & 3,789 & -- & 14 & 89 & 191 & -- & 2,493 & 603 & 399 \\
\hline Willow & 204 & -- & -15 & - & -22 & -- & 47 & 29 & 165 \\
\hline Hackberry & 2,319 & -- & 21 & 29 & 2 & -- & 1,262 & 142 & 863 \\
\hline Aspen & 728 & 114 & 128 & 6 & -- & -- & 109 & 166 & 205 \\
\hline Birch & 450 & 1 & 9 & 8 & -- & - & 136 & 40 & 255 \\
\hline Sweetgum & 3,203 & 12 & 39 & 110 & -- & -- & 1,163 & 383 & 1,496 \\
\hline Tupelo & 1,134 & 46 & 34 & 59 & -- & -34 & 461 & 72 & 496 \\
\hline Black cherry & 2,961 & 17 & 49 & 72 & 85 & -11 & 1,350 & 154 & 1,245 \\
\hline Black walnut & 3,959 & -36 & 113 & 66 & 29 & 4 & 1,935 & 397 & 1,451 \\
\hline Butternut & 3 & -- & -- & -- & 5 & - & -10 & 22 & -14 \\
\hline Yellow-poplar & 20,032 & 383 & 1,197 & 704 & 91 & 13 & 7,201 & 1,791 & 8,652 \\
\hline Persimmon & 423 & 1 & 6 & 3 & - & -- & 175 & 36 & 202 \\
\hline Sassafras & 3,295 & 458 & 17 & 120 & - & 44 & 705 & 130 & 1,821 \\
\hline other hardwoods & 1,791 & 23 & 91 & -21 & 18 & - & 663 & 251 & 766 \\
\hline Total & 146,997 & 4,458 & 5,873 & 6,541 & 939 & 538 & 57,885 & 13,093 & 57,670 \\
\hline All species & 153,635 & 4,220 & 5,889 & 7,106 & 947 & 538 & 60,601 & 14,220 & 60,114 \\
\hline
\end{tabular}


Table 59.--Net annual growth of sawtimber on timberland by species group and ownership class, lndiana, 1985

( In thousand board feet) 1 /

\begin{tabular}{|c|c|c|c|c|c|c|c|c|c|}
\hline \multirow[b]{2}{*}{ Species group } & \multirow[b]{2}{*}{$\begin{array}{c}\text { All } \\
\text { owners }\end{array}$} & \multicolumn{8}{|c|}{ Ownership class } \\
\hline & & $\begin{array}{l}\text { National } \\
\text { forest }\end{array}$ & $\begin{array}{l}\text { Misc. } \\
\text { federal }\end{array}$ & State & $\begin{array}{l}\text { County \& } \\
\text { municipal }\end{array}$ & $\begin{array}{l}\text { Forest } \\
\text { industry }\end{array}$ & Farmer & $\begin{array}{l}\text { Misc. } \\
\text { priv.- } \\
\text { corp. }\end{array}$ & $\begin{array}{l}\text { Misc. } \\
\text { priv.- } \\
\text { indiv. }\end{array}$ \\
\hline \multicolumn{10}{|l|}{ So ft woods } \\
\hline Jack pine & 479 & -- & -- & 253 & -- & -- & 5 & -- & 221 \\
\hline Red pine & 4,432 & -- & -- & 45 & -- & -- & 3,424 & 891 & 72 \\
\hline White pine & 14,553 & 6,264 & -- & 1,010 & -- & -- & 3,131 & 2,470 & 1,678 \\
\hline Shortleaf pine & 2,520 & 944 & 25 & -- & -- & -- & 223 & 654 & 674 \\
\hline Other yellow pines & 6,884 & -- & -- & 698 & -- & -- & 1,792 & 744 & 3,650 \\
\hline Tamarack & -131 & -- & -- & -131 & -- & -- & -- & -- & -- \\
\hline Baldcypress & 744 & -- & -- & -- & -- & -- & -- & 311 & 433 \\
\hline Eastern redcedar & 6,237 & 80 & 16 & -45 & -- & $-\infty$ & 1,865 & 26 & 4,295 \\
\hline Other softwoods & 3,585 & -- & -- & -- & -- & -- & 3,585 & -- & -- \\
\hline Total & 39,303 & 7,288 & 41 & 1,830 & -- & -- & 14,025 & 5,096 & 11,023 \\
\hline \multicolumn{10}{|l|}{ Hardwoods } \\
\hline Select white oak & 79,020 & 6,335 & 4,524 & 5,916 & 726 & 949 & 25,313 & 9,527 & 25,730 \\
\hline Other white oak & 13,109 & 1,774 & 237 & 2,272 & 177 & 57 & 1,878 & 601 & 6,113 \\
\hline Select red oak & 50,361 & 1,511 & 1,958 & 1,720 & 189 & 412 & 24,294 & 3,890 & 16,387 \\
\hline other red oak & 77,947 & 3,468 & 3,114 & 4,259 & 208 & 1,044 & 28,585 & 5,348 & 31,921 \\
\hline Select hickory & 28,196 & 366 & 1,340 & 603 & 82 & 90 & 9,380 & 1,441 & 14,894 \\
\hline Other hickory & 35,440 & 2,034 & 2,069 & 775 & 16 & 208 & 12,870 & 1,666 & 15,802 \\
\hline Basswood & 9,019 & - & 1,756 & 719 & 36 & -- & 2,737 & 857 & 2,914 \\
\hline Beech & 5,676 & 23 & -6 & 311 & - & 53 & 2,968 & 601 & 1,726 \\
\hline Hard maple & 59,429 & 1,354 & 1,915 & 385 & 91 & 175 & 19,788 & 4,091 & 31,630 \\
\hline Soft maple & 36,743 & 143 & 1,591 & 1,228 & 963 & -- & 18,839 & 2,438 & 11,541 \\
\hline Elm & 6,401 & -16 & -151 & 273 & -13 & -118 & 3,665 & 948 & 1,813 \\
\hline Ash & 49,560 & 1,296 & 502 & 1,454 & 592 & -- & 20,818 & 9,015 & 15,883 \\
\hline Sycamore & 31,449 & 584 & 1,450 & 558 & 43 & -- & 12,476 & 5,964 & 10,374 \\
\hline Cottonwood & 19,464 & -- & 8 & 491 & 1,181 & -- & 11,335 & 3,450 & 2,999 \\
\hline Willow & 378 & -- & -64 & -- & -- & -- & -574 & -40 & 1,056 \\
\hline Hackberry & 10,351 & -- & 110 & 320 & 11 & -- & 4,546 & 1,000 & 4,364 \\
\hline Aspen & 5,517 & 262 & 390 & 16 & -- & -- & 690 & 3,439 & 720 \\
\hline Birch & 1,262 & 9 & 22 & 48 & -- & - & 403 & -- & 780 \\
\hline Sweetgum & 11,577 & 105 & 203 & 361 & -- & -- & 5,600 & 210 & 5,098 \\
\hline Tupelo & 5,201 & 96 & 16 & 235 & -- & -188 & 1,426 & 541 & 3,075 \\
\hline Bl ack cherry & 16,699 & 94 & 148 & 203 & 69 & -58 & 10,391 & 896 & 4,956 \\
\hline Black walnut & 19,928 & 44 & 774 & 474 & 132 & 6 & 10,086 & 2,083 & 6,329 \\
\hline Butternut & 46 & -- & -- & -- & 28 & -- & -84 & 145 & -43 \\
\hline Yellow-poplar & 90,476 & 1,509 & 4,560 & 2,921 & 82 & 38 & 30,400 & 7,811 & 43,155 \\
\hline Persimmon & 2,198 & -- & -- & -- & -- & -- & 710 & 180 & 1,308 \\
\hline Sassafras & 7,878 & 36 & 312 & -24 & -- & 104 & 3,011 & 394 & 4,045 \\
\hline other hardwoods & 13,316 & 114 & 395 & -25 & -- & -- & 7,384 & 398 & 5,050 \\
\hline Total & 686,641 & 21,141 & 27,173 & 25,493 & 4,613 & 2,772 & 268,935 & 66,894 & 269,620 \\
\hline All species & 725,944 & 28,429 & 27,214 & 27,323 & 4,613 & 2,772 & 282,960 & 71,990 & 280,643 \\
\hline
\end{tabular}

1/ International $1 / 4$-inch rule. 


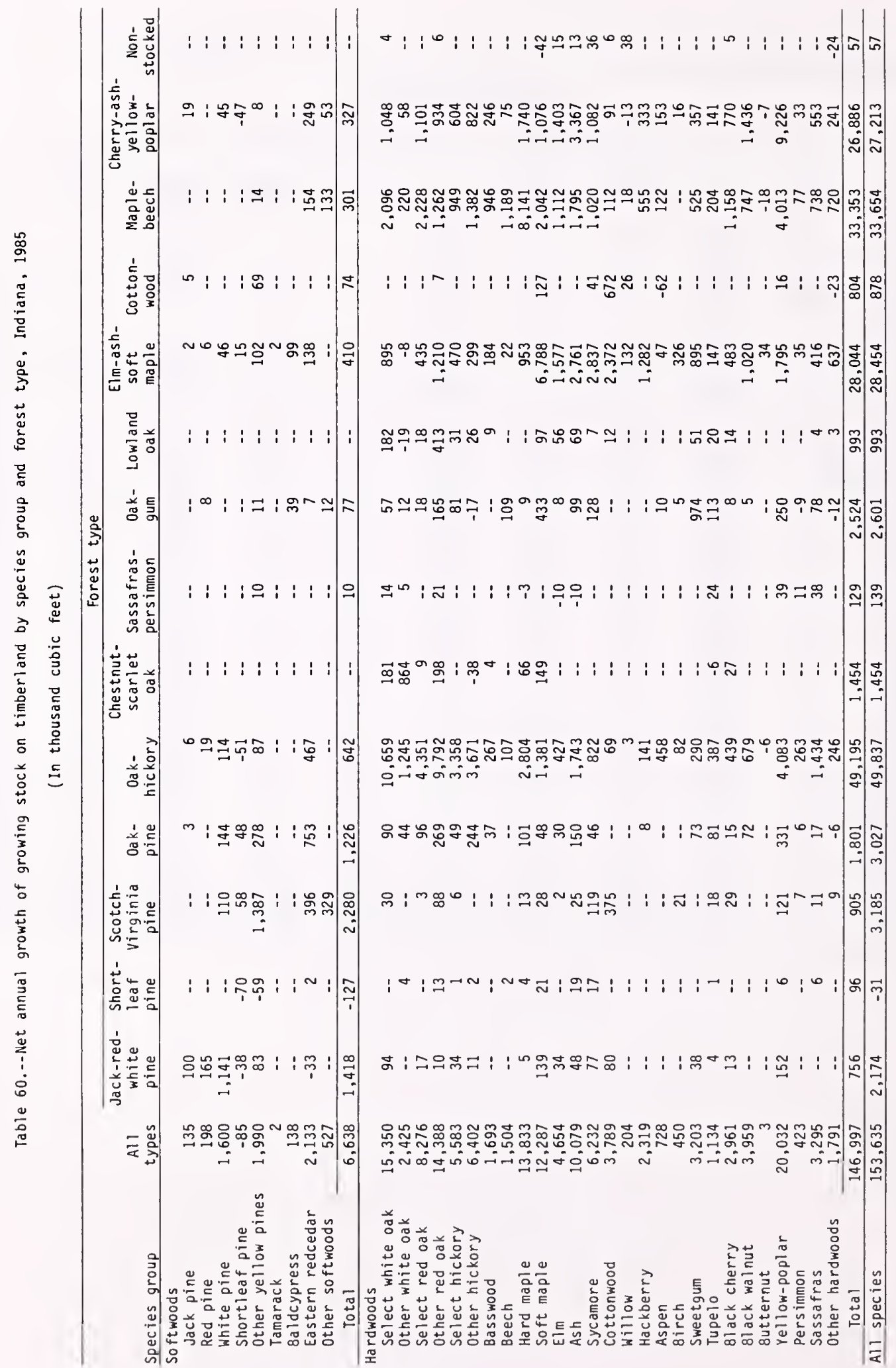




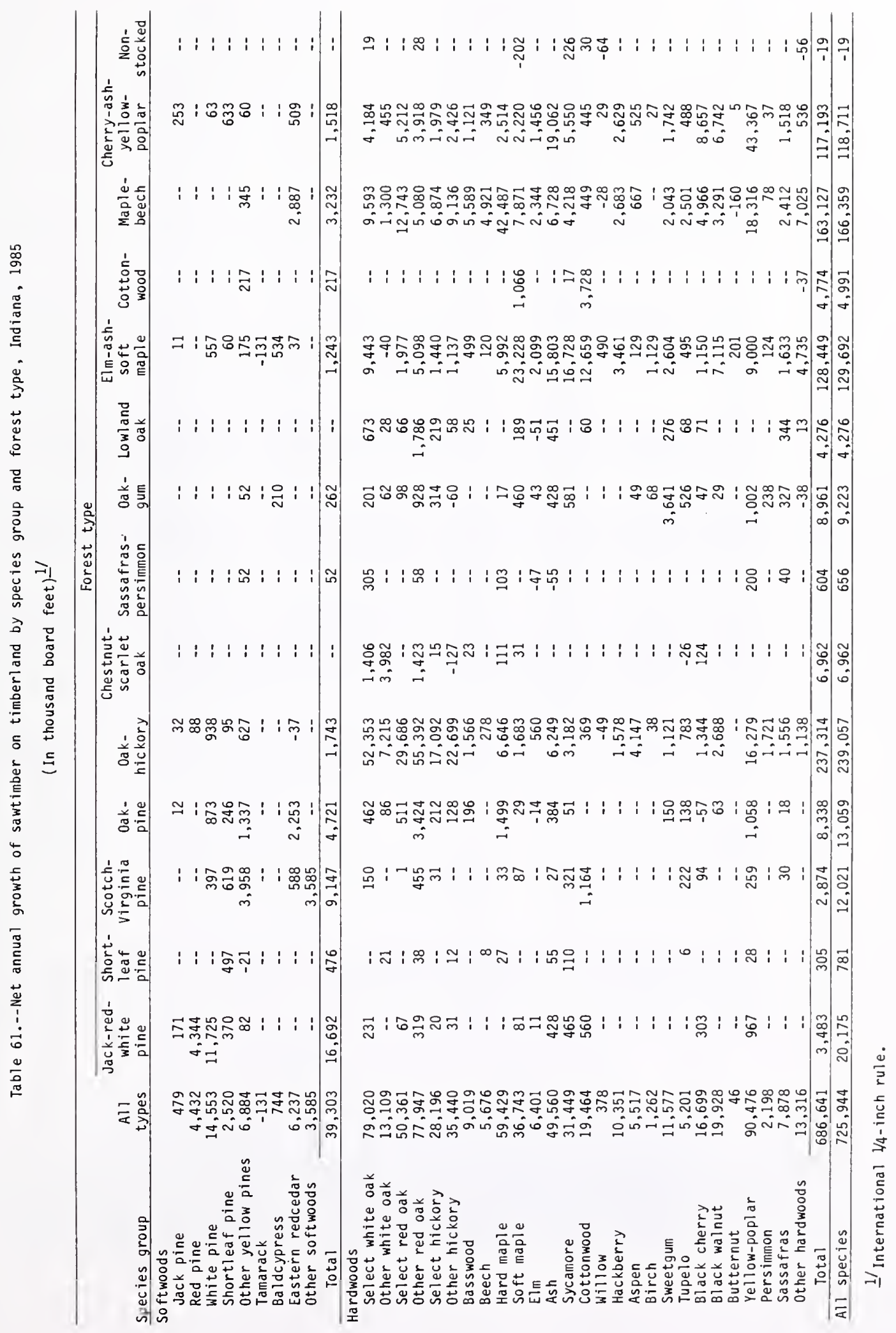




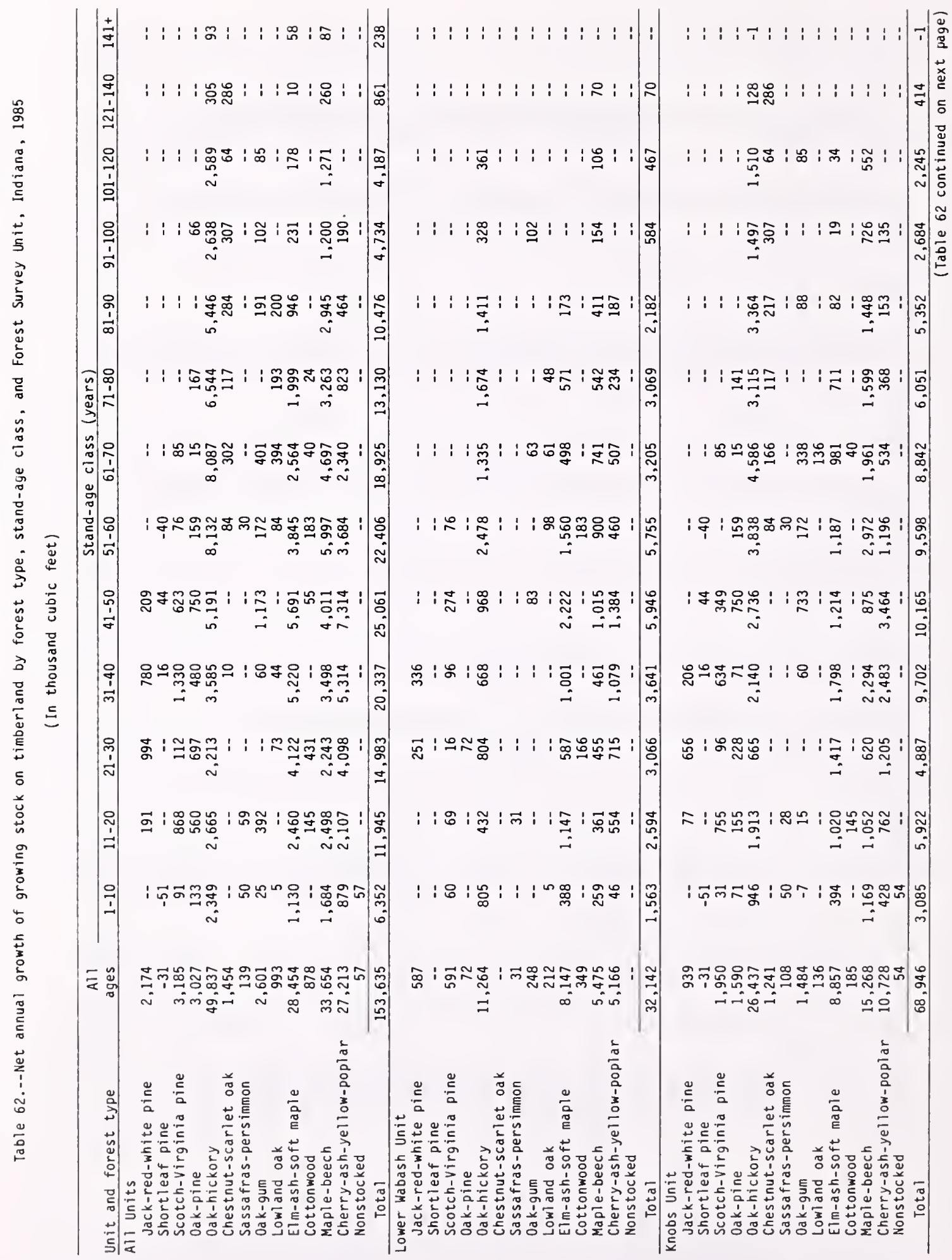




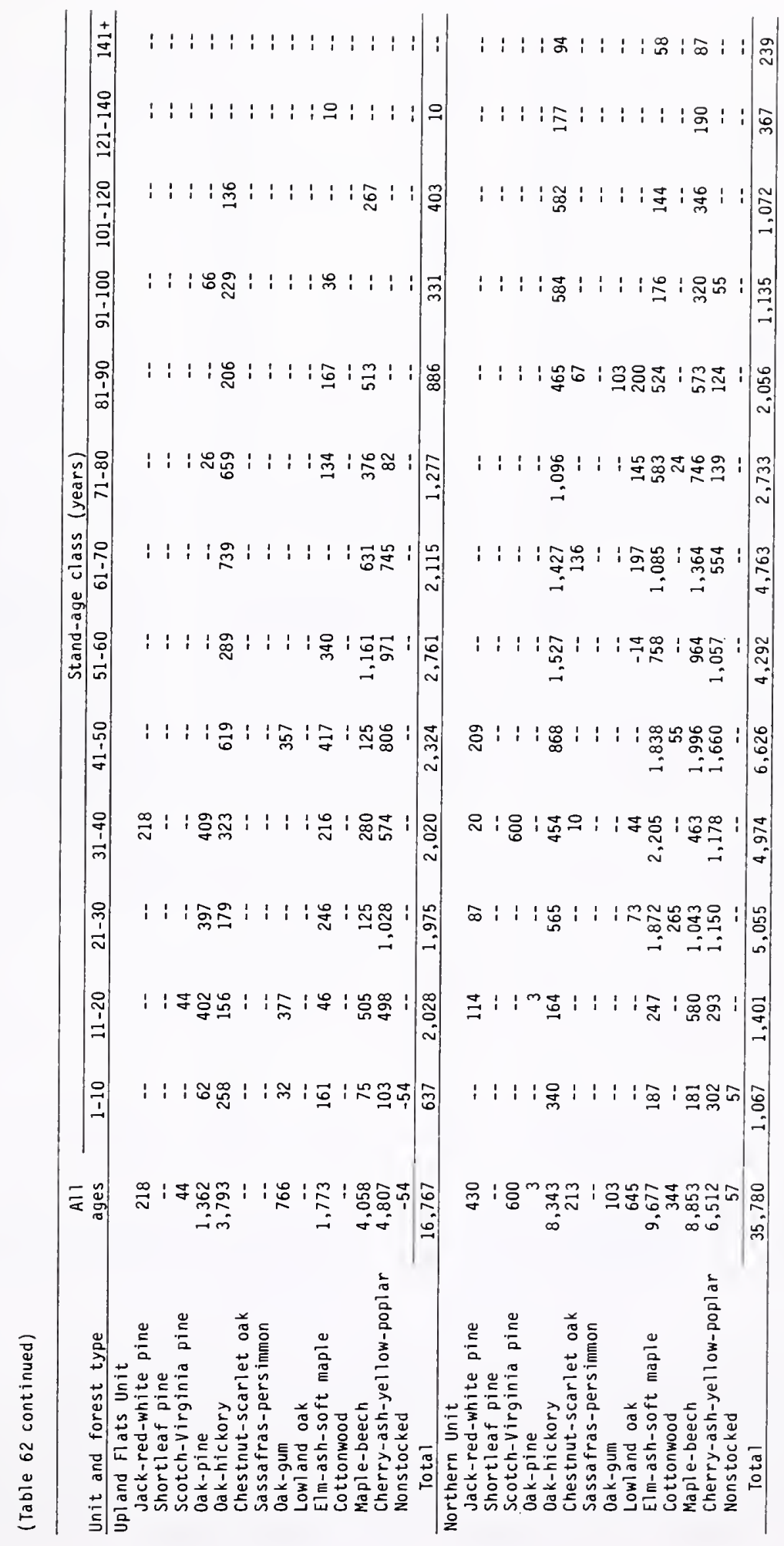




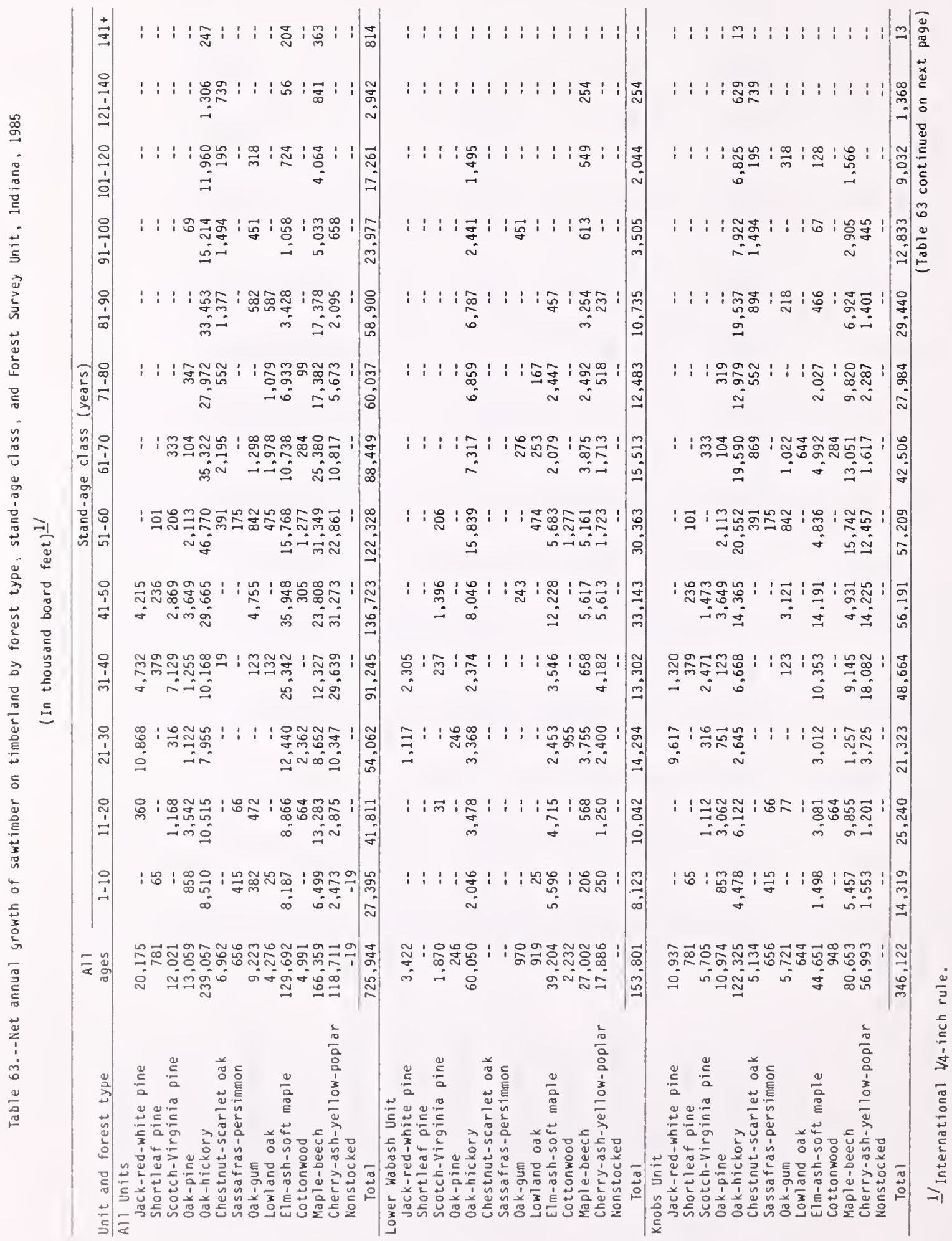




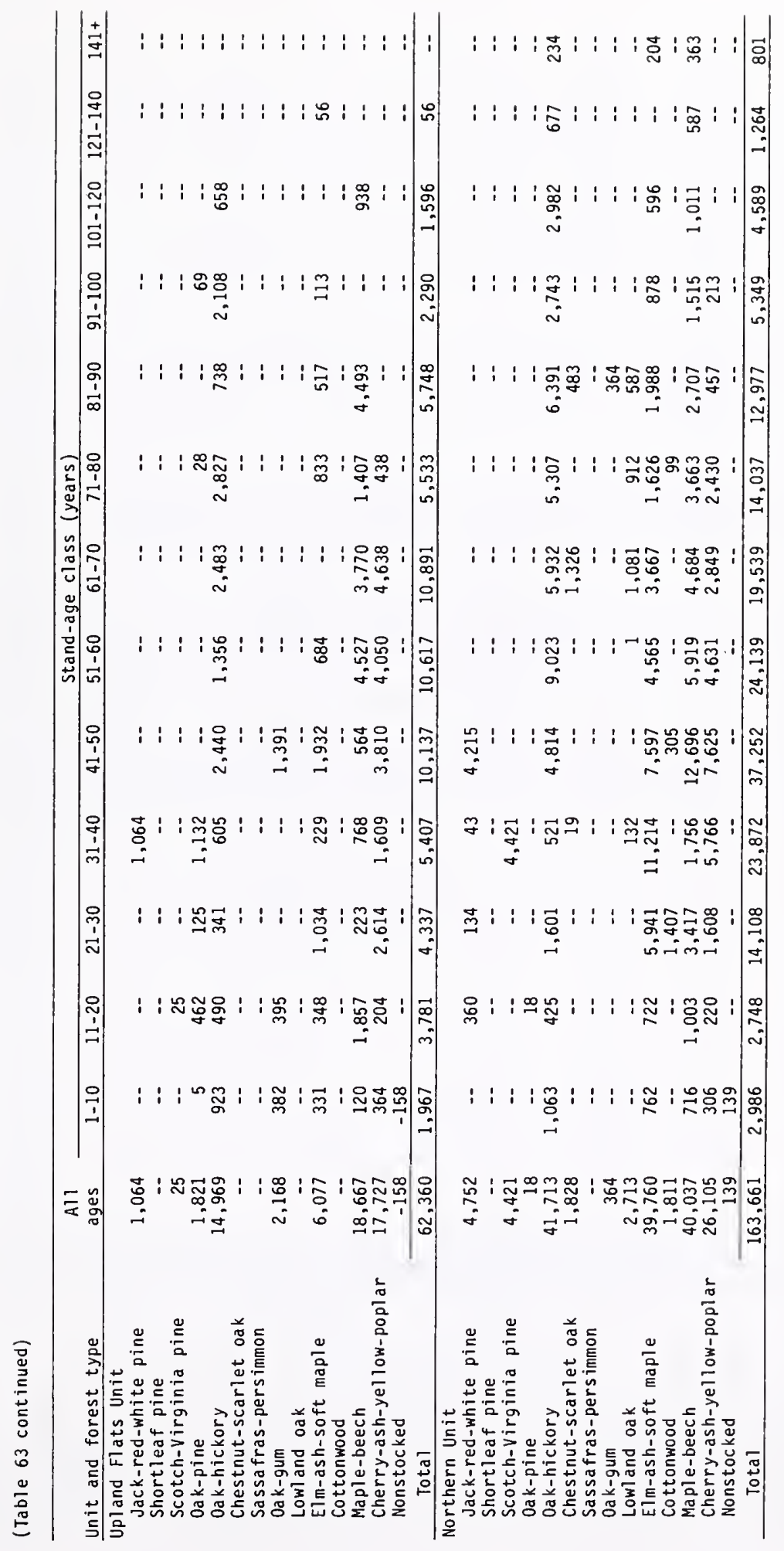




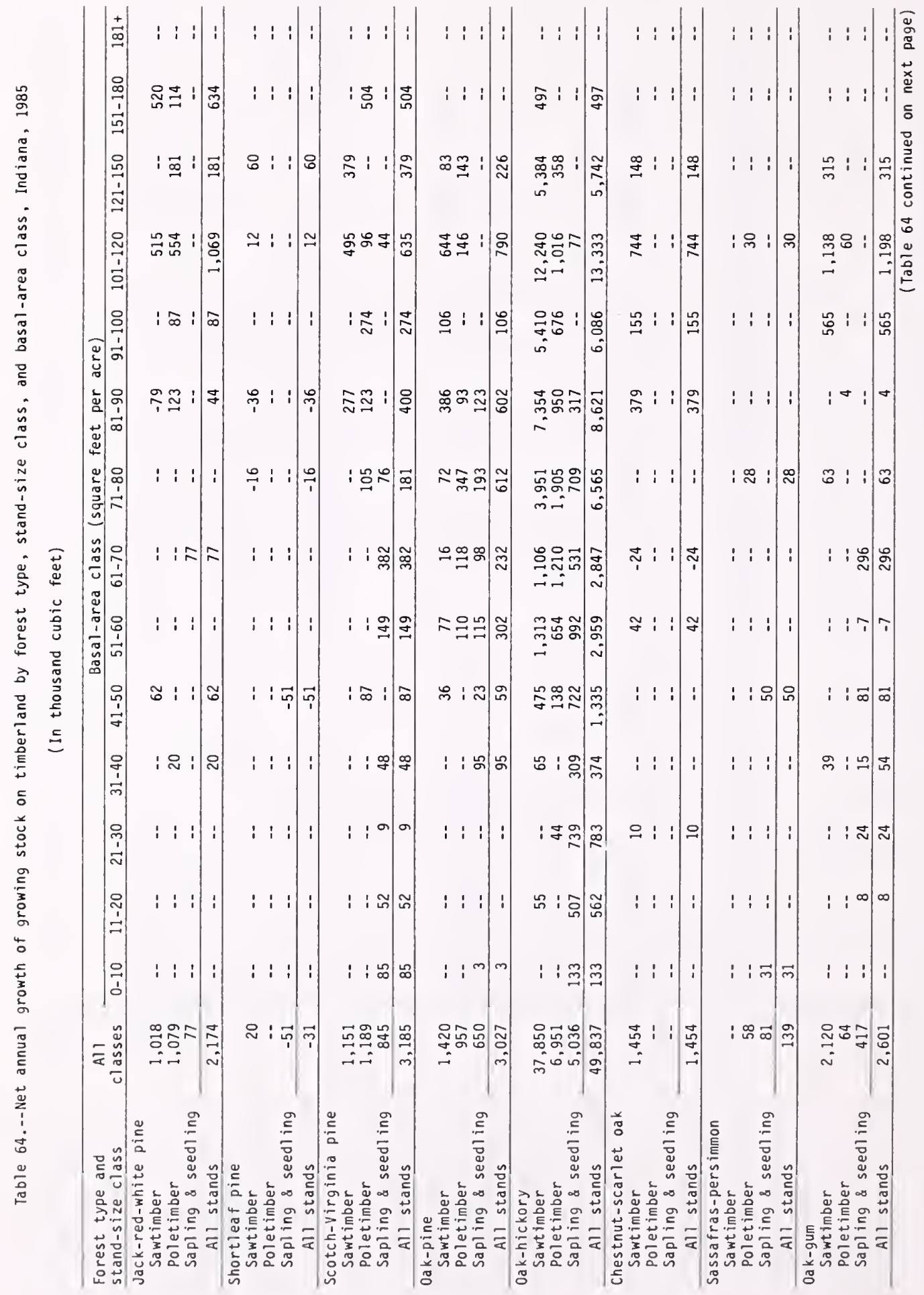




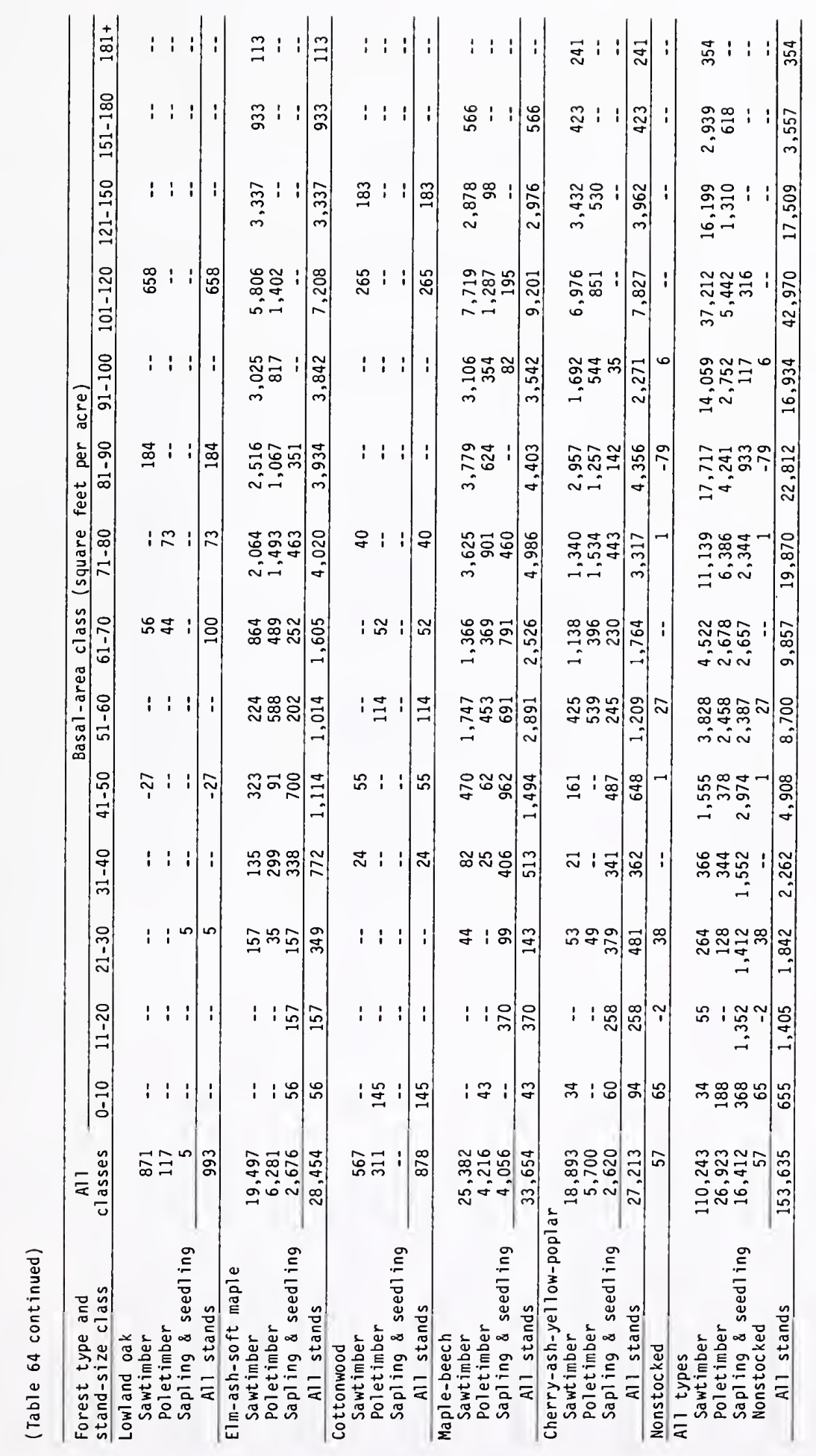




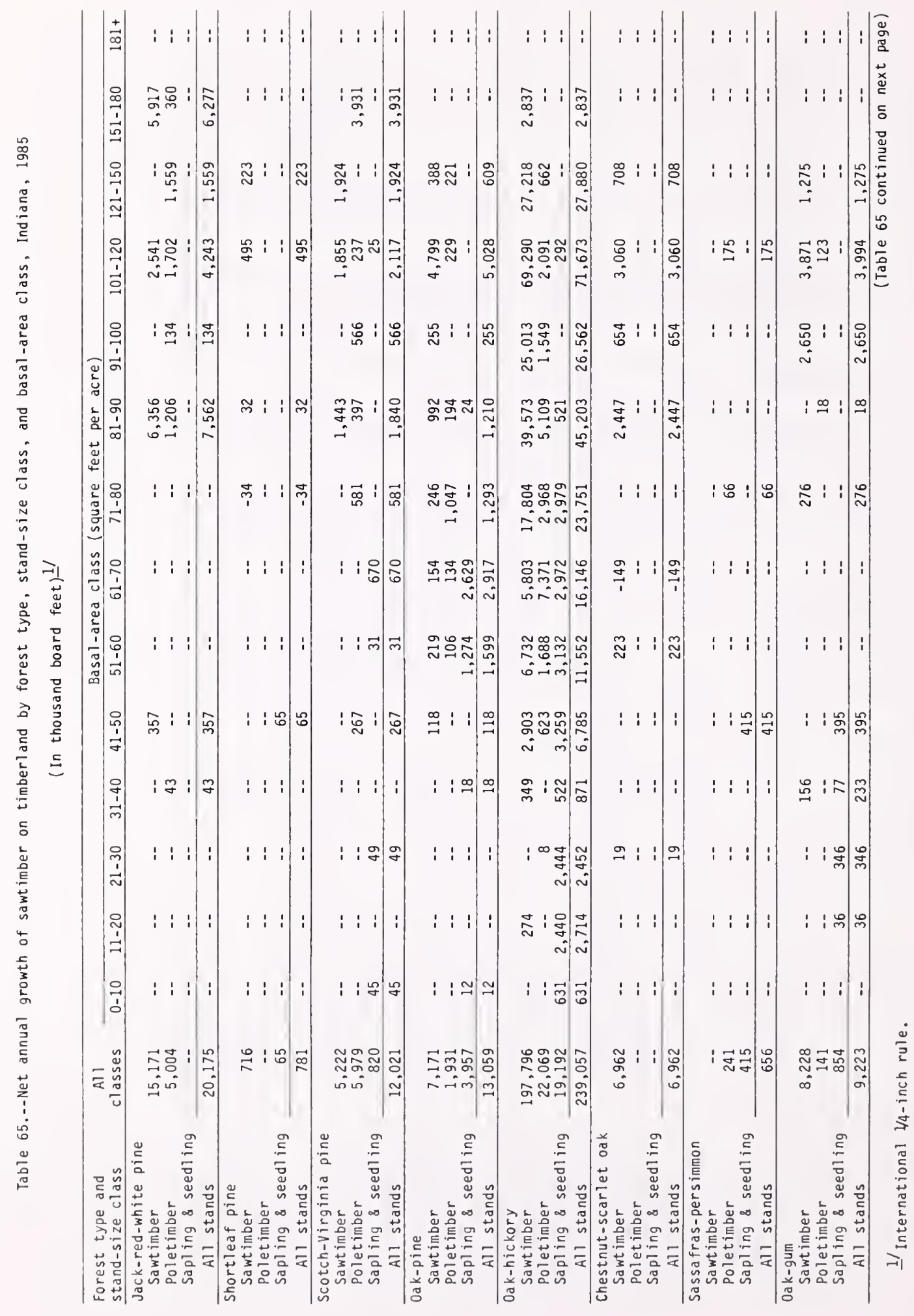




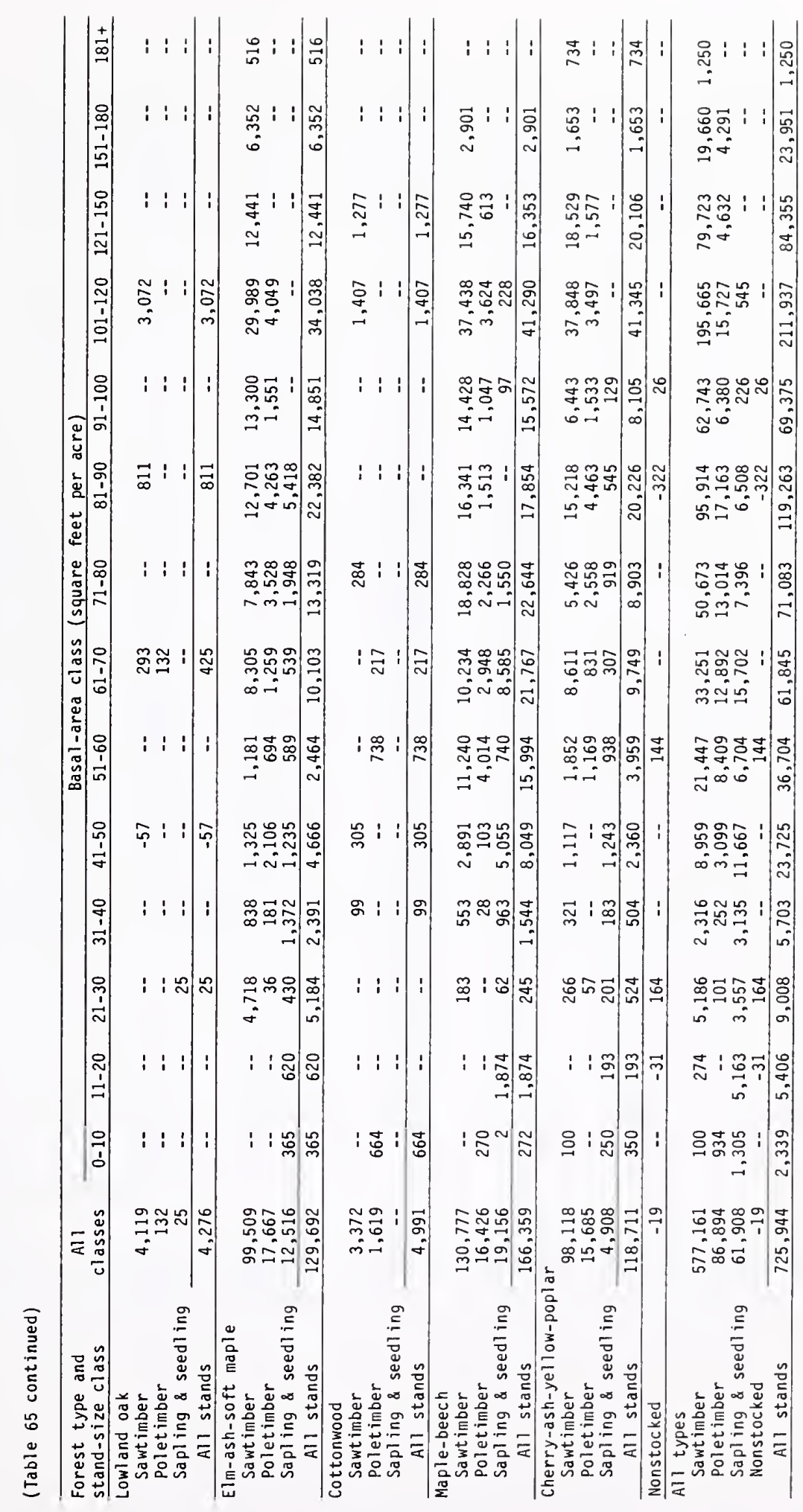


Table 66.--Net annual growth of growing stock and sawtimber on timberland by county and species group, Indiana, 1985

\begin{tabular}{|c|c|c|c|c|c|c|c|c|c|c|}
\hline \multirow[b]{2}{*}{$\begin{array}{l}\text { Unit } \\
\text { and county }\end{array}$} & \multicolumn{5}{|c|}{ Growing stock } & \multicolumn{5}{|c|}{ Sawt imber } \\
\hline & $\begin{array}{c}\text { All } \\
\text { species }\end{array}$ & Pine & $\begin{array}{c}\text { Other } \\
\text { softwoods }\end{array}$ & $\begin{array}{c}\text { Soft } \\
\text { hardwoods }\end{array}$ & $\begin{array}{c}\text { Hard } \\
\text { hardwoods } \\
\end{array}$ & $\begin{array}{c}\text { All } \\
\text { species }\end{array}$ & Pine & $\begin{array}{c}\text { Other } \\
\text { softwoods }\end{array}$ & $\begin{array}{c}\text { Soft } \\
\text { hardwoods }\end{array}$ & $\begin{array}{c}\text { Hard } \\
\text { hardwoods }\end{array}$ \\
\hline $\begin{array}{l}\text { Lower Wabash } \\
\text { Clay } \\
\text { Daviess } \\
\text { Gibson } \\
\text { Greene } \\
\text { Knox } \\
\text { Martin } \\
\text { Parke } \\
\text { Pike } \\
\text { Posey } \\
\text { Putnam } \\
\text { Sullivan } \\
\text { Vanderburgh } \\
\text { Vermillion } \\
\text { Vigo }\end{array}$ & $\begin{array}{r}\ldots \\
1,635 \\
1,522 \\
1,536 \\
3,875 \\
1,043 \\
4,673 \\
3,544 \\
3,278 \\
1,728 \\
2,811 \\
2,473 \\
917 \\
1,247 \\
1,860 \\
\end{array}$ & $\begin{array}{r}105 \\
40 \\
53 \\
193 \\
12 \\
19 \\
145 \\
110 \\
36 \\
30 \\
93 \\
20 \\
22 \\
54\end{array}$ & $\begin{array}{r}\text { lousand cub } \\
20 \\
9 \\
10 \\
33 \\
8 \\
6 \\
20 \\
15 \\
9 \\
9 \\
17 \\
4 \\
6 \\
10 \\
\end{array}$ & $\begin{array}{r}696 \\
714 \\
746 \\
1,768 \\
446 \\
1,911 \\
1,753 \\
1,598 \\
772 \\
1,098 \\
1,157 \\
409 \\
529 \\
903 \\
\end{array}$ & $\begin{array}{r}814 \\
759 \\
727 \\
1,881 \\
577 \\
2,737 \\
1,626 \\
1,555 \\
911 \\
1,674 \\
1,206 \\
484 \\
690 \\
893 \\
\end{array}$ & $\begin{array}{r}\ldots \\
\ldots, \\
8,293 \\
7,045 \\
6,931 \\
17,306 \\
5,265 \\
23,816 \\
16,252 \\
14,694 \\
8,591 \\
16,013 \\
11,208 \\
4,367 \\
5,783 \\
8,237 \\
\end{array}$ & $\begin{array}{r}464 \\
227 \\
307 \\
986 \\
46 \\
61 \\
926 \\
694 \\
211 \\
61 \\
528 \\
106 \\
102 \\
309 \\
\end{array}$ & \begin{tabular}{|r|} 
sand bo \\
83 \\
20 \\
27 \\
140 \\
5 \\
8 \\
81 \\
60 \\
19 \\
10 \\
62 \\
11 \\
17 \\
28 \\
\end{tabular} & $\begin{array}{r}\text { feet } \\
2,808 \\
2,716 \\
2,756 \\
6,394 \\
1,834 \\
8,168 \\
6,626 \\
5,969 \\
3,169 \\
5,024 \\
4,384 \\
1,550 \\
2,023 \\
3,301 \\
\end{array}$ & $\begin{array}{r} \\
4,938 \\
4,082 \\
3,841 \\
9,786 \\
3,380 \\
15,579 \\
8,619 \\
7,971 \\
5,192 \\
10,918 \\
6,234 \\
2,700 \\
3,641 \\
4,599 \\
\end{array}$ \\
\hline Total & 32,142 & 932 & 176 & 14,500 & 16,534 & 153,801 & 5,028 & 571 & 56,722 & 91,480 \\
\hline $\begin{array}{l}\text { Knobs Unit } \\
\text { Brown } \\
\text { Clark } \\
\text { Crawford } \\
\text { Dubois } \\
\text { Floyd } \\
\text { Harrison } \\
\text { Jackson } \\
\text { Lawrence } \\
\text { Monroe } \\
\text { Morgan } \\
\text { Orange } \\
\text { Owen } \\
\text { Perry } \\
\text { Scott } \\
\text { Spencer } \\
\text { Warrick } \\
\text { Washington }\end{array}$ & $\begin{array}{l}5,261 \\
3,452 \\
4,600 \\
3,727 \\
1,394 \\
5,593 \\
4,692 \\
4,999 \\
4,811 \\
3,499 \\
4,946 \\
4,219 \\
5,441 \\
1,763 \\
2,477 \\
3,279 \\
4,793 \\
\end{array}$ & $\begin{array}{r}212 \\
245 \\
77 \\
186 \\
56 \\
275 \\
128 \\
113 \\
143 \\
130 \\
81 \\
180 \\
66 \\
101 \\
136 \\
196 \\
287 \\
\end{array}$ & $\begin{array}{r}89 \\
68 \\
78 \\
69 \\
17 \\
150 \\
138 \\
56 \\
85 \\
73 \\
103 \\
95 \\
76 \\
42 \\
67 \\
118 \\
77 \\
\end{array}$ & $\begin{array}{r}1,893 \\
1,292 \\
1,767 \\
1,369 \\
505 \\
2,232 \\
1,932 \\
1,776 \\
1,830 \\
1,396 \\
1,904 \\
1,537 \\
1,970 \\
689 \\
963 \\
1,397 \\
1,729 \\
\end{array}$ & $\begin{array}{r}3,067 \\
1,847 \\
2,678 \\
2,103 \\
816 \\
2,936 \\
2,494 \\
3,054 \\
2,753 \\
1,900 \\
2,858 \\
2,407 \\
3,329 \\
931 \\
1,311 \\
1,568 \\
2,700 \\
\end{array}$ & $\begin{array}{r}27,360 \\
17,252 \\
22,989 \\
19,040 \\
7,961 \\
25,693 \\
21,718 \\
26,324 \\
23,521 \\
16,010 \\
25,061 \\
22,905 \\
29,490 \\
8,198 \\
11,357 \\
14,014 \\
27,229 \\
\end{array}$ & $\begin{array}{r}1,640 \\
933 \\
1,442 \\
740 \\
254 \\
1,294 \\
1,381 \\
1,269 \\
661 \\
491 \\
2,060 \\
822 \\
2,681 \\
376 \\
545 \\
624 \\
1,381 \\
\end{array}$ & $\begin{array}{l}538 \\
349 \\
412 \\
401 \\
126 \\
442 \\
322 \\
502 \\
475 \\
275 \\
372 \\
476 \\
409 \\
157 \\
232 \\
257 \\
446 \\
\end{array}$ & $\begin{array}{l}9,031 \\
6,795 \\
7,487 \\
6,514 \\
2,364 \\
9,949 \\
7,452 \\
7,973 \\
7,720 \\
6,196 \\
7,995 \\
6,963 \\
8,394 \\
3,165 \\
4,254 \\
5,875 \\
8,178 \\
\end{array}$ & $\begin{array}{r}16,151 \\
9,175 \\
13,648 \\
11,385 \\
5,217 \\
14,008 \\
12,563 \\
16,580 \\
14,665 \\
9,048 \\
14,634 \\
14,644 \\
18,006 \\
4,500 \\
6,326 \\
7,258 \\
17,224 \\
\end{array}$ \\
\hline Total & 68,946 & 2,612 & 1,401 & 26,181 & 38,752 & 346,122 & 18,594 & 6,191 & 116,305 & 205,032 \\
\hline $\begin{array}{l}\text { Upland Flats U } \\
\text { Dearborn } \\
\text { Fayette } \\
\text { Frankl in } \\
\text { Jefferson } \\
\text { Jennings } \\
\text { Ohio } \\
\text { Ripley } \\
\text { Switzerland } \\
\text { Union }\end{array}$ & $\begin{array}{r}2,699 \\
897 \\
2,175 \\
2,402 \\
2,615 \\
808 \\
2,202 \\
2,414 \\
555 \\
\end{array}$ & $\begin{array}{r}5 \\
1 \\
7 \\
97 \\
4 \\
1 \\
58 \\
5 \\
1 \\
\end{array}$ & $\begin{array}{r}121 \\
19 \\
97 \\
114 \\
102 \\
38 \\
99 \\
64 \\
23 \\
\end{array}$ & $\begin{array}{r}1,100 \\
387 \\
856 \\
899 \\
1,092 \\
329 \\
808 \\
925 \\
229 \\
\end{array}$ & $\begin{array}{r}1,473 \\
490 \\
1,215 \\
1,292 \\
1,417 \\
440 \\
1,237 \\
1,420 \\
302 \\
\end{array}$ & $\begin{array}{l}9,668 \\
2,997 \\
7,406 \\
9,598 \\
9,405 \\
2,843 \\
8,791 \\
9,774 \\
1,878 \\
\end{array}$ & $\begin{array}{r}11 \\
8 \\
19 \\
271 \\
13 \\
3 \\
164 \\
8 \\
4 \\
\end{array}$ & $\begin{array}{r}31 \\
-12 \\
18 \\
58 \\
23 \\
8 \\
59 \\
22 \\
5 \\
\end{array}$ & $\begin{array}{r}4,327 \\
1,323 \\
3,077 \\
3,991 \\
4,273 \\
1,250 \\
3,552 \\
4,181 \\
832 \\
\end{array}$ & $\begin{array}{l}5,299 \\
1,678 \\
4,292 \\
5,278 \\
5,096 \\
1,582 \\
5,016 \\
5,563 \\
1,037\end{array}$ \\
\hline Total & 16,767 & 179 & 677 & 6,625 & 9,286 & 62,360 & 501 & 212 & 26,806 & 34,841 \\
\hline
\end{tabular}

1/International $1 / 4$-inch rule. 
(Table 66 continued)

\begin{tabular}{|c|c|c|c|c|c|c|c|c|c|c|}
\hline \multirow[b]{2}{*}{$\begin{array}{l}\text { Unit } \\
\text { and county }\end{array}$} & \multicolumn{5}{|c|}{ Growing stock } & \multicolumn{5}{|c|}{ Sawt imber } \\
\hline & $\begin{array}{c}\text { All } \\
\text { species }\end{array}$ & Pine & $\begin{array}{c}\text { Dther } \\
\text { softwoods }\end{array}$ & $\begin{array}{l}\text { So ft } \\
\text { hardwoods }\end{array}$ & $\begin{array}{c}\text { Hard } \\
\text { hardwoods }\end{array}$ & $\begin{array}{c}\text { All } \\
\text { species }\end{array}$ & Pine & $\begin{array}{c}\text { Other } \\
\text { so ftwoods }\end{array}$ & $\begin{array}{c}\text { Soft } \\
\text { hardwoods }\end{array}$ & $\begin{array}{c}\text { Hard } \\
\text { hardwoods }\end{array}$ \\
\hline Northern Unit & -- & $-\cdot-I$ & lousand cub & feet - - & ---- & $--\cdot$ & $-\cdots I$ & usand boar & feet $1 /$ - & $--\cdot-$ \\
\hline Adams & 462 & 7 & -- & 200 & 255 & 2,175 & 86 & -2 & 785 & 1,306 \\
\hline Allen & 832 & 19 & 1 & 378 & 434 & 3,442 & 225 & -- & 1,209 & 2,008 \\
\hline Barthol omew & 1,434 & 13 & 1 & 597 & 823 & 6,962 & 159 & -8 & 2,468 & 4,343 \\
\hline Benton & 56 & 1 & -- & 24 & 31 & 253 & 12 & -- & 94 & 147 \\
\hline Blackford & 310 & 1 & -- & 126 & 183 & 1,563 & 19 & -2 & 557 & 989 \\
\hline Boone & 467 & 2 & -- & 193 & 272 & 2,191 & 24 & -3 & 776 & 1,394 \\
\hline Carroll & 585 & 7 & -- & 250 & 328 & 2,726 & 92 & -3 & 983 & 1,654 \\
\hline Cass & 788 & 20 & -- & 359 & 409 & 3,588 & 261 & -2 & 1,320 & 2,009 \\
\hline clinton & 292 & 1 & -- & 116 & 175 & 1,401 & 13 & -2 & 486 & 904 \\
\hline Decatur & 796 & 4 & 1 & 322 & 469 & 3,891 & 62 & -5 & 1,377 & 2,457 \\
\hline De Kalb & 901 & 25 & -- & 417 & 459 & 4,041 & 330 & -2 & 1,526 & 2,187 \\
\hline Delaware & 496 & 11 & -- & 225 & 260 & 2,171 & 144 & -1 & 801 & 1,227 \\
\hline El khart & 909 & 18 & 1 & 410 & 480 & 4,005 & 219 & -2 & 1,460 & 2,328 \\
\hline Fountain & 1,236 & 31 & 1 & 560 & 644 & 5,711 & 412 & -4 & 2,125 & 3,178 \\
\hline Fulton & 658 & 15 & -- & 294 & 349 & 3,022 & 189 & -2 & 1,105 & 1,730 \\
\hline Grant & 625 & 8 & -- & 266 & 351 & 2,995 & 120 & -3 & 1,094 & 1,784 \\
\hline Hamil ton & 651 & 13 & 1 & 298 & 339 & 2,858 & 186 & -1 & 1,070 & 1,603 \\
\hline Hancock & 392 & 11 & -- & 181 & 200 & 1,747 & 150 & -1 & 663 & 935 \\
\hline Hendricks & 531 & 6 & -- & 225 & 300 & 2,482 & 62 & -3 & 872 & 1,551 \\
\hline Henry & 648 & 11 & -- & 285 & 352 & 2,967 & 141 & -3 & 1,084 & 1,745 \\
\hline Howard & 237 & 3 & -- & 104 & 130 & 964 & 39 & - & 336 & 589 \\
\hline Huntington & 709 & 19 & -- & 319 & 371 & 3,073 & 244 & -1 & 1,105 & 1,725 \\
\hline Jasper & 834 & 15 & 1 & 370 & 448 & 3,741 & 173 & -2 & 1,343 & 2,227 \\
\hline Jay & 793 & 3 & 1 & 319 & 470 & 3,989 & 43 & -6 & 1,406 & 2,546 \\
\hline Johnson & 633 & 3 & 1 & 258 & 371 & 3,106 & 37 & -4 & 1,100 & 1,973 \\
\hline Kosciusko & 1,092 & 6 & 1 & 444 & 641 & 5,469 & 83 & -7 & 1,933 & 3,460 \\
\hline La Grange & 1,056 & 22 & - & 484 & 550 & 4,591 & 274 & -3 & 1,724 & 2,596 \\
\hline Lake & 589 & 11 & -- & 261 & 317 & 2,715 & 147 & -2 & 1,003 & 1,567 \\
\hline La Porte & 1,282 & 19 & 1 & 567 & 695 & 5,767 & 228 & -4 & 2,088 & 3,455 \\
\hline Mad ison & 432 & 5 & $\ldots$ & 181 & 246 & 2,073 & 70 & -2 & 739 & 1,266 \\
\hline Marion & 14 & -- & -- & 6 & 8 & 37 & -- & $=$ & 11 & 26 \\
\hline Marshall & 959 & 20 & -- & 425 & 514 & 4,441 & 237 & -3 & 1,595 & 2,612 \\
\hline Miami & 866 & 8 & 1 & 360 & 497 & 4,275 & 110 & -6 & 1,543 & 2,628 \\
\hline Montgomery & 803 & 22 & 1 & 372 & 408 & 3,503 & 314 & -1 & 1,328 & 1,862 \\
\hline Newton & 644 & 12 & - & 283 & 349 & 3,059 & 176 & -3 & 1,143 & 1,743 \\
\hline Noble & 1,016 & 24 & 1 & 463 & 528 & 4,480 & 322 & -2 & 1,656 & 2,504 \\
\hline Porter & 959 & 21 & 1 & 440 & 497 & 4,174 & 278 & -2 & 1,578 & 2,320 \\
\hline Pulaski & 879 & 20 & 1 & 396 & 462 & 3,978 & 260 & -2 & 1,463 & 2,257 \\
\hline Randol ph & 647 & 3 & -- & 258 & 386 & 3,155 & 42 & -4 & 1,105 & 2,012 \\
\hline Rush & 341 & 8 & -- & 153 & 180 & 1,407 & 92 & -- & 497 & 818 \\
\hline St. Joseph & 703 & 16 & 1 & 321 & 365 & 3,052 & 195 & -1 & 1,129 & 1,729 \\
\hline Shelby & 425 & 4 & -- & 177 & 244 & 2,085 & 59 & -3 & 758 & 1,271 \\
\hline Starke & 868 & 16 & 1 & 383 & 468 & 3,998 & 206 & -3 & 1,458 & 2,337 \\
\hline Steuben & 981 & 24 & 1 & 450 & 506 & 4,270 & 316 & -2 & 1,597 & 2,359 \\
\hline Ti ppecanoe & 738 & 22 & -- & 344 & 372 & 3,205 & 293 & -1 & 1,209 & 1,704 \\
\hline Tipton & 128 & 3 & -- & 57 & 68 & 519 & 34 & - & 187 & 298 \\
\hline Wabash & 747 & 17 & -- & 341 & 389 & 3,262 & 216 & -2 & 1,224 & 1,824 \\
\hline Warren & 761 & 12 & -- & 329 & 420 & 3,554 & 153 & -3 & 1,289 & $\begin{array}{l}1,0<4 \\
2,115\end{array}$ \\
\hline Wayne & 995 & 21 & -- & 446 & 528 & 4,545 & 270 & -3 & 1,658 & 2,620 \\
\hline Wells & 540 & 13 & -- & 246 & 281 & 2,393 & 180 & -1 & 902 & 1,312 \\
\hline White & 392 & 10 & -- & 183 & 199 & 1,652 & 130 & -- & 619 & 903 \\
\hline Whitley & 648 & 16 & -- & 295 & 337 & 2,938 & 203 & -2 & 1,077 & 1,660 \\
\hline Total & 35,780 & 642 & 19 & 15,761 & 19,358 & 163,661 & 8,330 & -124 & 59,658 & 95,797 \\
\hline Al] counties & 153,635 & 4,365 & 2,273 & 63,067 & 83,930 & 725,944 & 32,453 & 6,850 & 259,491 & 427,150 \\
\hline
\end{tabular}

$1 /$ International $1 / 4$-inch rule. 
Table 67.--Current annual timber removals from growing stock and sawtimber on timberland by species group, Indiana, 1966 and 1985

\begin{tabular}{|c|c|c|c|c|}
\hline \multirow[b]{2}{*}{ Species group } & \multicolumn{2}{|c|}{ Growing stock } & \multicolumn{2}{|c|}{ Sawtimber } \\
\hline & 1966 & 1985 & 1966 & 1985 \\
\hline \multirow[b]{2}{*}{$\begin{array}{l}\text { Softwoods } \\
\text { Pine } \\
\text { 8aldcypress } \\
\text { Other softwoods }\end{array}$} & \multicolumn{2}{|c|}{ Thousand cubic feet } & \multicolumn{2}{|c|}{ Thousand board feet $1 /$} \\
\hline & $\begin{array}{r}142 \\
164 \\
94 \\
\end{array}$ & $\begin{array}{r}184 \\
119 \\
\end{array}$ & $\begin{array}{r}297 \\
607 \\
96 \\
\end{array}$ & $\begin{array}{r}826 \\
\ldots \\
231 \\
\end{array}$ \\
\hline Total & 400 & 303 & 1,000 & 1,057 \\
\hline $\begin{array}{l}\text { Hardwoods } \\
\text { Select white oak } \\
\text { other white oak } \\
\text { Select red oak } \\
\text { 0ther red oak } \\
\text { Hickory } \\
\text { 8asswood } \\
\text { 8eech } \\
\text { Hard maple } \\
\text { Soft maple } \\
\text { Elm } \\
\text { Ash } \\
\text { Sycamore } \\
\text { Cottonwood } \\
\text { Aspen } \\
\text { Sweetgum } \\
\text { Tupelo } \\
81 \text { ack cherry } \\
81 \text { ack walnut } \\
\text { Yellow-poplar } \\
\text { 0ther hardwoods }\end{array}$ & $\begin{array}{r}7,056 \\
1,193 \\
5,998 \\
10,442 \\
3,485 \\
753 \\
3,588 \\
5,238 \\
4,613 \\
675 \\
3,500 \\
2,988 \\
4,258 \\
253 \\
1,515 \\
571 \\
424 \\
2,810 \\
3,902 \\
1,238 \\
\end{array}$ & $\begin{array}{r}12,310 \\
2,700 \\
9,976 \\
17,035 \\
7,022 \\
935 \\
3,426 \\
5,437 \\
3,150 \\
888 \\
7,781 \\
3,242 \\
1,920 \\
285 \\
1,049 \\
538 \\
1,519 \\
1,924 \\
9,663 \\
1,722\end{array}$ & $\begin{array}{r}37,744 \\
6,315 \\
33,206 \\
53,589 \\
15,479 \\
4,837 \\
18,252 \\
31,517 \\
21,838 \\
4,014 \\
18,886 \\
16,870 \\
20,481 \\
1,131 \\
5,902 \\
2,965 \\
2,782 \\
17,782 \\
24,625 \\
5,822 \\
\end{array}$ & $\begin{array}{r}61,583 \\
12,740 \\
51,242 \\
86,148 \\
33,678 \\
4,452 \\
17,612 \\
25,614 \\
15,098 \\
3,929 \\
36,840 \\
16,304 \\
11,072 \\
1,078 \\
5,410 \\
2,748 \\
7,761 \\
11,003 \\
50,667 \\
6,174 \\
\end{array}$ \\
\hline Total & 64,500 & 92,522 & 344,037 & 461,153 \\
\hline All species & 64,900 & 92,825 & 345,037 & 462,210 \\
\hline
\end{tabular}

1/ International $1 / 4$-inch rule. 
Table 68.--Average annual timber removals from growing stock and sawtimber on timberland by county and species group, Indiana, 1966-1985

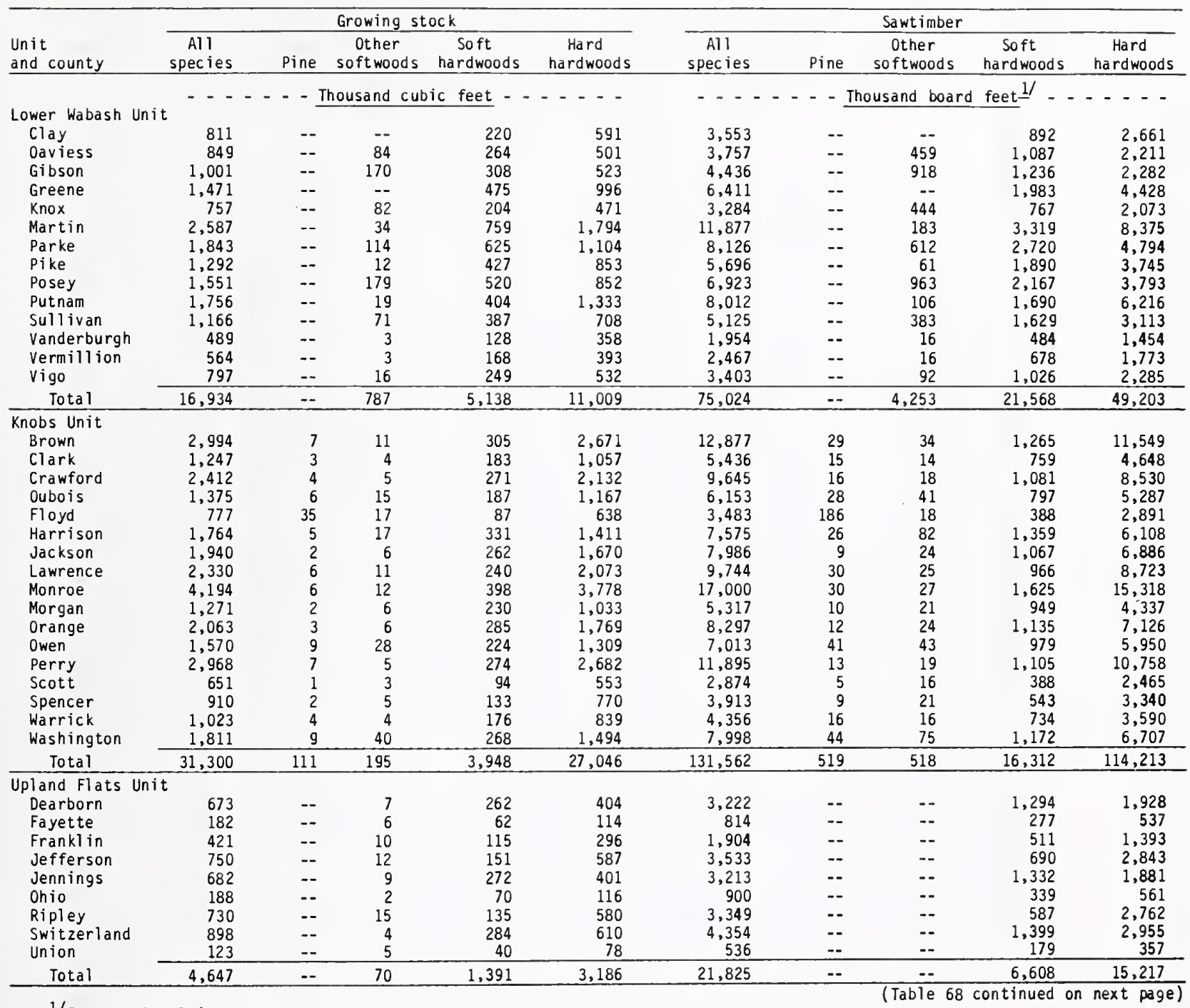

1/International $1 / 4$-inch rule. 
(Table 68 continued)

\begin{tabular}{|c|c|c|c|c|c|c|c|c|c|c|}
\hline \multirow[b]{2}{*}{$\begin{array}{l}\text { Unit } \\
\text { and county }\end{array}$} & \multicolumn{5}{|c|}{ Growing stock } & \multicolumn{5}{|c|}{ Sawt imber } \\
\hline & $\begin{array}{c}\text { All } \\
\text { species }\end{array}$ & Pine & $\begin{array}{c}\text { ather } \\
\text { softwoods }\end{array}$ & $\begin{array}{c}\text { Soft } \\
\text { hardwoods }\end{array}$ & $\begin{array}{c}\text { Hard } \\
\text { hardwoods }\end{array}$ & $\begin{array}{c}\text { All } \\
\text { species }\end{array}$ & Pine & $\begin{array}{c}\text { Other } \\
\text { softwoods }\end{array}$ & $\begin{array}{c}\text { Soft } \\
\text { hardwoods }\end{array}$ & $\begin{array}{c}\text { Hard } \\
\text { hardwoods }\end{array}$ \\
\hline Northern Unit & -- & --1 & rousand cub & ic feet - & ---- & $-\cdots$ & -1 & us and Doar & feet $1 /$ - & $-\cdots$ \\
\hline Adams & 257 & -- & -- & 76 & 181 & 1,132 & -- & -- & 327 & 805 \\
\hline Allen & 599 & 1 & -- & 218 & 380 & 2,596 & -- & -- & 905 & 1,691 \\
\hline Barthol omew & 846 & - & -- & 265 & 581 & 3,803 & - & -- & 1,167 & 2,636 \\
\hline Benton & 56 & - & -- & 16 & 40 & 208 & $\ldots$ & $\rightarrow$ & 60 & 148 \\
\hline Bl ack ford & 190 & -- & -- & 59 & 131 & 857 & -- & -- & 262 & 595 \\
\hline Boone & 335 & -- & -- & 107 & 228 & 1,490 & -- & -- & 469 & 1,021 \\
\hline Carroll & 355 & -- & -- & 107 & 248 & 1,572 & -- & - & 462 & 1,110 \\
\hline Cass & 320 & 1 & -- & 91 & 228 & 1,363 & -- & -- & 366 & 997 \\
\hline Clinton & 241 & -- & -- & 64 & 177 & 1,047 & -- & -- & 279 & 768 \\
\hline Decatur & 549 & 1 & -- & 158 & 390 & 2,455 & -- & -- & 703 & 1,752 \\
\hline De Kalb & 318 & 1 & -- & 91 & 226 & 1,345 & -- & -- & 359 & 986 \\
\hline Delaware & 278 & - & -- & 97 & 181 & 1,174 & - & -- & 396 & 778 \\
\hline Elkhart & 486 & 1 & -- & 130 & 355 & 2,128 & -- & -- & 533 & 1,595 \\
\hline Fount a in & 475 & 1 & -- & 138 & 336 & 2,064 & - & -- & 566 & 1,498 \\
\hline Fulton & 303 & - & -- & 94 & 209 & 1,323 & -- & -- & 395 & 928 \\
\hline Grant & 394 & -- & -- & 117 & 277 & 1,724 & -- & - & 497 & 1,227 \\
\hline Hamilton & 324 & -- & -- & 91 & 233 & 1,383 & -- & -- & 367 & 1,016 \\
\hline Hancock & 160 & -- & -- & 48 & 112 & 660 & -- & -- & 183 & 477 \\
\hline Hendricks & 320 & -- & -- & 99 & 221 & 1,414 & -- & -- & 430 & 984 \\
\hline Henry & 351 & -- & -- & 107 & 244 & 1,513 & -- & -- & 447 & 1,066 \\
\hline Howard & 235 & -- & -- & 90 & 145 & 1,022 & - & -- & 384 & 638 \\
\hline Huntington & 315 & 1 & -- & 107 & 207 & 1,339 & -- & -- & 434 & 905 \\
\hline Jasper & 460 & - & -- & 133 & 327 & 2,014 & -- & -- & 559 & 1,455 \\
\hline Jay & 504 & - & -- & 152 & 352 & 2,283 & -- & -- & 678 & 1,605 \\
\hline Johnson & 400 & -- & -- & 126 & 274 & 1,801 & - & -- & 557 & 1,244 \\
\hline Kosciusko & 689 & 1 & -- & 212 & 476 & 3,107 & -- & -- & 941 & 2,166 \\
\hline La Grange & 422 & 1 & -- & 122 & 299 & 1,789 & -- & -- & 492 & 1,297 \\
\hline Lake & 425 & - & -- & 127 & 298 & 1,768 & -- & - & 498 & 1,270 \\
\hline La Porte & 735 & 1 & -- & 202 & 532 & 3,232 & -- & -- & 850 & 2,382 \\
\hline Madison & 311 & -- & -- & 91 & 220 & 1,344 & -- & -- & 386 & 958 \\
\hline Marion & 463 & -- & -- & 146 & 317 & 1,928 & - & -- & 554 & 1,374 \\
\hline Marshall & 432 & -- & -- & 128 & 304 & 1,903 & - & -- & 541 & 1,362 \\
\hline Miami & 502 & -- & -- & 157 & 345 & 2,252 & - & -- & 691 & 1,561 \\
\hline Montgomery & 372 & 1 & -- & 129 & 242 & 1,566 & - & -- & 518 & 1,048 \\
\hline Newton & 316 & - & -- & 99 & 217 & 1,364 & -- & -- & 415 & 949 \\
\hline Noble & 476 & 1 & -- & 160 & 315 & 2,049 & -- & -- & 664 & 1,385 \\
\hline Porter & 472 & 1 & -- & 133 & 338 & 1,958 & -- & -- & 520 & 1,438 \\
\hline Pulaski. & 405 & 1 & -- & 121 & 283 & 1,772 & - & - & 506 & 1,266 \\
\hline Randolph & 487 & - & -- & 129 & 358 & 2,161 & -- & -- & 570 & 1,591 \\
\hline Rush & 227 & -- & -- & 82 & 145 & 977 & - & -- & 346 & 631 \\
\hline St. Joseph & 419 & 1 & -- & 114 & 304 & 1,777 & -- & -- & 449 & 1,328 \\
\hline Shelby & 250 & - & -- & 77 & 173 & 1,097 & - & -- & 332 & 765 \\
\hline Starke & 427 & -- & -- & 125 & 302 & 1,898 & - & - & 529 & 1,369 \\
\hline Steuben & 445 & 1 & - & 152 & 292 & 1,919 & - & -- & 624 & 1,295 \\
\hline Tippecanoe & 333 & 1 & -- & 111 & 221 & 1,387 & - & -- & 439 & 948 \\
\hline Tipton & 95 & - & -- & 34 & 61 & 399 & - & -- & 138 & 261 \\
\hline Wabash & 340 & 1 & -- & 102 & 237 & 1,418 & - & -- & 406 & 1,012 \\
\hline Warren & 411 & - & -- & 131 & 280 & 1,832 & -- & - & 566 & 1,266 \\
\hline Wayne & 471 & 1 & -- & 137 & 333 & 2,042 & - & -- & 564 & 1,478 \\
\hline Wells & 263 & - & -- & 89 & 174 & 1,104 & -- & -- & 360 & 744 \\
\hline White & 196 & $=$ & -- & 58 & 138 & 792 & $\ldots$ & -- & $22 b$ & 567 \\
\hline Whitley & 254 & -- & -- & 71 & 183 & 1,085 & -- & -- & 286 & 799 \\
\hline Total & 19,709 & 19 & -- & 6,020 & 13,670 & 85,630 & - & -- & 25,195 & 60,435 \\
\hline All counties & 72,590 & 130 & 1,052 & 16,497 & 54,911 & 314,041 & 519 & 4,771 & 69,683 & 239,068 \\
\hline
\end{tabular}

1/International 1/4-inch rule. 
Table 69.--Average annual removals of growing stock on timberland by species group and Forest Survey Unit, Indiana, 1966-1985

(In thousand cubic feet per year)

\begin{tabular}{|c|c|c|c|c|c|}
\hline \multirow[b]{2}{*}{ Species group } & \multirow[b]{2}{*}{$\begin{array}{c}\text { All } \\
\text { Units }\end{array}$} & \multicolumn{4}{|c|}{ Forest Survey Unit } \\
\hline & & $\begin{array}{l}\text { Lower } \\
\text { Wabash }\end{array}$ & Knobs & $\begin{array}{l}\text { Upland } \\
\text { Flats }\end{array}$ & Northern \\
\hline \multicolumn{6}{|l|}{ So ftwoods } \\
\hline Shortleaf pine & 6 & -- & 6 & -- & -- \\
\hline other yellow pines & 124 & -- & 105 & -- & 19 \\
\hline 8aldcypress & 787 & 787 & -- & -- & -- \\
\hline Eastern redcedar & 265 & -- & 195 & 70 & -- \\
\hline Total & 1,182 & 787 & 306 & 70 & 19 \\
\hline \multicolumn{6}{|l|}{ Hardwoods } \\
\hline Select white oak & 13,618 & 2,191 & 7,439 & 932 & 3,056 \\
\hline Other white oak & 3,007 & 142 & 2,712 & -- & 153 \\
\hline Select red oak & 7,576 & 1,679 & 3,312 & 535 & 2,050 \\
\hline other red oak & 10,406 & 2,038 & 5,356 & 621 & 2,391 \\
\hline Select hickory & 3,544 & 1,127 & 1,692 & 129 & 596 \\
\hline other hickory & 3,395 & 1,275 & 1,580 & 112 & 428 \\
\hline Basswood & 679 & 19 & 136 & 13 & 511 \\
\hline Beech & 1,486 & 50 & 714 & 289 & 433 \\
\hline Hard maple & 4,589 & 836 & 2,019 & 220 & 1,514 \\
\hline So ft maple & 2,685 & 1,202 & 410 & 342 & 731 \\
\hline Elm & 1,348 & 413 & 419 & 25 & 491 \\
\hline Ash & 4,724 & 875 & 1,502 & 289 & 2,058 \\
\hline Sycamore & 2,224 & 778 & 546 & 37 & 863 \\
\hline Cottonwood & 1,485 & 77 & 39 & 10 & 1,359 \\
\hline Willow & 302 & 116 & 52 & - & 134 \\
\hline Hackberry & 499 & 33 & 74 & 24 & 368 \\
\hline Aspen & 386 & 21 & 32 & 65 & 268 \\
\hline $8 \mathrm{irch}$ & 465 & 109 & 273 & -- & 83 \\
\hline Sweetgum & 701 & 325 & 191 & 162 & 23 \\
\hline Tupelo & 375 & 133 & 150 & 92 & -- \\
\hline 81 ack cherry & 1,115 & 230 & 301 & 104 & 480 \\
\hline 81 ack walnut & 1,600 & 384 & 321 & 53 & 842 \\
\hline 8utternut & 82 & 58 & 24 & -- & -- \\
\hline Yellow-poplar & 3,813 & 1,486 & 1,194 & 497 & 636 \\
\hline Persimmon & 58 & 27 & 31 & -- & -- \\
\hline Sassafras & 615 & 198 & 294 & 20 & 103 \\
\hline Other hardwoods & 631 & 325 & 181 & 6 & 119 \\
\hline Total & 71,408 & 16,147 & 30,994 & 4,577 & 19,690 \\
\hline All species & 72,590 & 16,934 & 31,300 & 4,647 & 19,709 \\
\hline
\end{tabular}


Table 70.--Average annual removals of sawtimber on timberland by species group and Forest Survey Unit, Indiana, 1966-1985

(In thousand board feet per year) 1 '

\begin{tabular}{|c|c|c|c|c|c|}
\hline \multirow[b]{2}{*}{ Species group } & \multirow[b]{2}{*}{$\begin{array}{c}\text { All } \\
\text { Units }\end{array}$} & \multicolumn{4}{|c|}{ Forest Survey Unit } \\
\hline & & $\begin{array}{l}\text { Lower } \\
\text { Wabash }\end{array}$ & Knobs & $\begin{array}{l}\text { Upland } \\
\text { Flats }\end{array}$ & Northern \\
\hline \multicolumn{6}{|l|}{ So ftwoods } \\
\hline Shortleaf pine & -- & -- & -- & - & -- \\
\hline other yellow pines & 519 & -- & 519 & -- & -- \\
\hline Baldcypress & 4,253 & 4,253 & -- & -- & -- \\
\hline Eastern redcedar & 518 & -- & 518 & -- & -- \\
\hline Total & 5,290 & 4,253 & 1,037 & -- & -- \\
\hline \multicolumn{6}{|l|}{ Hardwoods } \\
\hline Select white oak & 61,606 & 9,836 & 32,619 & 4,668 & 14,483 \\
\hline Other white oak & 12,500 & 655 & 11,150 & -- & 695 \\
\hline Select red oak & 34,846 & 7,628 & 15,647 & 2,692 & 8,879 \\
\hline other red oak & 48,688 & 9,839 & 25,022 & 2,985 & 10,842 \\
\hline Select hickory & 13,819 & 5,155 & 6,121 & 551 & 1,992 \\
\hline other hickory & 13,031 & 5,419 & 5,620 & 499 & 1,493 \\
\hline Basswood & 3,066 & 99 & 640 & 71 & 2,256 \\
\hline Beech & 6,873 & 256 & 3,081 & 1,385 & 2,151 \\
\hline Hard maple & 18,650 & 3,251 & 7,250 & 945 & 7,204 \\
\hline Soft maple & 11,185 & 5,055 & 1,670 & 1,567 & 2,893 \\
\hline Elm & 4,346 & 1,357 & 1,404 & 63 & 1,522 \\
\hline Ash & 19,564 & 3,805 & 5,352 & 1,303 & 9,104 \\
\hline Sycamore & 10,474 & 3,705 & 2,535 & 144 & 4,090 \\
\hline Cottonwood & 7,240 & 372 & 77 & 51 & 6,740 \\
\hline Willow & 638 & 270 & 205 & -- & 163 \\
\hline Hackberry & 2,138 & 101 & 330 & 123 & 1,584 \\
\hline Aspen & 964 & 111 & -- & 337 & 516 \\
\hline Birch & 1,936 & 554 & 1,219 & -- & 163 \\
\hline Sweetgum & 3,230 & 1,603 & 821 & 721 & 85 \\
\hline Tupelo & 1,688 & 575 & 632 & 481 & -- \\
\hline Black cherry & 4,149 & 622 & 1,124 & 391 & 2,012 \\
\hline Black walnut & 6,550 & 1,927 & 1,221 & 189 & 3,213 \\
\hline Butternut & 199 & 199 & - & -- & - \\
\hline Yellow-poplar & 18,258 & 6,988 & 5,881 & 2,554 & 2,835 \\
\hline Persimmon & & $\ddot{-}$ & -- & $\ddot{-}$ & $-\bar{c}$ \\
\hline Sassafras & 1,334 & 412 & 531 & 105 & 286 \\
\hline Other hardwoods & 1,779 & 977 & 373 & -- & 429 \\
\hline Total & 308,751 & 70,771 & 130,525 & 21,825 & 85,630 \\
\hline All species & 314,041 & 75,024 & 131,562 & 21,825 & 85,630 \\
\hline
\end{tabular}


Table 71.--Current annual timber removals from growing stock and sawtimber on timberland by item and species group, Indiana, 1985

GROWING STOCK

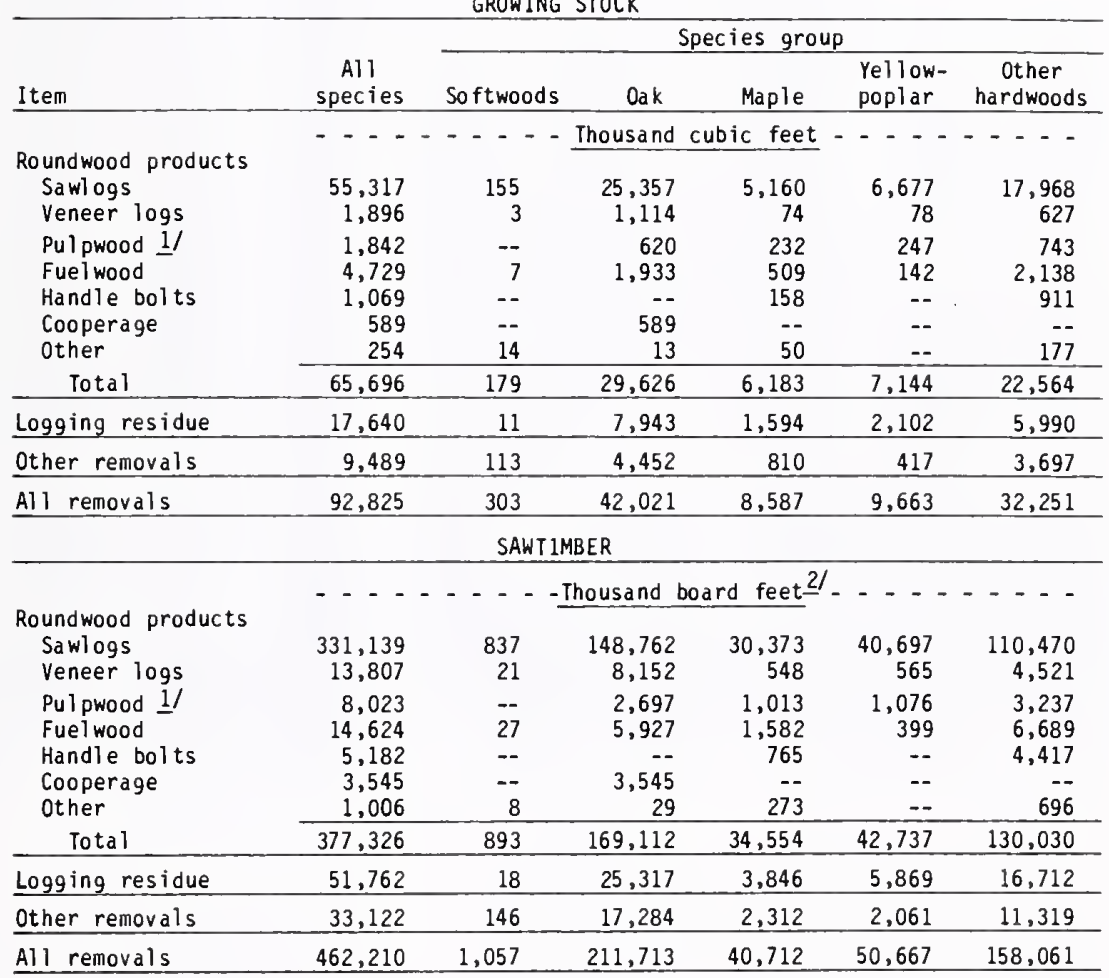

1/ Includes particle board and waferboard bolts.

2/ International $1 / 4$-inch rule. 
Table 72.-- Net annual growth and current annual timber removals from growing stock on timberland by species group and Forest Survey Unit, Indiana, 1985

(In thousand cubic feet)

\begin{tabular}{|c|c|c|c|c|c|c|c|c|c|c|}
\hline \multirow[b]{2}{*}{ Species group } & \multicolumn{5}{|c|}{ Growth } & \multicolumn{5}{|c|}{ Removals } \\
\hline & $\begin{array}{c}\text { All } \\
\text { Units }\end{array}$ & $\begin{array}{l}\text { Lower } \\
\text { Wabash } \\
\text { Unit }\end{array}$ & $\begin{array}{l}\text { Knobs } \\
\text { Unit }\end{array}$ & $\begin{array}{l}\text { Upland } \\
\text { Flats } \\
\text { Unit }\end{array}$ & $\begin{array}{c}\text { Northern } \\
\text { Unit }\end{array}$ & $\begin{array}{c}\text { All } \\
\text { Units }\end{array}$ & $\begin{array}{l}\text { Lower } \\
\text { Wabash } \\
\text { Unit }\end{array}$ & $\begin{array}{l}\text { Knobs } \\
\text { Unit }\end{array}$ & $\begin{array}{l}\text { Upland } \\
\text { Flats } \\
\text { Unit }\end{array}$ & $\begin{array}{c}\text { Nor thern } \\
\text { Unit }\end{array}$ \\
\hline \multicolumn{11}{|l|}{ So ftwoods } \\
\hline Pine & 3,838 & 932 & 2,331 & 179 & 396 & 184 & 2 & 151 & $1 /$ & 31 \\
\hline Baldcypress & 138 & 119 & 19 & -- & -- & -- & -- & -- & $=$ & -- \\
\hline Eastern redcedar & 2,133 & 57 & 1,382 & 677 & 17 & 119 & 1 & 101 & 17 & -- \\
\hline Other softwoods & 529 & -- & 281 & -- & 248 & -- & -- & -- & -- & -- \\
\hline Total & 6,638 & 1,108 & 4,013 & 856 & 661 & 303 & 3 & 252 & 17 & 31 \\
\hline \multicolumn{11}{|l|}{ Hardwoods } \\
\hline Select white oak & 15,350 & 2,735 & 8,369 & 1,479 & 2,767 & 12,310 & 2,804 & 5,191 & 796 & 3,519 \\
\hline other white oak & 2,425 & 83 & 2,334 & 1 & 7 & 2,700 & 162 & 2,306 & 12 & 220 \\
\hline Select red oak & 8,276 & 1,686 & 3,386 & 830 & 2,374 & 9,976 & 2,089 & 4,366 & 726 & 2,795 \\
\hline other red oak & 14,388 & 3,316 & 7,257 & 968 & 2,847 & 17,035 & 3,836 & 9,200 & 744 & 3,255 \\
\hline Select hickory & 5,583 & 1,354 & 1,937 & 640 & 1,652 & 3,397 & 1,101 & 1,320 & 106 & 870 \\
\hline other hickory & 6,402 & 1,548 & 2,511 & 952 & 1,391 & 3,625 & 1,142 & 1,800 & 162 & 521 \\
\hline Basswood & 1,693 & 250 & 124 & 197 & 1,122 & 935 & 147 & 156 & 66 & 566 \\
\hline Beech & 1,504 & 61 & 900 & 177 & 366 & 3,426 & 496 & 1,410 & 333 & 1,187 \\
\hline Hard maple & 13,833 & 2,249 & 7,127 & 1,816 & 2,641 & 5,437 & 882 & 2,646 & 203 & 1,706 \\
\hline Soft maple & 12,287 & 3,472 & 4,951 & 744 & 3,120 & 3.150 & 854 & 771 & 184 & 1,341 \\
\hline Elm & 4,654 & 452 & 362 & 581 & 3,259 & 888 & 260 & 247 & 10 & 371 \\
\hline Ash & 10,079 & 2,002 & 3,132 & 1,514 & 3,431 & 7,781 & 1,878 & 2,448 & 696 & 2,759 \\
\hline Sycamore & 6,232 & 1,658 & 2,688 & 653 & 1,233 & 3,242 & 815 & 1,079 & 212 & 1,136 \\
\hline Cottonwood & 3,789 & 972 & 478 & 99 & 2,240 & 1,920 & 508 & 304 & 160 & 948 \\
\hline Aspen & 728 & 52 & 517 & 120 & 39 & 285 & 24 & 65 & 15 & 181 \\
\hline Birch & 450 & 193 & 202 & - & 55 & 332 & 41 & 221 & 19 & 51 \\
\hline Sweetgum & 3,203 & 680 & 1,589 & 879 & 55 & 1,049 & 279 & 486 & 221 & 63 \\
\hline Tupelo & 1,134 & 295 & 603 & 228 & 8 & 538 & 143 & 282 & 88 & 25 \\
\hline Bl ack cherry & 2,961 & 511 & 944 & 325 & 1,181 & 1,519 & 321 & 476 & 86 & 636 \\
\hline Black walnut & 3,959 & 927 & 883 & 707 & 1,442 & 1,924 & 496 & 479 & 153 & 796 \\
\hline Yellow-poplar & 20,032 & 4,918 & 10,946 & 2,171 & 1,997 & 9,663 & 2,816 & 5,192 & 604 & 1,051 \\
\hline other hardwoods & 8,035 & 1,620 & 3,693 & 830 & 1,892 & 1,390 & 359 & 282 & 68 & 681 \\
\hline Total & 146,997 & 31,034 & 64,933 & 15,911 & 35,119 & 92,522 & 21,453 & 40,727 & 5,664 & 24,678 \\
\hline All species & 153,635 & 32,142 & 68,946 & 16,767 & 35,780 & 92,825 & 21,456 & 40,979 & 5,681 & 24,709 \\
\hline
\end{tabular}

1/Less than 500 cubic feet. 
Table 73... Net annual growth and current annual timber removals from sawtimber on timberland by species group and Forest Survey Unit, Indiana, 1985

(In thous and board feet) 1 '

\begin{tabular}{|c|c|c|c|c|c|c|c|c|c|c|}
\hline \multirow[b]{2}{*}{ Species group } & \multicolumn{5}{|c|}{ Growth } & \multicolumn{5}{|c|}{ Removals } \\
\hline & $\begin{array}{c}\text { All } \\
\text { Units }\end{array}$ & $\begin{array}{l}\text { Lower } \\
\text { Wabash } \\
\text { Unit }\end{array}$ & $\begin{array}{l}\text { Knobs } \\
\text { Unit }\end{array}$ & $\begin{array}{l}\text { Upland } \\
\text { Flats } \\
\text { Unit }\end{array}$ & $\begin{array}{c}\text { Northern } \\
\text { Unit }\end{array}$ & $\begin{array}{c}\text { Al1 } \\
\text { Units }\end{array}$ & $\begin{array}{l}\text { Lower } \\
\text { Wabash } \\
\text { Unit }\end{array}$ & $\begin{array}{l}\text { Knobs } \\
\text { Unit }\end{array}$ & $\begin{array}{l}\text { Upl and } \\
\text { Flats } \\
\text { Unit }\end{array}$ & $\begin{array}{c}\text { Northern } \\
\text { Unit }\end{array}$ \\
\hline $\begin{array}{l}\text { So ftwoods } \\
\text { Pine } \\
\text { 8aldc ypress } \\
\text { Eastern redcedar } \\
\text { Other softwoods }\end{array}$ & $\begin{array}{r}28,868 \\
744 \\
6,237 \\
3,454 \\
\end{array}$ & $\begin{array}{r}5,028 \\
643 \\
-72 \\
-- \\
\end{array}$ & $\begin{array}{r}18,343 \\
101 \\
6,090 \\
251 \\
\end{array}$ & $\begin{array}{r}501 \\
-- \\
212 \\
-- \\
\end{array}$ & $\begin{array}{r}4,996 \\
- \\
7 \\
3,203 \\
\end{array}$ & $\begin{array}{r}826 \\
-- \\
231 \\
-- \\
\end{array}$ & $\begin{array}{r}14 \\
-- \\
5 \\
-- \\
\end{array}$ & $\begin{array}{r}721 \\
-- \\
179 \\
-- \\
\end{array}$ & $\begin{array}{r}2 \\
-- \\
47 \\
--\end{array}$ & $\begin{array}{l}89 \\
-- \\
-- \\
--\end{array}$ \\
\hline Total & 39,303 & 5,599 & 24,785 & 713 & 8,206 & 1,057 & 19 & 900 & 49 & 89 \\
\hline $\begin{array}{l}\text { Hardwoods } \\
\text { Select white oak } \\
\text { Other white oak } \\
\text { Select red oak } \\
\text { Other red oak } \\
\text { Select hickory } \\
\text { Other hickory } \\
\text { Basswood } \\
\text { Beech } \\
\text { Hard maple } \\
\text { Soft maple } \\
\text { Elm } \\
\text { Ash } \\
\text { Sycamore } \\
\text { Cottonwood } \\
\text { Aspen } \\
8 \text { irch } \\
\text { Sweetgum } \\
\text { Tupelo } \\
\text { 8lack cherry } \\
\text { Black walnut } \\
\text { Yellow-poplar } \\
\text { Other hardwoods }\end{array}$ & $\begin{array}{r}79,020 \\
13,109 \\
50,361 \\
77,947 \\
28,196 \\
35,440 \\
9,019 \\
5,676 \\
59,429 \\
36,743 \\
6,401 \\
49,560 \\
31,449 \\
19,464 \\
5,517 \\
1,262 \\
11,577 \\
5,201 \\
16,699 \\
19,928 \\
90,476 \\
34,167\end{array}$ & $\begin{array}{r}12,302 \\
742 \\
8,458 \\
17,130 \\
9,622 \\
11,171 \\
2,651 \\
400 \\
14,850 \\
15,176 \\
53 \\
6,306 \\
7,065 \\
4,536 \\
-- \\
426 \\
1,331 \\
435 \\
1,724 \\
6,405 \\
19,746 \\
7,673 \\
\end{array}$ & $\begin{array}{r}48,329 \\
12,192 \\
17,443 \\
41,071 \\
8,735 \\
16,989 \\
795 \\
3,301 \\
32,476 \\
11,058 \\
1,246 \\
16,259 \\
15,371 \\
2,651 \\
4,602 \\
798 \\
6,916 \\
3,678 \\
8,857 \\
6,323 \\
54,629 \\
7,618 \\
\end{array}$ & $\begin{array}{r}5,639 \\
-24 \\
4,365 \\
3,870 \\
3,547 \\
3,185 \\
1,537 \\
402 \\
3,776 \\
1,882 \\
306 \\
6,756 \\
3,611 \\
395 \\
370 \\
-- \\
3,221 \\
1,031 \\
1,311 \\
3,021 \\
8,630 \\
4,816\end{array}$ & $\begin{array}{r}12,750 \\
199 \\
20,095 \\
15,876 \\
6,292 \\
4,095 \\
4,036 \\
1,573 \\
8,327 \\
8,627 \\
4,796 \\
20,239 \\
5,402 \\
11,882 \\
545 \\
38 \\
109 \\
57 \\
4,807 \\
4,179 \\
7,471 \\
14,060 \\
\end{array}$ & $\begin{array}{r}61,583 \\
12,740 \\
51,242 \\
86,148 \\
16,538 \\
17,140 \\
4,452 \\
17,612 \\
25,614 \\
15,098 \\
3,929 \\
36,840 \\
16,304 \\
11,072 \\
1,078 \\
1,679 \\
5,410 \\
2,748 \\
7,761 \\
11,003 \\
50,667 \\
4,495 \\
\end{array}$ & $\begin{array}{r}14,125 \\
695 \\
10,702 \\
19,477 \\
5,522 \\
5,633 \\
763 \\
2,581 \\
4,230 \\
4,171 \\
1,313 \\
9,105 \\
4,016 \\
2,949 \\
127 \\
227 \\
1,397 \\
711 \\
1,673 \\
2,993 \\
14,714 \\
1,012 \\
\end{array}$ & $\begin{array}{r}25,305 \\
10,908 \\
22,166 \\
47,087 \\
6,408 \\
8,213 \\
739 \\
7,195 \\
12,196 \\
3,736 \\
1,009 \\
11,284 \\
5,419 \\
1,660 \\
271 \\
1,085 \\
2,558 \\
1,436 \\
2,464 \\
2,865 \\
27,323 \\
995 \\
\end{array}$ & $\begin{array}{r}4,208 \\
47 \\
3,947 \\
3,798 \\
533 \\
788 \\
351 \\
1,700 \\
902 \\
921 \\
44 \\
3,295 \\
1,123 \\
943 \\
81 \\
99 \\
1,162 \\
462 \\
453 \\
987 \\
3,185 \\
240 \\
\end{array}$ & $\begin{array}{r}17,945 \\
1,090 \\
14,427 \\
15,786 \\
4,075 \\
2,506 \\
2,599 \\
6,136 \\
8,286 \\
6,270 \\
1,563 \\
13,156 \\
5,746 \\
5,520 \\
599 \\
268 \\
293 \\
139 \\
3,171 \\
4,158 \\
5,445 \\
2,248 \\
\end{array}$ \\
\hline Total & 686,641 & 148,202 & 321,337 & 61,647 & 155,455 & 461,153 & 108,136 & 202,322 & 29,269 & 121,426 \\
\hline All species & 725,944 & 153,801 & 346,122 & 62,360 & 163,661 & 462,210 & 108,155 & 203,222 & 29,318 & 121,515 \\
\hline
\end{tabular}

$1 /$ International $1 / 4$-inch rule. 

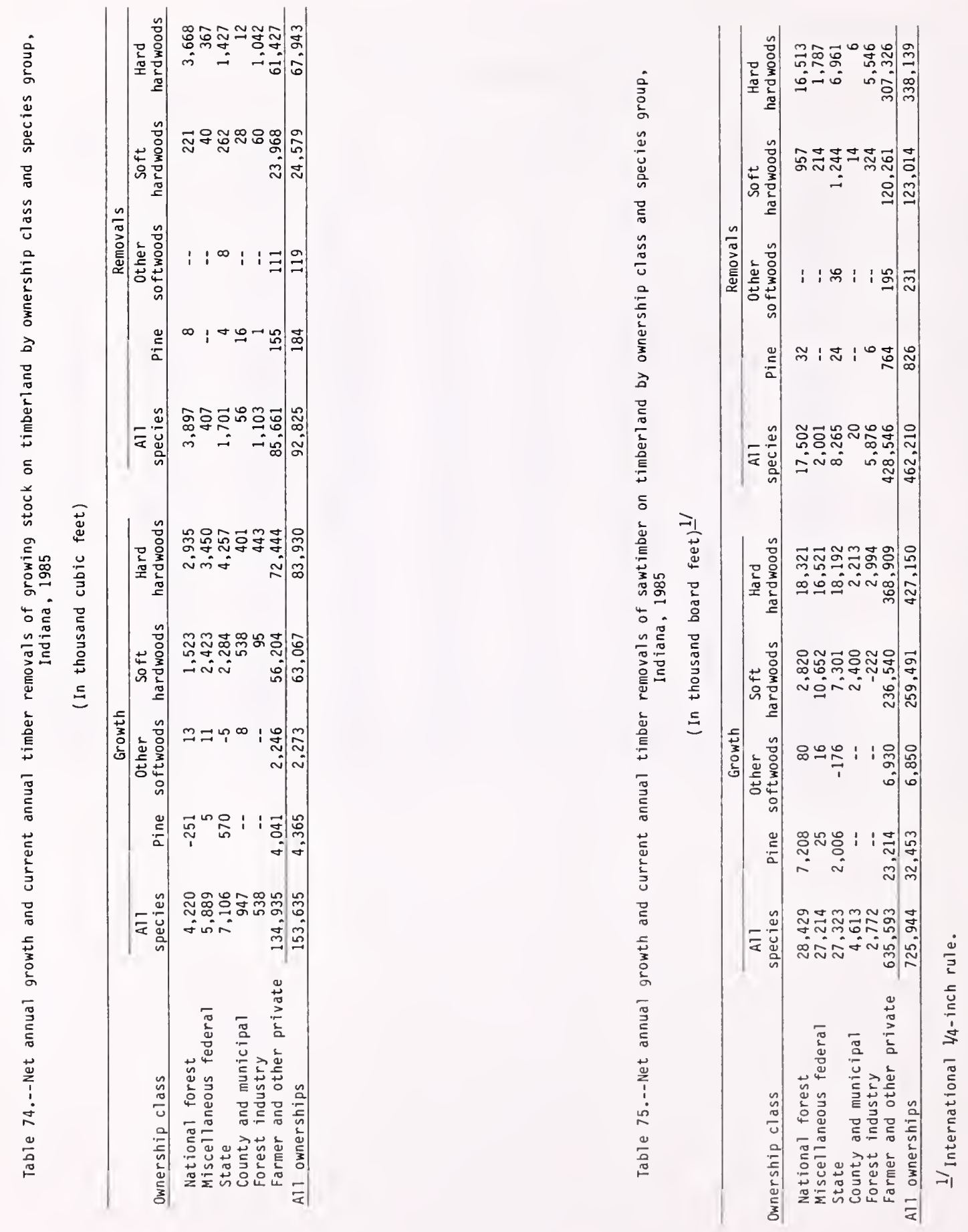
Table 76.--Annual mortality of growing stock and sawtimber on timberland by softwoods and hardwoods, Indiana, 1966 and 1985

\begin{tabular}{cccccc}
\hline & \multicolumn{2}{c}{ Growing stock } & & \multicolumn{2}{c}{ Sawt imber } \\
\cline { 2 - 3 } \cline { 5 - 6 } Species group & 1966 1/ & 1985 & & $1966-1 /$ & 1985 \\
\hline & \multicolumn{2}{c}{ Thousand cubic feet } & & \multicolumn{2}{c}{ Thousand board feet- } \\
\cline { 2 - 6 } Softwoods & 207 & 2,180 & & 176 & 4,532 \\
Hardwoods & 12,038 & 35,345 & & 37,090 & 96,717 \\
All species & 12,245 & 37,525 & & 37,266 & 101,249 \\
\hline
\end{tabular}

$1 /$ Figures have been adjusted from those published after the 1966 survey to conform to 1985 volumes because of changes in survey procedures.

2/ International $1 / 4$-inch rule.

Table 77.--Annual mortality of growing stock and sawtimber on timberland by species group, Indiana, 1985

\begin{tabular}{|c|c|c|}
\hline Species group & Growing stock & Sawtimber \\
\hline & Thousand & Thousand \\
\hline & cubic feet & board feet 1 \\
\hline \multicolumn{3}{|l|}{ Softwoods } \\
\hline Red pine & $\begin{array}{l}42 \\
16\end{array}$ & $\begin{array}{r}160 \\
34\end{array}$ \\
\hline White pine & 197 & 243 \\
\hline Shortleaf pine & 812 & 931 \\
\hline other yellow pines & 531 & 1,215 \\
\hline Tamarack & 46 & 249 \\
\hline Baldcypress & 156 & 703 \\
\hline Eastern redcedar & 329 & 988 \\
\hline Other softwoods & 51 & 9 \\
\hline Total & 2,180 & 4,532 \\
\hline \multicolumn{3}{|l|}{ Hardwoods } \\
\hline Select white oak & 1,548 & 5,140 \\
\hline Other white oak & 548 & 1,903 \\
\hline Select red oak & 1,465 & 5,737 \\
\hline Other red oak & 3,139 & 11,857 \\
\hline Select hickory & 1,264 & 3,866 \\
\hline Other hickory & 1,472 & 4,314 \\
\hline Basswood & 509 & 1,278 \\
\hline Beech & 515 & 1,945 \\
\hline Hard maple & 2,022 & 5,302 \\
\hline Soft maple & 2,237 & 6,757 \\
\hline Elm & 4,275 & 9,453 \\
\hline Ash & 2,195 & 5,561 \\
\hline Sycamore & 1,761 & 6,902 \\
\hline Cottonwood & 956 & 3,944 \\
\hline Willow & 581 & 2,088 \\
\hline Hackberry & 1,004 & 2,787 \\
\hline Aspen & 739 & 1,598 \\
\hline Birch & 94 & 45 \\
\hline Sweetgum & 615 & 1,929 \\
\hline Tupe10 & 228 & 814 \\
\hline Black cherry & 1,754 & 2,729 \\
\hline Black walnut & 1,146 & 1,964 \\
\hline Buttẹrnut & 135 & 454 \\
\hline Yellow-poplar & 1,005 & 4,264 \\
\hline Persimmon & 282 & 93 \\
\hline $\begin{array}{l}\text { Sassafras } \\
\text { Other hardwoods }\end{array}$ & $\begin{array}{l}1,994 \\
1,862\end{array}$ & $\begin{array}{l}1,702 \\
2,291\end{array}$ \\
\hline $\begin{array}{l}\text { Other hardwoods } \\
\text { Total }\end{array}$ & $\begin{array}{r}1,862 \\
35,345\end{array}$ & $\begin{array}{r}2,291 \\
96,717\end{array}$ \\
\hline $\begin{array}{ll}\text { Total } \\
\text { All species }\end{array}$ & 37,525 & 101,249 \\
\hline
\end{tabular}

$1 /$ International $1 / 4$-inch rule. 
Table 78.--Annual mortality of growing stock on timberland by species group and cause of death, Indiana, 1985

(In thousand cubic feet)

\begin{tabular}{|c|c|c|c|c|c|c|c|c|}
\hline \multirow[b]{2}{*}{ Species group } & \multirow[b]{2}{*}{$\begin{array}{c}\text { All } \\
\text { causes }\end{array}$} & \multicolumn{7}{|c|}{ Cause of death } \\
\hline & & Insects & Disease & Fire & Animals & Weather & Suppression & $\begin{array}{l}\text { Unknown } \\
\text { and other }\end{array}$ \\
\hline $\begin{array}{l}\text { Softwoods } \\
\text { Jack pine } \\
\text { Red pine } \\
\text { White pine } \\
\text { Shortleaf pine } \\
\text { Other yellow pines } \\
\text { Tamarack } \\
\text { Baldcypress } \\
\text { Eastern redcedar } \\
\text { Other softwoods }\end{array}$ & $\begin{array}{r}42 \\
16 \\
197 \\
812 \\
531 \\
46 \\
156 \\
329 \\
51 \\
\end{array}$ & $\begin{array}{l}-- \\
-- \\
-- \\
-- \\
-- \\
-- \\
-- \\
-- \\
--\end{array}$ & $\begin{array}{c}-- \\
-- \\
8 \\
-- \\
-- \\
-- \\
-- \\
-- \\
--\end{array}$ & $\begin{array}{l}-- \\
-- \\
-- \\
-- \\
15 \\
-- \\
-- \\
-- \\
--\end{array}$ & $\begin{array}{l}-- \\
-- \\
-- \\
-- \\
-- \\
-- \\
-- \\
-- \\
--\end{array}$ & $\begin{array}{r}-- \\
-- \\
-- \\
25 \\
173 \\
-- \\
-- \\
-- \\
--\end{array}$ & $\begin{array}{r}-- \\
-- \\
19 \\
57 \\
-- \\
-- \\
-\because \\
3 \\
--\end{array}$ & $\begin{array}{r}42 \\
16 \\
170 \\
730 \\
343 \\
46 \\
156 \\
326 \\
51\end{array}$ \\
\hline Total & 2,180 & -- & 8 & 15 & -- & 198 & 79 & 1,880 \\
\hline $\begin{array}{l}\text { Hardwoods } \\
\text { Select white oak } \\
\text { other white oak } \\
\text { Select red oak } \\
\text { Other red oak } \\
\text { Select hickory } \\
\text { Other hickory } \\
\text { Basswood } \\
\text { Beech } \\
\text { Hard maple } \\
\text { Soft maple } \\
\text { Elm } \\
\text { Ash } \\
\text { Sycamore } \\
\text { Cottonwood } \\
\text { Willow } \\
\text { Hackberry } \\
\text { Aspen } \\
\text { Birch } \\
\text { Sweetgum } \\
\text { Tupelo } \\
\text { Black cherry } \\
\text { Black walnut } \\
\text { Butternut } \\
\text { Yellow-poplar } \\
\text { Persimmon } \\
\text { Sassafras } \\
\text { Other hardwoods }\end{array}$ & $\begin{array}{r}1,548 \\
548 \\
1,465 \\
3,139 \\
1,264 \\
1,472 \\
509 \\
515 \\
2,022 \\
2,237 \\
4,275 \\
2,195 \\
1,761 \\
956 \\
581 \\
1,004 \\
739 \\
94 \\
615 \\
228 \\
1,754 \\
1,146 \\
135 \\
1,005 \\
282 \\
1,994 \\
1,862 \\
\end{array}$ & $\begin{array}{r}1 \\
-- \\
-- \\
2 \\
-- \\
10 \\
-- \\
-- \\
-- \\
8 \\
15 \\
-- \\
-- \\
-- \\
-- \\
-- \\
-- \\
-- \\
-- \\
-- \\
70 \\
2 \\
-- \\
3 \\
-- \\
6 \\
-- \\
\end{array}$ & $\begin{array}{r}86 \\
83 \\
116 \\
292 \\
22 \\
111 \\
46 \\
1 \\
58 \\
101 \\
134 \\
74 \\
78 \\
6 \\
8 \\
77 \\
100 \\
-- \\
4 \\
18 \\
77 \\
108 \\
-- \\
34 \\
-- \\
232 \\
118 \\
\end{array}$ & $\begin{array}{l}-- \\
13 \\
57 \\
50 \\
-- \\
-- \\
-- \\
-- \\
-- \\
-- \\
-- \\
39 \\
-- \\
-- \\
-- \\
-- \\
-- \\
-- \\
-- \\
-- \\
-- \\
-- \\
-- \\
-- \\
-- \\
-- \\
-- \\
\end{array}$ & $\begin{array}{r}-- \\
-- \\
10 \\
-- \\
-- \\
-- \\
-- \\
-- \\
2 \\
-- \\
22 \\
4 \\
-- \\
-- \\
-- \\
-- \\
-- \\
-- \\
-- \\
-- \\
-- \\
-- \\
-- \\
-- \\
-- \\
-- \\
-- \\
\end{array}$ & $\begin{array}{r}11 \\
77 \\
74 \\
331 \\
60 \\
70 \\
162 \\
53 \\
131 \\
160 \\
141 \\
113 \\
137 \\
231 \\
30 \\
51 \\
31 \\
-- \\
27 \\
-- \\
28 \\
2 \\
10 \\
217 \\
-- \\
-- \\
14 \\
\end{array}$ & $\begin{array}{r}25 \\
-- \\
-- \\
-- \\
1 \\
1 \\
-- \\
9 \\
-- \\
25 \\
29 \\
19 \\
2 \\
-- \\
-- \\
87 \\
-- \\
-- \\
6 \\
-- \\
77 \\
54 \\
-- \\
-- \\
-- \\
69 \\
24 \\
\end{array}$ & $\begin{array}{r}1,425 \\
375 \\
1,208 \\
2,464 \\
1,181 \\
1,280 \\
301 \\
452 \\
1,831 \\
1,943 \\
3,934 \\
1,946 \\
1,544 \\
719 \\
543 \\
789 \\
608 \\
94 \\
578 \\
210 \\
1,502 \\
980 \\
125 \\
751 \\
282 \\
1,687 \\
1,706 \\
\end{array}$ \\
\hline Tota] & 35,345 & 117 & 1,984 & 159 & 38 & 2,161 & 428 & 30,458 \\
\hline All species & 37,525 & 117 & 1,992 & 174 & 38 & 2,359 & 507 & 32,338 \\
\hline
\end{tabular}


Table 79.--Annual mortality of sawtimber on timberland by species group and cause of death, Indiana, 1985 (In thousand board feet) 1 /

\begin{tabular}{|c|c|c|c|c|c|c|c|c|}
\hline \multirow[b]{2}{*}{ Species group } & \multirow[b]{2}{*}{$\begin{array}{c}\text { Al1 } \\
\text { causes }\end{array}$} & \multicolumn{7}{|c|}{ Cause of death } \\
\hline & & Insects & Disease & Fire & Animals & Weather & Suppression & $\begin{array}{l}\text { Unknown } \\
\text { and other }\end{array}$ \\
\hline \multicolumn{9}{|l|}{ Softwoods } \\
\hline Jack pine & 160 & -- & -- & -- & -- & -- & -- & 160 \\
\hline Red pine & 34 & -- & -- & -- & -- & -- & -- & 34 \\
\hline White pine & 243 & -- & -- & - & -- & 61 & - & 182 \\
\hline Shortleaf pine & 931 & - & - & -- & - & -- & -- & 931 \\
\hline other yellow pines & 1,215 & -- & -- & -- & -- & 486 & -- & 729 \\
\hline Tamarack & 249 & -- & -- & -- & -- & -- & -- & 249 \\
\hline 8aldcypress & 703 & -- & -- & -- & -- & - & -- & 703 \\
\hline Eastern redcedar & 988 & -- & - & -- & -- & -- & -. & 988 \\
\hline Other softwoods & 9 & -- & -- & -- & -- & -- & -- & 9 \\
\hline Total & 4,532 & -- & -- & -- & -- & 547 & -- & 3,985 \\
\hline \multicolumn{9}{|l|}{ Hardwoods } \\
\hline Select white oak & 5,140 & 20 & 500 & -- & 4 & 2 & 4 & 4,610 \\
\hline Other white oak & 1,903 & -- & 474 & - & -- & 384 & - & 1,045 \\
\hline Select red oak & 5,737 & -- & 469 & 299 & -- & 357 & -- & 4,612 \\
\hline other red oak & 11,857 & 3 & 1,098 & 186 & -- & 1,433 & -- & 9,137 \\
\hline Select hickory & 3,866 & - & 10 & - & -- & 296 & -- & 3,560 \\
\hline other hickory & 4,314 & -- & 260 & -- & -- & 402 & -- & 3,652 \\
\hline Basswood & 1,278 & -- & 170 & -- & -- & 130 & -- & 978 \\
\hline Beech & 1,945 & -- & 7 & - & - & 198 & 41 & 1,699 \\
\hline Hard maple & 5,302 & $\ldots$ & 213 & $\ldots$ & 5 & 322 & -2 & 4,762 \\
\hline So ft maple & 6,757 & 35 & 396 & -- & -- & 420 & -- & 5,906 \\
\hline Elm & 9,453 & 21 & 294 & -. & 49 & 480 & 42 & 8,567 \\
\hline Ash & 5,561 & -- & 229 & 115 & 6 & 449 & - & 4,762 \\
\hline Sycamore & 6,902 & -- & 397 & -- & -- & 673 & -- & 5,832 \\
\hline Cottonwood & 3,944 & -- & 27 & -- & -- & 1,039 & -- & 2,878 \\
\hline Willow & 2,088 & -- & - & -- & -- & 142 & -- & 1,946 \\
\hline Hackberry & 2,787 & -- & 151 & - & - & 257 & -- & 2,379 \\
\hline Aspen & 1,598 & - & - & -- & -- & -- & -- & 1,598 \\
\hline Birch & 45 & -- & -- & -- & -- & -- & -- & 45 \\
\hline Sweetgum & 1,929 & .. & 25 & -. & -- & 165 & - & 1,739 \\
\hline Tupelo & 814 & -- & 91 & -- & -- & - & -. & 723 \\
\hline Black cherry & 2,729 & 231 & 43 & -- & -- & 119 & 256 & 2,080 \\
\hline Black walnut & 1,964 & 11 & 14 & -- & -- & 20 & - & 1,919 \\
\hline Butternut & 454 & -- & $\rightarrow$ & -- & -- & 52 & -- & 402 \\
\hline Yellow-poplar & 4,264 & 12 & 202 & -- & -- & 1,241 & -- & 2,809 \\
\hline Persimmon & 93 & $\ldots$ & -. & -- & -- & - & -- & 93 \\
\hline Sassafras & 1,702 & 20 & 155 & -- & -- & -- & -- & 1,527 \\
\hline Other hardwoods & 2,291 & -- & 16 & -- & -- & 33 & -- & 2,242 \\
\hline Total & 96,717 & 353 & 5,241 & 600 & 64 & 8,614 & 343 & 81,502 \\
\hline A11 species & 101,249 & 353 & 5,241 & 600 & 64 & 9,161 & 343 & 85,487 \\
\hline
\end{tabular}

$1 /$ International $1 / 4$-inch rule.

Table 80.--Annual mortality of growing stock and sawtimber on timberland by ownership class and species group, Indiana, 1985

\begin{tabular}{|c|c|c|c|c|c|c|}
\hline \multirow[b]{2}{*}{ Ownership class } & \multicolumn{3}{|c|}{ Growing stock } & \multicolumn{3}{|c|}{ Sawt imber } \\
\hline & $\begin{array}{c}\text { All } \\
\text { species }\end{array}$ & Softwoods & Hardwoods & $\begin{array}{c}\text { All } \\
\text { species }\end{array}$ & Softwoods & Hardwoods \\
\hline & --- & sand cubic & et - - - & -- & sand board & $=1 / \ldots$ \\
\hline $\begin{array}{l}\text { National forest } \\
\text { Miscellaneous federal } \\
\text { State } \\
\text { County and municipal } \\
\text { Forest industry } \\
\text { Farmer } \\
\text { Misc. private-corp. } \\
\text { Misc. private-indiv. }\end{array}$ & $\begin{array}{r}1,769 \\
1,368 \\
1,903 \\
418 \\
194 \\
14,241 \\
3,828 \\
13,804 \\
\end{array}$ & $\begin{array}{r}693 \\
21 \\
279 \\
-- \\
-- \\
263 \\
201 \\
723 \\
\end{array}$ & $\begin{array}{r}1,076 \\
1,347 \\
1,624 \\
418 \\
194 \\
13,978 \\
3,627 \\
13,081 \\
\end{array}$ & $\begin{array}{r}3,241 \\
3,909 \\
5,552 \\
1,221 \\
833 \\
38,833 \\
10,846 \\
36,814 \\
\end{array}$ & $\begin{array}{r}766 \\
5 \\
823 \\
-. \\
-- \\
501 \\
443 \\
1,994 \\
\end{array}$ & $\begin{array}{r}2,475 \\
3,904 \\
4,729 \\
1,221 \\
833 \\
38,332 \\
10,403 \\
34,820 \\
\end{array}$ \\
\hline All owners & 37,525 & 2,180 & 35,345 & 101,249 & 4,532 & 96,717 \\
\hline
\end{tabular}

1/ International $1 / 4$-inch rule. 


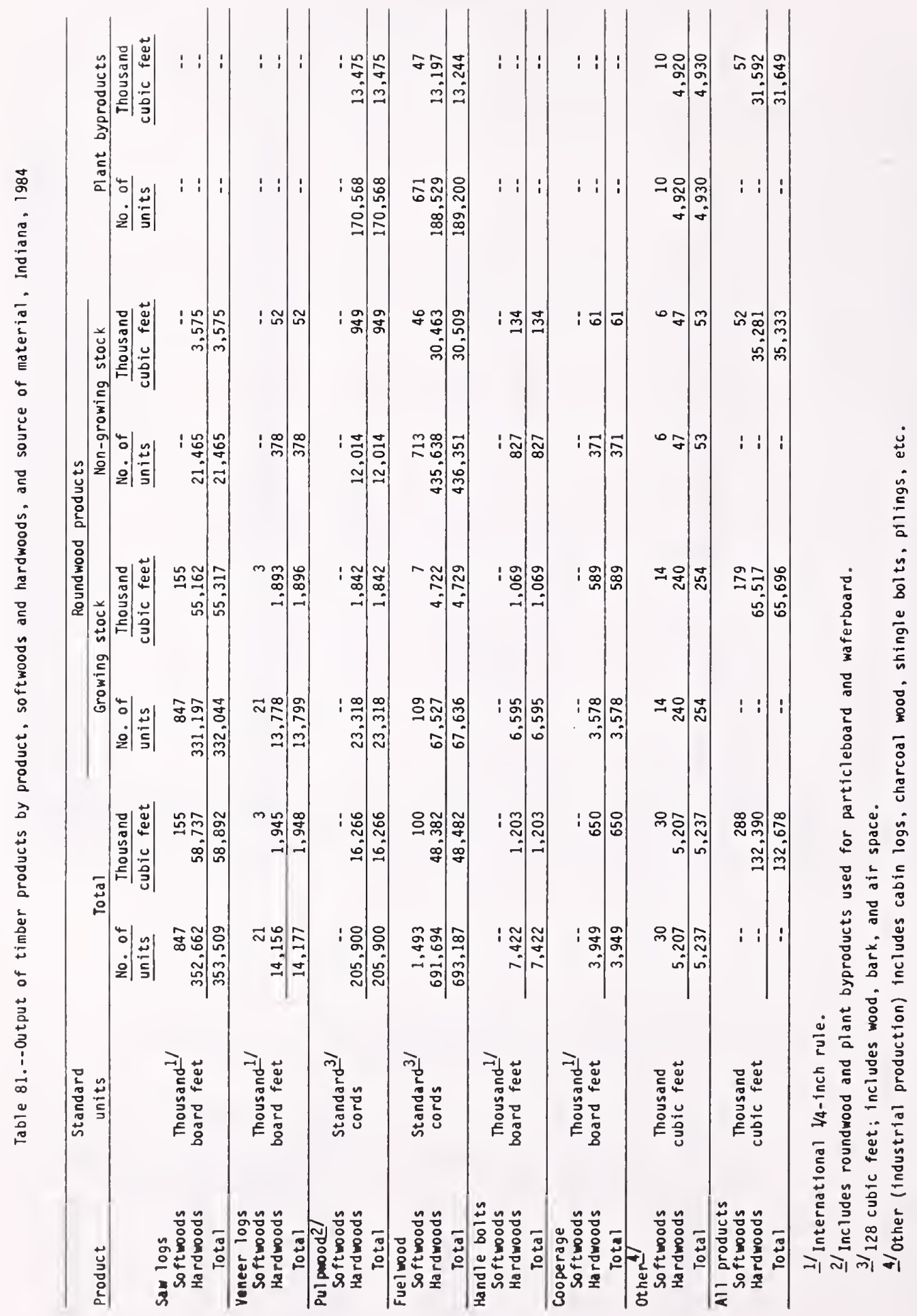


Table 82.--0utput of roundwood products by product softwoods and hardwoods, and source of material, Indiana, 1984

(In thousand cubic feet)

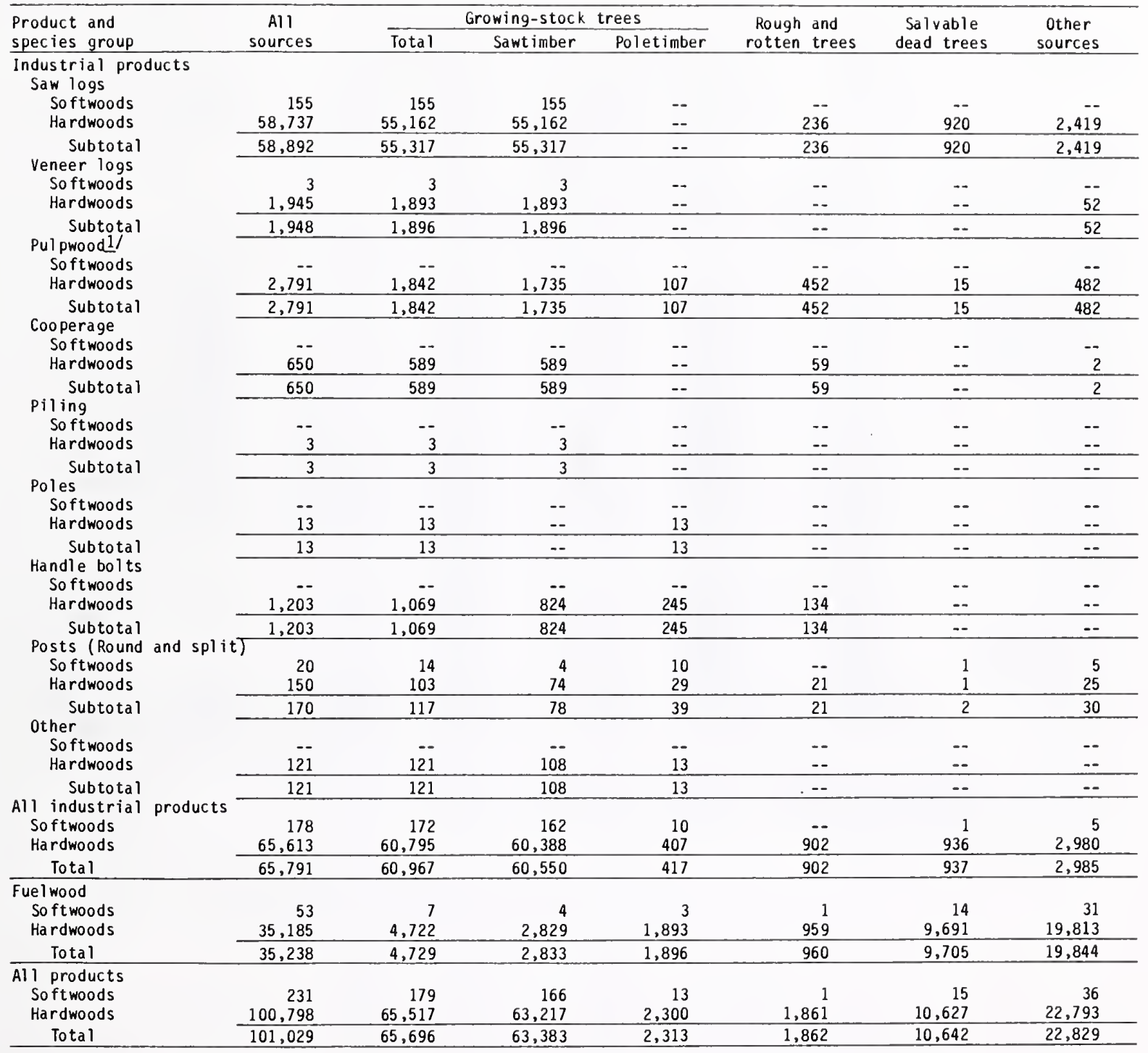

1/ Includes particleboard and waferboard bolts. 
Table 83.--Timber products from roundwood by species group and product, Indiana, 1984

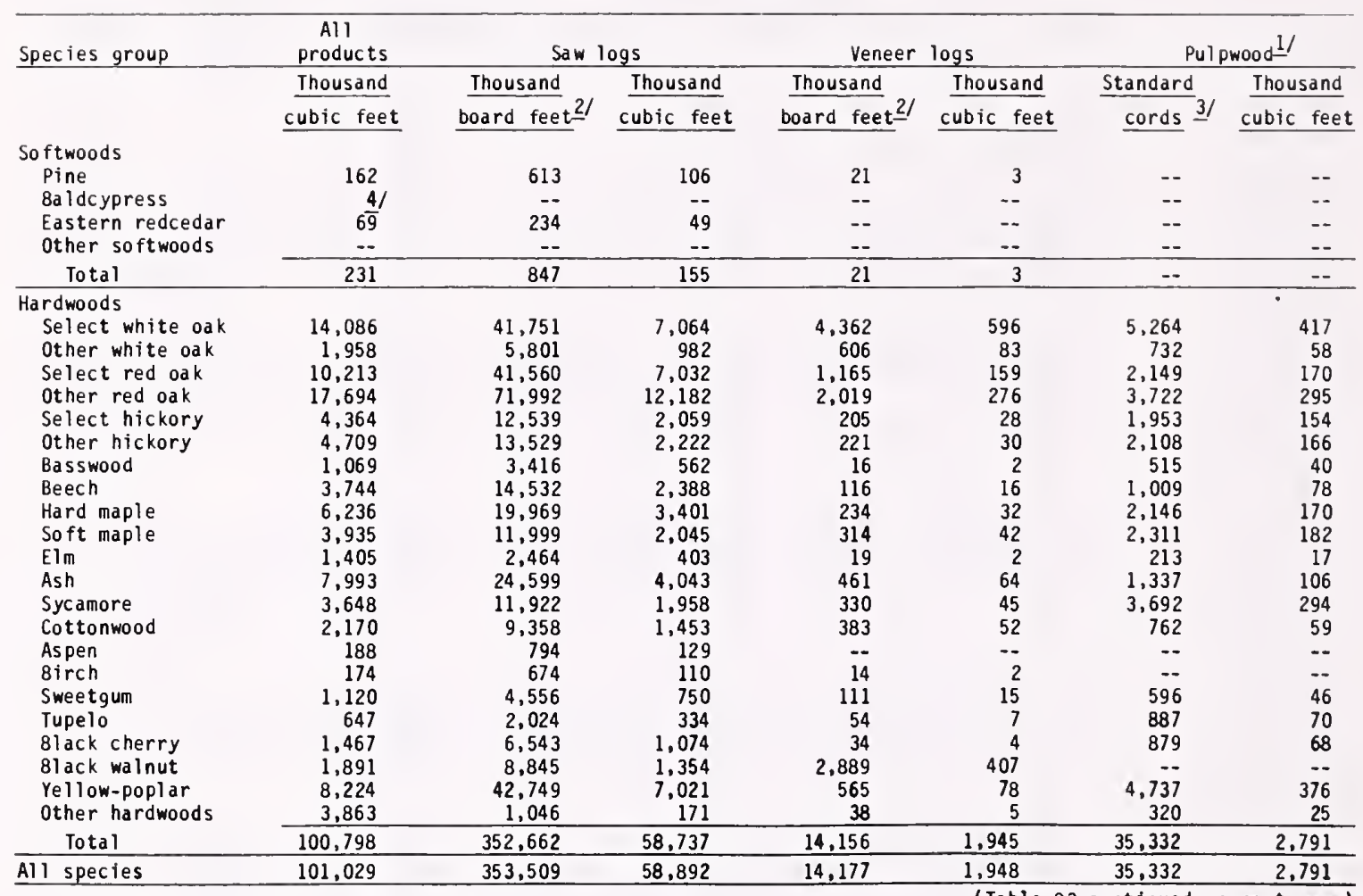

$1 /$ Includes particleboard and waferboard bolts.

2/ International $4 / 4$-inch rule.

$3 / 128$ cubic feet; includes wood, bark, and alr space.

4/Less than 500 cubic feet. 
(Table 83 continued)

\begin{tabular}{|c|c|c|c|c|c|c|c|}
\hline \multirow[t]{3}{*}{ Species group } & \multicolumn{2}{|c|}{ Fuel wood } & \multicolumn{2}{|c|}{ Handle Bolts } & \multicolumn{2}{|c|}{ Cooperage } & \multirow{2}{*}{$\begin{array}{c}\begin{array}{c}\text { Other } \\
\text { products }\end{array} \\
\text { Thousand }\end{array}$} \\
\hline & \multirow{2}{*}{$\frac{\text { Standard }}{\text { cords }} 3 /$} & Thous and & Thousand & Thousand & Thousand & Thousand & \\
\hline & & cubic feet & board feet $2 /$ & cubic feet & board feet $-2 /$ & cubic feet & cubic feet \\
\hline \multicolumn{8}{|l|}{ So ftwoods } \\
\hline Pine & 782 & 50 & - & -- & -- & -- & 3 \\
\hline Ba ldcypress & - & - & -- & -- & - & -- & $4 /$ \\
\hline Eastern redcedar & 40 & 3 & -- & -- & -- & -- & $\overline{17}$ \\
\hline Other softwoods & -- & -- & -- & -- & -- & -- & -- \\
\hline Total & 822 & 53 & -- & 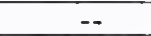 & 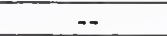 & -- & 20 \\
\hline Hardwoods & & & & & & $\cdot$ & \\
\hline Select white oak & 77,609 & 5,432 & - & -- & 3,467 & 571 & 6 \\
\hline other white oak & 10,784 & 755 & -- & - & 482 & 79 & 1 \\
\hline Select red oak & 40,705 & 2,850 & -- & - & -- & -- & 2 \\
\hline other red oak & 70,512 & 4,937 & -- & -- & - & -. & 4 \\
\hline Select hickory & 29,984 & 2,096 & 156 & 25 & -- & -- & 2 \\
\hline other hickory & 32,353 & 2,261 & 169 & 27 & -- & -- & 3 \\
\hline Basswood & 6,659 & 465 & -- & -- & -- & -- & - \\
\hline Beech & 18,007 & 1,262 & - & - & - & - & - \\
\hline Hard maple & 35,110 & 2,455 & 1,095 & 178 & -- & - & -. \\
\hline Soft maple & 23,106 & 1,616 & 1,00 & -- & -- & - & 50 \\
\hline Elm & 14,091 & 983 & -- & - & - & - & - \\
\hline Ash & 40,039 & 2,801 & 6,002 & 973 & - & - & 6 \\
\hline Sycamore & 19,236 & 1,345 & 0,00 & -2 & - & - & 6 \\
\hline Cottonwood & 7,938 & 553 & -- & -- & -- & -- & 53 \\
\hline Aspen & 907 & 59 & - & - & -- & -- & - \\
\hline Birch & 873 & 56 & -- & -- & -- & -- & 6 \\
\hline Sweetgum & 4,459 & 308 & - & - & - & -- & 1 \\
\hline Tupelo & 3,370 & 236 & -- & -- & -- & -- & -- \\
\hline Black cherry & 4,618 & 321 & -- & -- & -- & -- & -- \\
\hline Black walnut & 1,875 & 130 & $-\infty$ & -- & - & - & -- \\
\hline Yellow-poplar & 10,670 & 749 & -- & -- & -- & - & -- \\
\hline other hardwoods & 50,260 & 3,515 & -- & - & -- & -- & 147 \\
\hline Total & 503,165 & 35,185 & 7,422 & 1,203 & 3,949 & 650 & 287 \\
\hline All species & 503,987 & 35,238 & 7,422 & 1,203 & 3,949 & 650 & 307 \\
\hline
\end{tabular}

2/ International $1 / 4$-inch rule.

3/ 128 cubic feet; includes wood, bark, and air space.

$4 /$ Less than 500 cubic feet. 
Table 84.--Volume of primary plant residue by use and type of residue, Indiana, 1984

(In thousand cubic feet)

\begin{tabular}{|c|c|c|c|c|c|c|c|c|}
\hline \multirow[b]{3}{*}{ Use } & \multicolumn{6}{|c|}{ Wood residue } & & \\
\hline & \multicolumn{2}{|c|}{ Total } & \multicolumn{2}{|c|}{ Coarse $1 /$} & \multicolumn{2}{|c|}{ Fine 21} & \multicolumn{2}{|c|}{ Bark } \\
\hline & So ftwoods & Hardwoods & So ftwoods & Hardwoods & So ftwoods & Hardwoods & So f twoods & Hardwoods \\
\hline Fiber products ${ }^{3 /}$ & 7.7 & $11,983.1$ & 5.8 & $11,526.9$ & 1.9 & 456.2 & -. & 54.3 \\
\hline Charcoal & -- & 19.4 & -- & -- & -- & 19.4 & -- & -- \\
\hline Industrial fuel & 28.2 & $7,013.0$ & 11.0 & $1,326.7$ & 17.2 & $5,686.3$ & 13.9 & $4,287.8$ \\
\hline Domestic fuel & 19.2 & $6,183.9$ & 19.2 & $5,821.5$ & -- & 362.4 & 10.0 & $3,275.9$ \\
\hline Miscellaneous $4 /$ & 9.7 & $4,901.2$ & -- & 320.2 & 9.7 & $4,581.0$ & 0.1 & $4,902.9$ \\
\hline Not used $5 /$ & 24.0 & $2,980.3$ & 14.7 & $1,114.0$ & 9.3 & $1,866.3$ & 9.9 & $1,354.8$ \\
\hline Total & 88.8 & $33,080.9$ & 50.7 & $20,109.3$ & 38.1 & $12,971.6$ & 33.9 & $13,875.7$ \\
\hline
\end{tabular}

1/Suitable for chipping such as slabs, edgings, veneer cores, etc.

2/ Not suitable for chipping such as sawdust, veneer clippings, etc.

$3 /$ For manufacture of pulp, hardboard, or roofing felt.

4/Livestock bedding, mulch, small dimension, and specialty items.

5/Includes residue burned as waste. 


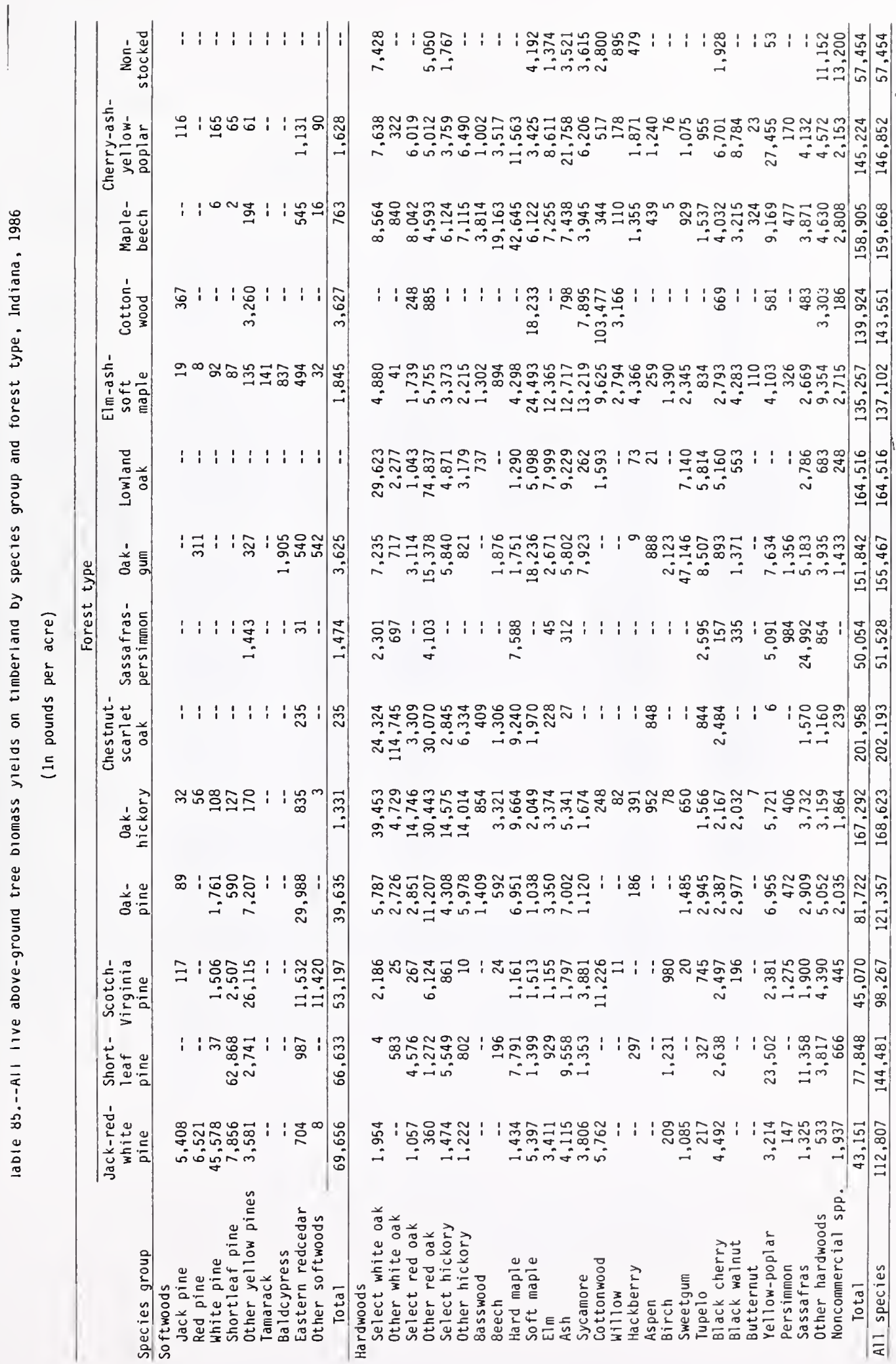


Table $\overline{86 .--A l l}$ live tree biomass on timberland by species group and forest type, Indiana, 1986

(In green tons)

\begin{tabular}{|c|c|c|c|c|c|c|c|c|}
\hline \multirow[b]{2}{*}{ Species group } & \multirow[b]{2}{*}{$\begin{array}{c}\text { All } \\
\text { types }\end{array}$} & \multicolumn{7}{|c|}{ Forest type } \\
\hline & & $\begin{array}{l}\text { Jack-red- } \\
\text { white } \\
\text { pine }\end{array}$ & $\begin{array}{c}\text { Shortleaf } \\
\text { pine }\end{array}$ & $\begin{array}{l}\text { Scotch- } \\
\text { Virginia } \\
\text { pine }\end{array}$ & $\begin{array}{l}\text { Oak- } \\
\text { pine }\end{array}$ & $\begin{array}{c}\text { Oak- } \\
\text { hickory }\end{array}$ & $\begin{array}{c}\text { Chestnut- } \\
\text { scarlet } \\
\text { oak }\end{array}$ & $\begin{array}{l}\text { Sassa fras - } \\
\text { pers immon }\end{array}$ \\
\hline \multicolumn{9}{|l|}{ Softwoods } \\
\hline Jack pine & 227,313 & 147,906 & -- & 4,131 & 4,624 & 21,825 & -- & -- \\
\hline Red pine & 228,049 & 178,336 & -- & & & 38,171 & -- & -- \\
\hline White pine & $1,560,109$ & $1,246,546$ & 447 & 53,160 & 91,734 & 73,682 & -- & -- \\
\hline Shortleaf pine & $1,230,559$ & 214,856 & 751,276 & 88,511 & 30,745 & 86,712 & -- & -- \\
\hline other yellow pines & $1,768,840$ & 97,946 & 32,749 & 921,862 & 375,489 & 116,261 & -- & 14,290 \\
\hline Tamarack & 58,668 & & - & & & & -- & -- \\
\hline Baldcypress & 396,597 & & -- & -- & -- & -- & -- & -- \\
\hline Eastern redcedar & $3,433,201$ & 19,250 & 11,800 & 407,084 & $1,562,383$ & 572,359 & 5,410 & 306 \\
\hline 0ther softwoods & 469,673 & 210 & -- & 403,140 & -- & 1,954 & -- & - \\
\hline Total & $9,373,009$ & $1,905,050$ & 96,272 & 877,888 & $.064,975$ & 910,964 & 5,410 & 14,596 \\
\hline \multicolumn{9}{|l|}{ Hardwoods } \\
\hline Select white oak & $37,573,334$ & 53,455 & 44 & 77,178 & 301,520 & $27,040,923$ & 560,659 & 22,778 \\
\hline $\begin{array}{l}\text { Other whit } \\
\text { Select red }\end{array}$ & $6,631,031$ & & 6,966 & 899 & 142,008 & $3,241,047$ & $2,644,865$ & 6,902 \\
\hline Select $r$ & .051 & 28,900 & 54,687 & 9,442 & 148,526 & $10,106,626$ & 76,270 & \\
\hline other red oak & 402 & 9,841 & 15,205 & 216,165 & 583,871 & $20,865,569$ & 693,110 & 40,619 \\
\hline Select hicl & 212 & 40,323 & 66,311 & 30,405 & 224,424 & 89,676 & 65,576 & -- \\
\hline other hickory & 16 & 33,410 & 9,580 & 368 & 311,438 & $9,604,995$ & 146,007 & -- \\
\hline Basswood & $3,423,210$ & -- & & -. & 73,427 & 585,298 & 9,420 & -- \\
\hline Beech & 226 & -- & 2,339 & 832 & 30,860 & $2,276,099$ & 30,106 & -- \\
\hline Hard maple & 942 & 39,207 & 93,103 & 40,968 & 362,165 & 23,953 & 212,992 & 75,119 \\
\hline Soft maple & $16,820,474$ & 147,621 & 16,723 & 53,396 & 54,057 & $1,404,695$ & 45,397 & - \\
\hline $\mathrm{Elm}$ & $14,359,453$ & 93,283 & 11,097 & 40,785 & 174,525 & $2,312,744$ & 5,263 & 448 \\
\hline Ash & 753 & 112,545 & 114,214 & 63,423 & 64,816 & 100 & 621 & 3,091 \\
\hline Sycamore & 62,692 & 104,089 & 16,173 & 137,002 & 58,345 & $1,147,523$ & - & - \\
\hline Cottonwood & 6 & 157,591 & -- & 396,268 & . & 16 & $\ldots$ & -- \\
\hline Willor & .575 & -- & -- & 392 & -- & 55,890 & -- & -- \\
\hline Hackberry & 政 & -. & 3,551 & -- & 9,667 & 268,159 & -- & -- \\
\hline Aspen & 421,627 & -- & -- & -. & -- & 652,541 & 19,542 & -- \\
\hline Birch & $76766 ?$ & 5,710 & 14,710 & 34,591 & -- & 53,571 & -. & -- \\
\hline Sweetgum & $3,662,670$ & 29,671 & -- & 713 & 77,358 & 445,620 & -- & -- \\
\hline Tupelo & $3,030,861$ & 5,946 & 3,905 & 26,293 & 153,454 & $1,073,205$ & 19,459 & 25,692 \\
\hline Black cherry & $7,378,323$ & 122,861 & 31,519 & 88,150 & 124,379 & $1,485,236$ & 57,262 & 1,558 \\
\hline Black walnut & $7,813,803$ & -- & -- & 6,924 & 155,119 & $1,392,820$ & -- & 3,319 \\
\hline But ternut & 217,724 & -. & -- & -- & & 5,099 & -. & \\
\hline Yellow-poplar & $20,117,950$ & 87,912 & 280,848 & 84,051 & 362,366 & $3,921,082$ & 141 & 50,397 \\
\hline Persimmon & 822,557 & 4,024 & -- & 44,990 & 24,577 & 278,454 & - & 9,742 \\
\hline Sassafras & $7,768,847$ & 36,239 & 135,723 & 67,074 & 151,567 & $2,557,795$ & 36,180 & 247,423 \\
\hline Other hardwoods & $10,694,649$ & 14,586 & 45,619 & 154,955 & 263,217 & $2,165,018$ & 26,747 & 8,454 \\
\hline \multirow{2}{*}{$\begin{array}{l}\text { Noncommercial spp. } \\
\text { Total }\end{array}$} & $4,984,080$ & 52,990 & 7,961 & 15,691 & 106,028 & $1,277,553$ & 5,509 & -- \\
\hline & $318,217,249$ & $1,180,204$ & 930,278 & $1,590,955$ & $4,257,714$ & $114,662,174$ & $4,655,126$ & 495,542 \\
\hline All species & $327,590,258$ & $3,085,254$ & $1,726,550$ & $3,468,843$ & $6,322,689$ & $115,573,138$ & $4,660,536$ & 510,138 \\
\hline
\end{tabular}


(Table 86 continued)

\begin{tabular}{|c|c|c|c|c|c|c|c|}
\hline \multirow[b]{2}{*}{ Species group } & \multicolumn{7}{|c|}{ Forest type } \\
\hline & $\begin{array}{l}\text { Oak- } \\
\text { gum }\end{array}$ & $\begin{array}{c}\text { Lowl and } \\
\text { oak }\end{array}$ & $\begin{array}{l}\text { Elm-ash- } \\
\text { soft maple }\end{array}$ & $\begin{array}{l}\text { Cotton- } \\
\text { wood }\end{array}$ & $\begin{array}{l}\text { Maple- } \\
\text { beech }\end{array}$ & $\begin{array}{c}\text { Cherry-ash- } \\
\text { yellow- } \\
\text { poplar }\end{array}$ & $\begin{array}{c}\text { Non- } \\
\text { stocked }\end{array}$ \\
\hline $\begin{array}{l}\text { Softwoods } \\
\text { Jack pine } \\
\text { Red pine } \\
\text { White pine } \\
\text { Shortleaf pine } \\
\text { Other yellow pines } \\
\text { Tamarack } \\
\text { 8aldcypress } \\
\text { Eastern redcedar } \\
\text { Other softwoods }\end{array}$ & $\begin{array}{r}8,045 \\
-- \\
-- \\
8,456 \\
-- \\
49,236 \\
13,949 \\
14,013 \\
\end{array}$ & $\begin{array}{l}-- \\
-- \\
-- \\
-- \\
-- \\
-- \\
-- \\
-- \\
--\end{array}$ & $\begin{array}{r}7,810 \\
3,497 \\
38,195 \\
36,149 \\
56,253 \\
58,668 \\
347,361 \\
205,277 \\
13,101 \\
\end{array}$ & $\begin{array}{r}3,378 \\
-- \\
-- \\
-- \\
29,992 \\
-- \\
-- \\
-- \\
-- \\
\end{array}$ & $\begin{array}{r}-- \\
-- \\
2,871 \\
1,199 \\
95,739 \\
-- \\
-- \\
268,256 \\
8,034\end{array}$ & $\begin{array}{r}37,639 \\
-- \\
53,474 \\
21,111 \\
19,803 \\
-- \\
-- \\
367,127 \\
29,221 \\
\end{array}$ & $\begin{array}{l}-- \\
-- \\
-- \\
-- \\
-- \\
-- \\
-- \\
-- \\
--\end{array}$ \\
\hline Total & 93,699 & -- & 766,311 & 33,370 & 376,099 & 528,375 & -- \\
\hline $\begin{array}{l}\text { Hardwoods } \\
\text { Select white oak } \\
\text { Other white oak } \\
\text { Select red oak } \\
\text { other red oak } \\
\text { Select hickory } \\
\text { Other hickory } \\
\text { 8asswood } \\
\text { Beech } \\
\text { Hard maple } \\
\text { Soft maple } \\
\text { Elm } \\
\text { Ash } \\
\text { Sycamore } \\
\text { Cottonwood } \\
\text { Willow } \\
\text { Hackberry } \\
\text { Aspen } \\
\text { Birch } \\
\text { Sweetgum } \\
\text { Tupelo } \\
\text { Black cherry } \\
\text { Black walnut } \\
\text { Butternut } \\
\text { Yellow-poplar } \\
\text { Persimmon } \\
\text { Sassafras } \\
\text { Other hardwoods } \\
\text { Noncommercial spp. }\end{array}$ & $\begin{array}{r}187,025 \\
18,545 \\
80,506 \\
397,516 \\
150,969 \\
21,229 \\
-- \\
48,496 \\
45,260 \\
471,390 \\
69,050 \\
149,976 \\
204,808 \\
-- \\
-- \\
222 \\
22,945 \\
54,888 \\
1,218,716 \\
219,916 \\
23,076 \\
35,453 \\
-- \\
197,348 \\
35,040 \\
133,984 \\
101,728 \\
37,046 \\
\end{array}$ & $\begin{array}{r}457,678 \\
35,181 \\
16,108 \\
1,156,237 \\
75,260 \\
49,114 \\
11,381 \\
-- \\
19,936 \\
178,771 \\
123,582 \\
142,593 \\
4,046 \\
24,607 \\
-- \\
1,135 \\
319 \\
-- \\
110,315 \\
89,822 \\
79,723 \\
8,537 \\
-- \\
-- \\
-- \\
43,038 \\
10,551 \\
3,829 \\
\end{array}$ & $\begin{array}{r}2,026,460 \\
16,822 \\
722,141 \\
2,389,821 \\
1,400,445 \\
919,960 \\
540,752 \\
371,225 \\
1,784,790 \\
10,170,578 \\
5,134,477 \\
5,280,579 \\
5,489,062 \\
3,996,921 \\
1,160,043 \\
1,812,925 \\
107,753 \\
577,204 \\
973,885 \\
346,348 \\
1,159,717 \\
1,778,479 \\
45,885 \\
1,703,767 \\
135,546 \\
1,108,347 \\
3,884,441 \\
1,127,593 \\
\end{array}$ & $\begin{array}{r}-- \\
-- \\
2,285 \\
8,143 \\
-- \\
-- \\
-- \\
-- \\
-- \\
167,741 \\
-- \\
7,338 \\
72,633 \\
951,986 \\
29,128 \\
-- \\
-- \\
-- \\
-- \\
-- \\
6,158 \\
-- \\
-- \\
5,342 \\
-- \\
4,441 \\
30,392 \\
1,707 \\
\end{array}$ & $\begin{array}{r}4,216,552 \\
413,413 \\
3,959,294 \\
2,261,600 \\
3,015,172 \\
3,503,239 \\
1,877,838 \\
9,434,995 \\
20,996,312 \\
3,013,951 \\
3,571,964 \\
3,661,859 \\
1,942,124 \\
169,541 \\
54,222 \\
666,920 \\
216,143 \\
2,371 \\
457,616 \\
756,907 \\
1,985,131 \\
1,582,665 \\
159,426 \\
4,514,556 \\
234,993 \\
1,906,083 \\
2,279,344 \\
1,382,344\end{array}$ & $\begin{array}{r}2,478,646 \\
104,383 \\
1,953,266 \\
1,626,445 \\
1,219,861 \\
2,105,940 \\
325,094 \\
1,141,274 \\
3,752,137 \\
1,111,261 \\
2,794,413 \\
7,060,407 \\
2,013,693 \\
167,806 \\
57,775 \\
607,177 \\
402,384 \\
24,617 \\
348,776 \\
309,914 \\
2,174,503 \\
2,850,487 \\
7,314 \\
8,909,059 \\
55,191 \\
1,340,953 \\
1,483,760 \\
698,534 \\
\end{array}$ & $\begin{array}{r}150,416 \\
-- \\
-- \\
102,260 \\
35,790 \\
-- \\
-- \\
-- \\
-- \\
84,893 \\
27,822 \\
71,291 \\
73,194 \\
56,704 \\
18,125 \\
9,698 \\
-- \\
-- \\
-- \\
-- \\
39,050 \\
-- \\
-- \\
1,081 \\
-- \\
-- \\
225,837 \\
267,295 \\
\end{array}$ \\
\hline Total & $3,925,132$ & $2,541,763$ & $56,165,966$ & $1,287,294$ & $78,236,575$ & $47,125,070$ & $1,163,456$ \\
\hline All species & $4,018,831$ & $2,541,763$ & $56,932,277$ & $1,320,664$ & $78,612,674$ & $47,653,445$ & $1,163,456$ \\
\hline
\end{tabular}


Table 87.--All live tree biomass in timberland by species group and tree biomass component, Indiana, 1986

(1n green tons)

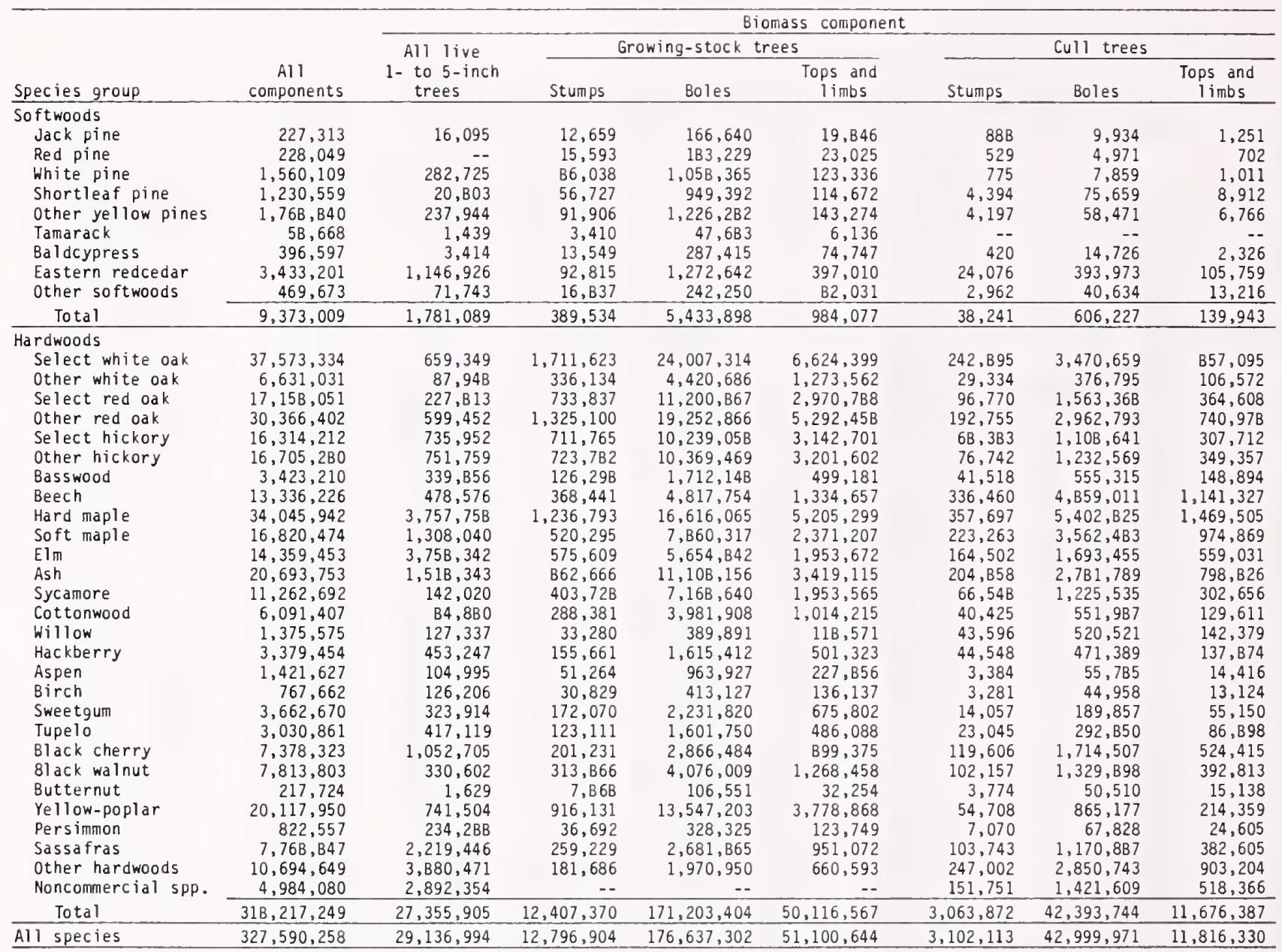


Table 88.--All live tree biomass on timberland by species group and tree biomass component, Indiana, 1986

(In thousand cubic feet)

\begin{tabular}{|c|c|c|c|c|c|c|c|c|}
\hline \multirow[b]{3}{*}{ Species group } & \multirow[b]{3}{*}{$\begin{array}{c}\text { All } \\
\text { components }\end{array}$} & \multicolumn{7}{|c|}{ Biomass component } \\
\hline & & \multirow{2}{*}{$\begin{array}{l}\text { All live } \\
1 \text { - to } 5 \text {-inch } \\
\text { trees }\end{array}$} & \multicolumn{3}{|c|}{ Growing-stock trees } & \multicolumn{3}{|c|}{ Cull trees } \\
\hline & & & Stumps & Boles & $\begin{array}{c}\text { Tops and } \\
1 \text { imbs }\end{array}$ & Stumps & Boles & $\begin{array}{l}\text { Tops and } \\
\text { limbs }\end{array}$ \\
\hline \multicolumn{9}{|l|}{ So ftwoods } \\
\hline Jack pine & 9,889 & 702 & 554 & 7,247 & 861 & 38 & 432 & 55 \\
\hline Red pine & 9,864 & - & 679 & 7,923 & 993 & 23 & 215 & 31 \\
\hline White pine & 69,167 & 12,653 & 3,809 & 46,826 & 5,452 & 34 & 348 & 45 \\
\hline Shortleaf pine & 44,242 & 763 & 2,040 & 34,118 & 4,123 & 157 & 2,720 & 321 \\
\hline other yellow pines & 78,627 & 10,578 & 4,094 & 54,503 & 6,366 & 187 & 2,598 & 301 \\
\hline Tamarack & 2,233 & & 130 & 1,813 & 233 & - & & \\
\hline Baldcypress & 13,973 & 120 & 478 & 10,128 & 2,631 & & 519 & \\
\hline Eastern redcedar & 161,076 & 53,933 & 4,351 & 59,629 & 18,613 & 1,134 & 18,461 & 4,955 \\
\hline Other softwoods & 21,443 & 3,275 & 764 & 11,059 & 3,750 & 137 & 1,855 & 603 \\
\hline Total & 410,514 & 82,081 & 16,899 & 233,246 & 43,022 & 1,725 & 27,148 & 6,393 \\
\hline \multicolumn{9}{|l|}{ Hardwoods } \\
\hline Select white oak & $1,293,756$ & 22,710 & 58,971 & 826,628 & 228,077 & 8,368 & 119,490 & 29,512 \\
\hline other white oak & 228,337 & 3,028 & 11,581 & 152,228 & 43,847 & 1,008 & 12,974 & 3,671 \\
\hline Select red oak & 533,260 & 7,082 & 22,811 & 348,109 & 92,334 & 2,998 & 48,589 & 11,337 \\
\hline other red oak & 942,554 & 18,643 & 41,118 & 597,599 & 164,256 & 5,981 & 91,956 & 23,001 \\
\hline Select hickory & 514,483 & 23,259 & 22,447 & 322,866 & 99,100 & 2,151 & 34,957 & 9,703 \\
\hline other hickory & 566,294 & 25,491 & 24,529 & 351,514 & 108,531 & 2,602 & 41,781 & 11,846 \\
\hline Basswood & 162,421 & 16,319 & 5,982 & 81,135 & 23,649 & 1,968 & 26,314 & 7,054 \\
\hline Beech & 451,602 & 16,209 & 12,495 & 163,126 & 45,193 & 11,403 & 164,532 & 38,644 \\
\hline Hard maple & $1,084,652$ & 119,846 & 39,420 & 529,285 & 165,794 & 11,398 & 172,098 & 46,811 \\
\hline So ft maple & 608,015 & 47,206 & 18,818 & 284,162 & 85,721 & 8,078 & 128,780 & 35,250 \\
\hline Elm & 527,535 & 142,416 & 20,928 & 205,429 & 70,961 & 5,974 & 61,516 & 20,311 \\
\hline Ash & 809,695 & 60,873 & 33,686 & 433,773 & 133,525 & 8,012 & 108,634 & 31,192 \\
\hline Sycamore & 389,361 & 4,921 & 13,933 & 247,825 & 67,548 & 2,299 & 42,370 & 10,465 \\
\hline Cottonwood & 238,089 & 3,325 & 11,289 & 155,616 & 39,636 & 1,585 & 21,574 & 5,064 \\
\hline Willow & 57,609 & 5,372 & 1,395 & 16,315 & 4,961 & 1,826 & 21,784 & 5,956 \\
\hline Hackberry & 129,661 & 17,393 & 5,981 & 61,968 & 19,237 & 1,708 & 18,084 & 5,290 \\
\hline Aspen & 56,711 & 4,236 & 2,043 & 38,415 & 9,082 & 135 & 2,226 & 574 \\
\hline 8irch & 28,011 & 4,621 & 1,129 & 15,086 & 4,969 & 119 & 1,616 & 471 \\
\hline Sweetgum & 121,827 & 10,767 & 5,733 & 74,235 & 22,475 & 470 & 6,313 & 1,834 \\
\hline Tupelo & 104,498 & 14,371 & 4,243 & 55,232 & 16,762 & 800 & 10,095 & 2,995 \\
\hline Black cherry & 299,951 & 42,723 & 8,185 & 116,561 & 36,573 & 4,864 & 69,723 & 21,322 \\
\hline Black walnut & 292,133 & 12,957 & 11,713 & 152,069 & 47,315 & 3,808 & 49,616 & 14,655 \\
\hline Butternut & 9,723 & 73 & 353 & 4,757 & 1,441 & 168 & 2,256 & 675 \\
\hline Yellow-poplar & 771,842 & 28,458 & 35,158 & 519,741 & 144,968 & 2,089 & 33,201 & 8,227 \\
\hline Persimmon & 35,658 & 10,158 & 1,595 & 14,234 & 5,358 & 305 & 2,941 & 1,067 \\
\hline Sassafras & 336,779 & 96,179 & 11,238 & 116,275 & 41,237 & 4,510 & 50,755 & 16,585 \\
\hline other hardwoods & 463,656 & 168,216 & 7,884 & 85,457 & 28,641 & 10,710 & 123,597 & 39,151 \\
\hline \multirow{2}{*}{$\begin{array}{l}\text { Noncommercial spp. } \\
\text { Total }\end{array}$} & 199,403 & 115,731 & -- & & -- & 6,076 & 56,866 & 20,730 \\
\hline & $11,257,516$ & $1,042,583$ & 434,658 & $5,969,640$ & $1,751,191$ & 111,413 & $1,524,638$ & 423,393 \\
\hline All species & $11,668,030$ & $1,124,664$ & 451,557 & $6,202,886$ & $1,794,213$ & 113,138 & $1,551,786$ & 429,786 \\
\hline
\end{tabular}


Table 89.--All live shrubl/ biomass yields on timberland by shrub species group and forest type, Indiana, 1986

(In pounds per acre green weight)

\begin{tabular}{|c|c|c|c|c|c|c|c|}
\hline \multirow[b]{2}{*}{ Shrub species group } & \multirow[b]{2}{*}{ Pine } & \multicolumn{6}{|c|}{ Forest type } \\
\hline & & $\begin{array}{l}\text { Oak- } \\
\text { pine }\end{array}$ & $\begin{array}{c}\text { Oak- } \\
\text { hickory }\end{array}$ & $\begin{array}{l}\text { Oak - } \\
\text { gum }\end{array}$ & $\begin{array}{l}\text { Elm-ash- } \\
\text { soft } \\
\text { maple }\end{array}$ & $\begin{array}{l}\text { Maple- } \\
\text { beech }\end{array}$ & $\begin{array}{l}\text { Cherry-ash- } \\
\text { yellow } \\
\text { poplar }\end{array}$ \\
\hline \multicolumn{8}{|l|}{ Tall shrubs } \\
\hline White pine & 171 & -- & $-\infty$ & -- & -- & -- & -- \\
\hline Virginia pine & - & -- & 8 & -- & -- & -- & -- \\
\hline Eastern redcedar & -- & 165 & 8 & 114 & 18 & 15 & 21 \\
\hline Select white oak & -- & 6 & 31 & -- & 24 & 12 & 44 \\
\hline other white oak & -- & -- & 1 & -- & - & 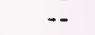 & -- \\
\hline Select red oak & 113 & 5 & 17 & 5 & 1 & 4 & -- \\
\hline other red oak & 77 & 29 & 20 & 295 & 15 & 4 & 6 \\
\hline Select hickory & -- & - & 32 & 7 & 18 & 1 & 17 \\
\hline other hickory & 34 & 8 & 9 & 11 & 22 & 39 & 26 \\
\hline Basswood & -- & 1 & 7 & - & - & 56 & - \\
\hline Beech & 116 & 84 & 92 & 14 & 3 & 87 & 41 \\
\hline Hard maple & 170 & 37 & 255 & 11 & 66 & 411 & 181 \\
\hline Soft maple & 43 & 132 & 56 & 1,089 & 47 & 9 & 3 \\
\hline Elm & 145 & 11 & 225 & 10 & 112 & 178 & 165 \\
\hline Black ash & - & $\ldots$ & 4 & $\ldots$ & - & 23 & 3 \\
\hline White \& green ash & 197 & 90 & 178 & 156 & 91 & 90 & 391 \\
\hline Sycamore & -- & 4 & -- & - & - & 18 & - \\
\hline Willow & -- & -- & -- & -- & 25 & 1 & -- \\
\hline Hackberry & 34 & 3 & 44 & -- & 22 & 9 & 43 \\
\hline Sweetgum & -- & 1 & - & 248 & 26 & -- & -- \\
\hline Tupelo & 64 & -- & 48 & 44 & 6 & 6 & 24 \\
\hline Black cherry & 585 & 33 & 80 & 151 & 29 & 103 & 41 \\
\hline Black walnut & - & -- & 7 & -- & 18 & 1 & 1 \\
\hline Yellow-poplar & 232 & 3 & 5 & 66 & 15 & 6 & 9 \\
\hline other hardwoods & 583 & 528 & 267 & 482 & 340 & 307 & 544 \\
\hline American hornbeam & 3 & -- & 78 & -- & 2 & 119 & 84 \\
\hline Eastern redbud & -- & 58 & 37 & -- & 34 & 36 & 30 \\
\hline Osage-orange & -- & - & 10 & -- & 3 & -- & - \\
\hline Apple & -- & 1 & 25 & -- & 1 & 6 & -- \\
\hline Eastern hophornbeam & 1 & 2 & 102 & -- & 13 & 65 & 25 \\
\hline Chokecherry & 6 & -- & 4 & -- & 8 & 12 & 2 \\
\hline Dogwood & 1 & 21 & 52 & 13 & 154 & 13 & 70 \\
\hline Pawpaw & -- & 7 & 33 & 841 & 9 & 110 & 49 \\
\hline Witch hazel & -- & -- & 1 & -- & 357 & -- & 29 \\
\hline Juneberry & -- & -- & 11 & -- & -- & - & 43 \\
\hline Hazel & -- & -- & 1 & -- & 3 & 9 & 19 \\
\hline Prickly ash & -- & - & 14 & - & 23 & 93 & 1,456 \\
\hline Alder buckthorn & -- & -- & 2 & -- & - & 1 & 1,700 \\
\hline Viburnum & -- & -- & 9 & -- & 6 & 2 & 3 \\
\hline Elder & -- & -- & 1 & $-\rightarrow$ & 2 & 76 & 2 \\
\hline Sumac & -- & 386 & 43 & 1,204 & 61 & 7 & 148 \\
\hline Shrubby willows & - & 300 & 40 & 1,204 & 33 & -- & $\begin{array}{l}140 \\
379\end{array}$ \\
\hline Misc. tall shrubs & 1 & 1 & 214 & 112 & 743 & 77 & 68 \\
\hline Total tall shrubs & 2,576 & 1,616 & 2,031 & 4,873 & 2.350 & 2,006 & 3,967 \\
\hline \multicolumn{8}{|l|}{ Low shrubs } \\
\hline Virginia creeper & 243 & 38 & 134 & 23 & 91 & 91 & 43 \\
\hline Gooseberry-current & 4 & 4 & 4 & - & 8 & 13 & g \\
\hline Raspberry-blackberry & 58 & 5 & 75 & 634 & 82 & 44 & 145 \\
\hline Rose & 4 & 7 & 7 & 6 & 27 & 12 & 12 \\
\hline Bilberry-blueberry & -- & 3 & 10 & $\ldots$ & $-\infty$ & - & 3 \\
\hline Honeysuckle & 132 & 140 & 16 & 89 & 24 & 36 & 86 \\
\hline Snowberry & $\ldots$ & - & - & 9 & -- & -- & -. \\
\hline Poison ivy & 164 & 82 & 148 & 169 & 115 & 54 & 109 \\
\hline Greenbriar & 25 & 11 & 54 & 11 & 19 & 8 & 22 \\
\hline Grape & 4 & 6 & 12 & 6 & 7 & $\mathrm{~g}$ & 18 \\
\hline Misc. low shrubs & 9 & 5 & 23 & 2 & 38 & 11 & 47 \\
\hline Total low shrubs & 643 & 301 & 483 & 949 & 411 & 278 & 494 \\
\hline All shrub species & 3,219 & 1,917 & 2,514 & 5,822 & 2,761 & 2.284 & 4,461 \\
\hline Number of plots $2 /$ & 14 & 15 & 148 & 7 & 96 & 130 & 85 \\
\hline
\end{tabular}

1/ Trees under 1.0 inch d.b.h. are al so included.

2/ Number of plots by forest type from which average yields were derived. 
Table 90.--Sampling errors for Forest Survey Unit and county totals of volume, net annual growth, average annual removals, and area of timberland

(In percent)

\begin{tabular}{|c|c|c|c|c|c|c|c|}
\hline \multirow[b]{2}{*}{ Unit and county } & \multirow[b]{2}{*}{ Area } & \multicolumn{3}{|c|}{ Growing stock } & \multicolumn{3}{|c|}{ Sawt imber } \\
\hline & & Vol ume & Growth & Removals $2 /$ & Vol ume & Growth & Removals \\
\hline $\begin{array}{l}\text { Lower Wabash Unit } \\
\text { Clay } \\
\text { Daviess } \\
\text { Gibson } \\
\text { Greene } \\
\text { Knox } \\
\text { Martin } \\
\text { Parke } \\
\text { Pike } \\
\text { Posey } \\
\text { Putnam } \\
\text { Sullivan } \\
\text { Vanderburgh } \\
\text { Vermillion } \\
\text { Vigo } \\
\text { Total } \\
\end{array}$ & $\begin{array}{r}11.11 \\
11.46 \\
11.41 \\
7.20 \\
13.32 \\
6.52 \\
7.90 \\
8.07 \\
11.01 \\
8.45 \\
9.19 \\
14.87 \\
12.55 \\
10.37 \\
2.52 \\
\end{array}$ & $\begin{array}{r}15.76 \\
16.21 \\
16.60 \\
10.38 \\
19.81 \\
8.60 \\
10.88 \\
11.29 \\
14.91 \\
11.69 \\
12.87 \\
21.47 \\
18.01 \\
15.15 \\
3.53 \\
\end{array}$ & $\begin{array}{r}21.69 \\
22.69 \\
22.52 \\
13.99 \\
27.71 \\
12.85 \\
14.72 \\
15.39 \\
21.30 \\
16.75 \\
17.66 \\
29.17 \\
25.02 \\
20.66 \\
4.92 \\
\end{array}$ & $\begin{array}{l}55.98 \\
54.71 \\
50.38 \\
41.57 \\
57.93 \\
31.34 \\
37.13 \\
44.35 \\
40.47 \\
38.04 \\
46.68 \\
72.12 \\
67.11 \\
56.46 \\
12.28 \\
\end{array}$ & $\begin{array}{r}18.42 \\
18.94 \\
19.66 \\
12.29 \\
23.30 \\
9.83 \\
12.85 \\
13.33 \\
17.26 \\
13.52 \\
15.13 \\
25.36 \\
21.14 \\
17.96 \\
4.13 \\
\end{array}$ & $\begin{array}{r}12.59 \\
14.10 \\
14.34 \\
8.93 \\
15.90 \\
7.40 \\
9.37 \\
9.84 \\
12.58 \\
8.96 \\
11.21 \\
17.48 \\
15.20 \\
13.07 \\
2.98 \\
\end{array}$ & $\begin{array}{l}57.45 \\
55.87 \\
51.42 \\
42.77 \\
59.76 \\
31.42 \\
37.99 \\
45.38 \\
41.16 \\
38.26 \\
47.84 \\
77.48 \\
68.94 \\
58.71 \\
12.54 \\
\end{array}$ \\
\hline $\begin{array}{l}\text { Knobs Unit } \\
\text { Brown } \\
\text { Clark } \\
\text { Crawford } \\
\text { Dubois } \\
\text { Floyd } \\
\text { Harrison } \\
\text { Jackson } \\
\text { Lawrence } \\
\text { Monroe } \\
\text { Morgan } \\
\text { Orange } \\
\text { Owen } \\
\text { Perry } \\
\text { Scott } \\
\text { Spencer } \\
\text { Warrick } \\
\text { Washington } \\
\text { Total } \\
\end{array}$ & $\begin{array}{l}3.02 \\
3.76 \\
3.17 \\
3.59 \\
5.90 \\
3.01 \\
3.15 \\
3.09 \\
3.20 \\
3.72 \\
3.05 \\
3.34 \\
2.80 \\
5.28 \\
4.38 \\
3.84 \\
3.17 \\
0.83 \\
\end{array}$ & $\begin{array}{r}6.85 \\
8.91 \\
7.54 \\
8.49 \\
13.73 \\
7.13 \\
7.49 \\
7.03 \\
7.36 \\
9.04 \\
7.11 \\
7.98 \\
6.47 \\
12.67 \\
10.67 \\
9.66 \\
7.48 \\
1.95 \\
\end{array}$ & $\begin{array}{l}11.65 \\
14.77 \\
12.56 \\
14.26 \\
23.10 \\
11.64 \\
12.13 \\
12.03 \\
12.30 \\
14.76 \\
11.90 \\
13.24 \\
10.95 \\
20.69 \\
17.40 \\
15.07 \\
12.44 \\
3.24 \\
\end{array}$ & $\begin{array}{l}23.60 \\
36.58 \\
26.30 \\
34.83 \\
46.32 \\
30.75 \\
29.32 \\
26.75 \\
19.94 \\
36.23 \\
28.43 \\
32.59 \\
23.70 \\
50.61 \\
42.81 \\
40.38 \\
30.35 \\
7.26 \\
\end{array}$ & $\begin{array}{r}8.35 \\
10.88 \\
9.25 \\
10.29 \\
16.64 \\
8.81 \\
9.31 \\
8.51 \\
8.98 \\
11.14 \\
8.76 \\
9.71 \\
8.00 \\
15.57 \\
13.08 \\
12.09 \\
9.09 \\
2.39 \\
\end{array}$ & $\begin{array}{r}19.72 \\
25.57 \\
21.65 \\
24.18 \\
36.57 \\
20.99 \\
22.07 \\
20.09 \\
21.47 \\
26.64 \\
20.45 \\
21.69 \\
18.46 \\
37.26 \\
31.55 \\
28.82 \\
19.83 \\
5.58 \\
\end{array}$ & $\begin{array}{r}25.25 \\
38.87 \\
29.18 \\
36.53 \\
48.56 \\
32.93 \\
32.07 \\
29.03 \\
21.98 \\
39.30 \\
31.46 \\
34.22 \\
26.28 \\
53.45 \\
45.81 \\
43.42 \\
32.04 \\
7.87 \\
\end{array}$ \\
\hline $\begin{array}{l}\text { Upland Flats Unit } \\
\text { Dearborn } \\
\text { Fayette } \\
\text { Frankl in } \\
\text { Jefferson } \\
\text { Jennings } \\
\text { Ohio } \\
\text { Ripley } \\
\text { Switzerland } \\
\text { Union } \\
\text { Total }\end{array}$ & $\begin{array}{r}8.86 \\
14.62 \\
9.43 \\
9.56 \\
9.04 \\
16.02 \\
9.72 \\
9.76 \\
18.29 \\
3.54\end{array}$ & $\begin{array}{l}13.38 \\
24.73 \\
15.86 \\
13.47 \\
13.68 \\
24.76 \\
14.27 \\
13.10 \\
31.26 \\
5.32\end{array}$ & $\begin{array}{l}18.54 \\
32.24 \\
20.44 \\
19.66 \\
18.85 \\
33.84 \\
20.45 \\
19.81 \\
41.02 \\
7.45\end{array}$ & $\begin{array}{l}55.22 \text {. } \\
{ }^{5} .80 \\
52.80 \\
54.85 \\
\star \\
53.03 \\
47.80 \\
{ }^{\star 0} \\
21.06\end{array}$ & $\begin{array}{l}16.95 \\
32.61 \\
20.80 \\
16.83 \\
17.32 \\
31.54 \\
17.83 \\
15.92 \\
41.02 \\
6.71 \\
\end{array}$ & $\begin{array}{l}28.63 \\
51.41 \\
32.71 \\
26.89 \\
28.96 \\
52.83 \\
28.43 \\
27.36 \\
65.45 \\
11.01\end{array}$ & $\begin{array}{c}54.87 \\
\star \\
71.39 \\
52.41 \\
54.95 \\
\star \\
53.82 \\
47.20 \\
\star \\
21.13\end{array}$ \\
\hline
\end{tabular}

$1 /$ * indicates a sampling error over 99.00 percent.

(Table 90 continued on next page)

2/Error figures are for average annual removals. 
(Table 90 continued)

\begin{tabular}{|c|c|c|c|c|c|c|c|}
\hline \multirow[b]{2}{*}{ Unit and county } & \multirow[b]{2}{*}{ Area } & \multicolumn{3}{|c|}{ Growing stock } & \multicolumn{3}{|c|}{ Sawtimber } \\
\hline & & Vol ume & Growth & Removals $2 /$ & Vol ume & Growth & Removals \\
\hline \multicolumn{8}{|l|}{ Northern Unit } \\
\hline Adams & 21.78 & 33.52 & 88.35 & 97.99 & 37.45 & * & $\star$ \\
\hline Allen & 14.98 & 26.15 & 57.03 & 64.17 & 29.89 & 93.49 & 66.06 \\
\hline 8artholomew & 12.22 & 18.57 & 53.20 & 53.96 & 20.59 & 80.92 & 54.59 \\
\hline Benton & 66.77 & 97.55 & * & $\star$ & * & * & \# \\
\hline 81 ackford & 26.67 & 39.18 & * & * & 43.07 & * & $\star$ \\
\hline 8oone & 20.97 & 32.55 & 84.96 & 85.78 & 36.04 & * & 87.23 \\
\hline Carroll & 19.06 & 29.60 & 75.87 & 83.35 & 33.03 & * & 84.91 \\
\hline Cass & 16.62 & 26.55 & 68.63 & 87.75 & 30.20 & * & 91.19 \\
\hline Clinton & 26.96 & 40.31 & * & $\star$ & 44.26 & * & ฝ \\
\hline Decatur & 16.66 & 24.55 & 71.15 & 67.00 & 27.04 & $\star$ & 67.94 \\
\hline De Kalb & 15.65 & 25.07 & 64.78 & 88.02 & 28.67 & * & 91.80 \\
\hline Del aware & 20.57 & 33.41 & 80.82 & 94.08 & 38.00 & $\star$ & 98.27 \\
\hline El khart & 15.16 & 24.66 & 56.11 & 71.24 & 27.96 & 93.44 & 72.97 \\
\hline Founta in & 13.52 & 21.08 & 56.43 & 71.98 & 23.89 & 84.76 & 74.10 \\
\hline Fulton & 18.11 & 28.73 & 76.03 & 90.00 & 32.47 & * & 92.55 \\
\hline Grant & 19.17 & 28.43 & 78.90 & 79.05 & 31.63 & * & 81.07 \\
\hline Hamilton & 18.29 & 29.25 & 67.22 & 87.21 & 33.34 & * & 90.54 \\
\hline Hancock & 23.81 & 37.99 & 95.59 & $\star$ & 43.44 & * & * \\
\hline Hendricks & 19.44 & 30.99 & 83.79 & 87.75 & 34.54 & * & 89.52 \\
\hline Henry & 18.02 & 28.54 & 74.28 & 83.82 & 32.13 & * & 86.54 \\
\hline Howard & 27.88 & 47.91 & $\star$ & * & 54.14 & * & * \\
\hline Huntington & 17.09 & 28.19 & 71.11 & 88.43 & 32.19 & $\star$ & 92.00 \\
\hline Jasper & 15.74 & 25.45 & 61.10 & 73.21 & 28.73 & 99.00 & 75.01 \\
\hline Jay & 16.69 & 24.47 & 75.50 & 69.90 & 26.86 & $\star$ & 70.46 \\
\hline Johnson & 18.24 & 27.59 & 80.06 & 78.46 & 30.41 & * & 79.34 \\
\hline Kosciusko & 14.24 & 20.97 & 63.66 & 59.82 & 23.07 & 96.49 & 60.40 \\
\hline La Grange & 13.65 & 23.00 & 58.10 & 76.41 & 26.30 & 88.49 & 79.60 \\
\hline Lake & 19.38 & 30.09 & 78.14 & 76.14 & 33.95 & * & 80.07 \\
\hline La Porte & 12.71 & 20.43 & 48.50 & 57.89 & 23.04 & 79.20 & 59.22 \\
\hline Madison & 22.68 & 34.06 & 97.00 & 89.00 & 37.80 & * & 91.83 \\
\hline Marion & $\star$ & $\star$ & $\star$ & 72.98 & $\star$ & $\star$ & \# \\
\hline Marshall & 14.69 & 23.67 & 64.29 & 75.52 & 26.67 & 98.85 & 77.16 \\
\hline Miami & 16.23 & 23.82 & 70.00 & 70.04 & 26.34 & $*$ & 70.94 \\
\hline Montgomery & 16.69 & 26.65 & 63.63 & 81.44 & 30.51 & 97.74 & 85.08 \\
\hline Newton & 19.33 & 28.53 & 78.14 & 88.30 & 32.03 & * & 91.16 \\
\hline Noble & 14.39 & 23.45 & 57.58 & 71.98 & 26.73 & 88.83 & 74.37 \\
\hline Porter & 14.78 & 24.14 & 56.83 & 72.28 & 27.66 & 87.19 & 76.08 \\
\hline Pulaski & 15.74 & $\begin{array}{l}24.14 \\
25.00\end{array}$ & 62.24 & 77.98 & 28.32 & 97.83 & 79.98 \\
\hline Randolph & 18.42 & 27.05 & $\begin{array}{l}02.24 \\
78.78\end{array}$ & 71.17 & 29.72 & * & 72.42 \\
\hline Rush & 23.22 & 40.67 & 90.34 & 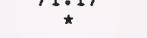 & 46.38 & * & ${ }^{4}$ \\
\hline St. Joseph & 17.24 & 28.34 & 64.29 & 76.68 & 32.35 & * & 79.87 \\
\hline Shelby & 23.13 & 34.10 & $\star$ & 99.34 & 37.72 & * & $\star$ \\
\hline Starke & 15.77 & 24.76 & 62.53 & 75.96 & 27.85 & 98.62 & 77.28 \\
\hline Steuben & 14.57 & 23.96 & 58.00 & 74.42 & 27.37 & 89.87 & 76.84 \\
\hline Tippecanoe & 17.20 & 27.92 & 66.57 & 86.03 & 32.05 & $\star$ & 90.38 \\
\hline Tipton & 37.72 & 66.21 & $\star$ & * & 75.43 & * & $\star$ \\
\hline Wabash & 16.69 & 27.28 & 65.69 & 85.15 & 31.20 & * & 89.39 \\
\hline Warren & 16.76 & 26.11 & 68.91 & 77.41 & 29.20 & * & 78.65 \\
\hline Wayne & 14.48 & 23.35 & 61.74 & 72.36 & 26.45 & 93.45 & 74.49 \\
\hline Wells & 20.32 & 32.12 & 79.20 & 96.87 & 36.61 & * & $\star$ \\
\hline Whi te & 22.68 & 38.52 & 89.81 & * & 44.42 & * & * \\
\hline Whitley & 18.06 & 29.26 & 76.57 & 98.56 & 33.29 & * & * \\
\hline Total & 2.44 & 3.88 & 9.96 & 11.23 & 4.37 & 15.47 & 11.56 \\
\hline Al I counties & 1.00 & 1.57 & 3.42 & 5.40 & 1.86 & 5.47 & 5.68 \\
\hline
\end{tabular}

1/ * indicates a sampling error over 99.00 percent.

2/ Error figures are for average annual removals. 


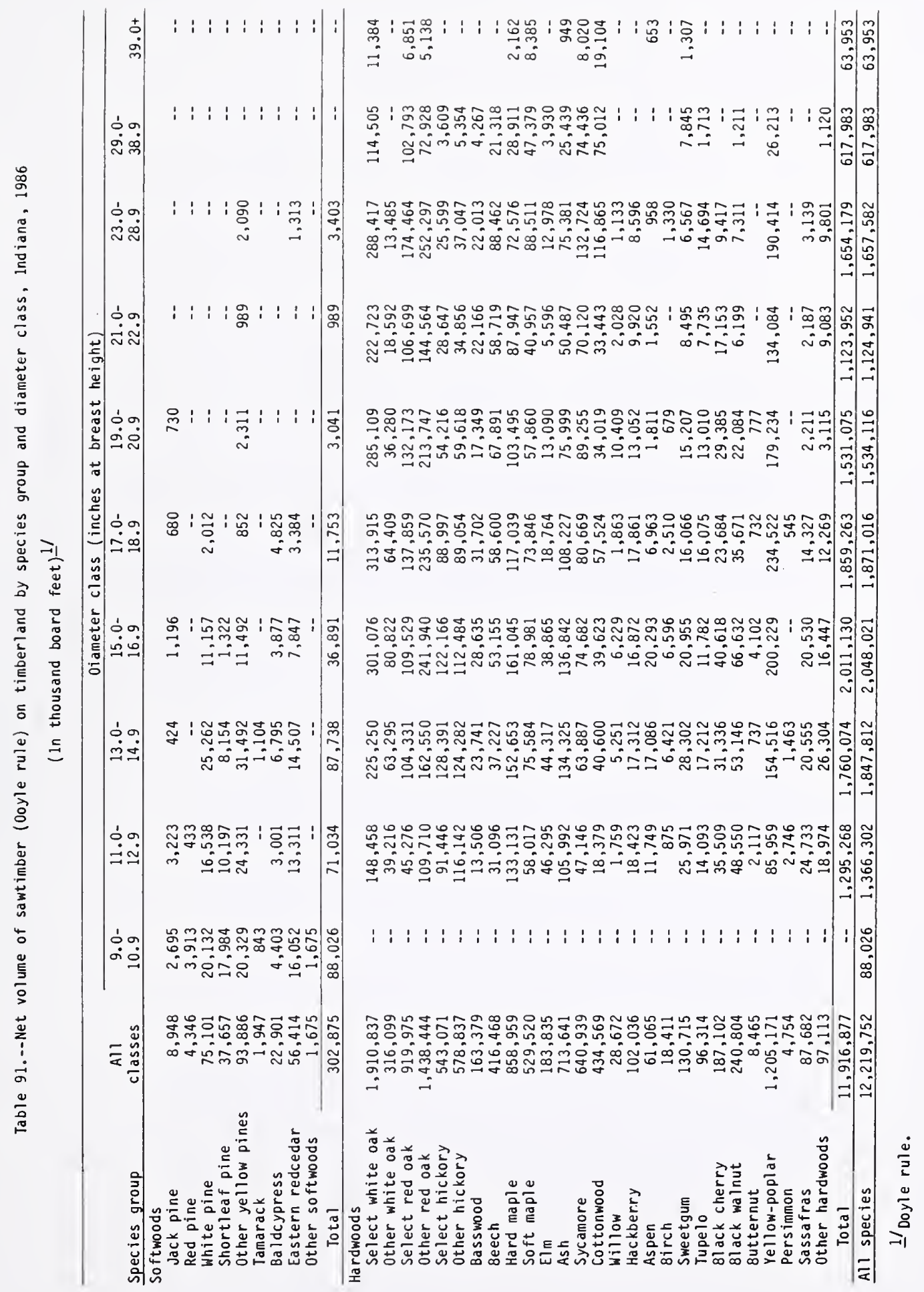



Smith, W. Brad; Golitz, Mark F.

1988. Indiana forest statistics, 1986. Resour. Bull. NC-108. St. Paul, MN: U.S. Department of Agriculture, Forest Service, North Central Forest Experiment Station. 139 p.

The third inventory of Indiana's timber resource shows that timberland area in Indiana climbed from 3.9 to 4.3 million acres between 1967 and 1986, an increase of more than 10 percent. During the same period growing-stock volume increased 43 percent. Highlights and statistics are presented on area, volume, growth, mortality, and removals.

KEY WORDS: Area, volume, growth, mortality, removals. 
Our job at the North Central Forest Experiment Station is discovering and creating new knowledge and technology in the field of natural resources and conveying this information to the people who can use it. As a new generation of forests emerges in our region, managers are confronted with two unique challenges: (1) Dealing with the great diversity in composition, quality, and ownership of the forests, and (2) Reconciling the conflicting demands of the people who use them. Helping the forest manager meet these challenges while protecting the environment is what research at North Central is all about.

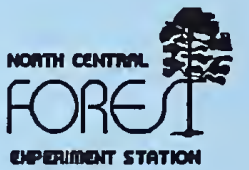

